\title{
Non-Radiative Processes and Vibrational Pumping in SURFACE-ENHANCED RAMAN SCATTERING
}

\author{
by \\ Christopher Galloway \\ A thesis \\ Doctor of Philosophy \\ in Physics
}

submitted to the Victoria University of Wellington in fulfilment of the requirements for the degree of

Victoria University of Wellington

The MacDiarmid Institute for Advanced Materials and Nanotechnology

2010 


\section{Abstract}

The main focus of this thesis was the physical interpretation of the pumping cross-section. This was achieved by performing a statistical analysis of single molecule vibrational pumping events in which both the SERS and pumping cross-sections could be measured simultaneously. Samples were constructed in which small aggregates of silver colloids were evenly distributed on a dry surface. The sample was then cooled to $77 \mathrm{~K}$ so that the main mechanism for creating a vibrational population was through Stokes scattering. Spatial mappings were then performed which measured how the SERS spectrum varied with position on the sample and the single molecule events were identified. The SERS crosssections were determined from the Stokes intensity while the pumping crosssections were determined from the ratio of the anti-Stokes and Stokes peaks. It was observed that the pumping cross-section was often significantly larger than the SERS cross-section, as much as four orders of magnitude in some cases. Several attempts were made to explain this discrepancy including the possibility of the surface plasmon resonance favouring anti-Stokes scattering, underestimated lifetimes for the vibrational modes, and additional pumping from fluorescence. However, the most likely candidate was non-radiative Stokes scattering by the observed molecule which would increase the vibrational population but would not increase the Stokes intensity.

To estimate the proportion of scattered light that is radiative or non-radiative, single molecule measurements were performed under both surface-enhanced and unmodified conditions. By comparing the fluorescence and Raman intensities under these scenarios, it was possible to estimate the radiative and non-radiative enhancement factors. It was found that the non-radiative SERS cross-section was typically much larger than the radiative cross-section for samples consisting of aggregated silver colloids. The discrepancy between the pumping and SERS cross-section (which is the radiative cross-section) could therefore be explained by non-radiative scattering dominating the creation of the vibrational population, along with an additional contribution due to the plasmon resonance favouring certain vibrational modes. Furthermore, the lifetime of a molecule after it has been excited to the first electronic state was estimated to be as short as $25 \mathrm{fs}$. It would be impossible to measure lifetimes of this order of magnitude in single molecules using time-resolved techniques. Furthermore, to the very best of our knowledge, this is the first time that an experimental determination of the non-radiative SERS cross-section has been made. 


\section{Acknowledgements}

My time as a PhD student in the Raman group at Victoria University has been an extremely enjoyable and relaxing time. This has been mostly due to the support and encouragement I have received from my colleagues and supervisors. The work in this thesis is very much a collaborative effort which I happen to have the honour of presenting. Consequently, there are many people who must be thanked without which I would never have reached this level of academic achievement. First among them is my supervisors Pablo Etchegoin and Eric Le $\mathrm{Ru}$, both of which are extremely patient, knowledgeable and hard-working, yet always approachable. I would also like to thank my colleagues within the Raman group: Evan Blackie, Matthias Meyer, Andrew Preston and Stefan Andreas Meyer, who have made significant contributions to this research over the last three years. The opportunity to work with such a talented group of people has been a great honour.

I am grateful to people within the school of chemical and physical sciences at Victoria University for their continuous support, most notably: Margaret Brown, Ben Ruck, Joe Trodahl, Paul Callahan and John Spencer. I would also like to thank the lecturers that have taught me over the last nine years and have stimulated my interest in science.

In addition, I would like to express my gratitude to the MacDiarmid Institute, the Marsden Fund, and Victoria University of Wellington for their financial support over the last three years, without which I would never have been able to undertake this research.

Finally, I am eternally indebted to my friends and family. Most importantly are my parents, their encouragement, understanding and guidance has been steadfast and I will be forever grateful for their support. I am also thankful to my close friends for keeping me grounded and helping me to unwind, especially over this period of thesis writing. 


\section{Contents}

$\begin{array}{lll}1 & \text { Introduction } & 7\end{array}$

2 The Interaction between Light and Matter 11

2.1 Molecular Transitions . . . . . . . . . . . . . . . . . . . . 11

2.2 Molecular Vibrations . . . . . . . . . . . . . . . . . . . 13

2.3 The Raman Effect . . . . . . . . . . . . . . . . . . . . 15

2.3.1 A Classical Theory of Raman and Rayleigh Scattering . . 16

2.3.2 Limitations of the Classical Theory . . . . . . . . . . . . 19

2.3.3 Stokes and anti-Stokes Scattering . . . . . . . . . . . 20

2.3.4 Types of Raman Scattering . . . . . . . . . . . . . . 22

2.3.5 Resonance Raman Scattering . . . . . . . . . . . . . . . 24

2.4 Absorption and Fluorescence Spectroscopy . . . . . . . . . . . . 26

2.4 .1 Decay Rates . . . . . . . . . . . . . . . . . . . 26

2.4.2 The Fluorescence Spectrum . . . . . . . . . . . . . 27

2.4.3 Interactions with the Triplet State . . . . . . . . . . 29

3 The Surface Enhancement 31

3.1 Surface Plasmon Resonances . . . . . . . . . . . . . . . . . 31

3.1.1 Surface Plasmons . . . . . . . . . . . . . . . . . . . . . 31

3.1.2 Localised Surface Plasmons . . . . . . . . . . . . . . 32

3.1.3 The Effect of the Dielectric Function . . . . . . . . . . . 33

3.2 Raman and Fluorescence Enhancement Factors . . . . . . . . . 35

3.2.1 Surface Enhanced Raman Scattering . . . . . . . . . . 35

3.2.2 Surface Enhanced Fluorescence . . . . . . . . . . . . 37

3.3 Examples of SERS Enhancements _. . . . . . . . . . . 41

3.3.1 The Metallic Sphere . . . . . . . . . . . . . . . . . . 41

3.3.2 The Metallic Dimer . . . . . . . . . . . . . . . . . 44 
3.3.3 Large Colloidal Aggregates . . . . . . . . . . . . . 51

4 Vibrational Pumping $\quad 55$

4.1 Introduction . . . . . . . . . . . . . . . . 55

4.2 The Theory of Vibrational Pumping . . . . . . . . 56

4.2.1 The anti-Stokes and Stokes Intensity . . . . . . . . 56

4.2 .2 Laser Heating . . . . . . . . . . . . . . . . . . . 59

4.2.3 The Temperature Dependence . . . . . . . . . . 61

4.2.4 The Asymmetry Factor and Resonance Effects . . . . . . 63

4.2.5 The Pumping Cross-Section and Spatial Averaging . . . . 65

4.2.6 The Population Lifetime . . . . . . . . . . . . . 67

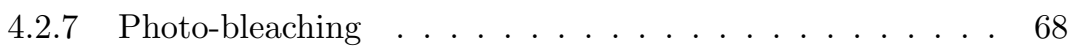

4.3 Experimental Set-Up . . . . . . . . . . . . . . . . 68

4.3.1 Raman Spectrometers . . . . . . . . . . . . . . . . 69

4.3 .2 Cryostats . . . . . . . . . . . . . . 73

4.3.3 Sample Preparation . . . . . . . . . . . . . . . . . 74

4.4 Anharmonic Coupling and Lifetimes _ . . . . . . . . . . 76

4.4.1 Anharmonic Coupling of Vibrational States . . . . . . . 76

4.4.2 Vibrational Density of States . . . . . . . . . . . . 79

4.4 .3 Bath Interactions . . . . . . . . . . . . . . . . . . 80

4.4.4 Investigating the Anharmonic Coupling through the Temperature Dependence . . . . . . . . . . . . . . . . 81

4.4.5 Experimental Results . . . . . . . . . . . . . . . 83

4.5 The Plasmon Dispersion and Two Laser Pumping . . . . . . 86

4.5.1 The Theory of Two Laser Pumping . . . . . . . . . 87

4.5.2 Experimental Results . . . . . . . . . . . . . . . . 90

4.5.3 Limitations and Applications for Two Laser Pumping . . 93

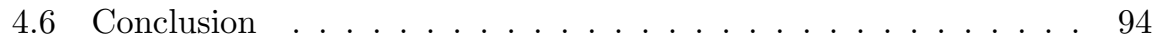

5 Wavelet Tranforms and Backgrounds $\quad 96$

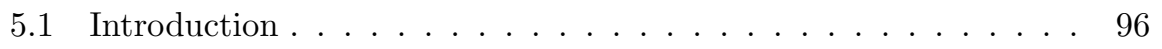

5.2 The Theory of Wavelet Transforms . . . . . . . . . . . . 98

5.2.1 Wavelet Transforms . . . . . . . . . . . . . . . 98

5.2 .2 Types of Wavelet Transforms . . . . . . . . . . . . 102

5.2.3 The Mallat Pyramidal Algorithm . . . . . . . . . . . . . . 104

5.3 Wavelet Transforms in Signal Processing . . . . . . . . . . . . . 110

5.3.1 The Three Frequency Regimes . . . . . . . . . . . 110 
5.3.2 Signal Modification using Wavelet Transforms . . . . . . . 112

5.4 The Iterative Algorithm . . . . . . . . . . . . . . . . 115

5.4 .1 The Iterative Algorithm . . . . . . . . . . . . . . 116

5.4 .2 The COBRA . . . . . . . . . . . . . . . . . . . 119

5.4 .3 A Simulated Signal . . . . . . . . . . . . . . . . . 122

5.4.4 SERS Applications for the COBRA . . . . . . . . 126

5.4.5 A Comparison with other Wavelet Based Algorithms . . . 131

5.5 Conclusion . . . . . . . . . . . . . . . . . . . . . 131

6 Single Molecule Vibrational Pumping 133

6.1 Introduction . . . . . . . . . . . . . . . . . . . . 133

6.2 Single Molecule Pumping Theory . . . . . . . . . . . . . . . . . 134

6.3 Initial Observations . . . . . . . . . . . . . . . . . . . . 138

6.3.1 The Two Analyte SERS Technique . . . . . . . . . . 138

6.3.2 Experimental Observations of Single Molecule Vibrational

Pumping . . . . . . . . . . . . . . . . . . . 142

6.4 A Statistical Analysis of the Pumping Cross-section . . . . . . 147

6.4.1 Experimental Method . . . . . . . . . . . . . . . . 147

6.4.2 Single Molecule Events . . . . . . . . . . . . . . . . . . . 148

6.4.3 The Pumping and SERS Cross-Sections . . . . . . . . . . 151

6.5 The Non-Radiative Enhancement Factor . . . . . . . . . . . . 159

6.5.1 SEF and SERS Comparisons . . . . . . . . . . . . 159

6.5.2 Experimental Method . . . . . . . . . . . . . . . . 161

6.5.3 Single Molecule Events . . . . . . . . . . . . . . . . . . 162

6.5.4 The Quantum Yield of Crystal violet . . . . . . . . . 165

6.5.5 A Comparison between $M_{\text {Tot }}$ and $M_{\text {Rad }} \ldots \ldots \ldots . .167$

6.5.6 A Return to the Single Molecule Pumping Cross-Section . 170

6.6 Conclusion . . . . . . . . . . . . . . . . . 171

$\begin{array}{lll}7 & \text { Conclusions } & 173\end{array}$

$\begin{array}{ll}\text { A Cross-Sections } & 179\end{array}$

$\begin{array}{ll}\text { B Measuring Scattering Volumes } & 181\end{array}$

$\begin{array}{ll}\text { C Measuring the SERS Cross-Section } & 185\end{array}$

$\begin{array}{ll}\text { D Published Papers } & 187\end{array}$ 


\section{Chapter 1}

\section{Introduction}

\section{Motivation and Goals}

Initially, we had a goal to continue the research that was begun by Maher et al. [1], in our laboratory, in the area of vibrational pumping in SERS. This included addressing the questions:

- How can we accurately measure the vibrational lifetime?

- Is the measured pumping cross-section a good representation of the SERS cross-section? While the SERS cross-section only contains a contribution from radiative Stokes scattering, the pumping cross-section also has a contribution from non-radiative Stokes scattering.

- What effect does the dispersion in the plasmon resonance have on the cross-section estimate?

With these problems in mind, I began a series of extensive high resolution temperature dependent measurements. This often involved days of sitting in the dark, taking temperature dependent measurements of SERS spectra. During this time we addressed the first problem but we struggled to reach any conclusions on the final two.

Sometime during my second year of $\mathrm{PhD}$ research, the laboratory had to be shut-down for several months so that refurbishments could be made. During this time I took the opportunity to address a problem that is common in many spectroscopic applications: background removal. I was drawn to this problem for several reasons: 
- it gave me an opportunity to practice a skill that I enjoy but have very little experience with: programming;

- the theory of wavelet transforms was very appealing;

- the problem was not unique to SERS.

This project consisted of creating a MatLab ${ }^{\mathrm{TM}}$-based program which utilised wavelet transforms to accurately fit the background. For our purposes, the attraction of an algorithm for background removal came from the fact that we could use it to separate the signal contribution due to fluorescence and Raman scattering. There were some difficulties associated with this project, however, which were mainly due to our lack of knowledge in the field of signal processing and wavelet transforms in particular. After some period of learning though, we managed to produce an automated algorithm for background subtraction using wavelet transforms, which is freely available and fully explained in a companion publication in Applied Spectroscopy [2]. This publication turned out to be very important for the final aim of the thesis: the origin of the non-radiative cross-section. This project required accurate estimates of fluorescence and Raman intensities for thousands of spectra which could only be achieved with this algorithm.

Soon after we returned to the laboratory, we made our first observations of single molecule vibrational pumping [3]. These observations were very important as they allowed us to measure both the pumping and SERS cross-section simultaneously. It was found that the two cross-sections were in fact different and that there was a substantial effect due to the dispersion in the plasmon resonance. While these results addressed some of our original problems, they did not explain why the cross-sections were different.

We hypothesised that the discrepancy was due to non-radiative Stokes scattering increasing the vibrational population but not being observed as a Stokes event (as the Stokes photon was not detected). To prove this was the case, the final months of my $\mathrm{PhD}$ research consisted of taking single molecule SERS spectra and separating them into their fluorescence and Raman components (using our algorithm). By comparing the relative intensities of these two processes, it was possible to estimate both the radiative and non-radiative SERS cross-sections and provide a completely new insight into the SERS process. 


\section{Thesis Outline}

The thesis will begin with a chapter on the interaction of light with matter (chapter 2) specifically fluorescence and Raman scattering. In this chapter I will discuss the basic states of a molecule and how photons interact with them.

This discussion will be extended in chapter 3 to molecules that are located close to metallic nano-particles. The theory of how the fluorescence and Raman scattering intensity is modified by the presence of the metal will be given. Furthermore, I will discuss how effective different nano-particle structures are for enhancing the SERS signal.

Chapter 4 will introduce the concept of vibrational pumping and how it can be used to estimate pumping cross-sections. An investigation into the anharmonic coupling between vibrational modes will be given along with an explanation of how we can use that knowledge to accurately measure vibrational lifetimes. In addition, I will describe how it is possible to observe the dispersion in the plasmon resonance distribution using a novel technique involving vibrational pumping, in which the population is excited with one laser and observed with another (two laser pumping).

Wavelet transforms and an explanation of why they are effective for fitting spectroscopic backgrounds will be given in chapter 5. An algorithm (developed in this thesis) will be described which involves iteratively performing a discrete wavelet transform to obtain the best possible background fit. Also included will be a demonstration of the algorithm's effectiveness using simulated and real SERS spectra with varying backgrounds.

Finally the results of the single molecule vibrational pumping measurements will be presented in chapter 6 . An overview of single molecule SERS will be given and the pumping theory will be adapted for single molecule events. Chapter 6 will also include an investigation into the effect of non-radiative processes on SERS and fluorescence for molecules that are directly adsorbed onto the surface of silver nano-particles.

\section{The Background of Vibrational Pumping}

Vibrational pumping was first proposed by Kneipp et al. in 1996 [4]. Kneipp presented results showing the variation in the anti-Stokes and Stokes intensity ratio with the incident laser power. Kneipp observed that the ratio increased linearly with power and theorised that the SERS cross-section could be esti- 
mated from the slope. However, the results were met with a lot of scepticism from the SERS community due to the extremely large enhancement factors they predicted. While the measured cross-sections were slightly larger than expected, the large enhancement factor estimation was mainly due to the crosssection being normalised with respect to a non-resonant molecule, which has a much smaller bare Raman cross-section than the molecule actually observed. In addition, the results were criticised for their inability to rule out the effect of laser heating. The results presented in Kneipp's paper were taken at room temperature, where the pumped population can be several orders of magnitude smaller than the population that is created through thermal excitation. At these temperatures, an increase in the incident laser power will increase the effective temperature of the observed molecules, which will subsequently increase the thermal population. The variation of the anti-Stokes to Stokes intensity ratio with laser power was predicted to vary in the same manner for both laser heating and vibrational pumping.

In 2006, Maher et al. presented observations of anti-Stokes scattering at very low temperatures $\sim 10 \mathrm{~K}[5-8]$. At these temperatures, the thermally excited population should be well below the noise level and anti-Stokes scattering should not be observable unless there is an additional population created through another mechanism, i.e. vibrational pumping. In addition, they showed that the variation in the anti-Stokes to Stokes intensity ratio varied with temperature in exactly the same way as predicted if vibrational pumping is present. It was again proposed that the SERS cross-section could be predicted from the fitting parameters. However, the measured cross-sections were similar to those predicted by Kneipp et al. It was much more difficult for the SERS community to discount these measurements as laser heating is not important at low temperatures. As a result, the assumption that the pumping cross-section was the same as the SERS cross-section became questionable. In fact, the resolution of this problem is one of the major results of this thesis. 


\section{Chapter 2}

\section{The Interaction between Light and Matter}

The aim of this chapter is to give an introductory theoretical overview of the interaction between light and matter. A description of the energy states of a molecule and possible transitions will be given including an explanation of vibrational modes. Furthermore, a brief overview of the classical theory of Raman scattering will be provided along with how it is modified with the semi-classical theory. Finally, a discussion of absorption and fluorescence spectroscopy will be presented, including an explanation of why it is important in Raman scattering.

\subsection{Molecular Transitions}

As we know from quantum mechanics, a molecule has discrete energy levels that it may exist in. For a single atom, there are only electronic states available (ignoring energy states of the nucleus). However, for a polyatomic molecule there are also vibrational and rotational states. These states are coupled to the electronic states of the molecule, as the occupied electronic orbitals will affect the binding strength between nuclei and modify the vibrational energy. The energy required to excite a molecule between vibrational states is of the order of $\sim 100 \mathrm{meV}$, which is in the infrared (IR) wavelength range for excitation by direct absorption. The electronic transitions, on the other hand, are much larger in energy $(\sim 1 \mathrm{eV})$ and often excitable by direct absorption in the visible wavelength range $(400-700 \mathrm{~nm})$. 


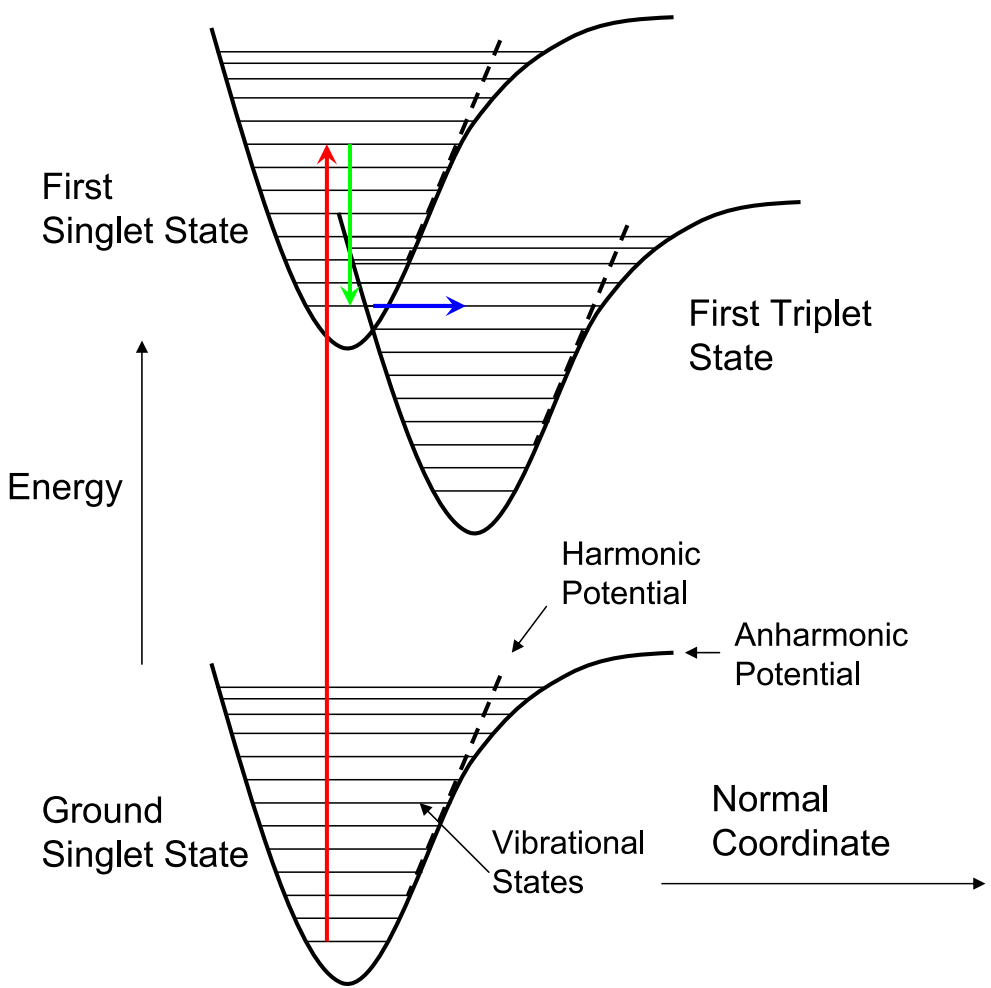

Figure 2.1: An energy diagram of a simplified molecule as described in the text. The energy is plotted as a function of the normal coordinate of the vibration. Three possible transitions are shown, an excitation to the first singlet state from the ground state (red arrow), the relaxation of the molecule to the bottom of the first singlet state, and the inter-system crossing transition from the first singlet state to the first triplet state.

At low amplitudes, the vibrational modes can be approximated as harmonic oscillators. However, as the amplitude increases, the structure of the molecule is distorted and the vibration becomes anharmonic [9-11]. If we take the simplified case of a molecule with three electronic states and one vibrational mode (with many quantum levels), the molecular energies can be pictured as shown in figure 2.1 .

For the work carried out in this thesis, there are two types of electronic states that are important, singlet and triplet. Singlet states have a total spin of zero while a triplet state has a total spin of one. It is often the case that a singlet and a triplet state will partially overlap (as shown in figure 2.1), however, the molecule cannot easily switch between them. Typically, a molecule will make a transition between states by emitting or absorbing a photon. However, a photon cannot 
solely facilitate a transition between the singlet and triplet states as it cannot flip the spin of an electron. Nevertheless, the molecule may undergo a radiationless transition by reversing the spin of an electron via external interactions, but this has a low probability of occurring for fluorescent molecules [10]. This is known as inter-system crossing. For each electronic state there are many vibrational levels which correspond to different amplitudes of the same vibration.

\subsection{Molecular Vibrations}

A vibrational or rotational mode is the movement of the particles within a molecule without perturbing the centre of mass. Each atom has 3 degrees of freedom which it can move in. A molecule consisting of $N$ atoms will therefore have $3 N$ total freedoms of motion. However, three of these freedoms will be translational freedoms of the molecule resulting in a movement of the centre of mass. For a non-linear molecule there are also three rotational freedoms. The remaining degrees of freedom must therefore correspond to deformations of the molecule, of which there will be a total of $3 N-6$. For a linear molecule, we lose one of the rotational freedoms and instead have $3 N-5$ vibrational modes.

For small molecules consisting of two or three atoms, it is relatively straightforward to determine the possible vibrational modes. In the case of a diatomic molecule (two atoms) there must be one vibration (as it is linear). This vibration is shown in figure 2.2(a) and consists of the two atoms moving in opposite directions along the bond axis. The relative movement of each atom will depend on the difference in their masses. For a triatomic molecule like water $\left(\mathrm{H}_{2} \mathrm{O}\right)$ there must be three vibrational modes. The vibrational modes are shown in figures 2.2(b)-(d). For larger molecules it becomes much more difficult to determine the vibrational frequencies and techniques such as density functional theory (DFT) [12-14] are often used to calculate them from the electronic structure.

Each of the vibrational modes have an energy associated with them which is proportional to the frequency of the vibration. It is common in Raman spectroscopy to refer to a Raman Shift which are in units of wavenumbers $\left(\mathrm{cm}^{-1}\right)$ and proportional to the frequency of the vibration:

$$
\Delta \nu=\frac{E_{L}-E_{S}}{h c}=\frac{\omega_{\nu}}{2 \pi c},
$$

where $\omega_{\nu}$ is the angular frequency of the vibration, $E_{L}$ is the energy of the incident photons and $E_{S}$ is the energy of the scattered light. The rotational 


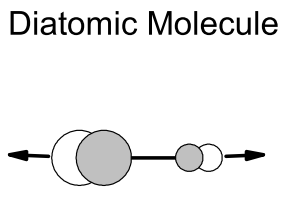

(a)

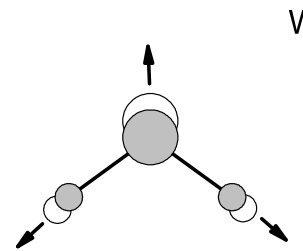

(b)
Water-like Triatomic Molecule

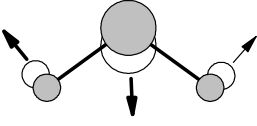

(c)

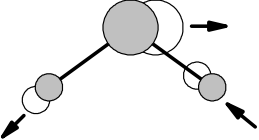

(d)

Figure 2.2: The vibrational modes of (a) a diatomic molecule, and (b)-(d) a nonlinear water-like triatomic molecule.

modes all have relatively small energies being below $100 \mathrm{~cm}^{-1}$. The vibrational modes are all found in the region between $0-3500 \mathrm{~cm}^{-1}$. However, apart from a few exceptions (including hydrogen mode and triple bonds), most of the vibrations are less than $1650 \mathrm{~cm}^{-1}$ in organic molecules.

There are several characteristic vibrations that have predictable vibrational energies:

Hydrogen Stretching Modes These are localised vibrations within the molecule that involve the displacement of a hydrogen atom with respect to its surroundings [15]. Because the mass of hydrogen is so small, the frequency of these kinds of vibrations are relatively large. Hydrogen stretching modes have an energy around $\sim 3000-3500 \mathrm{~cm}^{-1}$ which is isolated from the other vibrational energies.

Breathing Modes These are found in molecules that have carbon rings, most often Benzene rings. The overall movement of the atoms that form the ring is radial, resulting in an increase and decrease in the radius of the ring. These vibrations typically have energies around $\sim 1500-1600 \mathrm{~cm}^{-1}$.

Triple Bond Modes These are localised vibrations that involve stretching of the triple bonds. As with the hydrogen stretching modes, these are high energy vibrations and are found in the region between the breathing modes and the hydrogen stretching modes $\sim 2000 \mathrm{~cm}^{-1}$. Triple bond modes are typically the only vibrations found in this region.

Most of the molecules used in this thesis can be thought of as having a dense region of vibrational modes between $0-1650 \mathrm{~cm}^{-1}$, and a small region close to $3000 \mathrm{~cm}^{-1}$ that corresponds to hydrogen stretching modes. None of the observed molecules have triple bonds but they do have breathing modes. 
Breathing modes are often ideal for Raman spectroscopy due to their large cross-sections.

Once a vibration is created, it will not remain indefinitely. Due to the anharmonic nature of the vibrations, they interact with each other. This is known as anharmonic coupling [9]. A vibration will therefore decay by transferring its energy to lower energy vibrations that it is coupled to, as long as energy is conserved during the process. It is also possible for a vibration to decay via interactions with other molecules. The interaction between vibrations will be re-addressed in section 4.4 .

\subsection{The Raman Effect}

Rayleigh [16] and Raman scattering [17] are two-photon instantaneous processes in which the incident photon does not need to correspond to a possible transition of the molecule [18-20]. Consequently, these types of scattering can occur at any frequency. However, the probability that a photon will be scattered in this way will be wavelength dependent. In the case of Rayleigh scattering, the scattered photon has the same energy as the incident photon. The state of the molecule is therefore unchanged. Raman scattering, on the other hand, is an inelastic process and the scattered photon will have either less or more energy than the incident photon. As a result, the state of the molecule will be different. In order to conserve energy, the difference in energy between the incident and scattered photons must be equal to a molecular transition within the molecule. Figure 2.3 shows a Jablonski diagram of Rayleigh and Raman scattering interacting with a virtual state which is possible as the process is instantaneous.

There are two types of Raman scattering processes, Stokes and anti-Stokes scattering. In the former case, the molecular energy is increased and the scattered photon will have less energy than the incident photon. For anti-Stokes scattering, the scattered photon has more energy than the incident photon and the molecular energy is decreased. In order for anti-Stokes scattering to occur, the molecule must already be in an excited vibrational state. Consequently, the probability that anti-Stokes scattering will occur is proportional to the average population of the vibrational mode. 


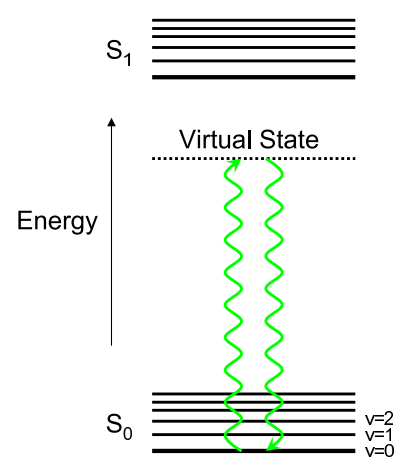

(a) Rayleigh Scattering

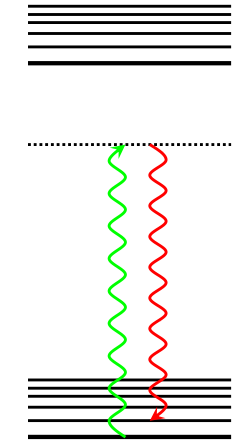

(b) Stokes Scattering

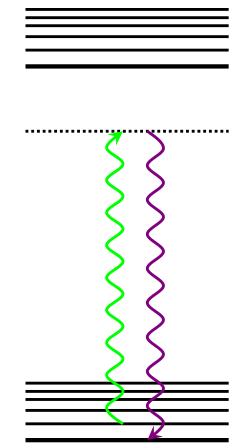

(c) anti-Stokes Scattering

Figure 2.3: Jablonski diagrams of (a) Rayleigh scattering, (b) Stokes Raman scattering, and (c) anti-Stokes Raman scattering. The wiggly arrows correspond to photonic processes in which either a photon is destroyed (upward arrow) or created (downward arrow). This will be the case for all Jablonski diagrams shown in this thesis.

\subsubsection{A Classical Theory of Raman and Rayleigh Scatter- ing}

There are three treatments for building a mathematical theory of Raman and Rayleigh scattering: a completely classical treatment in which the electromagnetic radiation and material are treated classically, a semi-classical treatment in which the electromagnetic radiation is treated classically but the material is treated as a quantum mechanical system, and a fully quantum mechanical treatment with both the radiation and material treated with quantum mechanics. For a full description of these theories I direct the reader to literature such as [19] which gives a full overview of these treatments. Here I will give a brief overview of the classical treatment and describe how it is modified by taking into account the quantum mechanical nature of the system.

In the classical and semi-classical treatments, the system is considered to be the oscillating electric and magnetic multipole moments induced in the molecule by the electromagnetic field of the incident light waves. The oscillating electric dipole contribution is the dominant term with the other multipole terms being several orders of magnitude smaller. We therefore restrict ourselves to assuming that the molecule acts like an oscillating electric dipole [21]. The time-averaged power per unit solid angle radiated by the molecule, which is induced by the electric field of the incident radiation with frequency $\omega_{L}$, is therefore given by: 


$$
I=\frac{\omega_{S}^{4} p_{0}^{2} \sin ^{2} \theta}{32 \pi^{2} \epsilon_{0} c^{3}},
$$

where $p_{0}$ is the amplitude of the induced electric dipole, $\omega_{S}$ is the frequency of the dipole, and $\theta$ is the angle in spherical coordinates representing the colatitude with the dipole pointing along the $\theta=0$ axis. Consequently, $\theta$ can vary between 0 and $\pi$. The objective of the different theories is to find how the values of $\omega_{S}$ and $p_{0}$ are determined by the properties of the molecule and the radiation.

For the case of the classical treatment, the electric dipole moment can be approximated, to first order, as being linearly dependent on the incident electric field:

$$
\mathbf{p}=\alpha \cdot \mathbf{E},
$$

where $\alpha$ is the linear polarizability tensor of the molecule. If the molecule is free to vibrate around an equilibrium position, the variation of the polarizability due to the vibration can be expressed by expanding each component of $\alpha$ by a Taylor series with respect to the normal coordinates of the vibration as:

$$
\alpha_{a b}=\left(\alpha_{a b}\right)_{0}+\sum_{k}\left(\frac{\partial \alpha_{a b}}{\partial Q_{k}}\right)_{0} Q_{k}+\ldots,
$$

where the $(\ldots)_{0}$ corresponds to the value in the brackets at the equilibrium position, and $Q_{k}$ are the normal coordinates of the molecular vibration with frequency $\omega_{k}$. There are also higher order terms but they are several orders of magnitude smaller and will be ignored at this time. Assuming simple harmonic motion of the vibration:

$$
Q_{k}=Q_{k 0} \cos \left(\omega_{k} t+\phi_{k}\right),
$$

and an electric field of the form:

$$
\mathbf{E}=\mathbf{E}_{0} \cos \left(\omega_{L} t\right)
$$

the electric dipole moment can be written as:

$$
\begin{aligned}
\mathbf{p}= & \mathbf{p}^{\mathrm{Ray}} \cos \left(\omega_{L} t\right) \\
& +\mathbf{p}^{\mathrm{Ram}}\left[\cos \left(\left(\omega_{L}-\omega_{k}\right) t-\phi_{k}\right)+\cos \left(\left(\omega_{L}+\omega_{k}\right) t+\phi_{k}\right)\right],
\end{aligned}
$$




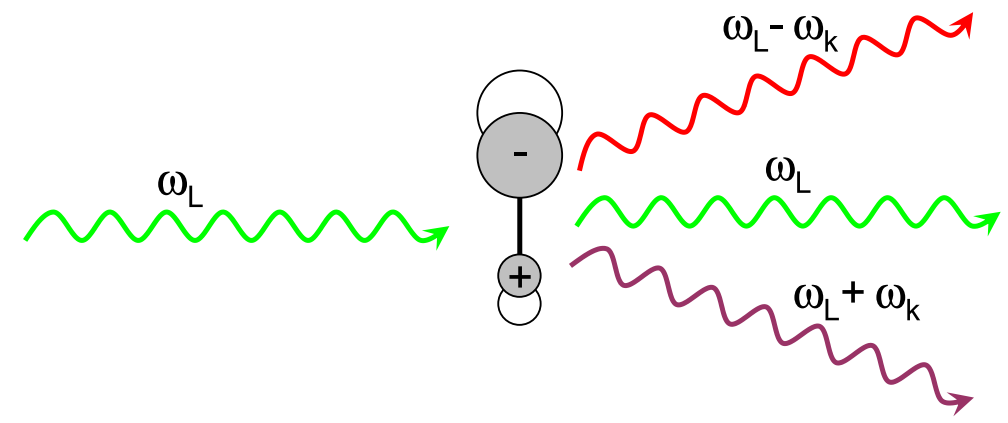

Figure 2.4: Incident light of frequency $\omega_{L}$ being scattered by an electric dipole vibrating at a frequency $\omega_{k}$. The scattered light will either have a frequency of $\omega_{L}$ (Rayleigh scattering), $\omega_{L}-\omega_{k}$ (Stokes Raman scattering), or $\omega_{L}+\omega_{k}$ (anti-Stokes Raman scattering).

where:

$$
\mathbf{p}^{\text {Ray }}=\alpha_{\mathbf{0}} \cdot \mathbf{E}_{\mathbf{0}},
$$

and

$$
\mathbf{p}^{\mathrm{Ram}}=\frac{1}{2} Q_{k}\left(\frac{\partial \alpha}{\partial Q_{k}}\right)_{0} \cdot \mathbf{E}_{\mathbf{0}} .
$$

The molecule which is perturbed by a vibration therefore has three frequency components in the electric dipole moment: a component with frequency $\omega_{L}$ which corresponds to Rayleigh scattering, a component with frequency $\omega_{L}-\omega_{k}$ which corresponds to Stokes Raman scattering, and a component with frequency $\omega_{L}+\omega_{k}$ which corresponds to anti-Stokes Raman scattering. Figure 2.4 shows the type of scattering that will occur for a dipole that is vibrating with a frequency $\omega_{k}$.

Taking a closer look at equations 2.7-2.9 we see that Rayleigh scattering can only occur if the polarizability tensor (at the equilibrium position) is non-zero in at least one component, which is always the case. Raman scattering, however, requires that the derivative of the polarizability tensor, with respect to the normal coordinate of the vibration, is non-zero in at least one component. This may not always be the case and some vibrational modes may not be observable with Raman scattering. This is what defines the Raman selection rules in the classical treatment. At this point it is useful to define the Raman tensor for a single vibration with frequency $\omega_{k}$ as: 


$$
\alpha_{k}^{\prime}=\left(\frac{\partial \alpha}{\partial Q_{k}}\right)_{0} .
$$

If this tensor is non-zero, the vibration is referred to as "Raman active". It is interesting to note that a vibration may still be excitable through direct absorption in the IR wavelength range even if the vibration is not Raman active. In contrast to Raman scattering, IR absorption may occur for a certain vibration as long as the derivative of the electric dipole moment is non-zero at the equilibrium position. For most SERS probes, the majority of the vibrational modes will be both Raman and IR active, but some will be more susceptible to IR scattering than Raman and vice versa. Consequently, the spectrum observed under an IR excitation may be substantially different to that observed under an excitation that is in the visible wavelength range. If the molecule has a centre of inversion, the Raman and IR spectra are mutually exclusive. This is a well known result of group theory which will not be expanded here any further.

Using the theory outlined in this section, and knowledge of the vibrational states and polarizability tensor, it is possible to calculate the Raman tensor for each vibration using equation 2.10 and determine which are Raman active.

\subsubsection{Limitations of the Classical Theory}

There are several limitations to the classical theory:

- It does not account for rotational modes of the molecule.

- It assumes prior knowledge of the polarizability tensor and the vibrational modes of the molecule. However, these can only be estimated with a quantum mechanical understanding of the properties of the molecule. A popular technique for calculating these properties is DFT [22].

- The frequency dependence of Rayleigh scattering is correct, but not necessarily for Raman scattering especially at frequencies close to an electronic transition where resonance effects start playing a role.

- The Stokes intensity predicted in the classical theory does not account for spontaneous creation of a vibration. Furthermore, the classical theory does not correctly address anti-Stokes scattering.

- The Taylor expansion in equation 2.4 is not valid when the incident frequency coincides with an electronic transition (resonance phenomena). 


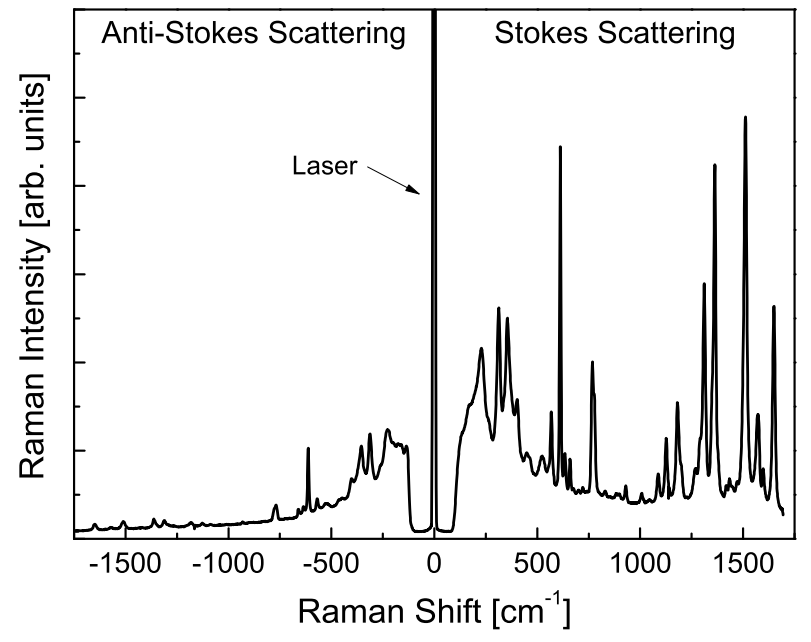

Figure 2.5: A surface-enhanced Raman spectrum of Rhodamine 6G taken at room temperature $(\sim 300 K)$. The central peak corresponds to light scattered at the laser frequency (Rayleigh scattering). A positive Raman shift corresponds to scattered light with less energy (Stokes scattering), and a negative Raman shift corresponds to scattered light with more energy (anti-Stokes scattering).

However, the classical treatment (with its limitations) is enough to understand the phenomena described in this thesis.

\subsubsection{Stokes and anti-Stokes Scattering}

In the semi-classical treatment, Stokes scattering occurs when the interaction results in the excitation of a vibration within the molecule. Consequently, the scattered light has less energy than the incident light. Anti-Stokes scattering, on the other hand, occurs when the interaction results in the absorption of a vibration that is already present. The scattered light therefore has more energy than the incident light. Figure 2.5 shows an example of a "surface-enhanced" Raman spectrum for the Rhodamine $6 \mathrm{G}$ molecule. Each of the peaks correspond to scattering via a Raman active vibrational mode of the molecule. It can be shown in the semi-classical theory of Raman that the Stokes and anti-Stokes intensities, for each mode, are related to the cross-section via:

$$
\begin{gathered}
I_{S}=N(1+n) \frac{d \sigma_{S}}{d \Omega} P_{\mathrm{Inc}} \\
I_{a S}=N n \frac{d \sigma_{a S}}{d \Omega} P_{\mathrm{Inc}}
\end{gathered}
$$


where $d \sigma_{S} / d \Omega$ is the differential Stokes Raman cross-section, $d \sigma_{a S} / d \Omega$ is the differential anti-Stokes Raman cross-section, $n$ is the ensemble-averaged vibrational population of the mode, and $N$ is the number of molecules being observed. The differential Raman cross-section is an intrinsic property of the molecule, which represents the probability that Raman scattering will occur for a specific incident frequency (see appendix A for a discussion on cross-sections). Typically the cross-section will vary with $\omega^{4}$ due to the electric dipole nature of the molecule. However, if the frequency of the incident radiation is close to an electronic transition in the molecule, the cross-section will be much larger. This is called resonant Raman scattering and will be discussed later in the chapter. In addition, the cross-sections are orientationally averaged.

Because the Stokes and anti-Stokes scattered light are different in frequency, the cross-sections will rarely be the same. This will become more pronounced for the larger energy vibrations, as the frequency difference between the antiStokes and Stokes scattered photons is much larger than for the low energy vibrations. Theoretically it is possible to derive the Raman cross-section from the structure of the molecule. However, due to the complexity of the molecules often measured, this may not be possible and other techniques are necessary. A common technique for measuring the cross-section is to compare the Raman intensity of the molecule with the intensity of another molecule, that has a known cross-section, under the same experimental conditions. This is explained in appendix $\mathrm{C}$.

The ensemble-averaged vibrational population is a measure of the average vibrational level that all of the molecules exist in. For example, if we consider a single vibrational mode (as in figure 2.3), a value of $n=2$ will mean that, on average, the molecules will be in the $v=2$ vibrational state. While each molecule must have a discrete vibrational population at any one time, the ensemble-averaged vibrational population can be any positive real number. Most molecules have many vibrational modes and the vibrational population will depend on the vibration being observed. At typical experimental temperatures $(\sim 300 \mathrm{~K})$, the dominant mechanism of vibrational excitation is through thermal effects. However, it can be shown that the thermally excited population is much smaller than one at these temperatures and typical vibrational energies (see section 2.1).

One might expect that the Stokes intensity in equation 2.11 will be independent of the vibrational population but there is a modification due to stimulated scattering. However, because $n<<1$ for most cases, we can simplify equation 
2.11 to:

$$
I_{S} \cong N \frac{d \sigma_{S}}{d \Omega} P_{\text {Inc }}
$$

\subsubsection{Types of Raman Scattering}

There are selection rules in photon scattering that govern the likelihood that a certain transition will occur as a scattering process. The most likely scattering mechanism is Rayleigh scattering for situations where there is no absorption. Raman scattering, resulting in a transition between adjacent states (for example, from the $v=1$ to the $v=2$ state), is the second most likely process but this is several orders of magnitude weaker than Rayleigh scattering. Additionally, there are higher order Raman processes $[23,24]$ that are much rarer but can be observed under certain situations.

\section{Overtones and Combinations}

Overtones and combinations are multi-phonon Raman processes and correspond to the higher order terms in equation 2.4.

For overtones, the frequency of the phonons are the same and, consequently, the transition involves a single vibrational state that jumps between non-adjacent levels. Figure 2.6 shows a Jablonski diagram of an overtone Raman process. The probability that an overtone process will occur is related to the difference in the phonon number, $\Delta v$. As a result, an event with $\Delta v=2$ is much more likely than an event with $\Delta v=3$.

For combinations, the phonon frequencies are different and multiple vibrations are excited simultaneously. This becomes rarer as the number of excited vibrations increases. As a result, the most common combination process will create two vibrations. If the vibrations have a frequency of $\omega_{k}$ and $\omega_{p}$, the Stokes scattered photon will have a frequency of $\omega_{S}=\omega_{L}-\omega_{k}-\omega_{p}$, where $\omega_{L}$ is the frequency of the incident light.

It is also possible to have anti-Stokes scattering of overtones and combinations. Furthermore, it is possible to have difference processes in which a vibration is absorbed (similar to an anti-Stokes process) and a second one is excited simultaneously. In this case the scattered light will have a frequency of $\omega_{S}=\omega_{L}+\omega_{k}-\omega_{p}$, where $\omega_{k}$ is the frequency of the absorbed vibration, and $\omega_{p}$ is the frequency of the excited vibration. Unlike overtones and combinations, 
$\mathrm{S}_{1}$
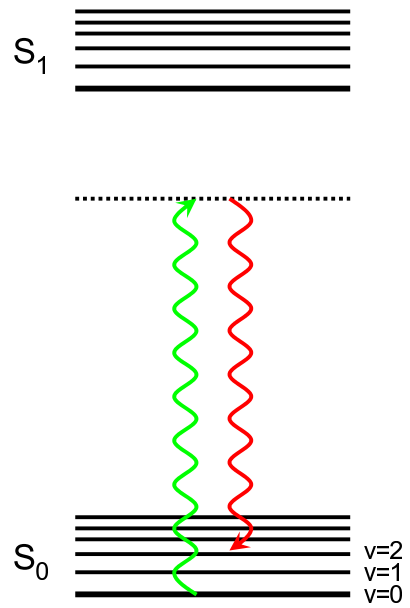

Figure 2.6: Jablonski diagram of overtone Raman scattering in which the molecule is excited from the $v=0$ state to the $v=2$ state.

difference scattering will depend on temperature as a vibrational mode must first be populated.

\section{Hyper-Rayleigh and Hyper-Raman Scattering}

Hyper-Rayleigh and hyper-Raman scattering are multiple photon processes and come from the non-linear electric field terms in the electric dipole moment which become important only at large laser powers:

$$
\mathbf{p}=\alpha \cdot \mathbf{E}+\beta \cdot \mathbf{E E}+\gamma \cdot \mathbf{E E E}+\ldots
$$

For first order hyper-Raman scattering, it is the hyper-polarizability tensor, $\beta$, that determines the hyper-Raman tensors. Hyper-Rayleigh and hyper-Raman scattering consist of two incident photons undergoing a single scattering process with a single scattered photon as shown in figure 2.7(a) and (b). In the case of hyper-Rayleigh scattering (see figure 2.7(a)), the scattered photon has twice the frequency of the incident light, $\omega_{S}=2 \omega_{L}$, while for hyper-Raman scattering (see figure $2.7(\mathrm{~b})$ ) with a vibration of frequency $\omega_{k}$, the scattered photon has a frequency of $\omega_{S}=2 \omega_{L}-\omega_{k}$.

It is also possible to have scattering of three incident photons called secondorder Hyper-Raman (or Rayleigh), scattering. This involves the second-order polarizability tensor, $\gamma$. Similarly, there is higher order hyper-Raman scattering with even larger numbers of incident photons. However, higher order hyper- 


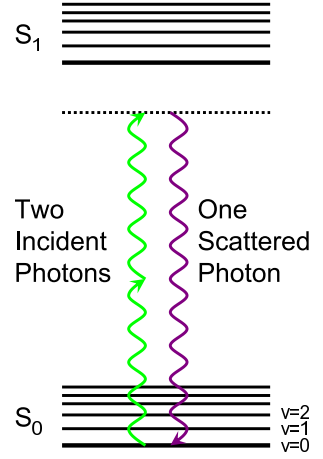

(a) Hyper-Rayleigh

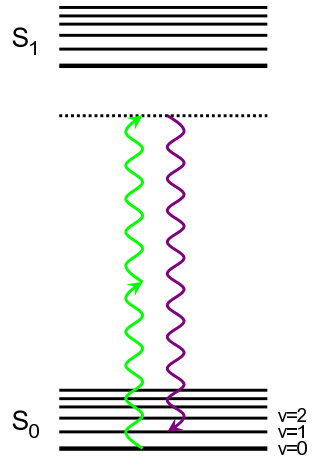

(b) Hyper-Raman

Figure 2.7: Jablonski diagram of (a) hyper-Rayleigh scattering, and (b) hyper-Raman scattering.

Raman scattering is very weak and, in fact, there are very few cases of even first order hyper-Raman scattering (two incident photons) being observed [25].

\subsubsection{Resonance Raman Scattering}

For a simple electric dipole, the radiated intensity has a $\omega^{4}$ dependence. However, this approximation is no longer valid for molecules that are radiating close to an electronic transition (resonance condition). In this situation, a quantum treatment of the electronic structure of the molecule must be performed in order to estimate the linear polarizability. Without going into the details, the Raman cross-section is greatly enhanced close to this excitation frequency, resulting in a scattering intensity that may be many orders of magnitude larger than expected for an electric dipole. This is known as resonance Raman scattering as the excitation is in resonance with an electronic transition.

In resonance Raman scattering, the virtual state no longer exists and the incident light interacts with a real state of the molecule. It is this interaction that causes the large enhancement in the scattering intensity. Because of this effect, many Raman experiments are carried out on dye molecules which absorb light in the visible wavelength range. A typical visible laser can therefore take advantage of the intrinsic resonance properties of the molecule to obtain very detailed Raman spectra. The absorption spectrum of some of the dyes used throughout this thesis are shown in figure 2.8 and have been chosen as most of the experiments will be performed under resonant conditions.

There are however complications that arise when exciting molecules close to 


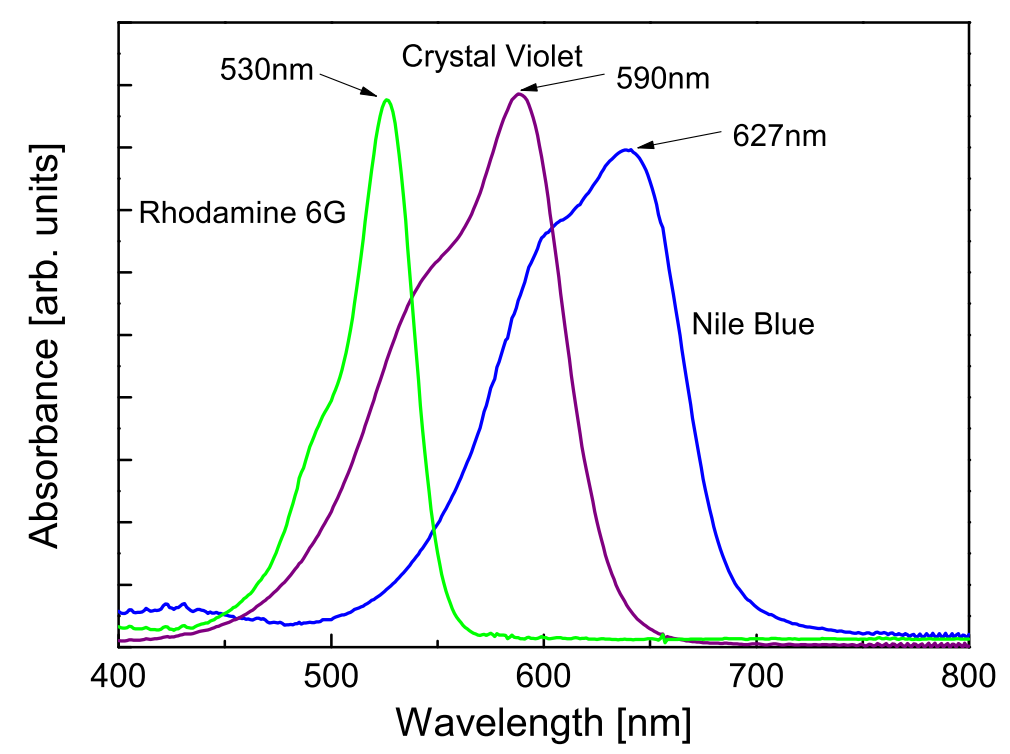

Figure 2.8: Absorbance spectra for Rhodamine 6G (green curve), Crystal Violet (violet curve) and Nile Blue (blue curve), in water. The curves have been scaled so that they are similar in height for ease of comparison. Noted are the wavelengths that correspond to maximum absorption for each dye.

the absorption resonance. One such complication is that the Raman tensors are modified under resonant conditions and become polarized along the same axis. Furthermore, the relative scattering intensities of the vibrational modes may be modified.

Another complication (from the point of Raman) that arises under resonant conditions is fluorescence. Fluorescence occurs when a molecule is excited to a higher electronic state. One of the ways that the molecule can return to the ground state is to emit a fluorescence photon. This will be discussed in more detail in the next section. By tuning the incident frequency to the electronic transition, we are not only allowing resonant Raman scattering to occur, but also fluorescence. Furthermore, even with the enhancement of the Raman crosssection, the fluorescence cross-section will typically be much larger (except in special cases) and spectrally overlapping. Consequently, the Raman signal may be drowned out by fluorescence and not observable.

Despite these complications, the cross-section enhancement is a very attractive property for a molecule. This is especially true in surface-enhanced Raman scattering where the effect of fluorescence can be reduced. Under these condi- 


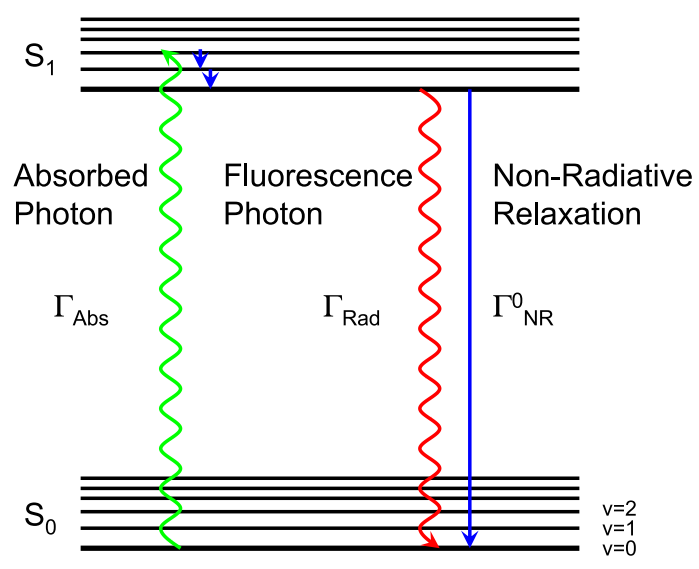

Figure 2.9: Jablonski diagram of fluorescence.

tions it is possible to increase the cross-section sufficiently that single molecules become observable. I will return to this topic in chapter 6 .

\subsection{Absorption and Fluorescence Spectroscopy}

\subsubsection{Decay Rates}

Let us consider the case in which the incident photon coincides with a transition between two singlet states. In this situation there is a high probability that the molecule will completely absorb the photon as shown in figure 2.9. At this point, the molecule will quickly decay to the minimum energy of the excited singlet state. This will typically occur within $\sim 0.1-10 \mathrm{ps}$ of the initial absorption for most polyatomic molecules [26]. In order for the molecule to return to the ground state, it must decay either by radiating a photon or non-radiatively by distributing the energy internally within the molecule or externally by interacting with nearby molecules (this is also known as intrinsic non-radiative decay). The former process is called fluorescence and the radiated light is called a fluorescence photon. Each of these processes has an associated decay probability rate which is the reciprocal of the average lifetime that the molecule will remain in the excited state before undergoing the associated relaxation process:

$$
\Gamma_{\mathrm{Rad}}=\frac{1}{\tau_{\mathrm{Rad}}}
$$

for fluorescence, and: 


$$
\Gamma_{\mathrm{NR}}^{0}=\frac{1}{\tau_{\mathrm{NR}}^{0}},
$$

for non-radiative decay. $\Gamma_{\text {Rad }}$ is the radiative decay rate which has a lifetime of $\tau_{\mathrm{Rad}}$, and $\Gamma_{\mathrm{NR}}^{0}$ is the intrinsic non-radiative decay rate which has a lifetime of $\tau_{\mathrm{NR}}^{0}$.

It is useful at this point to define the intrinsic quantum yield of the molecule as:

$$
Q^{0}=\frac{\Gamma_{\mathrm{Rad}}}{\Gamma_{\mathrm{Tot}}},
$$

where $\Gamma_{\text {Tot }}=\Gamma_{\mathrm{Rad}}+\Gamma_{\mathrm{NR}}^{0}$, and is known as the total decay rate. The intrinsic quantum yield is the probability that an absorbed photon will be re-emitted as a fluorescence photon and, consequently, must always be less than or equal to one.

\subsubsection{The Fluorescence Spectrum}

When a molecule fluoresces, the molecule will decay from the bottom of the excited singlet state to anywhere in the ground singlet state. As a result, the frequency of the fluorescence photons will cover a wide range of frequencies. Furthermore, because the vibrational states of the ground and excited singlet states are roughly the same, the absorption and fluorescence spectra have a similar shape, but inverted (see figure 2.10). Moreover, the fluorescent photons are Stokes-shifted (have less energy than the absorbed photons) which is clear as energy is lost when the molecule relaxes within the first excited state.

The overall fluorescence intensity (integrated over all scattering wavelengths) can be expressed in terms of the fluorescence cross-section as:

$$
I_{\text {Fluo }}=N \frac{d \sigma_{\text {Fluo }}}{d \Omega} I_{L},
$$

where the total fluorescence cross-section (integrated over all scattering directions) is related to the absorption cross-section and quantum yield by:

$$
\sigma_{\text {Fluo }}=Q^{0} \sigma_{\text {Abs }} \text {. }
$$

In the case of Raman scattering, it is advantageous to use molecules that have extremely small quantum yields. This is especially the case when observing resonant Raman scattering in which the fluorescence photons overlap with 


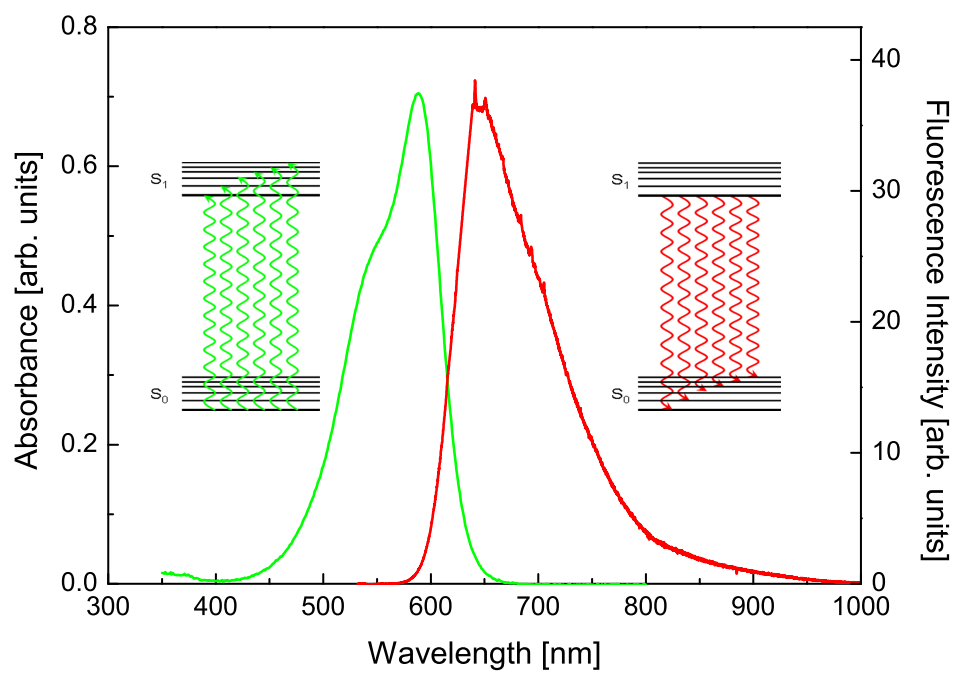

Figure 2.10: The absorbance (green curve) and fluorescence (red curve) spectrum at $633 \mathrm{~nm}$ for Crystal Violet. Due to thermal excitation of vibrations, there is an overlap between the two spectra. Furthermore, there are Raman peaks on top of the fluorescence spectrum which are observable due to the low quantum yield of Crystal Violet. Also included are Jablonski diagrams showing schematically some of the possible transitions for absorption and fluorescence. 


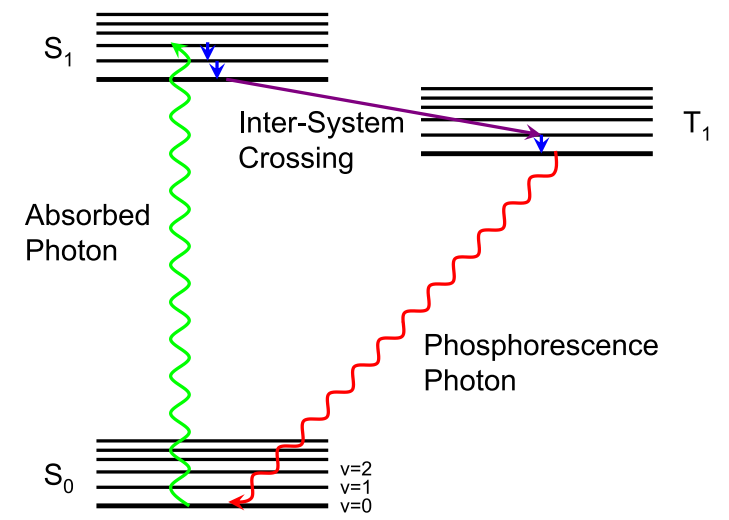

Figure 2.11: Jablonski diagram of Phosphorescence.

the Raman photons and may overwhelm the signal. By using a molecule with a small quantum yield, most of the excited molecules will relax via non-radiative processes and the Raman intensity may be observable above the fluorescence background. This becomes less of a problem in surface-enhanced Raman scattering as other, more efficient, non-radiative processes become available (this will be explained in the next chapter).

\subsubsection{Interactions with the Triplet State}

One of the mechanisms for internal relaxation of the molecule is via the interaction with the triplet state. A transition from the singlet state to the triplet state is generally forbidden. However, the energy of the first singlet state and the first triplet state overlap for many molecules as was shown in figure 2.1. As a result, spin-orbit or intramolecular interactions will often allow the molecule to undergo inter-system crossing to the triplet state $[10,11,26]$. At this point, the molecule is trapped and cannot easily relax to the ground singlet state. During this time, the molecule will no longer fluoresce but it may still undergo Raman scattering. This is known as "fluorescence blinking" $[27,28]$. The molecule may remain in this state for a relatively long time (as long as seconds) but it will eventually decay, either radiatively by emitting a photon or non-radiatively through other mechanisms. Radiative emission of this form is known as phosphorescence (see figure 2.11) and is possible due to additional interactions which facilitate the changing of the electron spin.

When the molecule is in a triplet state, it typically has a high chemical reac- 
tivity with oxygen $[29,30]$ which can often lead to the destruction of the molecule (also called photo-bleaching). As a result, the photo-bleaching rate will often depend on the transfer rate to the triplet state and the lifetime of the molecule when it is in the triplet state. Furthermore, photo-bleaching rates can generally be reduced with a reduction of oxygen content in the environment. On the other hand, non-resonant molecules have been observed to photo-bleach under SERS conditions and, consequently, there must be additional photo-bleaching mechanisms that are not well understood. 


\section{Chapter 3}

\section{The Surface Enhancement}

In the previous chapter I gave an overview of the theory of Raman scattering and fluorescence. This chapter will extend that discussion by investigating how fluorescence and Raman scattering are modified when the molecule is placed close to a metallic surface [9,31-38]. I will begin by introducing the concept of surface plasmon resonances which can be utilised to create extremely large localised electromagnetic fields. A discussion on how the fluorescence and Raman spectrum are modified for molecules positioned in these regions will then be presented. Finally, I will investigate how effective certain nano-particle systems are for creating these large localised fields.

\subsection{Surface Plasmon Resonances}

Surface plasmon resonances (SPRs) create the large Raman signals observed in SERS. It is therefore very important that we understand the mechanics of SPRs if they are to be effectively utilised.

\subsubsection{Surface Plasmons}

A plasmon is a quasi-particle, representing the excitation of oscillations of the charges within a plasma [39-42]. In the case of SERS, the plasma is the free electrons within the metal. If an electromagnetic field is applied to the metal, it is possible to excite plasmon-polaritons, which are photons that are coupled to the charge oscillations. It is possible for the electromagnetic waves to propagate through a metal and share energy between the electromagnetic field oscillations 
and the excitation of the internal degrees of freedom of the metal. The optical response of the system in the IR and visible wavelengths are typically dominated by plasmon-polaritons for most metals.

There are two types of plasmons that exist; bulk plasmons which correspond to oscillations of the electrons within the bulk of the material, and surface plasmons [43-45] which correspond to the propagation of longitudinal charge density waves on the boundary between metals and dielectrics. Bulk plasmons can either be "pure" (exist by themselves) or exist in the form of plasmonpolaritons. Surface plasmons, on the other hand, are almost always associated with transverse electromagnetic waves and can only exist as pure plasmons in limited cases. Consequently, they are technically surface plasmon-polaritons but for simplicity I will often refer to them as surface plasmons.

\subsubsection{Localised Surface Plasmons}

In the case of SERS we are typically interested in surface plasmons that form on localised structures; for example, metallic nano-particles [46-51], roughened substrates [52,53], metallic tips [54] or specialised substrates made with nanolithography [55-57]. Furthermore, the dimensions of the structures are often at a similar order to the wavelength of the incident light. The plasmon-polaritons that form in these circumstances are called localised surface plasmon-polaritons (LSPs) and are radiative in nature; they have electric field components that exist in the far field. The most important surface plasmon mode, for SERS, has a relatively low frequency (compared to other LSP modes) and a scattered field that is the same as an electric dipole. As a result, it is often referred to as a dipolar LSP. It is also possible to have LSPs that have scattered fields that correspond to quadrupoles, octupoles or higher order multipoles.

The frequency of the LSP will be very dependent on the geometry and properties of the nano-particle [58]. If we take the simple case of a metallic sphere, the frequency of the LSP will decrease with increasing diameter and shift more to the red end of the visible spectrum. For more complicated systems, the surface plasmons have the same qualitative features but their frequencies will depend on the shape of the nano-particle.

In addition to single nano-particles, it is possible to have systems with nanoparticles that are very close together; within a couple of nanometres. In this case, the surface plasmons will interact, as the scattered field from one nanoparticle will overlap with other particles that are sufficiently close. The resulting 
surface plasmons will therefore be coupled and dependent on the strength of the interaction. In some regions, particularly in the gap between the particles, this interaction can create local electric fields that are extremely large. Using this effect, the sensitivity of SERS can reach a level where single molecules can be observed.

The LSP of a nano-particle can be excited by incident radiation that has the appropriate polarisation and frequency. As a result, there will be a resonant optical response at the LSP frequency. The resonance will have a width which will depend on the properties of the nano-particle. Because LSPs are radiative, the resonant characteristic of the system will not only be observed as a peak in the absorption by the metal, but also in the scattering coefficient. However, a large absorption coefficient does not necessarily correspond to a large electric field at a specific point on the surface of the metal (i.e. where a molecule might be) [59].

\subsubsection{The Effect of the Dielectric Function}

The local dielectric function for any material can be expressed in terms of a real and an imaginary component as:

$$
\epsilon(\omega)=\epsilon^{\prime}(\omega)+i \epsilon^{\prime \prime}(\omega),
$$

where $\epsilon^{\prime}$ is the real component and $\epsilon^{\prime \prime}$ is the imaginary component.

The real component of the dielectric function determines the resonant frequency of the LSPs. For many nano-particles, there is a maximum in the polarizability which is determined by the real component of the dielectric function. This maximum corresponds to a LSP resonance which we can tune to by adjusting the frequency of the incident radiation such that $\epsilon(\omega)$ matches the resonant conditions. The dielectric function is only dependent on the type of metal used (for example, gold or silver), however, the value of $\epsilon(\omega)$ that will cause a resonance in the polarizability will depend on the geometry of the nano-particle. A more detailed discussion on the resonant properties for different geometries will be given later in the chapter.

While the real component of the dielectric function determines the resonant frequency of the structure, the strength of the surface fields will depend largely on the imaginary component. The imaginary component determines how lossy the material is; i.e. how much of the incident light is absorbed by the metal. As a result, metals that are lossy will lose a lot of energy to optical absorption 
instead of enhancing the surface fields. The largest surface fields will, therefore, form on metals that are not very lossy in the region that a resonance occurs.

Two metals commonly used in SERS are gold $(\mathrm{Au})$ and silver $(\mathrm{Ag})$. Experimental measurements of the dielectric function have been made in references [60] and [61] and are shown in figure 3.1. Also included are fits to the real and imaginary components. For $\mathrm{Au}$, the best analytical representation of the dielectric function is $[50,61]$ :

$$
\begin{aligned}
\epsilon_{A u}(\lambda)= & \epsilon_{\infty}\left(1-\frac{1}{\lambda_{p}^{2}\left(\frac{1}{\lambda^{2}}+\frac{i}{\mu_{p} \lambda}\right)}\right) \\
& +\sum_{n=1,2} \frac{A_{n}}{\lambda_{n}}\left[\frac{e^{i \phi_{n}}}{\frac{1}{\lambda_{n}}-\frac{1}{\lambda}-\frac{i}{\mu_{n}}}+\frac{e^{-i \phi_{n}}}{\frac{1}{\lambda_{n}}+\frac{1}{\lambda}+\frac{i}{\mu_{n}}}\right],
\end{aligned}
$$

with fitting parameters: $\epsilon_{\infty}=1.54, \lambda_{p}=177.5 \mathrm{~nm}, \mu_{p}=14500 \mathrm{~nm}, A_{1}=1.27$, $\phi_{1}=-\pi / 4, \lambda_{1}=470 \mathrm{~nm}, \mu_{1}=1900 \mathrm{~nm}, A_{2}=1.10, \phi_{2}=-\pi / 4, \lambda_{2}=325 \mathrm{~nm}$ and $\mu_{2}=1060 \mathrm{~nm}$. The first term in equation 3.2 is the "Drude" contribution while the other terms are critical points in the electronic structure of Au. For $\mathrm{Ag}$, the best analytical representation of the dielectric function is [62]:

$$
\epsilon_{A g}(\lambda)=\epsilon_{\infty}\left(1-\frac{1}{\lambda_{p}^{2}\left(\frac{1}{\lambda_{2}}+\frac{i}{\mu_{p} \lambda}\right)}\right),
$$

with fitting parameters: $\epsilon_{\infty}=4, \lambda_{p}=282 \mathrm{~nm}, \mu_{p}=17000 \mathrm{~nm}$.

Focusing only on the imaginary components, $\mathrm{Ag}$ has a minimum close to $300 \mathrm{~nm}$ which then increases with wavelength. Au, on the other hand, has a relatively large $\epsilon^{\prime \prime}$ at low wavelengths with a minimum at $700 \mathrm{~nm}$. Consequently, $\mathrm{Au}$ is very lossy at low wavelengths and $\mathrm{Ag}$ is a much better option for resonances in this region. However, if the resonance frequency can be red-shifted to longer wavelengths, close to $700 \mathrm{~nm}, \mathrm{Au}$ and $\mathrm{Ag}$ become comparable.

The real components of the dielectric functions are quite similar, both are negative over the visible and IR range and decrease with increasing wavelength. Consequently, it is the imaginary component that differentiates the two metals. There are, however, additional factors that are not related to the dielectric function that may make one metal preferable over the other. For example, Au nano-particles are more stable than $\mathrm{Ag}$ as $\mathrm{Ag}$ is more susceptible to oxidation. Furthermore, Au has a higher bio-compatibility than Ag and, consequently, is 


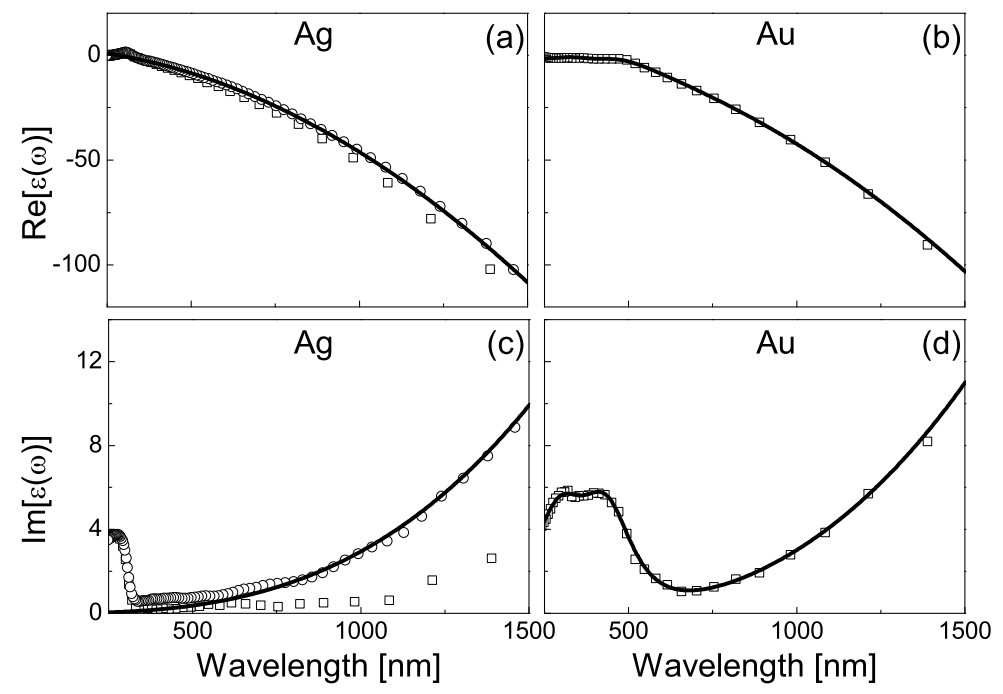

Figure 3.1: Plots of the imaginary and real components of the dielectric function for Ag and Au. Also included are fits using equations 3.2 and 3.3. The experimental data was taken from reference [60](squares) and reference [61](circles) for $\mathrm{Ag}$ and reference [60] for Au. This figure is adapted from figures E.1 and E.2 in reference [9].

widely used in biological applications.

\subsection{Raman and Fluorescence Enhancement Fac- tors}

Once the surface fields are enhanced through the LSPs, the question becomes "how does the presence of the metal affect a molecule that is placed on the surface"? There are two processes that are important in this thesis and which have been discussed in the previous chapter, Raman scattering and fluorescence. Each of these processes is affected by the presence of the metal but somewhat differently $[9,63]$.

\subsubsection{Surface Enhanced Raman Scattering}

A brief overview of the SERS enhancement factor will be given in this section. A more detailed discussion can be found in literature such as [9]. In this thesis, I will take the approach that the SERS enhancement is purely due to electromagnetic effects. However, additional chemical mechanisms $[64,65]$ may also be 
present in some cases. Raman scattering consists of two related processes, (i) excitation and (ii) emission. Each of these processes is enhanced by the LSP in the following ways.

\section{The Local Field Intensity Enhancement Factor}

Without the presence of the metallic nano-particle, the molecule will experience an electric field of $\mathbf{E}_{\text {Inc }}$. However, once the molecule is adsorbed onto the surface of a metallic nano-particle, the polarization and strength of the electric field will be modified by the LSP, and the field experienced by the molecule will become $\mathbf{E}_{\text {Loc }}\left(\omega_{L}\right)$, where $\omega_{L}$ is the frequency of the incident radiation. The local field, at the molecule location, will then induce a Raman dipole of $\mathbf{p}_{R}=\alpha_{R} \mathbf{E}_{\mathrm{Loc}}\left(\omega_{L}\right)$. The Raman dipole is, therefore, enhanced by a factor $\left|\mathrm{E}_{\mathrm{Loc}}\left(\omega_{L}\right)\right| /\left|\mathrm{E}_{\mathrm{Inc}}\right|$. However, the energy radiated by a dipole is proportional to $\left|\mathrm{p}_{R}\right|^{2}$ and, consequently, the enhancement associated with the excitation of the vibration will be given by:

$$
M_{\mathrm{Loc}}\left(\omega_{L}\right)=\frac{\left|E_{\mathrm{Loc}}\left(\omega_{L}\right)\right|^{2}}{\left|E_{\mathrm{Inc}}\right|^{2}} .
$$

This is known as the local field intensity enhancement factor.

\section{The Radiation Enhancement Factor}

Because the Raman dipole radiates in close proximity to the metal, there is a further modification to the emission spectrum. This is known as modified spontaneous emission and it affects the dipole emission in two ways. Firstly, the radiation pattern is modified. Secondly, and most importantly for SERS, the total radiated power is modified. One might expect that the dipole is unaffected by the metal and it is only the dipole radiation that is modified. However, in actuality, the emission process itself is directly affected by the metal.

This modification to the emitted radiation is dependent on the scattered frequency and, consequently, the vibrational modes will be modified differently. This enhancement is called the radiative enhancement factor, $M_{\text {Rad }}^{d}\left(\omega_{R}\right)\left(\omega_{R}\right.$ is the frequency of the scattered light), where the superscript $d$ refers to the direction in which the radiation is being detected. To a good approximation, the radiative enhancement can be assumed to be the same as the local field enhancement with a dependence on the scattered frequency $[66,67]$ : 


$$
M_{\text {Rad }}^{d}\left(\omega_{R}\right) \approx \frac{\left|E_{\mathrm{Loc}}\left(\omega_{R}\right)\right|^{2}}{\left|E_{\mathrm{Inc}}\right|^{2}} .
$$

The scattered light can be either Stokes- or anti-Stokes-shifted from the incident wavelength.

\section{The SERS enhancement factor}

The overall single molecule enhancement to the Raman signal of a vibrational mode with frequency $\omega_{\nu}=\omega_{L}-\omega_{R}$ is:

$$
M_{\mathrm{SERS}}=M_{\mathrm{Loc}}\left(\omega_{L}\right) M_{\mathrm{Rad}}^{d}\left(\omega_{R}\right) .
$$

Using the approximation in equation 3.5 we can rewrite this as:

$$
M_{\mathrm{SERS}}\left(\omega_{L}, \omega_{R}\right) \approx M_{\mathrm{Loc}}\left(\omega_{L}\right) M_{\mathrm{Loc}}\left(\omega_{R}\right)=\frac{\left|E_{\mathrm{Loc}}\left(\omega_{L}\right)\right|^{2}}{\left|E_{\mathrm{Inc}}\right|^{2}} \frac{\left|E_{\mathrm{Loc}}\left(\omega_{R}\right)\right|^{2}}{\left|E_{\mathrm{Inc}}\right|^{2}},
$$

which can be simplified for negligible Raman shifts to:

$$
M_{\mathrm{SERS}}\left(\omega_{L}\right) \approx \frac{\left|E_{\mathrm{Loc}}\left(\omega_{L}\right)\right|^{4}}{\left|E_{\mathrm{Inc}}\right|^{4}} .
$$

This approximation is valid to within an order of magnitude for many LSP modes and vibrational states. Equation 3.8 is often referred to as the $|E|^{4}$ approximation in the literature [68]. The Stokes or anti-Stokes cross-section is now given by:

$$
\frac{d \sigma_{\mathrm{SERS}}\left(\omega_{R}\right)}{d \Omega} \approx M_{\mathrm{SERS}}\left(\omega_{L}\right) \frac{d \sigma_{\mathrm{Raman}}\left(\omega_{R}\right)}{d \Omega} .
$$

\subsubsection{Surface Enhanced Fluorescence}

As in the previous section, only a brief introduction to surface enhanced fluorescence (SEF) will be given and the reader is directed to literature such as [9] for a more detailed overview of the technique. Surface enhanced fluorescence is very similar to SERS in that it consists of both an excitation of the molecule followed by the emission of a photon which is shifted from the wavelength of the incident radiation [69-72]. The key difference in the two processes is that the two events are instantaneous for Raman scattering but there is a delay for fluorescence. As a result, for fluorescence, the two steps occur independent of 
each other.

\section{The Absorption Enhancement}

The first step in the fluorescence process is the absorption of an incident photon and the excitation of the molecule to the first electronic state. As in Raman scattering, the absorbed power is proportional to the square of the electric field. The enhancement in absorption is therefore just the local field intensity enhancement factor discussed previously in section 3.2.1 and given in equation 3.4. The surface modified absorption cross-section is therefore given by a factor:

$$
\sigma_{\mathrm{Abs}}^{M}=M_{\mathrm{Loc}}\left(\omega_{L}\right) \sigma_{\mathrm{Abs}},
$$

where $\sigma_{\mathrm{Abs}}$ is the unmodified absorption cross-section.

\section{Modified Fluorescence}

Because there is a delay from when a photon is absorbed and a fluorescence photon is emitted, it is impossible to enhance the fluorescence cross-section such that it is greater than the modified absorption cross-section shown in equation 3.10. There will, however, be a modification to the decay rates of the various relaxation processes. The two processes that are available under unmodified conditions, fluorescence decay $\left(\Gamma_{\mathrm{Rad}}^{M}\right)$ and intrinsic non-radiative decay $\left(\Gamma_{\mathrm{NR}}^{0}\right)$ (see section 2.4), are still present, but the radiative decay rate is enhanced by a factor $M_{\text {Rad }}$ so that $\Gamma_{\mathrm{Rad}}^{M}=M_{\mathrm{Rad}} \Gamma_{\mathrm{Rad}}$ ( $\Gamma_{\mathrm{Rad}}$ is the unmodified radiative decay rate). This is the radiative enhancement factor discussed in section 3.2.1 but averaged over all scattering wavelengths and directions. The reason that we have the same enhancement factor as for Raman scattering is because fluorescence is similarly affected by modified spontaneous emission. However, in the case of fluorescence, it is the decay rate that is modified, while for Raman scattering it is the radiated power that is modified. Unlike Raman scattering, fluorescence occurs over a continuous range of wavelengths that can be over $100 \mathrm{~nm}$ in width. As a result, the LSP resonance will tend to favour certain fluorescence frequencies more than others resulting in a modified fluorescence spectral shape [73].

In contrast to fluorescence decay, the intrinsic Non-Radiative decay rate is unmodified by the presence of the metal. This is because $\Gamma_{\mathrm{NR}}^{0}$ is determined by interactions between states within the molecule and interactions with the 
surrounding molecules (not including the metal).

A new decay process becomes available in the surface modified case, nonradiative emission, $\Gamma_{\mathrm{NR}}^{\mathrm{EM}}$. A fraction of the radiated light will be absorbed by the metallic nano-particle and, consequently, not observed in the far field. It is therefore a non-radiative process $[9,70]$ which enhances the unmodified radiative decay rate as there is an initial emission by the molecule before the radiation is absorbed by the metal. Hence, the non-radiative emission decay rate is given by $\Gamma_{\mathrm{NR}}^{\mathrm{EM}}=M_{\mathrm{NR}} \Gamma_{\mathrm{Rad}}$, where $M_{\mathrm{NR}}$ is the non-radiative enhancement factor. While the local and radiative enhancement factors are related to the electric field strength, the non-radiative enhancement factor is mostly dependent on the distance between the molecule and the metal. For a dipole close to a metallic surface, $M_{\mathrm{NR}}$ is approximately given by [74]:

$$
M_{\mathrm{NR}} \approx 1+\frac{3 \kappa}{8\left(k_{M} d\right)^{3}} \operatorname{Im}\left(\frac{\epsilon-\epsilon_{M}}{\epsilon+\epsilon_{M}}\right),
$$

where $k_{M}=(\omega / c) \sqrt{\epsilon_{M}}$ ( $\omega$ is the excitation frequency and $\epsilon_{M}$ is the dielectric constant of the medium), $d$ is the distance between the dipole and the surface, $\kappa$ can vary between 1 (dipole parallel to surface) and 2 (dipole perpendicular to surface), and $\epsilon$ is the dielectric function of the metal.

It is important to note that, as in the case of the SERS, the presence of the metal directly affects the emission properties of the molecule itself. However, it is the decay rates that are modified under fluorescence as the number of scattered photons must be equal to or less than the number of absorbed photons. This is not the case for Raman as the absorption and emission is instantaneous and cannot be de-coupled. One might expect that the emission decay rate is unaffected by the metal and it is only the fact that some of the radiated photons must pass through the metal that creates this new decay process. However, as with the radiative enhancement factor, the total emission rate (non-radiative + radiative) from the molecule is directly modified by the presence of the metal. This is a well known result from electromagnetic theory in which a dipole is radiating close to a plane metal surface [9].

Using these decay rates, we can express the surface modified quantum yield as:

$$
Q^{M}=\frac{\Gamma_{\mathrm{Rad}}^{M}}{\Gamma_{\mathrm{Rad}}^{M}+\Gamma_{\mathrm{NR}}^{E M}+\Gamma_{\mathrm{NR}}^{0}},
$$

which can be written in terms of the unmodified decay rates, enhancement 
factors and the unmodifed quantum yield (see equation 2.17) as:

$$
\begin{aligned}
Q^{M} & =\frac{M_{\mathrm{Rad}} \Gamma_{\mathrm{Rad}}}{M_{\mathrm{Tot}}\left(\omega_{S}\right) \Gamma_{\mathrm{Rad}}+\Gamma_{\mathrm{NR}}^{0}} \\
& =\frac{M_{\mathrm{Rad}}}{M_{\mathrm{Tot}}+\left(Q^{0}\right)^{-1}-1}
\end{aligned}
$$

where $M_{\text {Tot }}$ is the total emission enhancement factor and defined as the sum of the non-radiative and radiative enhancement factors, $M_{\mathrm{Tot}}=M_{\mathrm{Rad}}+M_{\mathrm{NR}}$. If $\left(Q^{0}\right)^{-1}<<M_{\text {Tot }}$ then this equation can be simplified to:

$$
Q^{M}=\frac{M_{\mathrm{Rad}}}{M_{\mathrm{Tot}}} .
$$

The fluorescence cross-section for a specific scattering wavelength and under surface-enhanced conditions can now be given as:

$$
\frac{d \sigma_{\mathrm{SEF}}\left(\omega_{S}\right)}{d \Omega} \approx \frac{M_{\mathrm{Rad}}^{d}\left(\omega_{S}\right)}{M_{\mathrm{Tot}}} \sigma_{\mathrm{Abs}}^{M}=\frac{M_{\mathrm{Rad}}^{d}\left(\omega_{S}\right)}{M_{\mathrm{Tot}}} M_{\mathrm{Loc}}\left(\omega_{L}\right) \sigma_{\mathrm{Abs}}
$$

It is important to note that $\sigma_{\mathrm{SEF}}$ is dependent on $M_{\mathrm{Rad}}^{d}\left(\omega_{S}\right)$ as we are observing the scattered light at a specific direction and frequency. Comparing this with equation 2.19 from the previous chapter we can obtain a fluorescence enhancement factor of:

$$
M_{\mathrm{SEF}} \approx \frac{M_{\mathrm{Loc}}\left(\omega_{L}\right) M_{\mathrm{Rad}}^{d}\left(\omega_{S}\right)}{Q^{0} M_{\mathrm{Tot}}} .
$$

Despite the fact that we refer to fluorescence close to a metallic surface as "surface-enhanced fluorescence", it is possible to have an enhancement factor that is less than one, resulting in a quenched fluorescence signal. This will occur if $Q^{0} M_{\text {Tot }}>M_{\text {Loc }}\left(\omega_{L}\right) M_{\text {Rad }}\left(\omega_{S}\right)$ which may happen if non-radiative emission is substantially larger than radiative emission. This will typically occur when molecules are adsorbed very close to the metallic surface. Consequently, SEF is often performed on molecules that are suspended several nanometers from the surface [75-77]. Although many aspects of this model have been measured experimentally $[70,73,74,78]$, reliable measurements of the total decay rate for molecules directly adsorbed on metallic surfaces are yet to be achieved. I will return to this problem in a later chapter. 


\subsection{Examples of SERS Enhancements}

There are several basic nano-particle geometries that are very important for SERS and SEF, all of which are based on the basic metallic sphere. The experiments carried out in this thesis consist of substrates formed by aggregates of Ag colloids which can be modelled closely by metallic spheres. The aim of this section is, therefore, to give a basic overview of the enhancement properties of these systems.

\subsubsection{The Metallic Sphere}

A metallic sphere isolated within a dielectric medium is a relatively simple system that has been investigated in great detail. The simplest theory for the enhancement properties of a metallic sphere is obtained with an electrostatic approximation of the incident field. However, in order to understand the enhancement factor distribution for real systems, a full Mie theory treatment is required.

\section{Electrostatic Approximation Solution}

Within the electrostatic approximation, it can be shown that the dipolar polarizability of the sphere is given by [9]:

$$
\alpha_{E S}=4 \pi \epsilon_{0} \epsilon_{M} a^{3} \beta_{S}(\omega)
$$

where:

$$
\beta_{S}(\omega)=\frac{\epsilon(\omega)-\epsilon_{M}}{\epsilon(\omega)+2 \epsilon_{M}}
$$

$a$ is the diameter of the sphere, $\epsilon_{M}$ is the dielectric constant of the medium, and $\epsilon(\omega)$ is the dielectric function of the metal. For the electrostatic case, the system will have a resonance when the real component of the dielectric function is $\epsilon(\omega)=-2 \epsilon_{M}$ (due to the denominator in equation 3.18). Furthermore, the resonance frequency is independent of the dimensions of the sphere and only varies with the properties of the metal itself. It is now possible to write the absorption and scattering coefficients as:

$$
Q_{\mathrm{Abs}}=\frac{\sigma_{\mathrm{Abs}}}{\sigma_{\mathrm{Geom}}}=4 k_{M} a \operatorname{Im}\left(\beta_{S}\right)
$$



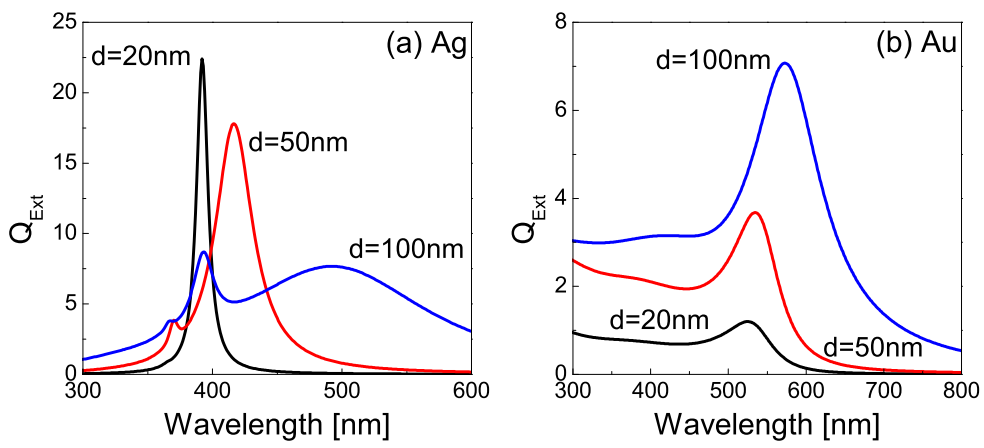

Figure 3.2: $\quad$ Plots of the wavelength dependence of the extinction coefficient for an (a) $\mathrm{Ag}$ and (b) Au metallic sphere immersed in water with diameters: $20 \mathrm{~nm}, 50 \mathrm{~nm}$ and $100 \mathrm{~nm}$. The calculations were performed using Mie theory. This figure was adapted from figure 6.4 in reference [9].

$$
Q_{\mathrm{Sca}}=\frac{\sigma_{\mathrm{Sca}}}{\sigma_{\mathrm{Geom}}}=\frac{8}{3}\left(k_{M} a\right)^{4}\left|\beta_{S}\right|^{2},
$$

where $\sigma_{\text {Geom }}$ is the actual geometrical cross-section of the sphere. There are however, a couple of problems associated with the electrostatic approximation solution:

- Energy conservation is not fulfilled. There have been several proposals [79, 80] for a modification to $\beta_{S}$ that will conserve energy but it is uncertain how useful they are.

- The fact that the polarizability is scale invariant does not agree with experiment, especially at dimensions that approach the wavelength of the incident radiation.

Consequently, this approximation is only valid if the dimensions of the nanoparticle are much smaller than the wavelength of the incident radiation.

\section{Mie Theory Solution}

With a structure as simple as a metallic sphere, an analytical solution can be determined with a complete electromagnetic treatment. To avoid unnecessary details, only the results of Mie theory calculations are given. A more comprehensive overview can be found in literature such as [9]. Because LSPs affect both the absorbed and scattered energy, the extinction coefficient, $Q_{\text {Ext }}$, is sufficient for revealing the resonance nature of the sphere. Figure 3.2 shows the 


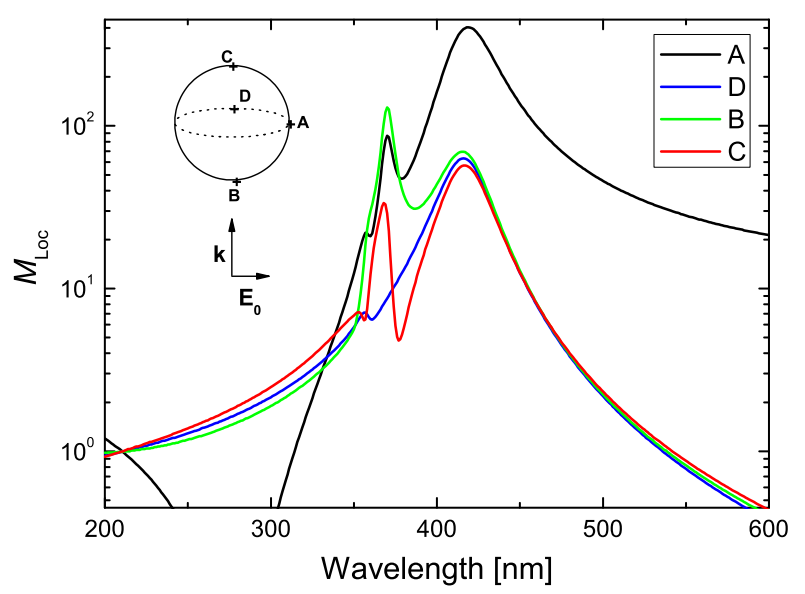

Figure 3.3: $\quad$ The wavelength dependence of the local field enhancement factor at four different locations on a Ag sphere, in water, with a diameter of $50 \mathrm{~nm}$. The y-axis is logarithmic while the $x$-axis is linear. Also included is a figure showing the position of the points on the sphere, and the incident angle and polarization of the excitation radiation. This figure was adapted from figure 6.8 in reference [9].

distribution of the extinction coefficient with the wavelength of the incident radiation for a metallic sphere $(\mathrm{Ag}$ and $\mathrm{Au})$ in water, and three different sphere diameters.

For both $\mathrm{Ag}$ and $\mathrm{Au}$, the resonance shifts to longer wavelengths as the diameter increases. This is due to retardation effects. Furthermore, the resonance is shifted more to the red end of the visible spectrum for Au than Ag. This is fortunate as $\mathrm{Au}$ is not as lossy at longer wavelengths. However, it is still short of the region where Au becomes comparable to silver (in terms of absorption) and, consequently, the local field enhancement will not be as strong as Ag.

Figure 3.3 shows the local enhancement, $M_{\text {Loc }}$, at four different locations on a silver sphere with a diameter of $50 \mathrm{~nm}$, in water. For most positions, there are two major resonances and one minor resonance, each of which occur at a similar wavelength for the different locations. The longest wavelength resonance ( $\sim 420 \mathrm{~nm}$ ) corresponds to a dipolar LSP whilst the shorter wavelength resonance $(\sim 370 \mathrm{~nm})$ corresponds to a quadrupolar LSP. At larger diameters, the wavelength that corresponds to the largest local field may vary slightly depending on the surface location being observed [9] .

The magnitude of the local field enhancement, for any sphere diameter, will vary with the position on the surface. The largest electric fields will typically form in regions where the surface is perpendicular to the incident field polariza- 
tion (point A). One might assume that the local field at points B (at the front of the nano-particle) and $\mathrm{C}$ (at the back) would have the same distribution. While they do have the same general shape, due to the k-vector of the incident light point $B$ receives a larger enhancement. In fact, the maximum enhancement for the resonance at $\sim 370 \mathrm{~nm}$ occurs at point B. Finally, point D, where the field is parallel to the surface, has a similar enhancement factor distribution to B and $\mathrm{C}$ but without the large resonance at low wavelengths. This is not surprising as the incident field is also parallel to the surface at point $\mathrm{D}$.

\subsubsection{The Metallic Dimer}

The SERS enhancement factors that can be achieved with a metallic sphere are relatively low, ranging from $10^{5}-10^{6}[9]$. In order to get the large enhancements that are necessary for single molecule measurements, we need enhancement factors closer to $10^{9}-10^{10}$. One of the most effective ways to obtain these large enhancements is to aggregate metallic nano-particles. The LSPs will interact with each other and create extremely large electric fields in the regions in between the particles; called hot-spots. The simplest system of aggregated particles is the metallic dimer which consists of two symmetric metallic spheres of diameter $a$ and separated by a distance $d$. While this system is much more complicated than a single metallic sphere, a numerical solution can be found using generalized Mie Theory (GMT) [81]. As in the previous section, I will not go into the detail of the theory, but give the results and refer the reader to literature such as [9] for a more detailed overview of the topic.

\section{Gap Dependency}

A determination of the resonance wavelength can be made from the wavelength dependence in the extinction coefficient. For the case of a dimer, the resonance wavelength will not only vary with the particle diameter, as it does for a single sphere, but also the gap size. The distribution in the extinction coefficient with wavelength for a $50 \mathrm{~nm}$ diameter $\mathrm{Ag}$ and $\mathrm{Au}$ dimer for several different gap sizes are shown in figure 3.4. In these simulations, the polarization of the incident radiation is set parallel to the dimer axis as to efficiently couple to the LSPs that enhance the electric field in the gap region. Also included is the extinction coefficient spectrum for a single $50 \mathrm{~nm}$ sphere. For both metals, the main resonance shifts to longer wavelengths as the nano-particles get closer together. In addition, there are several other resonances that appear as the 

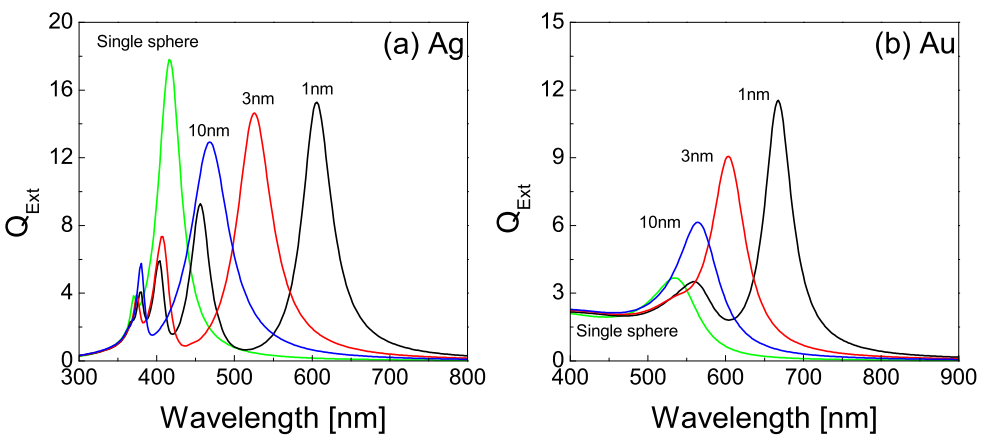

Figure 3.4: Plots of the wavelength dependence of the extinction coefficient for an (a) Ag dimer and an (b) Au dimer. The diameter of the spheres are $50 \mathrm{~nm}$ with gap sizes $1 \mathrm{~nm}, 3 \mathrm{~nm}$ and $10 \mathrm{~nm}$. Also included is the single sphere extinction coefficient spectrum for both Ag and Au but magnified by a factor of two. This figure was adapted from figure 6.19 and 6.20 in reference [9].

gap size decreases. The longest wavelength resonance corresponds to dipolar coupling between LSPs that have dipole polarizations along the gap axis (see figure 3.5). On the other hand, the shorter wavelength resonances correspond to dipolar coupling of the higher order single-sphere LSP resonance (for example, quadrupolar LSPs). These resonances are more numerous in the case of Ag due to its intrinsic lower absorption.

For the case of $\mathrm{Au}$, the resonance location can be red-shifted close to $\sim$ $700 \mathrm{~nm}$ which is in the region where $\mathrm{Au}$ is not particularly lossy (relative to $\mathrm{Ag}$ at least). Consequently, the local field enhancement for $\mathrm{Au}$ dimers with gap sizes close to $\sim 1 \mathrm{~nm}$ should be comparable to that which can be achieved with a Ag dimer. Smaller gap sizes may not be physical as SERS analytes, which need to fit in the gap, often have widths around this size. Furthermore, generalized Mie theory may no longer be accurate for gap sizes smaller than $1 \mathrm{~nm}$.

Often the suitability of a particular substrate for SERS applications lies not in the location of the resonance, but in the size of the local field enhancement. Figure 3.6 shows the SERS enhancement factor on the surface of one of the spheres at the gap location for $\mathrm{Ag}$ and $\mathrm{Au}$, and several gap sizes. As the gap size decreases the SERS enhancement factor increases and the maximum is shifted to longer wavelengths. This is not surprising as the coupling strength of the LSPs will depend on how close they are and, as we have seen from the extinction coefficient, the resonance shifts more to the red end of the visible spectrum as the gap size decreases. The presence of the shorter wavelength resonances is 
Figure 3.5: A schematic of a metallic dimer with the direction of incident light $\mathbf{k}$, and polarization, $\mathbf{E}_{\text {Inc. Also }}$ shown is the polarization of the LSPs when the excitation wavelength matches the red-shifted resonance. At other excitation frequencies, the LSP polarization will be similar (for this incident polarization) but with a smaller amplitude as the incident light cannot couple to the LSPs that are perpendicular to the gap axis.

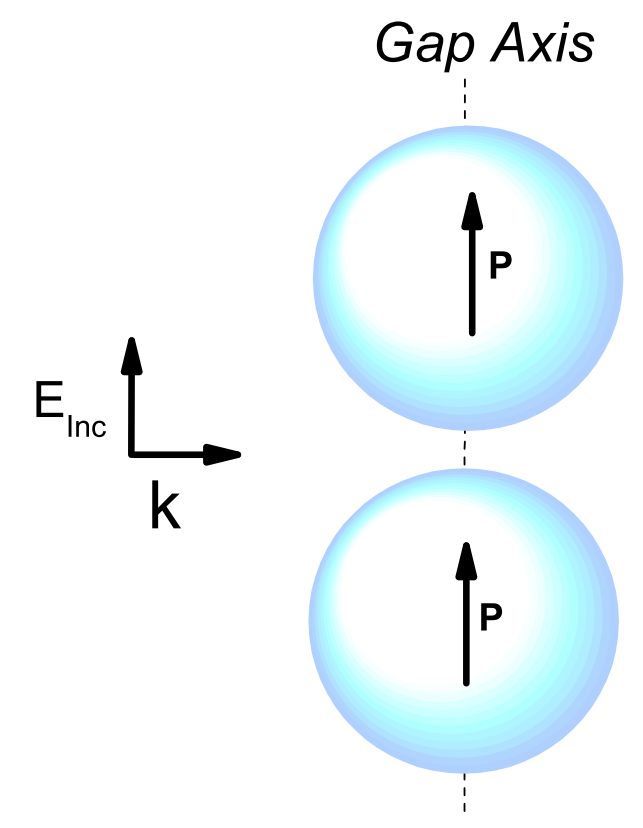

also apparent, especially in the case of Ag. Also included in the plots is the average enhancement over the whole surface, for a gap size of $2 \mathrm{~nm}$. For both metals, the gap SERS enhancement is more than two orders of magnitude larger than the average, demonstrating the sensitivity of these systems to molecules located in the gap regions.

For both $\mathrm{Ag}$ and $\mathrm{Au}$, the maximum SERS enhancement that can be achieved is $\sim 10^{11}$ which is $5-6$ orders of magnitude larger than what is achievable with a single $\mathrm{Ag}$ sphere. For the case of Au, the advantage of LSP coupling is particularly apparent as the SERS enhancement is eight orders of magnitude larger than a single Au sphere. This is due to the red-shifting of the resonance wavelength to a region where the metal is not so lossy. However, if gap sizes this small cannot be obtained in practice, Ag will typically be the best choice of metal.

Using an electromagnetic package called FemLab ${ }^{\circledR}[82]$ it is possible to simulate the electromagnetic field in the region between the metallic spheres. Figure 3.7 shows the distribution of SERS enhancements, within the electrostatic approximation, in the gap region for a Au dimer in air with parameters $a=60 \mathrm{~nm}$ and $d=2 \mathrm{~nm}$ and an excitation wavelength of $559 \mathrm{~nm}$ (the main coupled-LSP resonance wavelength). The maximum SERS enhancement for this system is $10^{8}$. However, this greatly depends on the location of the molecule. If the 

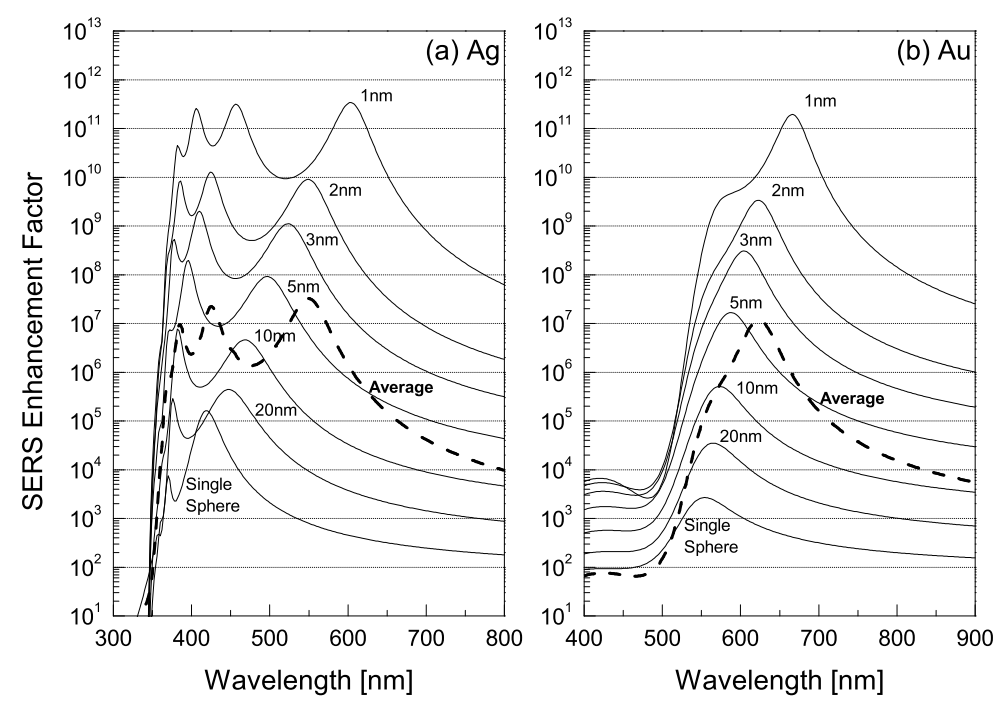

Figure 3.6: Plots of the wavelength dependence of the SERS enhancement factor on the surface of the metal and in the centre of the gap for both an (a) Ag dimer and an (b) Au dimer. The polarization of the incident light is aligned with the axis of the gap as in figure 3.5. The diameter of the spheres is $50 \mathrm{~nm}$ and the enhancement factor spectrum is calculated for several gap sizes. Also included is the SERS enhancement factor on the surface of a single sphere (of the same diameter) at the location where the surface is perpendicular to the incident electric field. Furthermore, the average SERS enhancement factor integrated over the whole surface, for the $2 \mathrm{~nm}$ gap dimer, is shown (the bold dashed curve). This figure is taken from figures 6.19 and 6.20 in reference [9]. 


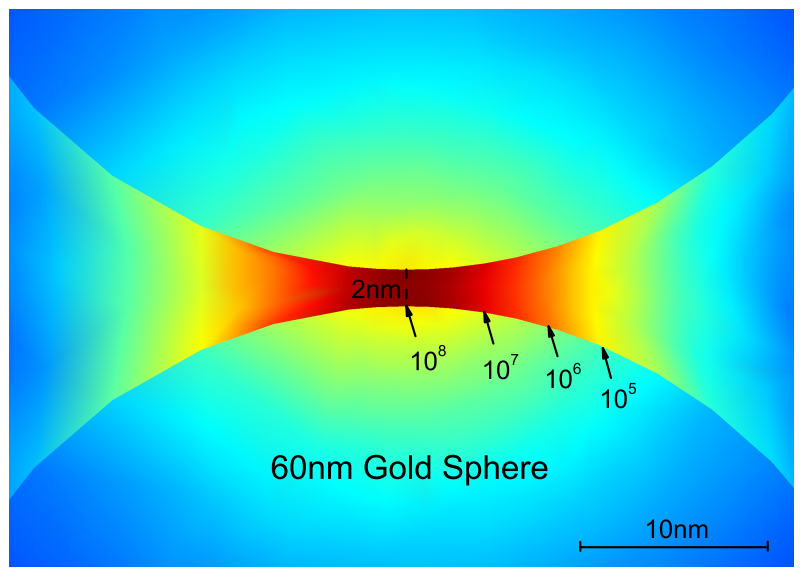

Figure 3.7: A simulation of the SERS enhancement in the gap region of a gold dimer in air with dimensions of $a=60 \mathrm{~nm}$ and $d=2 \mathrm{~nm}$. The simulation is three dimensional but only a cross-section going through the centre of the spheres is shown. Furthermore, the simulation is performed with an electrostatic approximation. The colour corresponds to the magnitude of the SERS enhancement on a log scale with red corresponding to an extremely large enhancement $\sim 10^{8}$ and blue corresponding to a small enhancement $\sim 10^{3}$.

molecule moves away from the gap by only $3 \mathrm{~nm}$, the SERS enhancement will decrease by an order of magnitude. Consequently, the SERS enhancement for these systems are very sensitive to the location of the molecule.

\section{Polarization Effects}

For a metallic sphere, the size of the local field enhancement and wavelength of the resonance are independent of the polarization of the incident radiation. Only the surface position that corresponds to maximum enhancement will change. This is not the case for a metallic dimer. The largest enhancements will typically occur at the gap location, independent of the incident polarization [83,84]. However, the strength of the coupled-LSP will vary with polarization [85]. Figure 3.8 shows the wavelength dependence of the local field enhancement factor at the gap location for an $\mathrm{Ag}$ dimer in air, with a diameter of $50 \mathrm{~nm}$ and a gap size of $2 \mathrm{~nm}$. Three different incident angles and two different polarizations $\left(E_{p}\right.$ and $E_{s}$ ) are taken as shown in figure 3.8(a).

The red-shifted resonance corresponding to the coupled-LSP shown in figure 3.5 can only be observed if the incident light has an electric field component along the gap axis. This occurs for two of the six cases; $E_{p}$ in figures 3.8(b) 


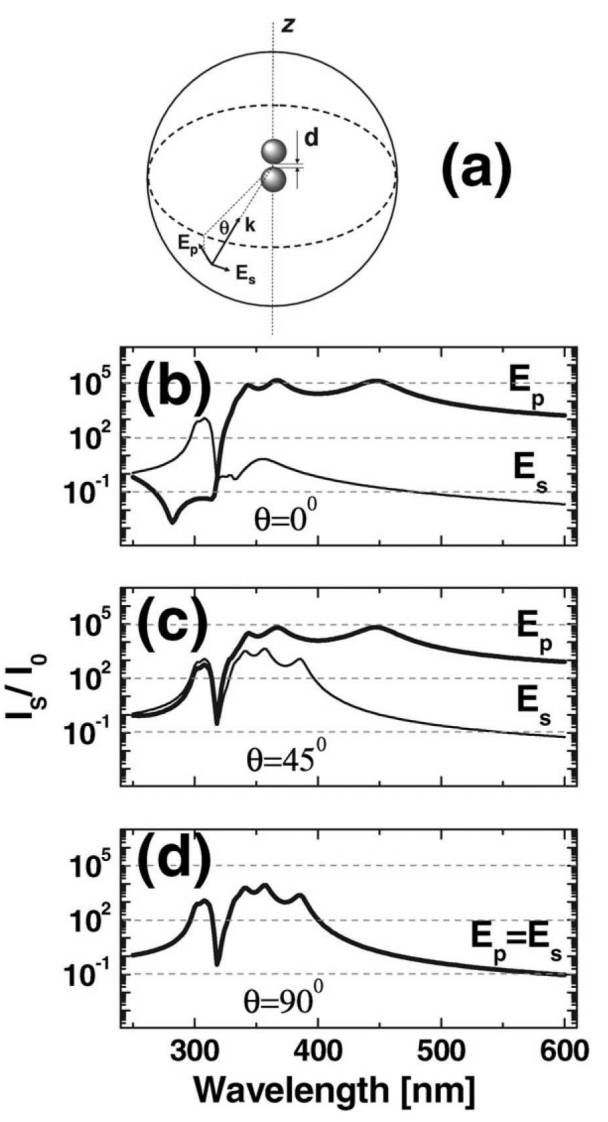

Figure 3.8: The wavelength dependence of the local field enhancement factor at the surface and in the centre of the gap for different polarizations and incident angles. (a) shows a schematic of the incident angle and the two polarizations. The local field enhancement is expressed as the ratio of the surface intensity to the field intensity if the spheres were not there, which are equivalent (see equation 3.4). The incident angle is taken at (b) $0^{\circ}$, (c) $45^{\circ}$, and (d) $90^{\circ}$. This figure was taken from figure 1 in reference [85]. 


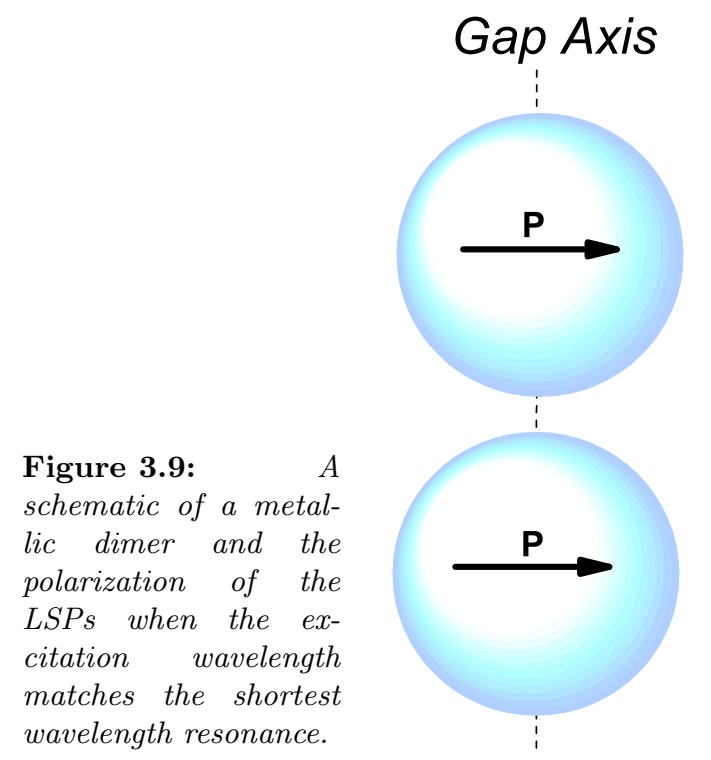

and (c). For these two cases, the maximum local field enhancement is very similar and most of the resonance wavelengths are the same. There is, however, an additional resonance when the incident light has an angle of $\theta=45^{\circ}$ close to $310 \mathrm{~nm}$ which corresponds to a single-sphere dipolar LSP. The LSP in this case has a polarization that is perpendicular to the gap axis (see figure 3.9) and, hence, the coupling strength between spheres is very weak. The system therefore acts as a single sphere at this wavelength for certain polarizations (all cases apart from $E_{p}$ in figure 3.8(b)). It is possible to excite this LSP mode if the incident light has an electric field component that is perpendicular to the gap axis. There are also several medium wavelength resonances between $320 \mathrm{~nm}$ and $420 \mathrm{~nm}$ which correspond to higher order coupled-LSPs. These resonances are very case dependent and have resonant wavelengths that vary with polarization. Furthermore, some of these resonances will only be observable for particular polarizations and incident angles.

An interesting feature of these systems is the relationship between the incident polarization and the polarization of the electric field in the gap region. Figure 3.10 shows the ratio of the z-component of the electric field intensity and the total intensity in the gap for the three incident angles and only an $E_{p}$ polarization. We have taken the z-component because the gap axis is the z-axis. For incident angles of $\theta=0^{\circ}$ and $45^{\circ}$, the gap field is mostly along the z-direction for incident wavelengths above $320 \mathrm{~nm}$; apart for some minor perturbations cor- 


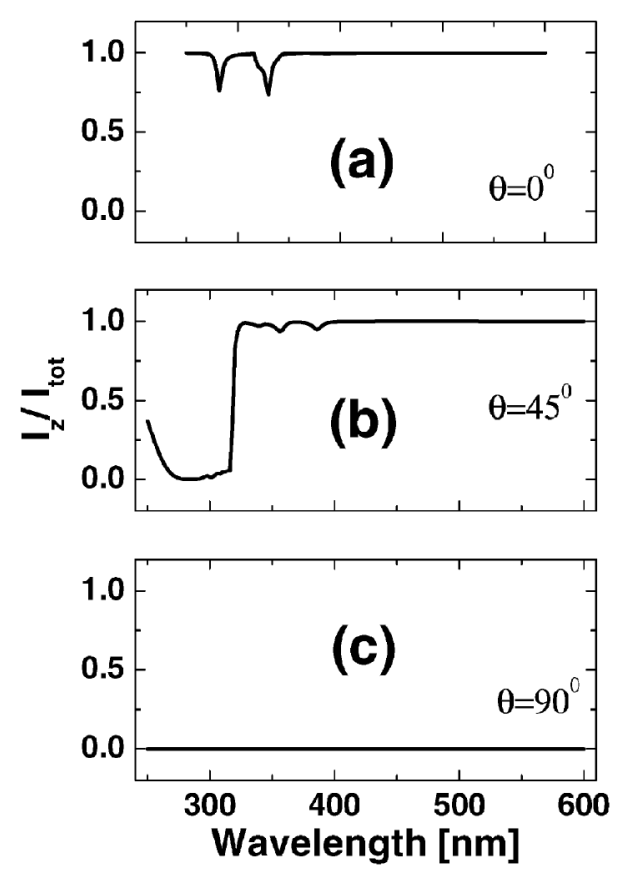

Figure 3.10: Plots of the ratio of the field intensity along the gap axis (the $z$ axis) and the total intensity in the gap of the $\mathrm{Ag}$ dimer shown in figure 3.8 . The ratio is plotted as a function of wavelength. The polarization is along $E_{p}$ for all cases and the incident angle is taken at (a) $0^{\circ}$, (b) $45^{\circ}$, and (c) $90^{\circ}$. This figure was taken from figure $2 \mathrm{in}$ reference [85].

responding to higher order LSP couplings. For the red-shifted resonance, all of the gap field is polarized along the gap axis. Unsurprisingly, the gap field has no component along the gap axis for an incident angle of $\theta=90^{\circ}$ as the incident light also has no component along that direction.

For SERS applications we are typically interested in the red-shifted coupledLSPs as these correspond to the largest local field enhancements. In these cases, the polarization of the incident light does not matter as long as there is a component along the gap axis. Furthermore, the electric field that a molecule in the gap experiences will always be along the gap axis at sufficiently long wavelengths.

\subsubsection{Large Colloidal Aggregates}

It is rarely the case that a single metallic dimer is observed. All of the experiments performed in this thesis consist of SERS measurements performed on Ag colloidal aggregates that can consist of many nano-particles (see figure 3.11). As a result, we do not observe a single hot-spot that has formed between two spheres but many hot-spots, all with comparable local field enhancements. For these systems, the hot-spots will tend to form in the gap between the nano- 

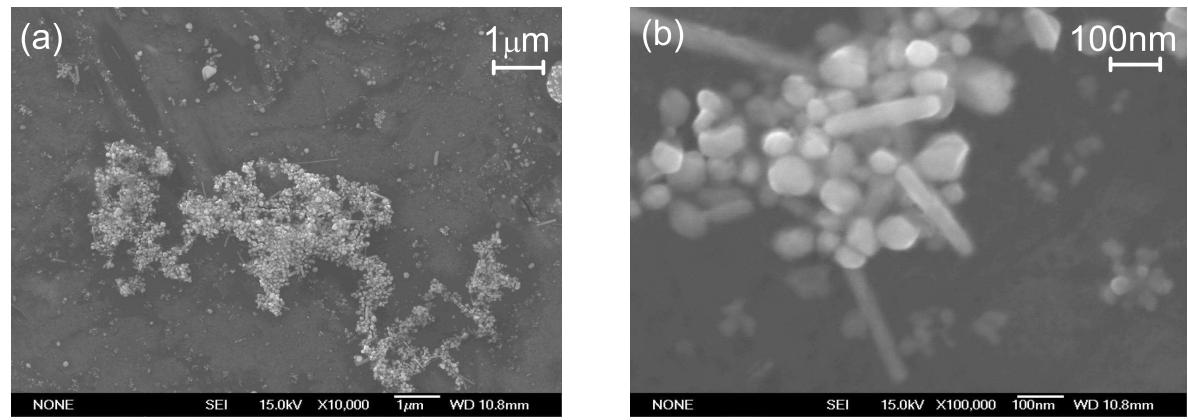

Figure 3.11: Scanning Electron Microscopy (SEM) images of an aggregate of $\mathrm{Ag}$ nano-particles. (b) is a close up of a region of the image in (a). The scale of the images are shown at the top right of each figure.

particles for reasons discussed in the previous section. However, the resonance wavelength will vary due to gap and nano-particle sizes. The distribution of hot-spots will therefore be dependent on the excitation wavelength.

\section{The Dispersion in the Enhancement Factor Distribution}

Figure 3.12 shows a simple two dimensional simulation (performed using FemLab ${ }^{\circledR}$ ) consisting of a series of randomly positioned Au nano-particles which have the same diameter $(60 \mathrm{~nm})$. Because the particles sizes are all the same, one of the variables that modifies the resonant wavelength is lost, but it will still be modified by the gap size. The simulations were performed with five different excitation wavelengths.

Irrespective of which wavelength is used, hot-spots will form in a few of the gap regions. However, as the wavelength is varied, the location of the hot-spots changes. The distribution of hot-spots is therefore dependent on the incident wavelength. If we consider a SERS experiment performed on this system, with the SERS analyte uniformly adsorbed onto the surface of the nano-particles, the molecules that largely contribute to the SERS signal at one wavelength may contribute very little at another wavelength.

Compared to substrates that are created with techniques such as nanolithography, these systems have the disadvantage that we have very little control over the LSPs. However, it is not currently possible to obtain the local field enhancement factors achievable with these structures using more customised substrates. This is very important for applications such as single molecule SERS. 

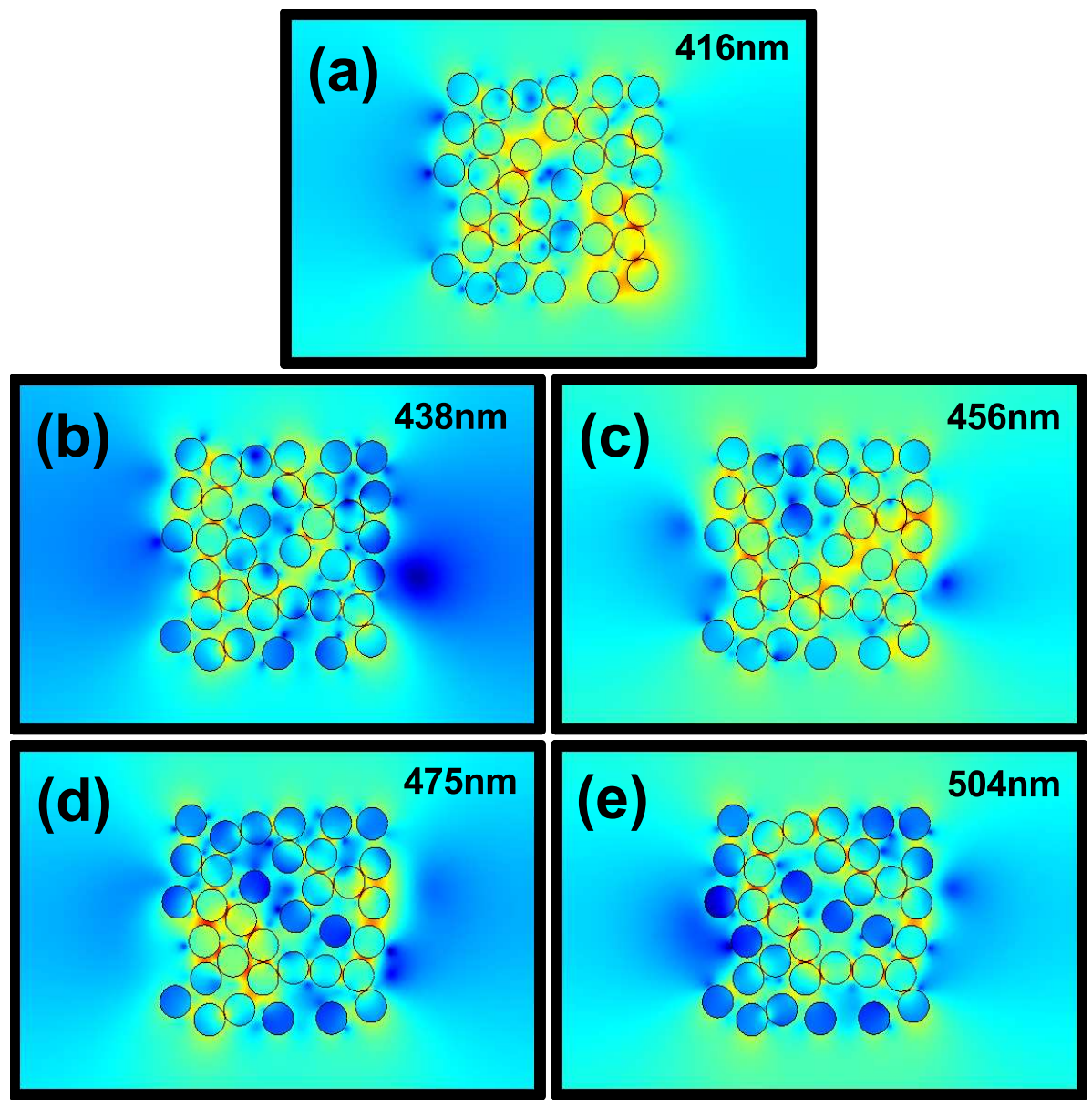

Figure 3.12: A two dimensional simulation of the SERS enhancement for an array of 35 Au nano-particles with $60 \mathrm{~nm}$ diameters and randomly positioned. The incident field is electrostatic and along the vertical axis but the dielectric function of the particles are the same as Au. The colour corresponds to the magnitude of the local enhancement on a log scale with red corresponding to an extremely large enhancement and blue corresponding to a small enhancement. A simulation is performed for five different excitation wavelengths: (a) $416 \mathrm{~nm}$, (b) $438 \mathrm{~nm}$, (c) $456 \mathrm{~nm}$, (d) $475 \mathrm{~nm}$ and (e) $504 \mathrm{~nm}$. 


\section{The Average Enhancement Factor and Long-Tail Statistics}

It is useful at this point to discuss the interpretation of the enhancement factor that we measure in SERS. Typically, many molecules contribute to the observed SERS signal. The fraction of the overall signal that each molecule contributes will be proportional to the SERS enhancement factor it experiences. However, the local enhancement is extremely sensitive to the local environment and, consequently, will vary dramatically. It is therefore possible to define a single molecule enhancement factor (SMEF) which is dependent on the location of the molecule within the system and calculated using equation 3.8. Furthermore, we can define an average enhancement factor (AEF) which is the average SMEF for all molecules contributing to the signal. Typically we will measure the AEF unless single molecule measurements are performed.

In section 3.3.2, it was shown that the local enhancement factor decreases very quickly as the molecule moves away from the centre of the gap. The area of the colloid that is considered part of the hot-spot is therefore very small $\sim 4 \mathrm{~nm}^{2}$. For a sphere of diameter $50 \mathrm{~nm}$, the fraction of the molecules that are in the hot-spot will be $\sim 1 / 1000$. As a result, only an extremely small fraction of the molecules adsorbed on the surface (assuming a uniform distribution) will experience the largest enhancements. Studies have shown that for many common SERS substrates (like the ones used in this thesis), the distribution of enhancement factors follows a long-tail distribution with very few molecules experiencing extremely large enhancement factors and many molecules experiencing relatively small enhancement factors [86,87]. For such systems, it takes an extremely large number of events for the average to converge which makes SERS statistics inherently difficult. However, it is because of this long-tail distribution that single molecule SERS can be performed. This will be discussed in a later chapter. 


\section{Chapter 4}

\section{Vibrational Pumping}

\subsection{Introduction}

Vibrational pumping is the creation of a vibrational population through Stokes Raman processes. Kneipp et al. [4] first proposed vibrational pumping as a means to measure SERS cross-sections by observing the power dependence of the anti-Stokes to Stokes intensity ratio. However, this technique failed to rule out other effects that may create a similar power dependency; namely laser heating and resonance effects [88-91]. Measurements by Kneipp et al. were taken at room temperature where the population created by vibrational pumping is only a small proportion compared with a large thermally excited population. Instead of measuring the power dependency of the intensity ratio, Maher et al. $[5,7,8]$ proposed that measuring the variation with temperature would give much more convincing results. At sufficiently low temperatures, the population created through thermal excitation is extremely small and the effects of vibrational pumping can be easily observed as an increase in the anti-Stokes intensity. As the temperature is increased, the mechanism of population excitation changes from being dominated by pumping to thermal excitation. By measuring how the anti-Stokes to Stokes intensity ratio changes as the temperature increases, it is possible to estimate the pumping cross-section (within certain approximations to be discussed later).

This chapter will give a detailed overview of the theory of vibrational pumping and also the experimental equipment and sample preparation techniques that will be used throughout this thesis. Also included is an investigation into 
the coupling between the vibrational modes of a molecule and how it is possible to use that knowledge to measure the vibrational lifetimes necessary for accurate cross-section estimates. Finally, an application of vibrational pumping will be discussed in the form of two-laser pumping which allows the measurement of the dispersion in the plasmon resonance distribution without calibrating the response of the system.

\subsection{The Theory of Vibrational Pumping}

\subsubsection{The anti-Stokes and Stokes Intensity}

As discussed in section 2.3.3, the Stokes and anti-Stokes intensities, for a single molecule, are dependent on the average vibrational population of the mode for each molecule, $n$, the Stokes or anti-Stokes differential cross-section $d \sigma / d \Omega$, and the laser power density $P_{L}$ :

$$
\begin{gathered}
I_{S}=\frac{d \sigma_{S}^{R}}{d \Omega} P_{L}, \\
I_{a S}=n \frac{d \sigma_{a S}^{R}}{d \Omega} P_{L} .
\end{gathered}
$$

The superscript $R$ on the cross-sections corresponds to radiative Stokes or anti-Stokes scattering in which the scattered light is detected in the far field. It is also possible to have non-radiative Raman scattering where the scattered light is absorbed by the metallic nano-particles. This will be discussed later in the chapter.

Due to the dependence on the vibrational population in equation 4.2 , the anti-Stokes intensity will typically be much smaller than the Stokes intensity. There are three processes that will affect the size of the population; thermal excitation, vibrational pumping (the population created through Stokes scattering), and relaxation. The thermal excitation rate will follow a Boltzmann distribution in relation to temperature, while the excitation rate, due to Stokes scattering, will depend on the Stokes cross-section and the incident photon density. The overall rate of change of the average vibrational population is therefore given by:

$$
\frac{d n}{d t}=\frac{\sigma_{S}^{T} P_{L}}{\hbar \omega_{L}}+\frac{\exp \left(-\hbar \omega_{\nu} / k_{B} T\right)}{\tau}-\frac{n}{\tau},
$$


where $\tau$ is the lifetime of the vibration, $\omega_{L}$ is the excitation frequency, and $\omega_{\nu}$ is the frequency of the vibration. Because the population will increase for both radiative and non-radiative Stokes scattering, it is the total Stokes cross-section, $\sigma_{S}^{T}$, that determines the pumped population. The total Stokes cross-section is the sum of the non-radiative Stokes cross-section and the radiative Stokes crosssection:

$$
\sigma_{S}^{T}=\sigma_{S}^{N R}+\sigma_{S}^{R} .
$$

In this case the radiative cross-section is integrated over all directions to ensure that the total cross-section is independent of the scattering direction.

There may also be a slight contribution to the pumping cross-section from fluorescence pumping [76,77], but this will only occur for resonant molecules. Fluorescence pumping refers to the vibrational population that is excited whenever the molecule decays from the first electronic state. The main difference between Stokes pumping and fluorescence pumping is that fluorescence pumping excites a population in all of the vibrational modes, while Stokes pumping only excites a population in Raman active modes. It is therefore unclear how much of a contribution fluorescence pumping has on the population for a single mode. Consequently, any vibrational pumping performed on resonant molecules may have a contribution due to fluorescence pumping. However, the fact that vibrational pumping has been observed for non-resonant molecules [1] means that Stokes scattering is a viable mechanism for creating a vibrational population.

The two excitation rates in equation 4.3 are independent of each other and, as a result, there are several properties that will determine the dominant term:

- The total Stokes cross-section. A large Stokes cross-section will result in a larger population created through Stokes processes. Experimentally, using a substrate with a large electromagnetic enhancement, or using molecules that are in resonance with the laser excitation, will greatly increase the Stokes cross-section (as discussed in the previous chapter). Under pumping conditions, this cross-section is different to the typical SERS Stokes cross-section, due to non-radiative processes. This will be addressed later in the chapter.

- The laser power density. Using a large power will increase the number of Stokes processes. However, photo-bleaching may become a problem. An investigation of the effects of photo-bleaching on vibrational pumping will 
be given later in this chapter.

- Temperature. The second term on the right in equation 4.3 ensures that the vibrational population, created via thermal excitation, is always equal to the Boltzmann factor. As a result, increasing the temperature will also increase the thermal population.

- The energy of the vibrational mode. For high energy modes, the thermal energy required to create a single vibration increases. As a result, the thermal population will be smaller for high energy modes.

- The vibrational lifetime. Unlike thermal excitation and spontaneous decay, the excitation rate via Stokes scattering is independent of the lifetime of the vibration. Consequently, a large population can be created via Stokes processes if the vibration remains for a long time before decaying.

Because the decay rate is proportional to the population, after sufficient time a molecule will eventually reach a steady state in which the average population is constant. Equation 4.3 will therefore tend to zero and we can write the vibrational population as:

$$
n=\frac{\tau \sigma_{S}^{T} P_{L}}{\hbar \omega_{L}}+\exp \left(-\frac{\hbar \omega_{\nu}}{k_{B} T}\right),
$$

where the first term is the vibrational pumping term and the second term is thermal excitation term. Combining this with equation 4.2, the single molecule anti-Stokes intensity can be written as:

$$
I_{a S}=\left[\frac{\tau \sigma_{S}^{T} P_{L}}{\hbar \omega_{L}}+\exp \left(-\frac{\hbar \omega_{\nu}}{k_{B} T}\right)\right] \frac{d \sigma_{a S}^{R}}{d \Omega} P_{L} .
$$

Since the focus of this chapter is vibrational pumping of large numbers of molecules (single molecule vibrational pumping will be re-addressed in chapter 6 ), the cross-sections can vary significantly between molecules due to the different plasmon resonances they experience. The overall Stokes intensity will therefore be the sum over all molecules:

$$
I_{S}=\sum_{i=1}^{N}\left(\frac{d \sigma_{S}^{R}}{d \Omega}\right)^{i} P_{L}=N\left\langle\frac{d \sigma_{S}^{R}}{d \Omega}\right\rangle P_{L},
$$

where $N$ is the total number of molecules contributing to the signal, and the 
triangular brackets represent an average over all molecules. Similarly, the antiStokes intensity can be written as:

$$
\begin{aligned}
I_{a S} & =\sum_{i=1}^{N} n^{i}\left(\frac{\sigma_{a S}^{R}}{d \Omega}\right)^{i} P_{L} \\
& =N\left[\frac{\tau\left\langle\sigma_{S}^{T} d \sigma_{a S}^{R} / d \Omega\right\rangle P_{L}}{\hbar \omega_{L}}+\left\langle d \sigma_{a S}^{R} / d \Omega\right\rangle \exp \left(-\frac{\hbar \omega_{\nu}}{k_{B} T}\right)\right] P_{L}
\end{aligned}
$$

In SERS, it is very difficult to estimate the number of molecules that are contributing to the signal as those closest to plasmon resonance hot-spots (discussed in the previous chapter) will dominate the SERS signal. However, because both the Stokes and anti-Stokes intensities are linearly dependent on the number of molecules, the ratio between them must be independent of $N$ :

$$
\rho=\frac{I_{a S}}{I_{S}}=\frac{\left\langle\sigma_{S}^{T} d \sigma_{a S}^{R} / d \Omega\right\rangle}{\left\langle d \sigma_{S}^{R} / d \Omega\right\rangle} \frac{\tau P_{L}}{\hbar \omega_{L}}+\frac{\left\langle d \sigma_{a S}^{R} / d \Omega\right\rangle}{\left\langle d \sigma_{S}^{R} / d \Omega\right\rangle} \exp \left(-\frac{\hbar \omega_{\nu}}{k_{B} T}\right) .
$$

This can be further simplified by introducing the ensemble asymmetry factor, $A^{E}$ :

$$
A^{E}=\frac{\left\langle d \sigma_{a S}^{R} / d \Omega\right\rangle}{\left\langle d \sigma_{S}^{R} / d \Omega\right\rangle},
$$

which is the ratio of the average anti-Stokes cross-section to the average Stokes cross-section. The asymmetry factor will be discussed in detail in section 4.2.4. In addition to the asymmetry factor, I will define the pumping cross-section as:

$$
\sigma_{\text {pump }}=\frac{\left\langle\sigma_{S}^{T} d \sigma_{a S}^{R} / d \Omega\right\rangle}{\left\langle d \sigma_{a S}^{R} / d \Omega\right\rangle} .
$$

The physical interpretation of this definition will be addressed in section 4.2.5. Equation 4.9 can now be written as:

$$
\rho=\frac{I_{a S}}{I_{S}}=A^{E}\left[\frac{\sigma_{\mathrm{pump}} \tau P_{L}}{\hbar \omega_{L}}+\exp \left(-\frac{\hbar \omega_{\nu}}{k_{B} T}\right)\right] .
$$

\subsubsection{Laser Heating}

There are two variables in equation 4.12 that we have a large amount of control over; the incident power and the temperature. Adjusting the incident power 
will allow us to control the pumping rate, while adjusting the temperature will allow us to control the rate of thermal excitation. By varying either of these parameters, we should be able to control which of the two excitation processes is the dominant contribution to the vibrational population. However, the effective temperature of a molecule under SERS conditions may not be the same as the temperature of its environment due to heating by the laser.

The first reported observations of vibrational pumping in SERS looked at how the anti-Stokes to Stokes ratio varied with $P_{L}$ at room temperature [4]. If the ratio is plotted as a function of laser power, equation 4.12 states that we will observe a linear dependence which will have a slope that is dependent on $\tau$ and $\sigma_{\text {pump. }}$. A value for the pumping cross-section can therefore be estimated if the vibrational lifetime is known. However, at room temperature $\left(\sim 20^{\circ} \mathrm{C}\right)$, the population created through thermal excitation will be substantially larger than the pumping population (approximately a factor of 200 larger for an incident power of $1 \mathrm{~mW}$ over a $1 \mu \mathrm{m}^{2}$ spot). As a result, it is very difficult to measure the small change in population created via vibrational pumping. Nevertheless, reasonably accurate values for the pumping cross-section $\left(\sim 10^{-16} \mathrm{~cm}^{2}\right)$ were obtained (assuming a $\tau$ of $10 \mathrm{ps}$ ), but due to poor normalisation techniques, extremely large enhancement factors were predicted $\left(\sim 10^{14}-10^{15}\right)$.

In addition to the large enhancement factor estimates, the initial pumping observations failed to rule out the contribution of laser heating. The effective temperature experienced by the molecules in equation 4.12 can be written as:

$$
T=T_{0}+\Delta T
$$

where $T_{0}$ is the substrate temperature and $\Delta T$ is the increase in temperature due to laser heating. Because heat diffusion and transfer models are linear, it is reasonable to assume that the increase in temperature due to laser heating is linearly proportional to the laser intensity:

$$
\Delta T \approx a P_{L}
$$

where $a$ is the proportionality constant. Equation 4.14 can be substituted into equation 4.13 to give:

$$
T=T_{0}+a P_{L} .
$$

If we assume that $\Delta T$ is much smaller than $T_{0}$, we can write the thermal exci- 
tation term in equation 4.12 as:

$$
\begin{aligned}
\rho_{\text {thermal }} & =A^{E} \exp \left(-\frac{\hbar \omega_{\nu}}{k_{B}\left(T_{0}+\Delta T\right)}\right) \\
& \approx A^{E} \exp \left(-\frac{\hbar \omega_{\nu}}{k_{B} T_{0}}+\frac{\hbar \omega_{\nu} \Delta T}{k_{B} T_{0}^{2}}\right) \\
& =A^{E} \exp \left(-\frac{\hbar \omega_{\nu}}{k_{B} T_{0}}\right) \exp \left(\frac{\hbar \omega_{\nu} \Delta T}{k_{B} T_{0}^{2}}\right) \\
& \approx A^{E} \exp \left(-\frac{\hbar \omega_{\nu}}{k_{B} T_{0}}\right)\left(1+\frac{\hbar \omega_{\nu} a P_{L}}{k_{B} T_{0}^{2}}\right),
\end{aligned}
$$

which is true as long as $\Delta T / T_{0}<<k_{B} T_{0} / \hbar \omega$. As a result, the thermally excited population may also be linearly dependent on the laser intensity. It is therefore very difficult to conclude whether a linear dependence between $\rho$ and $P_{L}$ is evidence for pumping or laser heating. In hindsight, the similarity between the pumping cross-sections estimated by Kneipp et al. and Maher et al. suggests that vibrational pumping was in fact observed.

It is important to note that the effects of laser heating will be present at all temperatures [88]. In fact, the lower the temperature the greater the effect that laser heating has on the thermally excited population. However, at sufficiently low temperatures, the pumped population will always be much larger than the thermally excited population, even with the contribution from laser heating.

\subsubsection{The Temperature Dependence}

Due to problems with laser heating and the low vibrational population at room temperature, recent measurements of vibrational pumping have investigated how $\rho$ varies with temperature $[7,8]$. At sufficiently low temperatures, the pumping term in equation 4.12 will be the dominant contribution to the population. This has the consequence that the relative intensity of the anti-Stokes peaks will be dependent on their cross-sections instead of following an exponential decrease with increasing energy as we see at room temperature (due to the Boltzmann distribution in the thermally excited population). Maher et al. $[7,8,92]$ have shown that this is indeed the case at temperatures as low as $10 \mathrm{~K}$ for the common SERS molecules Rhodamine 6G, Crystal Violet, Nile Blue and Benzene Thiol.

Figure 4.1 shows how the anti-Stokes to Stokes intensity ratio for a single mode changes with increasing temperature. At low temperatures, the mode 


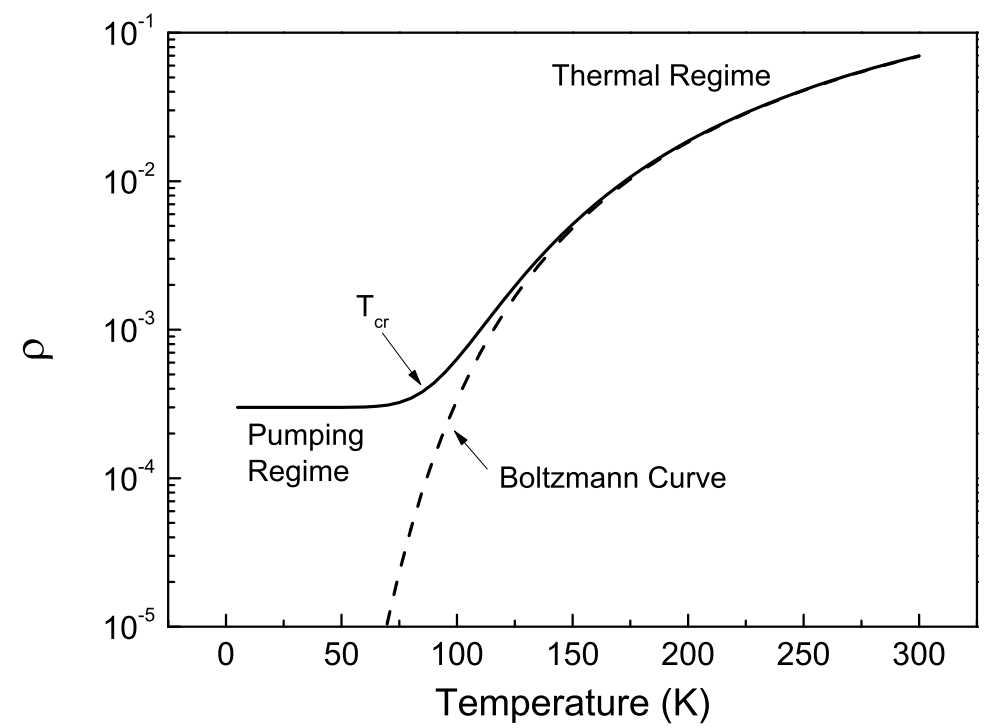

Figure 4.1: A theoretical plot of the temperature dependence of the anti-Stokes to Stokes intensity ratio, $\rho$, for a single Raman mode. $\rho$ is plotted on a log scale due to the large variation in the thermally excited regime.

is in what we call the "pumping regime" where the vibrational population is created mainly through vibrational pumping. This has the result that both the anti-Stokes and Stokes signals do not change in intensity as the temperature varies. However, as the temperature is increased there is a point in which the thermally excited population becomes comparable to the pumped population. This is called the critical (or cross-over) temperature and given by:

$$
T_{\mathrm{cr}}=\frac{\hbar \omega_{\nu}}{k_{B}}\left[\ln \left(\frac{\hbar \omega_{L}}{\tau \sigma_{\mathrm{pump}} P_{L}}\right)\right]^{-1},
$$

which is derived by setting the pumping population equal to the thermal population. The critical temperature is very dependent on the experimental set-up as it varies with several parameters; the mode energy, the incident photon density, the pumping cross-section and the lifetime of the mode. Above the critical temperature, the mode will be in the thermal regime in which thermal excitation will be the dominant mechanism. Due to the exponential dependence of the thermal population, the transition from a regime completely dominated by pumping to one dominated by thermal excitation will occur over a small temperature range. The pumping regime will however be larger for higher energy 


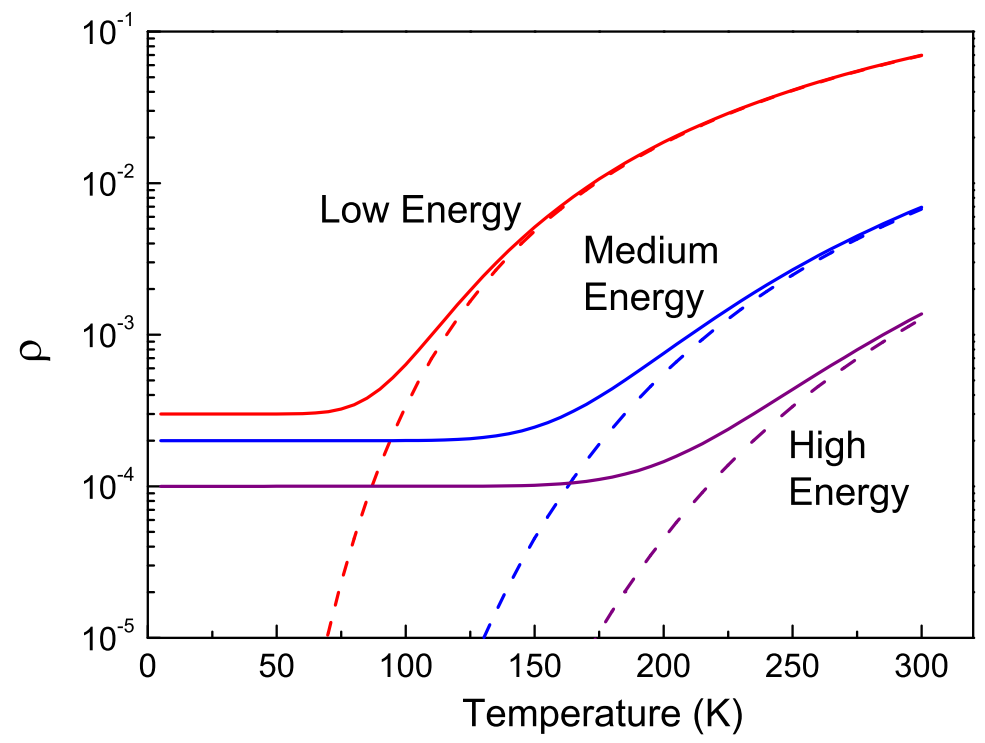

Figure 4.2: $\quad$ Plots of the temperature dependence of $\rho$ for a low, medium and high energy mode.

modes (see figure 4.2).

Due to the Boltzmann term in the thermal population, $\rho$ will often vary over several orders of magnitude. As a result, it is often the case that $\ln (\rho)$ is plotted as a function of $T$ and fitted with the function:

$$
\ln (\rho)=\ln \left(A^{E}\right)+\ln \left[b+\exp \left(-\frac{\hbar \omega_{\nu}}{k_{B} T}\right)\right]
$$

where:

$$
b=\frac{\tau \sigma_{\mathrm{pump}} P_{L}}{\hbar \omega_{L}}
$$

This also has the advantage that we can estimate both the pumping cross-section and asymmetry factor from the fitting parameters. Using this technique, Maher et al. have managed to measure pumping cross-sections for many common SERS probes $[7,92]$.

\subsubsection{The Asymmetry Factor and Resonance Effects}

Let us now return to the physical interpretation of the asymmetry factor [93]. In the previous section I gave a technique for estimating the asymmetry factor 


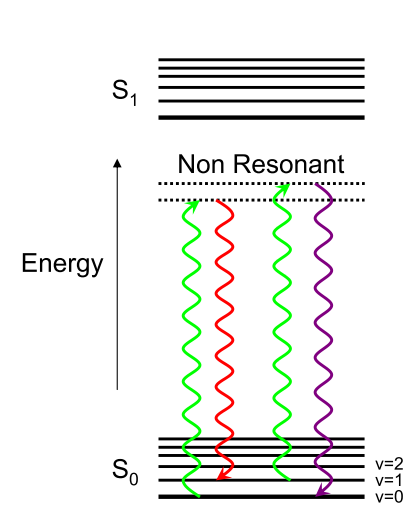

(a) $\mathrm{A} \sim 1$

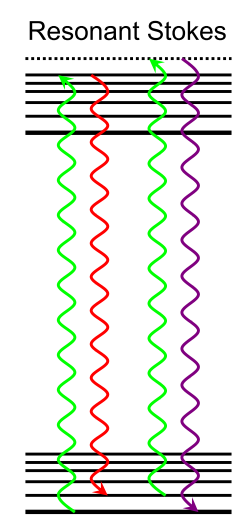

(b) $A<1$

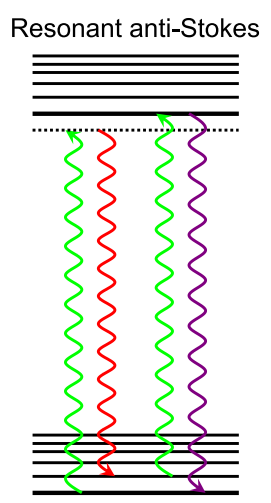

(c) $A>1$

Figure 4.3: A Jablonski diagram of Raman scattering when (a) neither scattering mechanisms is favoured, (b) Stokes scattering is favoured $(A<1)$, and (c) anti-Stokes scattering is favoured $(A>1)$.

from the temperature dependence of $\rho$. However, once we have obtained a value, what information can we conclude from it? The asymmetry factor is defined as the ratio of the average anti-Stokes cross-section to the average Stokes cross-section. If we take the simplest case of a single isolated molecule under non-resonant conditions, the Stokes and anti-Stokes cross-section will be similar (only different due to the $\omega^{4}$ dependence) resulting in an asymmetry of $A \sim 1$ (see figure 4.3(a)). For the case of a molecule that is close to resonance, either Stokes or anti-Stokes scattering will be favoured resulting in an asymmetry that is not unity. If Stokes scattering is favoured (figure 4.3(b)) the asymmetry will be less than one and if anti-Stokes scattering is favoured (figure 4.3(c)) the asymmetry will be larger than one.

For a large number of molecules, under non-SERS conditions, the asymmetry factor will be exactly the same as for a single isolated molecule as only the incident wavelength and Raman shift will affect the asymmetry. However, once the molecules are placed close to a metallic substrate there will be an additional contribution to the asymmetry factor due to the plasmon resonance. In section 3.2.1 I explained that the SERS enhancement factor depends not only on the incident wavelength but also on the scattered wavelength. Furthermore, in the previous chapter we saw that there is a wavelength dependence on the strength of the plasmon resonance which can vary greatly with the local environment of the observed molecule. As a result, the anti-Stokes and Stokes cross-sections will be different as they have different scattering wavelengths. Under SERS conditions, 
there will therefore be two contributions to the asymmetry factor; the absorption resonance which will affect each molecule equally, and the plasmon resonance which will be different for each molecule. Consequently, the total asymmetry factor will be the absorption resonance asymmetry plus the average asymmetry created from the plasmon resonances.

\subsubsection{The Pumping Cross-Section and Spatial Averaging}

In section 4.2.1, I defined the pumping cross-section as:

$$
\sigma_{\text {pump }}=\frac{\left\langle\sigma_{S}^{T} d \sigma_{a S}^{R} / d \Omega\right\rangle}{\left\langle d \sigma_{a S}^{R} / d \Omega\right\rangle} .
$$

For a single molecule, this can be simplified to the total Stokes cross-section but, due to the spatial averaging, this is not the case for many molecules. Instead we must consider the enhancement factors that affect each of these cross-sections. Both radiative and non-radiative Stokes scattering receive a local field enhancement $M_{\mathrm{Loc}}\left(\omega_{L}\right)$, at the laser frequency, in the excitation step of the Raman process. However, while the radiative Stokes process is enhanced by $M_{\operatorname{Rad}}\left(\omega_{S}\right)$ in the emission step, the non-radiative Stokes process is enhanced by $M_{\mathrm{NR}}$ (discussed in section 3.2.2). The total Stokes cross-section will therefore be given by:

$$
\begin{aligned}
\sigma_{S}^{T}\left(\omega_{S}\right) & =\sigma_{S}^{R}\left(\omega_{S}\right)+\sigma_{S}^{N R}\left(\omega_{S}\right) \\
& =M_{\mathrm{Loc}}\left(\omega_{L}\right) M_{\mathrm{Rad}}\left(\omega_{S}\right) \sigma_{\mathrm{Raman}}+M_{\mathrm{Loc}}\left(\omega_{L}\right) M_{\mathrm{NR}}\left(\omega_{S}\right) \sigma_{\mathrm{Raman}} \\
& =M_{\mathrm{Loc}}\left(\omega_{L}\right) M_{\mathrm{Tot}}\left(\omega_{S}\right) \sigma_{\mathrm{Raman}}
\end{aligned}
$$

where $M_{\text {Tot }}\left(\omega_{S}\right)=M_{\mathrm{Rad}}\left(\omega_{S}\right)+M_{\mathrm{NR}}\left(\omega_{S}\right)$. In this case $M_{\mathrm{Rad}}\left(\omega_{S}\right)$ has been averaged over all scattering directions and $\sigma_{\text {Raman }}$ is the Raman cross-section integrated over all directions.

If we assume that $M_{\mathrm{Rad}} \approx M_{\mathrm{Loc}}$ (see section 3.2.1) and ignore the wavelength dependence of the enhancement factors, then:

$$
\sigma_{\text {pump }} \approx \frac{\left\langle M_{\text {Loc }}^{3} M_{\text {Tot }}\right\rangle}{\left\langle M_{\text {Loc }}^{2}\right\rangle} \sigma_{\text {Raman }} .
$$

If radiative Stokes scattering was more likely than non-radiative Stokes scattering, it can be shown that the pumping cross-section would be a slight under- 
estimation of the radiative Stokes cross-section for molecules located in hotspots [7]. However, for molecules directly adsorbed onto the surface of metals, non-radiative Stokes scattering typically dominates (this will be shown in chapter 6). In this scenario, the interpretation of the pumping cross-section is not so straight forward. If all of the molecules are bound identically to the surface of the metal, the non-radiative enhancement factor should be approximately constant. The pumping cross-section will therefore be given by:

$$
\sigma_{\text {pump }} \approx M_{N R} \frac{\left\langle M_{\text {Loc }}^{3}\right\rangle}{\left\langle M_{\text {Loc }}^{2}\right\rangle} \sigma_{\text {Raman }}
$$

In the last chapter, it was shown that the local field enhancement factor close to a plasmon resonance could vary by several orders of magnitude if the position was shifted by a couple of nanometers, resulting in a maximum enhancement factor that could be $10^{2}-10^{3}$ times larger than regions further away from the plasmon resonance. The molecules located in the hot-spots will therefore have much larger pumping cross-sections than the other molecules.

Let us consider a simple example of 1000 molecules, where one molecule is located in a hot-spot with a local field enhancement factor that is 100 times larger than the rest. The pumping cross-section will therefore be:

$$
\begin{aligned}
\sigma_{\text {pump }} & =M_{\mathrm{NR}} \frac{\left(\left(100 M_{\mathrm{Loc}}\right)^{3}+999 M_{\mathrm{Loc}}^{3}\right) / 1000}{\left(\left(100 M_{\mathrm{Loc}}\right)^{2}+999 M_{\mathrm{Loc}}^{2}\right) / 1000} \sigma_{\mathrm{Raman}} \\
& =M_{\mathrm{NR}} \frac{1 \times 10^{6} M_{\mathrm{Loc}}^{3}}{1.1 \times 10^{4} M_{\mathrm{Loc}}^{2}} \sigma_{\mathrm{Raman}} \\
& =91 M_{\mathrm{NR}} M_{\mathrm{Loc}} \sigma_{\mathrm{Raman}} \\
& \approx\left(\sigma_{S}^{T}\right)^{H S}
\end{aligned}
$$

where $\left(\sigma_{S}^{T}\right)^{H S}$ is the total Stokes cross-section of the molecule located in the hotspot. The pumping cross-section will therefore be a better approximation of the total Stokes cross-section if $\sigma^{H S} / \sigma$ is much larger than the number of molecules not in hot-spots. However, due to the long-tail distribution of enhancement factors, it is very difficult to properly interpret the pumping cross-section and it may only be achievable under single molecule conditions where the spatial averaging does not appear. 


\subsubsection{The Population Lifetime}

By fitting the function in equation 4.17 to the temperature dependence of the anti-Stokes to Stokes intensity ratio, we can calculate a value for $b=$ $\tau \sigma_{\text {pump }} P_{L} / \hbar \omega_{L}$. However, before we can convert this into the pumping crosssection, we need a reliable technique for estimating the vibrational lifetime $\tau$. In some cases, time-resolved measurements [94-97] have been made to directly measure the vibrational lifetime of some common SERS probes [98].

In reference $[7,92]$ we proposed that the lifetime could be estimated from the width of the Raman peak. The Heisenberg uncertainty principle states that the uncertainty in the mode energy is related to the lifetime by:

$$
\tau \Gamma \sim \hbar
$$

where $\Gamma$ is the lifetime contribution to the full-width half-maximum of the Stokes peak. There are however additional peak width contributions:

- Dephasing Lifetime: There is an additional lifetime associated with a vibrational mode called the dephasing lifetime [99,100]. Often the relaxation lifetime is referred to as $T_{1}$ and the dephasing lifetime is $T_{2}$. The dephasing lifetime is defined as the length of time required for the vibration to lose coherence.

- Overlapping Raman modes: If there are several Raman modes close together, a single peak may actually be several modes that are sufficiently close that their natural line widths overlap.

- Inhomogeous Broadening: This is similar to overlapping Raman modes but instead of having several modes close together, a single mode is slightly shifted in energy for different molecules. This results in a Raman peak that has a width that is larger than the line width expected for a single molecule. The size of the shift will depend on the interaction of the molecule with its local environment and, hence, different molecules will experience different shifts in the Raman mode energy. This effect has been studied in detail in reference [101] using single molecule SERS.

Inhomogeneous broadening and overlapping Raman modes will only account for a discrepancy of 2 or 3 in the lifetime, but the dephasing lifetime may cause a significant broadening of the peak if it is shorter than the relaxation lifetime. As a result, it is important to distinguish which modes have a shorter 
relaxation lifetime than dephasing lifetime. The peak width of these modes will therefore be a good estimate of $\tau$ within a factor of 2 or 3 (due to inhomogeneous broadening and overlapping Raman modes). A technique for identifying exactly which lifetime process is the dominant mechanism will be investigated later in this chapter (section 4.4).

Typically, we will only be concerned with order of magnitude estimates of the pumping cross-section and the population lifetime will be assumed to be $\sim 1 \mathrm{ps}$ [92]. This is in agreement with the characteristic peak widths for the SERS analytes observed in this thesis.

\subsubsection{Photo-bleaching}

Under pumping conditions (when the population is dominated by vibrational pumping), the molecules located close to hot-spots will dominate the anti-Stokes intensity due to the cubic dependence on the local field enhancement factor. The Stokes intensity, on the other hand, will have a contribution from molecules that do not receive a particularly large enhancement as the Stokes intensity only has a square dependence on the local field enhancement. Because photo-bleaching is proportional to the overall enhancement (due to increased absorption and other effects that are not well understood), molecules with the largest cross-sections will photo-bleach fastest. Consequently, as the hot-spot molecules are bleached, the anti-Stokes intensity will decrease faster than the Stokes intensity. This results in an anti-Stokes to Stokes intensity ratio that decreases with time. The decrease in $\rho$ can be interpreted as a decrease in the pumping cross-section with time, which is not unexpected as the molecules with the largest enhancement factors have the greatest chance of photo-bleaching.

At high temperatures, when thermally excited vibrations dominate the population, both the anti-Stokes and Stokes intensities are linearly related to the cross-section. The intensity ratio will therefore be independent of photo-bleaching and not vary with time. An in-depth discussion on the effects of photo-bleaching on vibrational pumping can be found in reference [102].

\subsection{Experimental Set-Up}

This section is dedicated to an in-depth overview of the experimental set-up used throughout the thesis. This includes the Raman spectrometers, the cryostats and the sample preparation method. Instead of repeating the information below, 


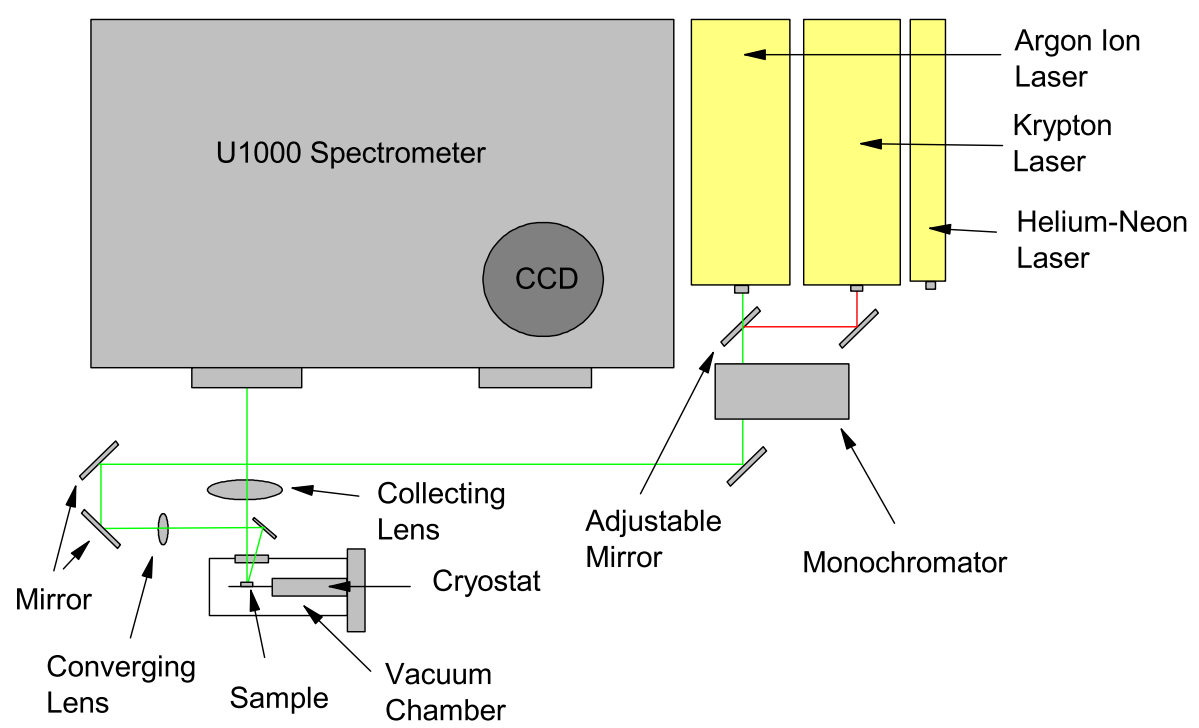

Figure 4.4: A schematic layout of the U1000 system. The system is set up for the argon and krypton lasers using the adjustable mirror to select which laser is used. The internal optics of the spectrometer are shown in figure 4.5.

this section will be referenced whenever an explanation of a new experiment is given.

\subsubsection{Raman Spectrometers}

Two Raman spectrometers were used over the course of my $\mathrm{PhD}$ research, a double additive U1000 Jobin-Yvon spectrometer and a LabRam Raman system. The choice of system was dependent on the types of measurements that were required.

\section{The Double Additive U1000 Spectrometer}

There are three laser set up for use with the U1000 spectrometer, an argon-ion laser with several lines in the green and blue, a krypton laser with two red lines, $647 \mathrm{~nm}$ and $676 \mathrm{~nm}$, and a helium-neon laser with a line at $633 \mathrm{~nm}$. The layout of this system is shown in figure 4.4. The laser is first passed through a monochromator to remove the plasma lines and then focused on a solid sample using a converging lens. The scattered light is collected using a second lens and focused on the spectrometer. Once the light passes into the spectrometer, it goes through a series of optical stages (see figure 4.5) in which the blazed holographic 


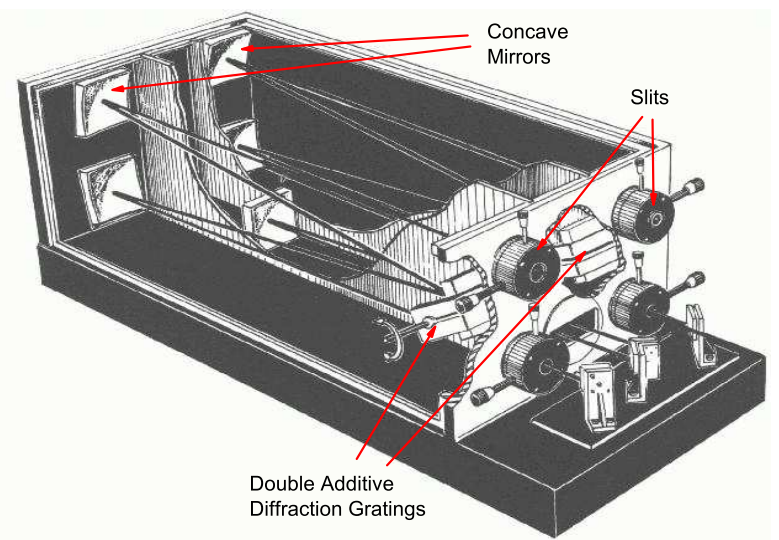

Figure 4.5: A schematic of the internal optics of a U1000 spectrometer. Included are two diffraction gratings that are double additive. There are also five concave mirrors that either parallelize the light for the diffraction gratings or focus the light so that it can pass through the slits. The actual optics of the spectrometer used in this thesis are slightly different as the light from the second diffraction grating is focused on a $C C D$ that is above the system, but for our purposes this diagram is sufficient for an understanding of the internal workings of the U1000.

plane gratings separate the light in a double additive fashion. Finally it is focused on a liquid nitrogen cooled CCD which is read by the computer and displayed using the program LabSpec [103].

The main advantage of the U1000 spectrometer is that it allows high resolution measurements of Raman peaks. Because the spectrometer is double additive, it is possible to focus on an extremely small region of the signal. In fact a single peak can be observed, allowing the accurate measurement of properties such as peak widths and locations. However, it is not possible to simultaneously measure numerous modes without modifying the internal operation of the spectrometer. This a key disadvantage in vibrational pumping where accurate measurements of both the anti-Stokes and Stokes peaks are needed, and having to constantly shift the position of the gratings may lead to discrepancies.

\section{The LabRam System}

As with the U1000 system, the Jobin-Yvon LabRam is connected to an argon-ion and a helium-neon laser. Additionally, the LabRam system has a UV heliumcadmium laser with a line at $325 \mathrm{~nm}$ and an IR laser diode at $785 \mathrm{~nm}$. However, all of the measurements performed in this thesis were on dye molecules with resonances in the visible region of the electromagnetic spectrum so the IR and 


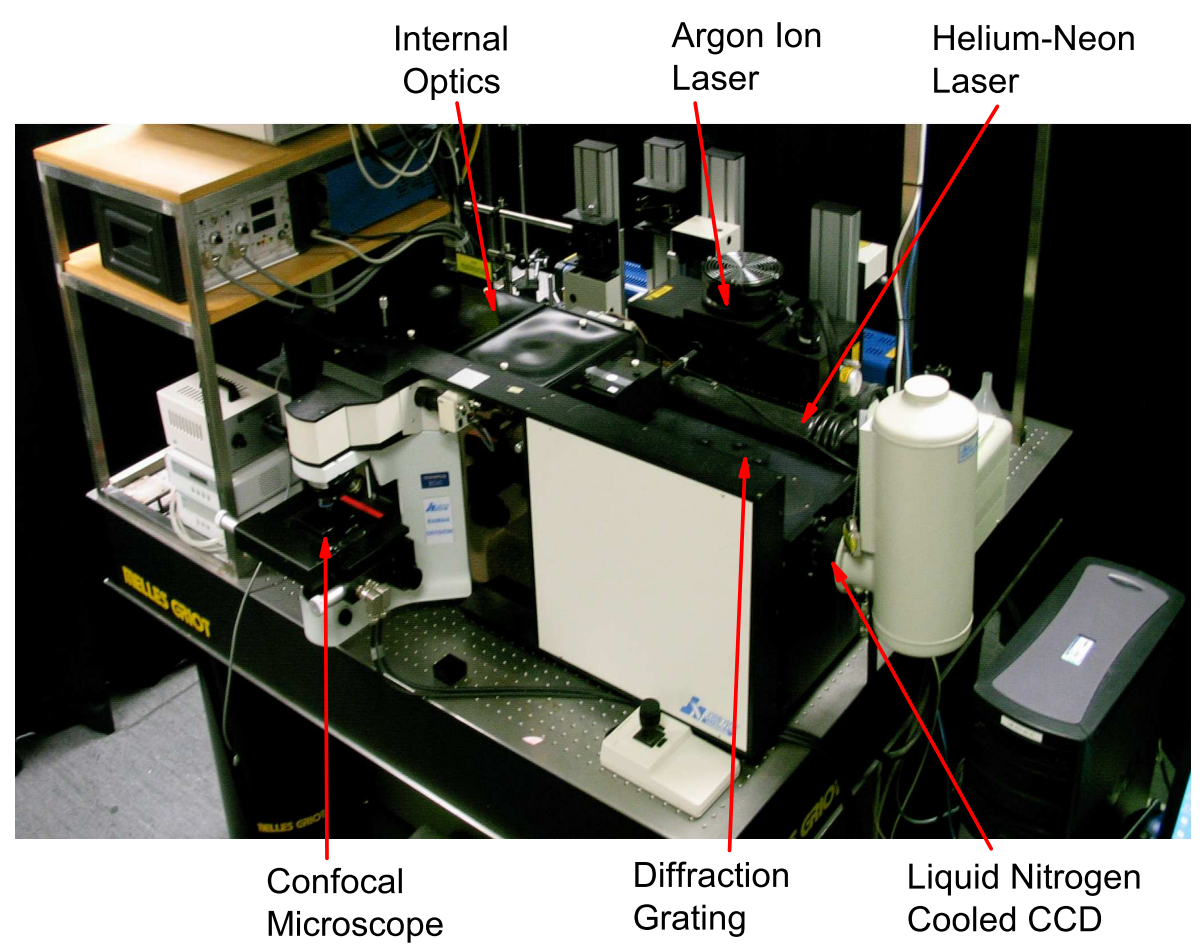

Figure 4.6: A photograph of the LabRam system. Shown are the lasers used in this thesis (the argon ion and helium-neon lasers), the confocal microscope where the incident light is focused and the scattered light is collected, and the location of the CCD, diffraction grating and internal optics. The grating and optics are easily accessible by simply removing the cover above them. 


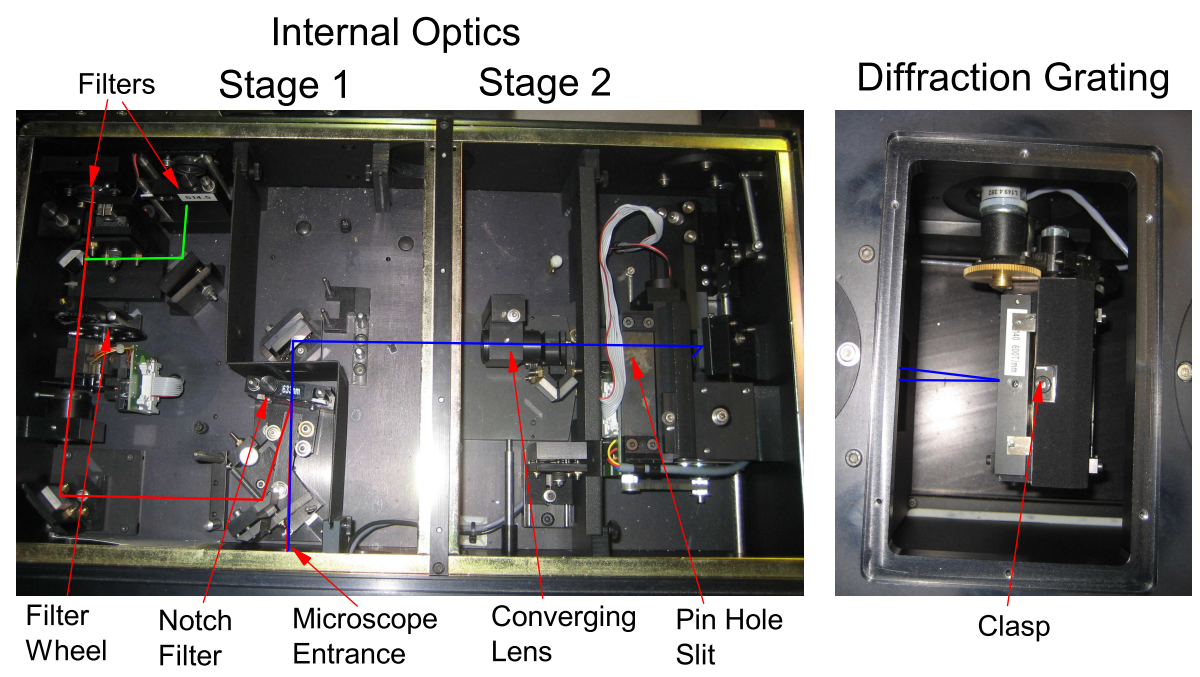

Figure 4.7: The internal optics of the LabRam system. The key features are labelled and explained in the text. In this case, the full path of the $633 \mathrm{~nm}$ (red line) laser line is shown. If we wish to use the $514 \mathrm{~nm}$ (green line) laser line, the mirror at the point of intersection needs to be lowered. The scattered light is shown in blue which passes through the notch filter and into the second stage. The diffraction grating can be easily replaced after the clasp is removed.

UV lasers were not used. The layout of the LabRam system is shown in figure 4.6. The laser is initially directed to the internal optics of the spectrometer (see figure 4.7) which filters the plasma lines and performs a power modification using the filter wheel. Using a notch filter (a filter which only reflects light at a specific wavelength) with the same wavelength as the laser, the laser is redirected into the Olympus BX41 confocal microscope. The light is then focused on the sample using interchangeable objectives. The scattered light is collected with the same objective and follows the same path as the incident light. However, the Raman-shifted scattered photons pass through the notch filter into the second stage of the internal optics. At this stage, a converging lens focuses the light through a pin hole slit which is then sent down to a parallelizing mirror and onto a single grating which disperses the light into its wavelengths. Finally the dispersed light is focused onto the liquid nitrogen cooled CCD which is read by the computer and displayed using LabSpec.

The main advantage of the LabRam compared to the U1000 is the amount of control the user has over the system. With the U1000, we are restricted to using solid samples and the size of the observed region is defined by the focusing 
lens. For the LabRam, however, there are many different objectives that can be used which will affect the size of the laser spot on the sample and the collecting efficiency of the scattered light. There are also immersion objectives that are designed for use with liquid samples. Furthermore, the LabRam is designed so that the user has easy access to the grating which can then be replaced with another. This allows us to take both high and low resolution measurements including simultaneous measurements of both the anti-Stokes and Strokes signals for many modes (depending on the incident wavelength). However, because the LabRam only uses one grating to disperse the light, it will never obtain the high resolution that can be achieved with the U1000.

Attached to the confocal microscope is a sample holding stage that can be adjusted in all three dimensions. Furthermore, the stage can be set-up so that spectra can be measured as the position changes allowing us to perform spatial mapping measurements of dry samples. This is very important for vibrational pumping as dry samples must be used due to the low temperature requirements.

Another important difference between the two systems is the use of the notch filter. Even if we could undertake low resolution measurements on the U1000, it is not possible to observe both the anti-Stokes and Stokes signal as this will directly expose the CCD to the laser which may cause damage. Using a notch filter avoids this problem, as the scattered light at the same wavelength as the laser is reflected while the Raman-shifted photons are able to pass through to the CCD, allowing the grating to be centred on the laser wavelength.

\subsubsection{Cryostats}

In order to get the temperature-dependent measurements required to observe vibrational pumping, a cryostat is needed. For the U1000 system, a closed cycle helium cryostat is set up which contains a copper mount that samples can be placed on using a highly thermal conductive silver paste. The sample is then isolated within a windowed steel chamber and pumped down to low pressures using a roughing and diffusion pump cycle. Once the chamber is evacuated, the temperature of the sample mount is then adjusted with the cryostat. Using this system it is possible to reach temperatures that range from $10 \mathrm{~K}$ to well above room temperature. The fact that the vacuum chamber is windowed means that it is possible to perform optical measurements by focusing the laser through the window.

For the LabRam, a LinKam THMS600 cryostat is used along with a LNP94 

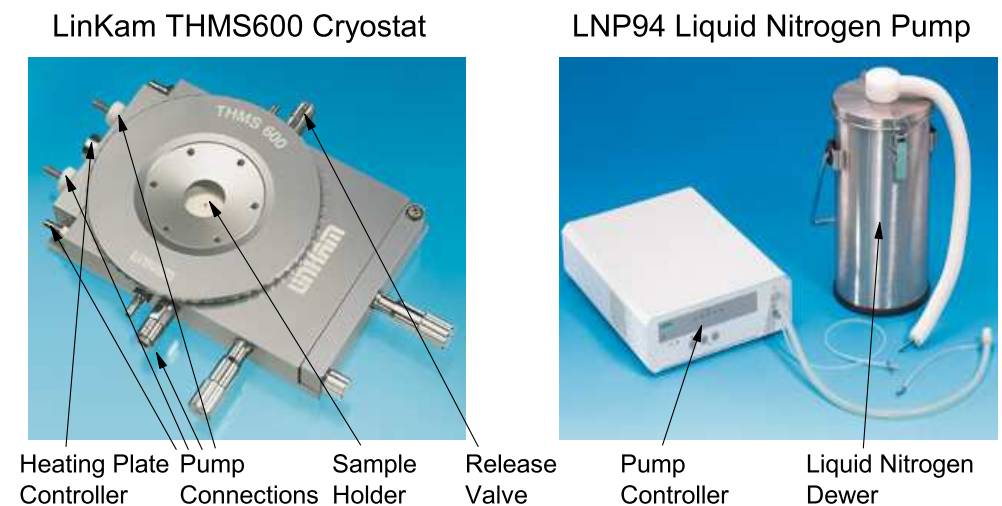

Figure 4.8: The cyrostat equipment for the LabRam system consisting of the LinKam THMS600 cryostat and LNP94 liquid nitrogen pump. The pump consists of a liquid nitrogen dewer and a controller for adjusting the pumping (cooling) rate. The controller is connected to the cryostat at the pump connections.

liquid nitrogen pump (see figure 4.8). The sample is mounted on a $0.17 \mathrm{~mm}$ cover slip which is on a highly polished silver heating element. Once the sample is mounted, the cryostat is sealed and the liquid nitrogen pump is used to create a purely nitrogen gas atmosphere while evacuating the initial atmosphere through the release valve. This allows us to decrease the temperature of the sample without ice crystals forming. The liquid nitrogen pump continuously pumps nitrogen gas into the chamber allowing the precise control of cooling rates as fast as $130^{\circ} \mathrm{C} / \mathrm{min}$ or as slow as $0.1^{\circ} \mathrm{C} / \mathrm{min}$. However, the lowest possible temperature reachable with this system is $77 \mathrm{~K}$ which may not be low enough to see pumping of the lowest energy vibrations. The sample can be heated up again using the silver heating element that is directly beneath the sample and is controlled by a second controlling unit (not shown in the figure). A weakness of using the cryostat on this system is that we need to use objectives with relatively low numerical apertures. Consequently, the efficiency of collecting the scattered light is relatively low. On the other hand, this is the only system that allows mappings, which are crucial for some of the results presented in this thesis.

\subsubsection{Sample Preparation}

While much of the sample preparation process is dependent on the experiment being performed, there are some steps that are consistent throughout the thesis and to save the need for repeating them, they will be explained here. Each 
sample consists of one or two SERS active dyes (most commonly Rhodamine 6G (RH6G), Nile Blue (NB) and Crystal Violet (CV)) which are diluted to the appropriate concentration using distilled water. Before the dye is added, a solution is prepared consisting of $500 \mathrm{~mL}$ of Lee and Meisel Ag colloids [104] and $500 \mathrm{~mL}$ of potassium chloride $(\mathrm{KCl})$ such that the final concentration is $2 \times 10^{11}$ colloids per $\mathrm{cm}^{3}$ and $10 \mathrm{mM}$ of $\mathrm{KCl}$. The purpose of the $\mathrm{KCl}$ is to shield the coulomb repulsion that the silver colloids (which are negatively charged) apply to each other. When the $\mathrm{KCl}$ is added, the potassium and chlorine ions separate and are free to move around. The charges will allow the colloids to get close enough to each other that van der Waals forces will cause them to "stick". The colloids are typically left for around 15 minutes to aggregate before the dye is added. However, there will be cases in which the dye is added to the colloids before the $\mathrm{KCl}$. Once the SERS dye is added, the molecules will bind to the surface of the colloids and in the gaps between them which is where the largest enhancements occur (as explained in the previous chapter).

At this stage the sample can be measured as a liquid sample using the LabRam system and an immersion objective. However, there cannot be any water molecules in the solution if low temperature pumping measurements are to be performed. A "dry" sample must therefore be used for these measurements. Taking a silicon wafer, we first coat it with Poly L-lysine ( $0.1 \%$ by weight) and leave it under a heating lamp for 5 minutes after which time the remaining liquid is syphoned off using a pipette. This leaves a thin layer of Poly L-lysine on the surface of the silicon which the silver colloids will attach to. The solution of SERS dye and aggregated colloids is then placed on the silicon and left under the heating lamp. The solution can be completely dried onto the silicon or it may be syphoned off after a certain amount of time. The final sample will consist of colloidal aggregates which are separated from each other by a distance that is dependent on the drying time of the solution. The effect of the drying time will become obvious in later chapters. Finally, the sample can be placed in the cryostat and pumping measurements can be performed.

Obviously vibrational pumping measurements will need to be performed with a dry sample, but there may be situations, at room temperature, where one sample type may be more suitable than the other. With a liquid sample, Brownian motion will cause the colloids to move in and out of the scattering volume of the laser and, hence, the molecules contributing to the SERS signal will change with time. As a result, many SERS events can be measured purely by taking time series measurements. For example, taking a $1 \mathrm{~s}$ SERS spectrum 
every $10 \mathrm{~s}$ for 1 hour. This does not occur for dry samples as the colloids are fixed on the surface of the silicon wafer. As a result, the location of the laser must be changed in the form of a spatial mapping to observe many different SERS events. However, if we wish to observe the evolution in the SERS signal for a specific region of the substrate, a dry sample must be used.

\subsection{Anharmonic Coupling of Vibrational States and Estimations of the Population Lifetime}

Obtaining an accurate value for the population lifetime is critical when estimating the pumping cross-section. Previously we noted that there are several contributions to the Raman line-width that create difficulties in estimating the population lifetime. This problem of estimating the contributions to the linewidth is a well established problem in spectroscopy [105]. The aim of this section is to investigate the anharmonic coupling between vibrational modes and hence obtain a more accurate estimate of the population lifetime and the pumping cross section. The dominant line-width contribution will be investigated for the main vibrational modes of NB and RH6G.

If we ignore the effects of inhomogeneous broadening and overlapping peaks (which will only cause a discrepancy of a factor of two or three in the lifetime), there are two contributions to the peak-width for a given mode: the relaxation lifetime $\left(T_{1}\right)$ which is the lifetime we need to estimate the pumping cross-section, and the dephasing lifetime $\left(T_{2}\right)$. Whichever lifetime is shortest will determine the peak-width.

\subsubsection{Anharmonic Coupling of Vibrational States}

Vibrational relaxation can only take place if energy is conserved. The lowest order anharmonic interactions for a vibration of frequency $\omega$ are shown in figure 4.9 in which the excited mode interacts with two other vibrational states within the same molecule. Figure 4.9(a) is the Feynman diagram for the decay of a vibration into two lower energy vibrations in which $\omega=\omega_{1}+\omega_{2}$ so that energy is conserved. This decay route results in the relaxation of the original state and hence is the main contribution (to first order) to $T_{1}$. This is an example of intramolecular vibrational relaxation (IVR). It is also possible for the vibration to decay via vibrations in the environment (also called the bath) 

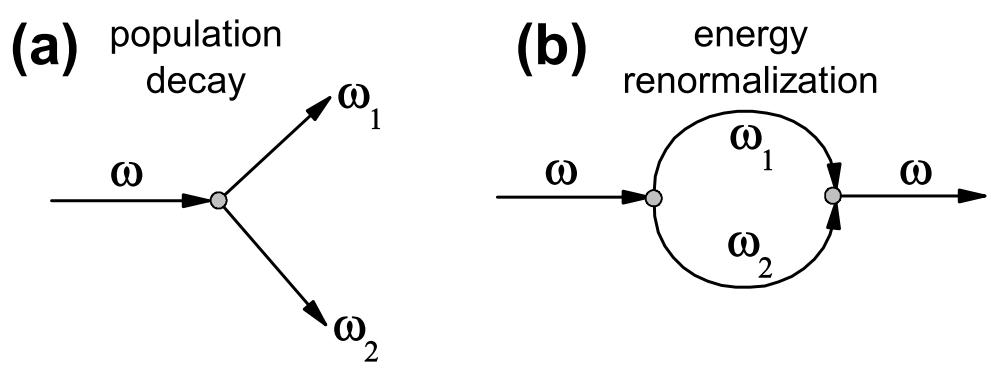

Figure 4.9: Feynmann diagrams for the lowest order anharmonic interactions for a vibration with frequency $\omega$. (a) shows the standard decay of a vibration $\omega$ into two vibrations $\omega_{1}$ and $\omega_{2}$ while (b) shows the next order process which is similar to (a) but the molecule returns to its original state.

which is normally a much weaker effect than if the decay occured via vibrational modes of the molecule alone. Bath vibrations are generally very low in energy and hence population relaxation through purely bath vibrations is extremely rare and usually occurs through multiphonon processes. Vibrational relaxation to low energy modes of the surrounding molecules can occur through coupling to low frequency translation and rotational modes of molecules in the liquid.

Higher order processes that result in population relaxation, and hence affect the population lifetime, can also occur, including multiphonon relaxation (see figure 4.10) into very low energy modes of the surrounding molecules, particularly the substrate. Multiphonon decay can become a dominant mechanism for population relaxation if a single isolated vibration exists that has a much larger energy than the low frequency continuum of the bath. This is often the case with diatomic molecules which results in very long relaxation lifetimes (for example $N_{2}$ where $\left.T_{1} \sim 1 \mathrm{~s}[106,107]\right)$, but is negligible for the polyatomic molecules that we consider.

Figure 4.9(b), on the other hand, refers to an elastic process in which the molecule remains in the same mode before and after the interaction [108]. Because the intermediate states can be short lived, there is no requirement for $\omega=\omega_{1}+\omega_{2}$ and one or both of the intermediate states can be larger in energy than the original state. However, because the molecule returns to its original state, it does not contribute to $T_{1}$ but instead contributes to the broadening of the Raman peak via $T_{2}$. This contribution to $T_{2}$ occurs because the excited vibration is anharmonically interacting with other modes resulting in variations in the phase of the vibration with time. 


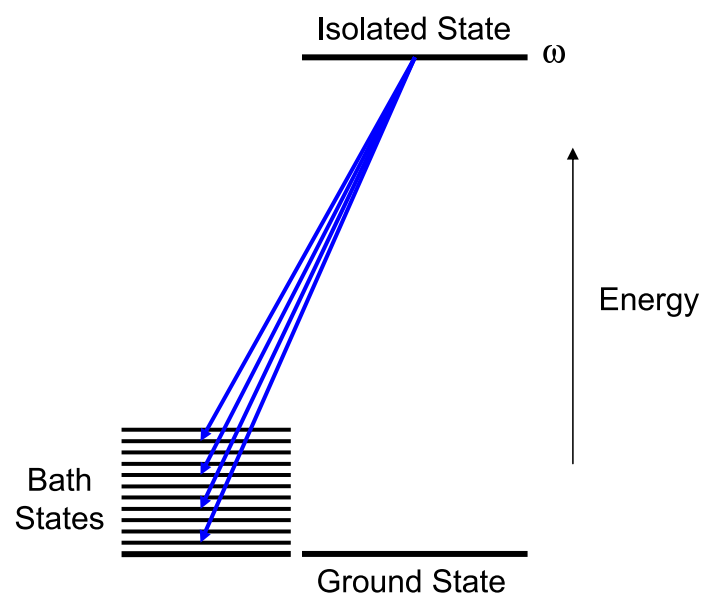

Figure 4.10: Diagram showing multiphonon relaxation of an isolated state which can only decay via the bath.

If a mode of frequency $\omega$ is anharmonically coupled to another mode, $\omega_{1}$, of less energy, determining whether this interaction has a contribution to $T_{1}$ or $T_{2}$ will depend on the existence of a second mode, $\omega_{2}$, that is also anharmonically coupled to $\omega$ satisfying $\omega=\omega_{1}+\omega_{2}$. If such a mode does exist then the vibration will be able to decay through $\omega_{1}$ and $\omega_{2}$ and hence be dominated by $T_{1}$. If such a mode does not exist then the interaction can still be dominated by $T_{1}$ if there is a vibrational state of energy $\omega_{2}$ within the bath that can assist the decay. However, if $\omega$ is very weakly coupled to the bath state then dephasing may still dominate. For a completely isolated molecule with no bath interaction, an absence of the second mode will result in the interaction being completely dominated by dephasing. For a vibration that is anharmonically coupled to a mode of higher frequency, an interaction between them can only result in dephasing and this interaction can only contribute to $T_{2}$.

Accordingly, determining whether the lifetime of a vibration is dominated by dephasing or population relaxation will depend on the existence of a vibration of less energy that is anharmonically coupled to the original mode. If no such mode exists then the vibration will be dominated by $T_{2}$. If such a mode does exist, determining whether $T_{1}$ or $T_{2}$ dominates will depend on the existence of a second state (either a vibrational state of the molecule or from the bath) satisfying $\omega=\omega_{1}+\omega_{2}$. Hence, in order to characterise each of the Raman active modes of a molecule as dephasing dominated or population relaxation dominated, one must consider these key points: $(i)$ the availability of modes 
in the vibrational density of state (VDOS), (ii) the strength of the coupling between each of the vibrational modes, and (iii) the presence and nature of interactions with the bath.

\subsubsection{Vibrational Density of States}

In order to understand how vibrational modes anharmonically couple together, an understanding of the VDOS is first required. Most SERS analytes tend to be relatively large polyatomic molecules and hence VDOS calculations can be difficult and time consuming. Depending on the level of accuracy required, there are several methods for calculating the VDOS, one of which is DFT [22]. Due to the large memory and processing requirements, it is only recently that DFT has been utilised for common SERS probes.

The VDOS for three common SERS probes (a Benzotriazole dye (BTZ2) [109], NB and RH6G) are shown in figure 4.11 calculated via DFT (for BTZ2) [110] or force field MM++ (for NB and RH6G) [111]. Each of these SERS probes can be approximated as having a VDOS that is relatively flat in the region $\sim 0-1650 \mathrm{~cm}^{-1}$, followed by a silent region in which there is little or no vibrational modes, and then a small region at $\sim 3000 \mathrm{~cm}^{-1}$ corresponding to hydrogen stretching vibrational modes. These characteristics are summarized in figure 4.11(d).

Purely from an understanding of the density of states of typical SERS analytes, we can make the qualitative argument that the high energy modes (close to $1650 \mathrm{~cm}^{-1}$ ) will strongly interact with vibrations at low energies. However, which interactions contribute to the relaxation lifetime or the dephasing lifetime will depend on whether there is a suitable pair of modes, either within the molecule itself or a combination of the molecule and the bath, that will allow the efficient decay of the vibration. If there are many such interactions, $T_{1}$ will likely dominate the peak width. As the mode energy is reduced, there are fewer possible interactions that will result in the decay of the vibration. It is therefore more likely that $T_{2}$ will become the dominant mechanism for the low energy vibrations. There will, however, be cases in which even a large energy mode will be dominated by $T_{2}$, when the structure of the vibration results in a very weak interaction with the other modes. 


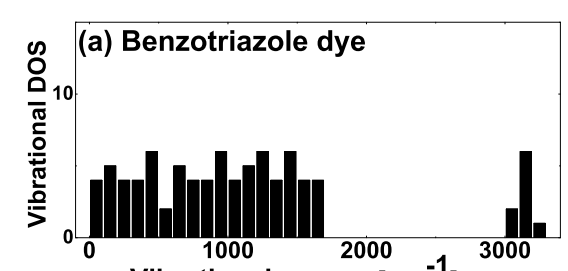

Vibrational energy $\left[\mathrm{cm}^{-1}\right]$

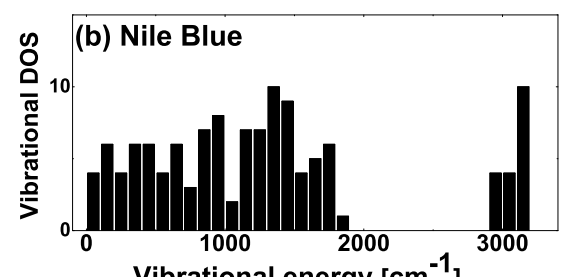

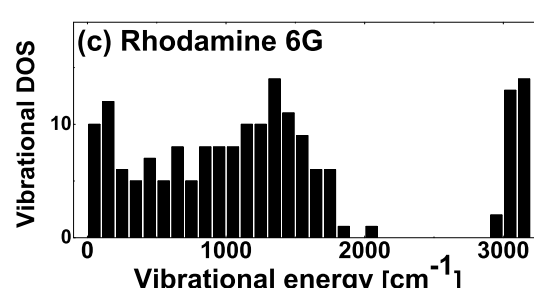

Vibrational energy $\left[\mathrm{cm}^{-1}\right]$

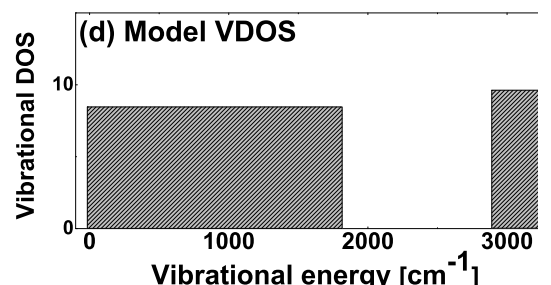

Figure 4.11: Vibrational density of states (VDOS) of three common SERS probes calculated using different methods: (a) BTZ2 [109] obtained by DFT, (b) NB and (c) RH6G calculated with the MM+ molecular mechanics force field model [111]. The overall main characteristics of most VDOS of interest are summarized in (d); i.e. a relatively flat and continuous VDOS up to $\sim 1650 \mathrm{~cm}^{-1}$ followed by isolated hydrogen stretching modes at high frequencies.

\subsubsection{Bath Interactions}

The question of whether there is a downward relaxation path that a vibration can decay through requires an understanding of the interaction between the vibration and the bath. There are no general rules for measuring the coupling strength between bath and vibration. However, by looking at specific examples, we may be able to obtain a reasonable understanding that is applicable for most SERS experiments. Observations made in the literature $[22,112]$ suggest that there are weak interactions with the substrate which can result in small frequency shifts of the bare vibrations. For most SERS situations, the interaction with the substrate is weak enough that there is never a loss in the mechanics of the bare vibrations in the molecule. However, interactions that assist relaxation definitely exist. Despite this, because these interactions are so weak, low energy vibrations, which have limited options for population relaxation through internal processes, will likely be dominated by $T_{2}$. 


\subsubsection{Investigating the Anharmonic Coupling through the Temperature Dependence}

For any anharmonic interaction between vibrational modes (like those shown in figure 4.9), the strength of the coupling will have a dependence on the thermal occupation (Bose factors) of the secondary modes $[113,114]$ through the boson operators in the perturbation process. In terms of the interactions shown in figure 4.9, the strength of the coupling will depend on the thermal occupation, $n_{1}$ and $n_{2}$, of the vibrational states with frequencies $\omega_{1}$ and $\omega_{2}$, respectively. At low temperatures, when the average thermal energy is much less than the mode energy (i.e. $k_{B} T<<\hbar \omega$ ) for all of the secondary states, the thermal occupation is very small and can be approximated by Boltzmann factors; i.e. $n \sim \exp \left(-\hbar \omega / k_{B} T\right)$. The process in figure $4.9(\mathrm{a})$ will therefore have a contribution to the peak width, $\Gamma$, given by [113]:

$$
\Gamma \propto\left(1+n_{1}+n_{2}\right)
$$

This process is responsible for the standard down-conversion into two low energy vibrations and normally referred to as a sum process [113]. Equation 4.24 has been extended to higher order multiphonon decays as shown in references [114116] but they are much weaker processes. The temperature dependence to the peak width due to population relaxation will therefore be given by an Arrhenius law:

$$
\Gamma=\Gamma_{0}+\Delta \Gamma \exp \left(\frac{-\hbar \Omega}{k_{B} T}\right) .
$$

where $\Omega$ is dependent on the available decay processes. I will return to the physical interpretation of $\Omega$.

The effect of the dephasing on the broadening due to the temperature dependence, on the other hand, is much more difficult to model because it depends on a higher order perturbation process and, therefore, more details about the VDOS and the anharmonic coupling matrix elements are required. Models for the temperature dependence of dephasing can be found in the literature, including phenomenological models that have applications for coupled harmonic oscillators, electronic states, two level systems etc. [117-121]. These models suggest that at low temperatures, and for two excitations that are closely localized in energy, the broadening will always recover an Arrhenius law for the temperature dependence [118], independent of whether the interaction is dominated by 
dephasing or population relaxation. This trend in the temperature dependence has been concluded from many different perturbative schemes [118, 122,123]. I will therefore assume that the temperature dependence of the broadening will follow an Arrhenius law (as shown in equation 4.25) which will be dependent on the most coupled-to vibration of the mode in question, independent on which lifetime dominates the peak width [124].

There are three possibilities for $\hbar \Omega$, depending on the specifics of the couplings for that mode:

- If there are limited relaxation channels then $\hbar \Omega$ will correspond to the vibrational energy that is the main contribution to dephasing, $T_{2}$.

- If there is one relaxation channel then $\hbar \Omega$ will correspond to the lowest vibrational energy that the vibration is decaying through. This is because the Boltzmann factor of the vibration with the lowest energy will dominate in the temperature dependence.

- If there are many relaxation channels that are competing with each other, then $\hbar \Omega$ will correspond to an average interaction energy which will depend on the VDOS and also the coupling strength between the vibrations.

From this point $\hbar \Omega$ will be referred to as the activation energy to be consistent with other activation phenomena that depend on the presence of a "barrier" and follow an Arrhenius law. From equation 4.25 it is obvious that a plot of $\ln \left(\Gamma-\Gamma_{0}\right)$ against $1 / T$ will have a slope that is proportional to the activation energy. i.e:

$$
\ln \left(\Gamma-\Gamma_{0}\right)=\frac{-\hbar \Omega}{k_{B} T}+\ln (\Delta \Gamma)
$$

In section 4.4.2 it was concluded that a single vibrational mode interacts with a quasi-continuum of modes. Hence, in general, $\hbar \Omega$ will not correspond to a specific vibration but instead an average of the main relaxation channels. However, the estimation of $\Omega$ from the temperature dependence of the broadening will give an approximation of the main couplings affecting the mode $\omega$ :

- If $\Omega<\omega$, the mode must be mainly coupled to vibrations of less energy which could allow population relaxation of $\omega$. Consequently, for the polyatomic molecules that are common to SERS probes, this case will likely correspond to a mode that is $T_{1}$-dominated due to the larger number of 

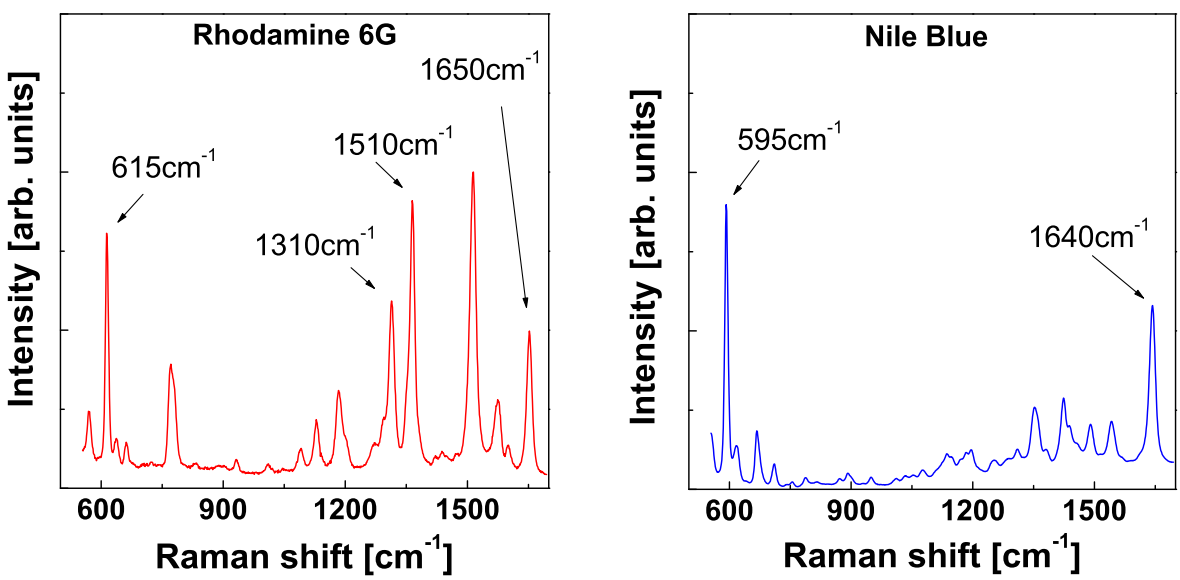

Figure 4.12: $\quad S E R S$ spectra for $N B$ and RH6G. The modes monitored are the $595 \mathrm{~cm}^{-1}$ and $1640 \mathrm{~cm}^{-1}$ of $N B$ and $615 \mathrm{~cm}^{-1}, 1310 \mathrm{~cm}^{-1}, 1510 \mathrm{~cm}^{-1}$, and $1650 \mathrm{~cm}^{-1}$ of RH6G.

vibrations in the VDOS. In general, higher energy modes will have more possibilities for population relaxation via two or more phonons.

- If $\Omega \sim \omega$, it is impossible to decide which contribution dominates the peak broadening.

- If $\Omega>\omega$, coupling to higher energy modes must be the dominant contribution and, hence, population relaxation through downward conversion is much more difficult. These modes must therefore be dominated by $T_{2}$ and the width of these peaks cannot be used as an estimation for the population lifetime.

Other effects such as a temperature dependence of the peak width due to inhomogeneous broadening is not expected to play a significant role, at least not as much as $T_{1}$ and $T_{2}$.

\subsubsection{Experimental Results}

Using the theory outlined in section 4.4.4, we will estimate the dominant mechanism (dephasing or population relaxation) of the broadening of the peak widths for two common SERS probes, NB and RH6G. The Raman modes that are characterised as $T_{1}$-like (coupled to modes of lower energy) are the best candidates for measuring the pumping cross-section because their peak widths are a good approximation of the population lifetime. 

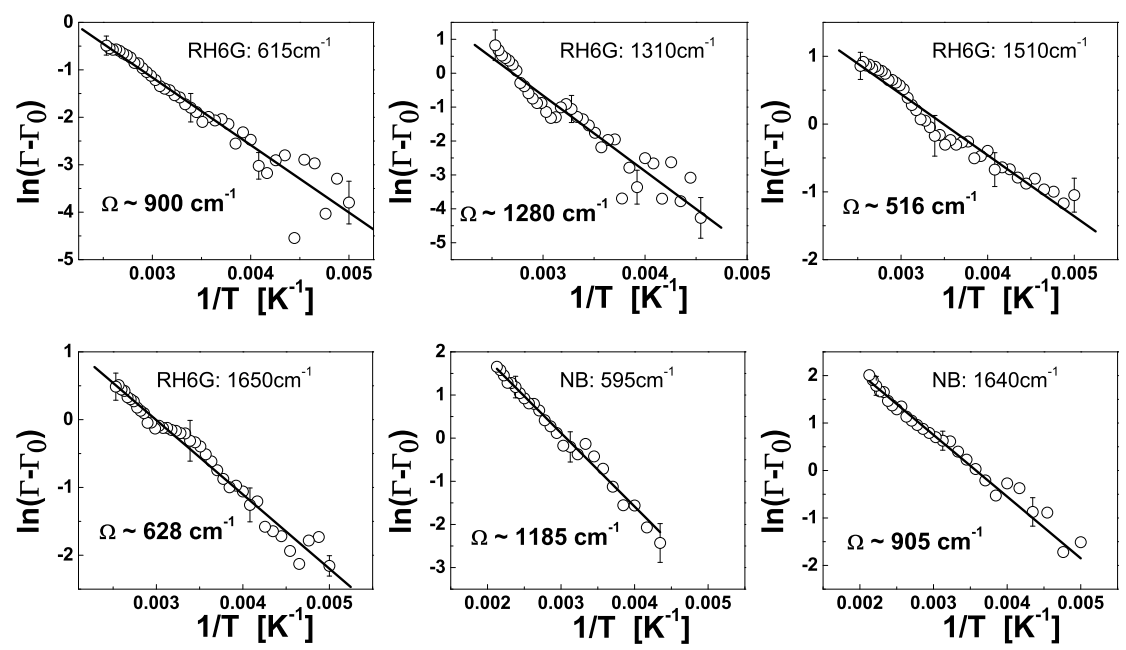

Figure 4.13: Temperature dependence of $\ln \left(\Gamma-\Gamma_{0}\right)$ as a function of $1 / T$ for the four modes of RH6G and the two modes of NB shown in figure 4.12. Included are the linear fits and the activation energy derived from the slope. The results are summarized in table 4.1.

A dry sample consisting of colloids, $10 \mathrm{mM} \mathrm{KCl}$ and $1 \mu \mathrm{M}$ of the SERS probe, and completely dried was mounted on the sample holder of the U1000 system (described in section 4.3.1). The sample was placed within the vacuum sealed chamber and cooled using the close cycle helium cryostat. An excitation wavelength of $676 \mathrm{~nm}$ was created using the krypton laser. Because accurate values for the peak width, height and frequency were required, the peaks were fitted using Voigt fits $[7,125]$. The power density was kept relatively low $(3.53 \times$ $10^{8} \mathrm{~W} / \mathrm{m}^{2}$ ) to reduce the effect of photobleaching and heating. The laser was focused on a region with a diameter of approximately $12 \mu \mathrm{m}$.

The Raman spectra for the two SERS probes are shown in figure 4.12. The Raman modes which were monitored are labelled, namely the $615 \mathrm{~cm}^{-1}$, $1310 \mathrm{~cm}^{-1}, 1510 \mathrm{~cm}^{-1}$ and $1650 \mathrm{~cm}^{-1}$ of RH6G, and the $595 \mathrm{~cm}^{-1}$ and $1640 \mathrm{~cm}^{-1}$ of NB.

The peak width for each of these modes was measured as a function of temperature and plots were constructed of $\ln \left(\Gamma-\Gamma_{0}\right)$ against $1 / T$ (see figure 4.13) in order to obtain the activation energy from the linear fit. The results are summarized in table 4.1. As was expected, the two largest energy modes of RH6G and the largest energy mode of NB all have an $\Omega$ which is less than $\omega$, hence, these modes must be mainly coupled to lower energy vibrations. We 


\begin{tabular}{|c|c|c|c|}
\hline \hline Compound & mode, $\omega\left[\mathrm{cm}^{-1}\right]$ & activation energy, $\Omega\left[\mathrm{cm}^{-1}\right]$ & mode type \\
\hline RH6G & 615 & $\sim 900$ & $T_{2}$-like \\
\hline RH6G & 1310 & $\sim 1280$ & $T_{2} / T_{1}$-like \\
\hline RH6G & 1510 & $\sim 516$ & $T_{1}$-like \\
\hline RH6G & 1650 & $\sim 628$ & $T_{1}$-like \\
\hline NB & 595 & $\sim 1185$ & $T_{2}$-like \\
\hline NB & 1640 & $\sim 905$ & $T_{1}$-like \\
\hline \hline
\end{tabular}

Table 4.1: Anharmonic classification of main SERS active modes in RH6G and NB.

therefore classify these modes as $T_{1}$-like.

The $1310 \mathrm{~cm}^{-1}$ mode of RH6G, on the other hand, has an activation energy that is very similar to the mode energy. Deciding whether this mode is dominated by population relaxation or dephasing is difficult. It is more likely that neither mechanism dominates and each coupled-to mode contributes both to $T_{1}$ and $T_{2}$. This mode is therefore undefined and cannot be referred to as either $T_{1}$ or $T_{2}$-like.

The $615 \mathrm{~cm}^{-1}$ of RH6G and $595 \mathrm{~cm}^{-1}$ of NB both have an $\Omega$ that is much larger than $\omega$. These modes must therefore be mainly coupled to modes of higher energy and the main interactions can only contribute to dephasing. We can therefore confidently classify these modes as $T_{2}$-like.

In addition, it is possible to measure the activation energy from the frequency shift, which is also dependent on an Arrhenius law. As a result, a plot of $\ln \left(\omega-\omega_{0}\right)$ against $1 / T$ will similarly reveal $\Omega$. However, measuring the temperature dependence of the broadening is often easier, especially if the spectrometer must be moved to different spectral regions as we can ignore the systematic uncertainty resulting from the backlash of the spectrometer. Despite this, if the Raman modes are measured in a fixed window, and at a high enough resolution, it is possible to measure the activation energy from the temperature dependence of both the broadening and the frequency shift. Figure 4.14 presents $\Omega$ estimations from both the frequency shift and the broadening for the $1650 \mathrm{~cm}^{-1}$ of RH6G.

These findings show that the high energy modes are mainly coupled to low energy vibrations and hence are likely dominated by population relaxation (at least in the case of $\mathrm{NB}$ and $\mathrm{RH} 6 \mathrm{G}$ ). We have also seen that low energy vibrations are dominated by dephasing and hence population lifetime estimates for these modes cannot be calculated from their peak-widths. However, there may be exceptions, for example when there is strong coupling to the substrate and low 


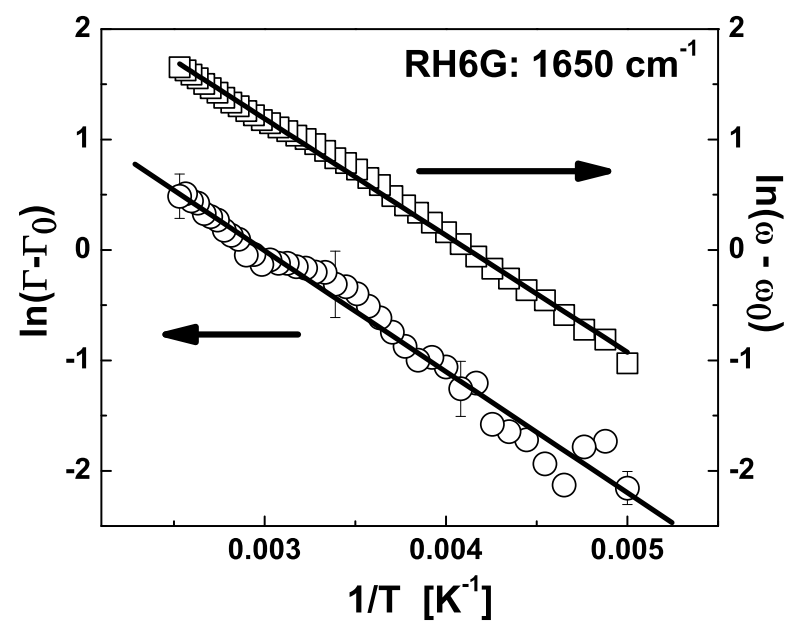

Figure 4.14: Broadening $\left(\ln \left(\Gamma-\Gamma_{0}\right)\right)$ and frequency $\left(\ln \left(\omega-\omega_{0}\right)\right)$ dependence of the $1650 \mathrm{~cm}^{-1}$ mode of RH6G as a function of $1 / T$, both showing the same slope $\propto \hbar \Omega$ (activation energy).

frequency vibrations are dominated by bath interactions.

\subsection{The Plasmon Dispersion and Two Laser Pump- ing}

Two-laser vibrational pumping is an experimental technique for measuring the SERS enhancement over a large number of excitation wavelengths without having to calibrate the response of the system in that range. This is achieved by adding a second laser, called the probe laser, which has the role of fixing the scattering wavelength. The vibrational population is initially created by a pumping laser and the relaxation of the population is monitored from the antiStokes signal relative to the probe laser. The anti-Stokes intensity will therefore provide information about the coupling between the plasmon resonance distribution created with each laser. The equations outlined in section 4.2 .1 will be extended to the case of a double laser excitation with as little repetition as possible. 


\subsubsection{The Theory of Two Laser Pumping}

A basic two-laser pumping experiment will consist of two lasers (a pump and probe), that are combined using a beam splitter, and a dry sample contained within a cryostat. Furthermore, the probe laser spot must be located within the pump laser spot after it has been focused on the sample. We are therefore probing a sub-domain of the total excited region of the substrate. Using the cryostat, the sample is cooled to a thermal region in which vibrational pumping is the dominant mechanism of population creation. The vibrational population created by the pump through Stokes processes at the pump wavelength $\left(\lambda_{\mathrm{pu}}\right)$ is monitored as an increase in the anti-Stokes signal at the probe wavelength $\left(\lambda_{\mathrm{pr}}\right)$. The magnitude of the increase will therefore contain information on how well the probe can couple to molecules that have been excited by the pump. Taking low temperature measurements (in the vibrational pumping regime) ensures that only those molecules that receive a sufficiently large enhancement at the pump wavelength will increase the anti-Stokes signal relative to the probe. If measurements were taken in the thermally excited regime, the increase in the anti-Stokes signal would be extremely small and easily confused with heating. Furthermore, every molecule in the sample will have a similar population, not just those located at hot-spots.

In two-laser pumping, we are concerned with anti-Stokes events due to the probe, where the original vibration was created via a Stokes process with the pump. However, there is also a contribution to the anti-Stokes signal where the original vibration was created by the probe, which we call self-pumping. These processes can be removed by subtracting the anti-Stokes signal with only the probe active from the signal with both lasers active.

For a Raman active mode of a single molecule, the population in the pumping regime is given by:

$$
n=\frac{\tau \sigma_{p}^{\mathrm{pu}} P^{\mathrm{pu}}}{\hbar \omega^{\mathrm{pu}}}+\frac{\tau \sigma_{p}^{\mathrm{pr}} P^{\mathrm{pr}}}{\hbar \omega^{\mathrm{pr}}}
$$

where $\sigma_{p}^{\mathrm{pu}, \mathrm{pr}}, P^{\mathrm{pu}, \mathrm{pr}}$ are the total Stokes cross-sections $\left(\sigma_{S}^{T}\right.$ has been substituted for $\sigma_{p}$ to simplify the equations in this section) and power densities at the pump and probe wavelengths, respectively. This is the same as equation 4.5 but with two lasers creating the population and ignoring the thermally excited population.

If there are $N$ molecules in the region defined by the probe spot (which is 
already contained within the pump spot), the intensity of the anti-Stokes signal of the probe is:

$$
I_{a S}=N \tau\left[\frac{\left\langle\sigma_{p}^{\mathrm{pu}} d \sigma_{a S}^{\mathrm{pr}} / d \Omega\right\rangle P^{\mathrm{pu}}}{\hbar \omega^{\mathrm{pu}}}+\frac{\left\langle\sigma_{p}^{\mathrm{pr}} d \sigma_{a S}^{\mathrm{pr}} / d \Omega\right\rangle P^{\mathrm{pr}}}{\hbar \omega^{\mathrm{pr}}}\right] P^{\mathrm{pr}},
$$

where the differential cross-sections refer to radiative Stokes or anti-Stokes scattering (the superscript $R$ has been left out to simplify the equations). This equation consists of both a quadratic power dependence of the probe (the selfpumping term seen in single laser pumping), and a linear power dependence of the pump, the "cross-talking" term, which is a result of the interaction between the two lasers. The anti-Stokes intensity with only the probe active is given by:

$$
I_{a S}^{0}=N \tau \frac{\left\langle\sigma_{p}^{\mathrm{pr}} d \sigma_{a S}^{\mathrm{pr}} / d \Omega\right\rangle P^{\mathrm{pr}}}{\hbar \omega^{\mathrm{pr}}} P^{\mathrm{pr}} .
$$

Taking the difference in the two intensities gives:

$$
\Delta I_{a S}=N \tau \frac{\left\langle\sigma_{p}^{\mathrm{pu}} d \sigma_{a S}^{\mathrm{pr}} / d \Omega\right\rangle P^{\mathrm{pu}}}{\hbar \omega^{\mathrm{pu}}} P^{\mathrm{pr}} .
$$

Because the number of molecules is unknown, it is often convenient to normalize this with the Stokes intensity of the probe:

$$
I_{S}^{\mathrm{pr}}=N\left\langle d \sigma_{S}^{\mathrm{pr}} / d \Omega\right\rangle P^{\mathrm{pr}} .
$$

Thus, taking the ratio of equation 4.30 and 4.31 we obtain:

$$
\frac{\Delta I_{a S}}{I_{S}^{\mathrm{pr}}} \propto \frac{\left\langle\sigma_{p}^{\mathrm{pu}} d \sigma_{a S}^{\mathrm{pr}} / d \Omega\right\rangle}{\left\langle d \sigma_{S}^{\mathrm{pr}} / d \Omega\right\rangle} P^{\mathrm{pu}}
$$

The term $\left\langle\sigma_{p}^{\mathrm{pu}} d \sigma_{a S}^{\mathrm{pr}} / d \Omega\right\rangle$ is a measure of how well the probing laser can couple to molecules that have been excited by the pump, and hence this is a measure of the wavelength dependence of the spatial distribution of plasmon resonances. It is important to note that it is not a measure of the dispersion of the plasmon resonance for a single molecule but an average over all molecules that are contributing to the signal. Furthermore, a single value of $\left\langle\sigma_{p}^{\mathrm{pu}} d \sigma_{a S}^{\mathrm{pr}} / d \Omega\right\rangle$ does not contain much physical information as the two cross-sections cannot be de-coupled. However, some insight into the distribution of plasmon resonances can be gained by observing how it varies with pump or probe wavelength. Figure 4.15 shows an example of the distribution of hot-spots at two wavelengths for 


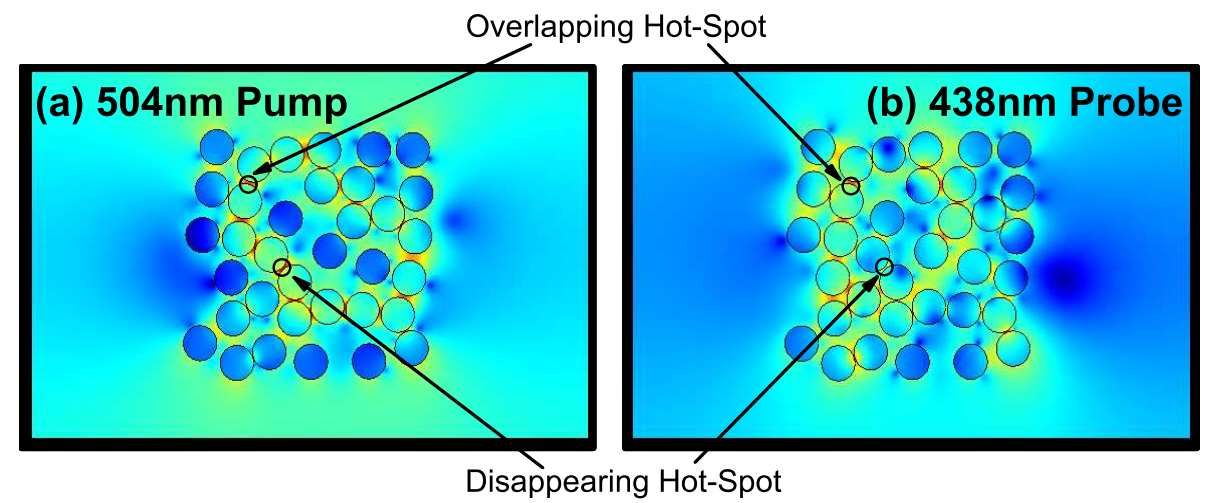

Figure 4.15: The two dimensional simulation performed in section 3.3 .3 but with an excitation wavelength of (a) $504 \mathrm{~nm}$ and (b) $438 \mathrm{~nm}$. Two gap regions are pointed out, one in which the local field enhancement is significant at both wavelengths (the overlapping hot-spot) and one in which the local field enhancement is only large for the pumping wavelength (the disappearing hot-spot).

the two dimensional simulation in section 3.3.3. Those molecules that receive a large local field enhancement in figure 4.15(a) will have the largest average population. However, these molecules will only be observed as an increase in the anti-Stokes intensity relative to the probe wavelength if they also receive a large local field enhancement in figure 4.15(b). Let us consider a single molecule that is located in the overlapping hot-spot. This molecule will receive a large enhancement from the pump and the probe. The product $\sigma_{p}^{\mathrm{pu}} d \sigma_{a S}^{\mathrm{pr}} / d \Omega$ will therefore be relatively large and the molecule will contribute significantly to $\Delta I_{a S}$. If we now consider a molecule in the location of the disappearing hotspot, this molecule will have a significant population due to the pumping laser. However, the enhancement from the probe is relatively weak and hence the molecule will not contribute much to $\Delta I_{a S}$.

Equation 4.32 demonstrates that $\Delta I_{a S} / I_{S}^{\mathrm{pr}}$ has a linear dependence on $P^{\mathrm{pu}}$ of which $\left\langle\sigma_{p}^{\mathrm{pu}} d \sigma_{a S}^{\mathrm{pr}} / d \Omega\right\rangle$ can be calculated directly from the slope (if $\left\langle\left(d \sigma_{S}^{\mathrm{pr}} / d \Omega\right)\right\rangle$ is known). In order to observe the coupling between the pump and probe laser in addition to the self-pumping contribution, a low probe laser power must be used. This becomes more important for large differences in pump-probe wavelengths as the coupling may be very weak. 


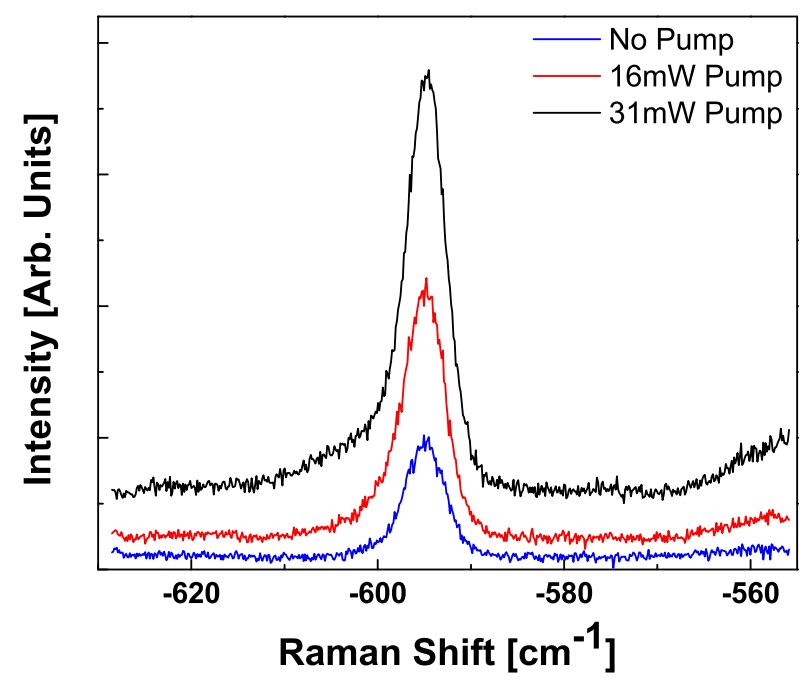

Figure 4.16: Two-laser pumping spectra for the $595 \mathrm{~cm}^{-1}$ anti-Stokes peak of NB relative to the $633 \mathrm{~nm}$ probe. Measurements are taken with a $647 \mathrm{~nm}$ pump at $0 \mathrm{~mW}$, $16 \mathrm{~mW}$ and $31 \mathrm{~mW}$ at a temperature of $10 \mathrm{~K}$. This figure is adapted from figure 12 in reference [92].

\subsubsection{Experimental Results}

For this experiment, NB was chosen for the SERS probe as it has an extremely large cross-section in the appropriate wavelength regions [126]. This is important as the coupling between the probe and pump may be quite weak and the increase in intensity may be difficult to measure, especially for the low probe powers needed. The sample preparation was the same as described in section 4.3.3; Ag colloids, $10 \mathrm{mM} \mathrm{KCl}$, and $\mathrm{NB}$ solution at a final concentration of $1 \mu \mathrm{M}$, dried on a silicon wafer. The sample is mounted on the U1000 Jobin-Yvon spectrometer (see section 4.3.1). Due to the large and low powers required for the pump and probe, respectively, the $676 \mathrm{~nm}$ and $647 \mathrm{~nm}$ emission lines of the Krypton laser were used as pumps, and the $514 \mathrm{~nm}$ line of the Argon laser and the $633 \mathrm{~nm}$ line of the Helium-Neon laser were used as probes. Having the probe at a shorter wavelength than the pump has the advantage that the anti-Stokes peak of the probe is in the emission-free region of both lasers and the signal is not drowned out by fluorescence.

Figure 4.16 shows the variation of the Raman signal for the $595 \mathrm{~cm}^{-1}$ antiStokes peak of NB with respect to the $633 \mathrm{~nm}$ probe, with a power of $3 \mathrm{~mW}$, when varying the $647 \mathrm{~nm}$ pump power. When the pump is inactive, we see 

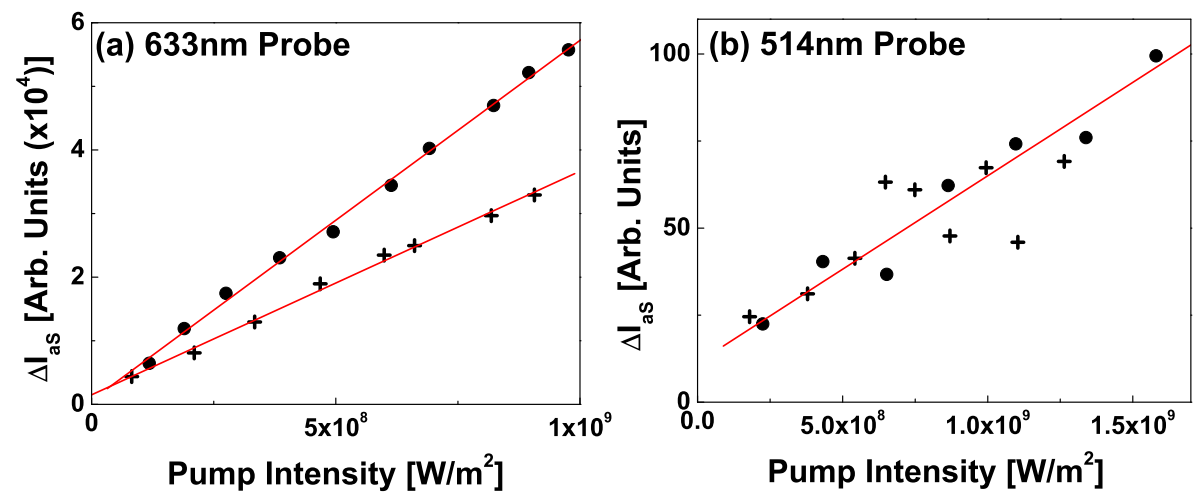

Figure 4.17: Plots of the increase in the $595 \mathrm{~cm}^{-1}$ anti-Stokes intensity $\left(\Delta I_{a S}\right.$ in equation 4.30) of $\mathrm{NB}$ with respect to the (a) $633 \mathrm{~nm}$ and (b) $514 \mathrm{~nm}$ probe with pumping wavelengths, $647 \mathrm{~nm}$ (circle points) and $676 \mathrm{~nm}$ (cross points). The y-scale in (a) is a factor of $10^{4}$ larger than for (b). This figure is adapted from figure 13 in reference [92].

the SERS intensity resulting from self pumping of the Raman mode by the probe. As the pump power increases, so does the vibrational population and, consequently, the anti-Stokes intensity increases. The increase in the intensity follows a linear relationship with pump power as predicted by equation 4.30.

The increase in anti-Stokes intensity $\left(\Delta I_{a S}\right)$ as a function of pumping power is shown in figure 4.17 for both the $633 \mathrm{~nm}$ and the $514 \mathrm{~nm}$ probes. Each are pumped with the $647 \mathrm{~nm}$ and $676 \mathrm{~nm}$ lines. The laser power densities were estimated by measuring the scattering width as described in appendix B.

For the $633 \mathrm{~nm}$ probe (figure 4.17(a)), the slope (which is proportional to the plasmon dispersion) for the $647 \mathrm{~nm}$ pump is greater than the $676 \mathrm{~nm}$ pump. This tells us that molecules that benefit from the plasmon resonance at $633 \mathrm{~nm}$ are more likely to also benefit from the resonance at $647 \mathrm{~nm}$ than at $676 \mathrm{~nm}$. We are therefore seeing a "detuning" of the local field as we move away from the probe wavelength. To a good approximation, the only molecules that are contributing to $\Delta I_{a S}$ are those molecules where there is a significantly large hotspot at both the pumping and probing wavelengths. The number of molecules, however, that meet this criteria will decrease as the difference in the wavelengths becomes larger (this was shown in section 3.3.3).

If the probe laser wavelength is reduced to $514 \mathrm{~nm}$ (see figure $4.17(\mathrm{~b})$ ), the slope of the linear fit is approximately 600 times less than with the $633 \mathrm{~nm}$ probe. This tells us that, on average, molecules excited by the pump are only weakly coupled to the probe, and hence the local-field for the $514 \mathrm{~nm}$ excitation 


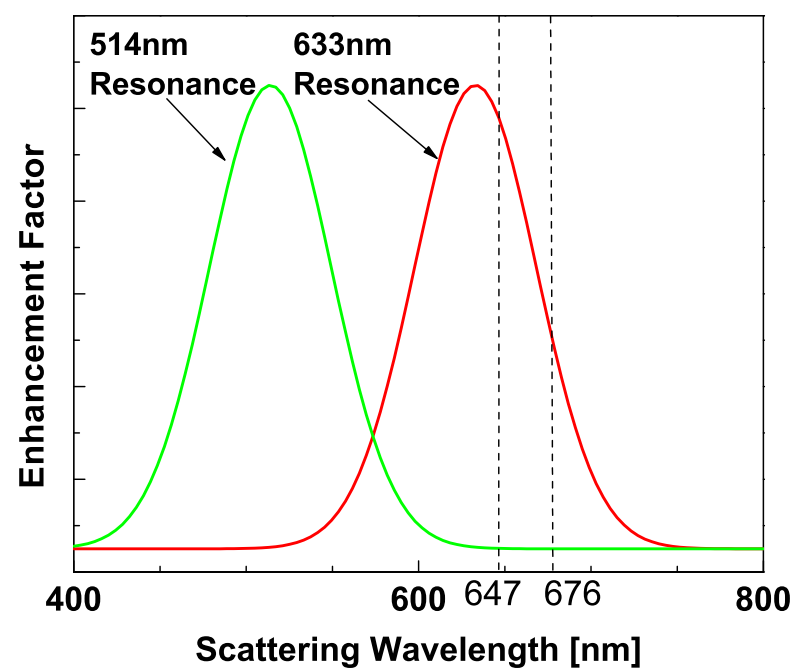

Figure 4.18: A simplified model of the enhancement factor distribution for two molecules; one located at a $633 \mathrm{~nm}$ excitation hot-spot and one located at a $514 \mathrm{~nm}$ excitation hot-spot. In general, the enhancement spectra will not have the same shape but this figure gives a rough idea of what is occurring in figure 4.1\%. The two vertical dashed lines correspond to the $647 \mathrm{~nm}$ and $676 \mathrm{~nm}$ pump.

is very different to the local-field at both $647 \mathrm{~nm}$ and $676 \mathrm{~nm}$. Furthermore, in figure 4.17(a), the amount of detuning of the local field is obvious as we change from the $647 \mathrm{~nm}$ pump to the $676 \mathrm{~nm}$ pump, but this is not the case for the $514 \mathrm{~nm}$ probe in which the two pumps show very similar results. One possible explanation is the distribution of enhancement factors for different molecules. In section 3.2.1 it was explained that the enhancement factor depends on both the incident and scattered wavelengths. For the case of two-laser pumping, however, the increase in the anti-Stokes signal from a single molecule depends on the enhancement factor at both the pump and probe wavelengths. Figure 4.18 shows an idealised case of the enhancement factor distribution for two molecules, one at a $633 \mathrm{~nm}$ excitation hot-spot and one at a $514 \mathrm{~nm}$ excitation hot-spot. For the $633 \mathrm{~nm}$ molecule, there is a significant difference between the enhancement factor at the two pumping laser wavelengths as the width of the resonance is larger than the difference in the pump and probe wavelengths. This is not the case for the $514 \mathrm{~nm}$ molecule as we are observing the tail of the enhancement factor distribution where there is very little difference between the two pumping wavelengths.

It may be possible to explain the difference in the two slopes for figure 4.17(a) 
as a variation in the Raman cross-section at the pump wavelengths. However, if this were the case, there should also be evidence of this effect in figure 4.17(b). We can therefore conclude that the slope is consistent with the dispersion in the plasmon resonance distribution.

\subsubsection{Limitations and Applications for Two Laser Pump- ing}

In principle there are two effects in which two constant wave (CW) lasers can contribute to the same Raman signal; through two-laser pumping, in which both lasers create a vibrational population that can be observed from the antiStokes peak relative to the probe, and via laser heating of the sample by the pump, which will also lead to an increase in the anti-Stokes signal of the probe. The change in the anti-Stokes intensity due to these two mechanisms will be different, however, as laser heating will increase the vibrational population of all the molecules in the sample, while vibrational pumping by the pump laser will mainly increase the vibrational population in molecules that experience a large plasmon resonance and are Raman active. Furthermore, laser heating is only important in the thermally dominated regime where the population is dominated by thermal excitation. The coupling between the two lasers, in this case, is via the absorption cross section and not Raman (as in the two laser pumping case). As two-laser pumping experiments are always performed in the low temperature pumping regime, the increase in the population (if any) due to laser heating is negligible and this mechanism can be ignored.

Like any technique, two-laser pumping has advantages and disadvantages. The equipment required for low temperature experiments $(\sim 10 \mathrm{~K})$ and also to align two lasers (such that one laser spot is contained within the other) can be difficult to obtain. It is also important that the pump is always at a longer wavelength than the probe so that the Raman peak being measured is not in the emission region of the pump and drowned out by fluorescence. However, if a high power tunable laser is available, then it is possible to measure the dispersion of the resonance (at least for wavelengths longer than the probe) using the tunable laser as a pump without having to calibrate the response of the system [127]. This is because we can fix the scattering wavelength (which corresponds to an anti-Stokes Raman peak of the probe) whilst the excitation wavelength (corresponding to the Stokes peak for the same mode) is varied. Another benefit of having a fixed scattering wavelength is that it is possible 
to measure the dispersion in regions that cannot be directly observed with the spectrometer (for example, in the near-IR range) if the appropriate pumping lasers are available. Furthermore, two-laser pumping allows measurements of the local field dispersion using systems that operate with notch filters in which the scattering wavelength is restricted by which notch filters are available. It is impossible to obtain detailed dispersion measurements using single laser techniques for these systems.

Even with the difficulties associated with performing experiments of this type, two-laser pumping gives us the rare opportunity to observe an interaction between two laser excitations. Other techniques involving absorption (femtosecond pump and probe spectroscopy for example) or coherent anti-Stokes Raman scattering $[128,129]$ also have this property, but two laser pumping is one of the few cases in which the interaction is achieved via the incoherent vibrational population.

Through two-laser pumping we have achieved a higher level of understanding of the vibrational pumping technique. Several avenues of advancement present themselves through this technique, including the use of multiple dyes to compare the spatial distribution of molecules (i.e. whether different types of dyes populate similar regions of the sample). Another interesting question is the effect that having a pump and probe of different polarizations or phases (when both lasers have the same wavelength) will have. These are possibilities that could be explored in the future.

\subsection{Conclusion}

In this chapter two aspects of vibrational pumping were investigated. Firstly, measurements of anharmonic coupling between modes were performed, and secondly, an application of vibrational pumping was discussed in the form of twolaser pumping. Also included was a brief introduction to vibrational pumping theory and a detailed description of the experimental equipment and sample preparation methodology.

The study of the anharmonic coupling between vibrational states provided evidence that for the analytes investigated (RH6G and NB), the higher energy modes were more likely to have peak widths that were predominantly determined by the relaxation lifetime. It is not possible, however, to assume that this is always the case as there may be circumstances where the structure of the 
vibration only allows for weak coupling with other modes. Ideally we would like to be able to perform the coupling measurements performed in section 4.4.5 each time a pumping experiment is carried out to confirm that the peakwidth is a good estimate of the lifetime. However, this requires high resolution measurements of each mode which can be very time consuming.

Using two-laser pumping, measurements of the dispersion in the plasmon resonance distribution, at a specific probe wavelength, could be performed by simply adjusting the pumping wavelength. This has the benefit that the response of the system does not have to be calibrated, as the scattering wavelength will remain constant and only the excitation wavelength will change. There are, however, several experimental complexities with this technique including the need for: a cryostat to reach pumping temperatures, a beam splitter to combine the pump and probing lasers, and a large number of pumping laser lines if a complete mapping of the resonance is required. One of the key drawbacks of this technique is the requirement that the probe excitation be at a higher energy than the pump excitation as only half of the resonance can be mapped. However, it does give us a unique technique for observing the interaction between two lasers.

At this stage in the thesis, the interpretation of the pumping cross-section is inconclusive. The Stokes processes that create the vibrational population within a molecule can be both radiative or non-radiative. However, only radiative scattering is observed on the Stokes side of the SERS spectrum under pumping conditions. As a result, identifying which scattering mechanism is dominating the pumping cross-section is not yet possible. This problem will be addressed in section 6.5 . 


\section{Chapter 5}

\section{Wavelet Transforms and Background Removal}

\subsection{Introduction}

This chapter was originally developed as a completely independent study in signal processing for interest in SERS. However, it turned out to be an extremely useful tool to address one of the most important topics in this thesis: the measurement of non-radiative properties in SERS. For that reason, it is included here as a preamble of the final chapter on non-radiative cross-sections. Among other things, the context of this chapter has resulted in a full paper in Applied Spectroscopy [2] and a freely available application from the website of our lab [130].

In SERS we often deal with signals that not only have a contribution from Raman scattering, but also fluorescence. Each of these processes are affected similarly by the local environment of the molecule but with slight differences. Furthermore, often we are only interested in one or the other, and rarely both. A technique for separating these two components is therefore very desirable. Fluorescence is typically continuous over all wavelengths and results in a background on which the Raman peaks are superimposed. This situation is not unique to SERS and can be generalized to many spectroscopic applications, including IR, NMR, etc ... It is often the case that many spectra need to be separated into their components (for example when performing time series or spatial mappings containing thousands of events) which is obviously impossible to do for every 
spectrum individually.

Our particular interests lie in single molecule SERS. When observing the SERS signal from a single molecule, the fluorescence background and relative intensities of the Raman modes can vary dramatically between events. This is because we are observing the dispersion of the plasmon resonance for a single metallic cluster instead of an average over many. As mentioned before, in the next chapter an investigation into how non-radiative effects modify the SEF and SERS signal for single molecules will be performed, where accurate measurements of both the SEF and SERS intensities are required. However, single molecule events are rare and large numbers of spectra (thousands to tens of thousands) need to be measured. It is therefore impossible to consider each one individually and manually measure the SEF and SERS intensities. As a result, an application that can separate the SEF and SERS signal for many spectra is needed.

Using wavelet transforms, the signal (containing noise, signal peaks and background), can be decomposed into levels which contain information on the different frequency components. For example, the highest frequency information of the signal, which is typically noise, is contained within the lowest decomposition levels. Wavelet transforms are very similar to Fourier transforms except that the signal is decomposed into wavelets, which are analogous to localised "sinusoids", instead of sine functions. As a result, wavelet transforms are better suited to localised signal events, like Raman modes, and low frequency components, like the background. Using the high decomposition level information, I will show that it is possible to reduce the signal peaks until an accurate background fit can be made. Furthermore, the algorithm described in this chapter is able to remove backgrounds from many spectra without any additional input from the user.

Three examples will be investigated with very different types of backgrounds. The first example will include a simulated spectrum with several different wavelet transform based fittings. In the second case, the background will change in overall intensity but very little in shape (and frequency). The final example will contain measurements of single molecule SERS. In all cases I will show that the algorithm proposed in this chapter obtains extremely accurate results. 


\subsection{The Theory of Wavelet Transforms}

Wavelet transforms (WTs) were first investigated in the early 80s but were not widely accepted until the important publications by Daubachies (1988) [131] and Mallat (1989) [132] looking at WTs in signal processing. Since then, there has been a large number of publications in analytical chemistry [133-140] that utilize WTs, including Raman spectroscopy [141-143].

In this section, I will give a brief theoretical overview of WTs and the advances made by Daubachies and Mallat. However, the field of WTs is extremely large and beyond the scope of this thesis. A more detailed overview can be found in literature such as [144-146].

\subsubsection{Wavelet Transforms}

The theory of WTs was first developed to overcome some of the shortcomings with Fourier transforms (FTs). Using FTs, a signal can be transformed into the frequency domain where the different frequency components of the signal can be filtered as necessary. The term "frequency" refers to the variable in the abscissa which often corresponds to "time", however, it may also correspond to other physical variables. In the case of Raman, the abscissa is the difference in energy between the scattered and incident light. The advantage of filtering the signal in the frequency domain is that many signal contributions can be distinguished by their frequency components. For example, in many spectroscopic applications, the noise contribution to the signal is typically dominant at higher frequencies. Hence, by reducing the high frequency components (using a low pass filter), and then inverse Fourier transforming the signal, we can significantly reduce the amount of noise.

The complex Fourier amplitude at a certain frequency is often defined as:

$$
F(\omega)=\frac{1}{\sqrt{2 \pi}} \int_{-\infty}^{\infty} f(x) e^{-i \omega x} d x
$$

for a continuous function $f(x)$. The Fourier amplitude is therefore a correlation between the signal and a "sinusoid" $\left(e^{-i \omega x}\right)$ of frequency $\omega$. FTs have the disadvantage that all of the information about the relationship between the signal and the abscissa is lost in the frequency domain. This can be a significant weakness for signal analysis in areas such as Raman spectroscopy, where information about the frequency components at the location of the Raman peaks is important. 

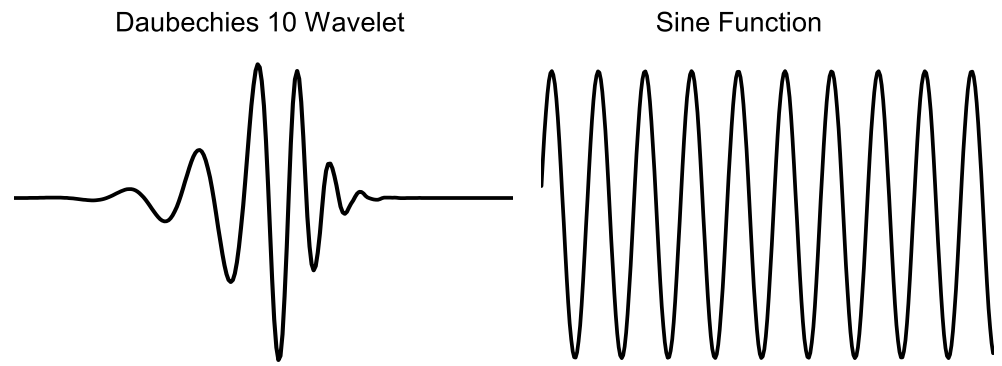

Figure 5.1: Plots of a Daubechies 10 wavelet and a sine function. The wavelet is localised with a defined width (scale) and position. The sine function extends out to infinity and hence is completely defined by its frequency.

A solution to this problem is the use of time-frequency transforms $[133,147$, 148] in which a FT is performed on sub-divisions of the full signal. It is therefore possible to obtain Fourier amplitudes that are a function of the abscissa and frequency. However, FTs become less accurate for frequency components that have a period that is similar to or larger than the size of the region in which the FT is performed. As a result, time-frequency transforms require a tradeoff between resolution in the two variables; to get the best abscissa resolution, the sub-divisions need to be small, and consequently, the FT in these regions becomes less accurate.

WTs attempt to avoid these problems by decomposing the signal into wavelets, which are analogous to localised "sinusoids" (see figure 5.1), and are non-zero in a limited domain. The wavelet coefficient is given by:

$$
W(a, b)=\int_{-\infty}^{\infty} f(x) \psi_{a, b}(x) d x,
$$

where $\psi_{a, b}(x)$ is a wavelet function with two parameters, scale, $a$, and position, $b$. This equation can be rewritten as equation 5.1 by replacing $\psi_{a, b}(x)$ with the function $e^{-i \omega x}$. Which signal processing technique is the most effective will depend on the shape of the signal. For periodic signals, with very few localised events, a FT will typically give the best results. For signals with many localised events, as seen in many spectroscopic applications, WTs will tend to be more accurate.

As in FTs, the wavelet coefficients can be filtered and reconstructed into a modified signal using: 


$$
f(x)=\int_{-\infty}^{\infty} \int_{0}^{\infty} W(a, b) \psi_{a, b}^{*}(x) d a d b,
$$

where $\psi_{a, b}^{*}(x)$ is the complex conjugate of $\psi_{a, b}(x)$.

\section{Scale and Position}

In FT, the sinusoidal function is only dependent on the frequency. Wavelets, on the other hand, are dependent on two parameters, the scale and position. Because a wavelet is localised, it is possible to modify the width (the scale) and the position at which it is located. The correlation between a wavelet, with a certain width and location, and the signal is therefore given by equation 5.2.

Increasing the scale of a wavelet has a similar effect as reducing the frequency of a sine function. Consequently, in a complete WT, the low frequency components of the signal will be represented by the high scale wavelet coefficients, while the high frequency components will be represented by the low scale coefficients. This will be discussed in more detail in section 5.2.2.

\section{Mother Wavelets}

Wavelet transforms have another key advantage over FTs; there are many types of wavelets that can be used. Each has its advantages and disadvantages depending on the shape and frequency of the signal features. Furthermore, some wavelets are designed for a specific function, image compression for example. Table 5.1 contains a list of common wavelet classes and a plotted example of each [147]. Each of the wavelet classes have particular properties but there is one property that a function must have in order to reconstruct the signal from the wavelet coefficients, it must be orthonormal with translation and scaling. Mathematically, this means that:

$$
\int_{-\infty}^{\infty} \psi_{a, b}(x) \psi_{j, k}^{*}(x) d x=\delta_{a, j} \delta_{b, k},
$$

where $\delta$ is the Kronecker delta and $\psi^{*}(x)$ is the complex conjugate of $\psi(x)$. This principle also holds in FTs where the Fourier amplitudes can only be calculated at integer multiples of the smallest possible frequency. A set of orthonormal wavelets which covers a range of scales and positions are constructed from a single wavelet known as the Mother Wavelet. The problem of orthogonality is a significant concern in WTs and will be addressed in the next section. 


\begin{tabular}{|c|c|c|}
\hline Family & Description & Example \\
\hline Haar & $\begin{array}{l}\text { Discontinuous and resembles a step } \\
\text { function. Same as the first Daubechies } \\
\text { wavelet. }\end{array}$ & \\
\hline Daubechies & $\begin{array}{l}\text { Compactly supported orthonormal } \\
\text { wavelets. Designed for discrete wavelet } \\
\text { analysis. Total of } 10 \text { Daubechies } \\
\text { wavelets are supported in MATLAB }{ }^{\circledR} \text {. }\end{array}$ & \\
\hline Symlet & $\begin{array}{l}\text { Nearly symmetrical wavelet proposed } \\
\text { by Daubechies as a modification to the } \\
\text { original Daubechies wavelets. Has very } \\
\text { similar properties to the Daubechies } \\
\text { wavelets. }\end{array}$ & \\
\hline Coiflet & $\begin{array}{l}\text { The wavelet function has } 2 N \text { (where } \mathrm{N} \\
\text { is the wavelet number) moments equal } \\
\text { to zero and the scaling function has } \\
2 N-1 \text {. The two functions have a sup- } \\
\text { port length of } 6 N-1 \text {. }\end{array}$ & \\
\hline Biorthoganol & $\begin{array}{l}\text { Has the property of linear phase which } \\
\text { is needed for signal and image re- } \\
\text { construction. Two different mother } \\
\text { wavelets are used, one for decomposi- } \\
\text { tion and one for reconstruction, result- } \\
\text { ing in interesting properties. }\end{array}$ & \\
\hline Reverse Biorthoganol & $\begin{array}{l}\text { Same as the Biorthoganol wavelets } \\
\text { but applied in the reverse order, } \\
\text { i.e. the reconstruction wavelet in the } \\
\text { Biorthoganol case is used for decompo- } \\
\text { sition, and vice versa. }\end{array}$ & \\
\hline Meyer & $\begin{array}{l}\text { The Meyer wavelet is defined in the fre- } \\
\text { quency domain [145] and is symmetric. }\end{array}$ & $v^{\prime} \| / h_{r}$ \\
\hline
\end{tabular}

Table 5.1: A list of 7 wavelet families that are regularly used with the wavelet toolbox in MATLAB ${ }^{\circledR}$. Many of these are designed for discrete wavelet transforms using dyadic scaling. 


\subsubsection{Types of Wavelet Transforms}

There are two types of WTs that can be performed on a signal depending on what type of data analysis the user would like to perform. For a detailed visualisation of the frequency components at different locations on the signal, it is often useful to use a continuous WT (CWT). In a CWT, the wavelet coefficient is calculated at many possible scales and positions. This has the disadvantage that a modified signal cannot be reconstructed from the wavelet coefficients because only a subset of the wavelets used in the decomposition will be orthonormal. A discrete WT (DWT), on the other, hand only uses a subset of possible positions and scales which conserve the orthogonality of the wavelets. As a result, the wavelet coefficients can be filtered and a new signal can be reconstructed.

\section{Continuous Wavelet Transform}

In contrast with DWTs, CWTs are designed for detailed analysis of the frequency components at different locations of the signal. It is therefore convenient to ignore the orthogonality property of the wavelets so that we may obtain the wavelet coefficients to any desired resolution. Furthermore, there is a lot more freedom in the shape of the mother wavelet allowing the use of wavelets that are better suited to the signal but not orthogonal. However, CWTs have the weakness that a modified signal cannot be reconstructed from the coefficients.

Figure 5.2 shows an example of a Raman spectrum of CV which has been transformed with the non-orthogonal gaus8 wavelet (the 8th Gaussian wavelet) using the wavelet toolbox in MATLAB ${ }^{\circledR}$. The Raman spectrum is plotted on top of the 2D CWT plot, in which the x-scale is the Raman shift, and the $y$-scale is the scale of the wavelet (or the intensity in the case of the Raman spectrum). The colour of the $2 \mathrm{D}$ plot corresponds to the wavelet coefficient with dark blue representing a large coefficient and white representing a small coefficient. In other words, the dark blue regions are where there is a significant correlation between a wavelet at that scale and position with the Raman signal at that position. At extremely small scales, the wavelet coefficient are relatively small as there is very little noise. As the scale increases, we start to see large coefficients appear at the peak locations. Finally, at extremely large scales, the coefficients are large for most positions. This is due to the fluorescence background which is present at all positions. As a result, the wavelet coefficients at different scales are representing different characteristics of the signal: at high 


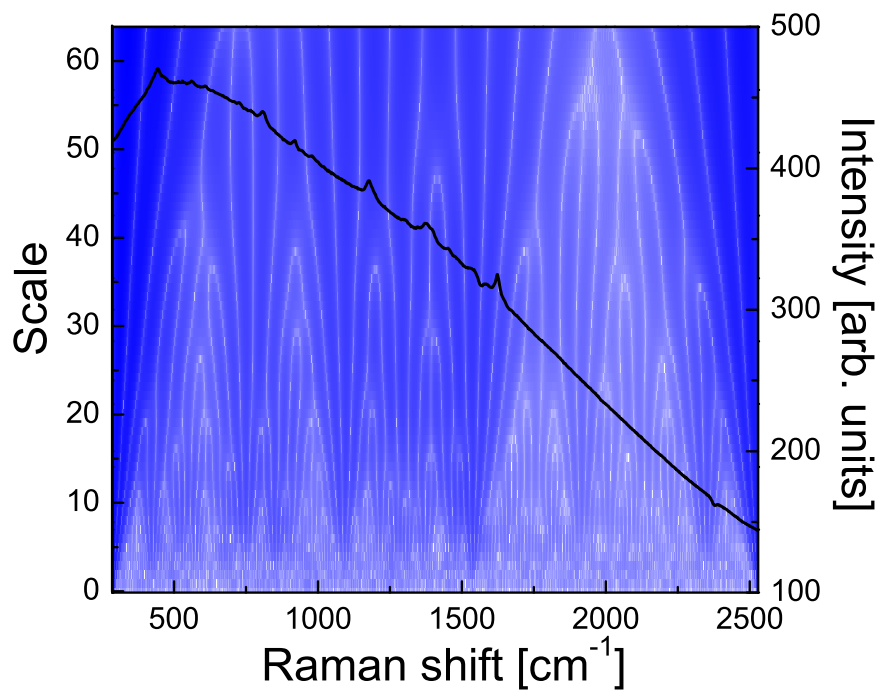

Figure 5.2: $A$

Raman spectrum of $C V$ along with a $2 D$ plot of the wavelet coefficients where the scale is plotted along the $y$ axis. Dark blue corresponds to a large wavelet coefficient while white corresponds to a small coefficient.

scales the coefficients represent the background contribution to the signal, at medium scales the coefficients represent the peak contribution to the signal, and at low scales the coefficients represent the noise contribution to the signal. One will also notice that coefficients close to the peaks (and the edge of the signal) have large coefficients as the scale increases. This is because part of the wavelet overlaps with the peak (or signal edge) at larger scales which causes the coefficients to increase.

\section{Discrete Wavelet Transform}

If we are free to continuously vary the scale and position, it is very difficult (if not impossible) to conserve orthogonality between wavelets. Instead, Daubechies [131] proposed the use of dyadic scaling and translation, such that the set of coefficients is constructed from wavelets that vary from each other like:

$$
\psi_{j, k}(x)=2^{-j / 2} \Psi\left(2^{-j} x-k\right), j, k \in \mathbb{Z},
$$

where $\Psi$ is the mother wavelet. Equation 5.5 states that for each increase in scale, both the width and shift in position of the wavelet is doubled. Using this principle, several sets of wavelets were designed that conserved orthogonality, some of which are shown in table 5.1.

Once the signal is transformed into discrete wavelet coefficients, defined by 


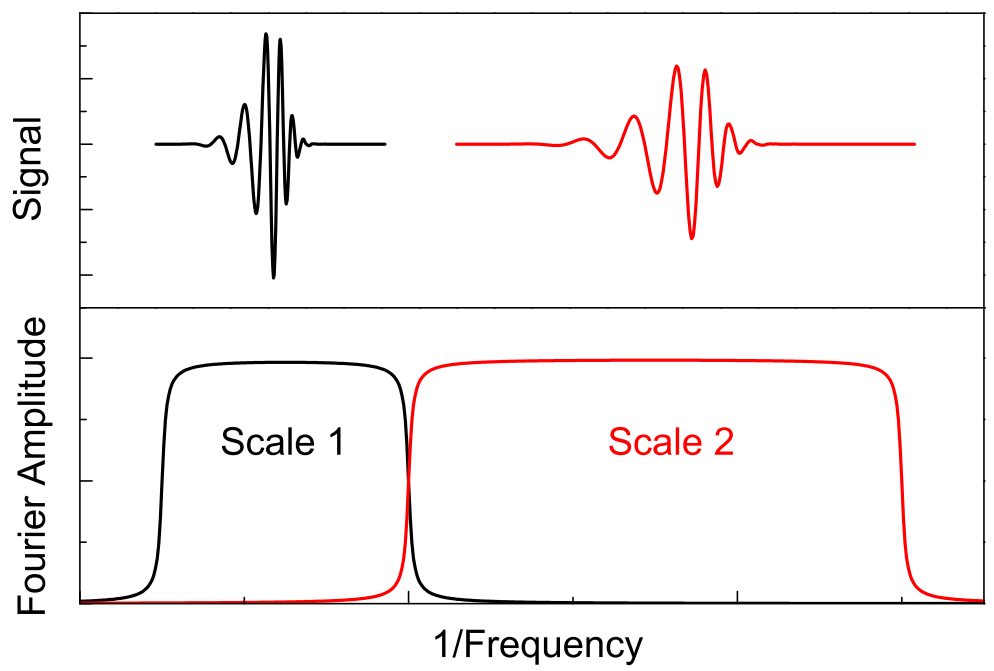

Figure 5.3: Two wavelets at scale 1 and 2 and their representation in the Fourier domain. Each acts as a band pass filter with the higher scale wavelet encompassing a lower frequency range. Furthermore, the frequency ranges of the wavelets do not overlap but, for any frequency, there will be a wavelet at a unique scale that covers it.

dyadic scaling and translation, filtering can be performed using thresholding (to be described in section 5.2.3). A filtered signal is then reconstructed from the thresholded coefficients. In the next section I will give a overview of a practical algorithm for DWT.

Many of the wavelets used for DWT also have the property that, in the Fourier domain, they have the same shape as a band pass filter and increasing the scale causes the frequency band to shift to lower frequencies as shown in figure 5.3. The full set of wavelets (with one mother wavelet) will encompass all possible frequencies. The advantages of using mother wavelets that have this property will become apparent in the next section.

\subsubsection{The Mallat Pyramidal Algorithm}

The Mallat Pyramidal Algorithm (MPA) was developed by Mallat in 1989 [132] as a multi-resolution signal decomposition technique for DWT. Using a combination of wavelet-based high and low pass filters, a signal is decomposed into subsets containing the different scale components of the original signal up to a defined level. In this section I will give a brief overview of the MPA without going into the detail of the mathematical formulation. Instead I direct the reader 


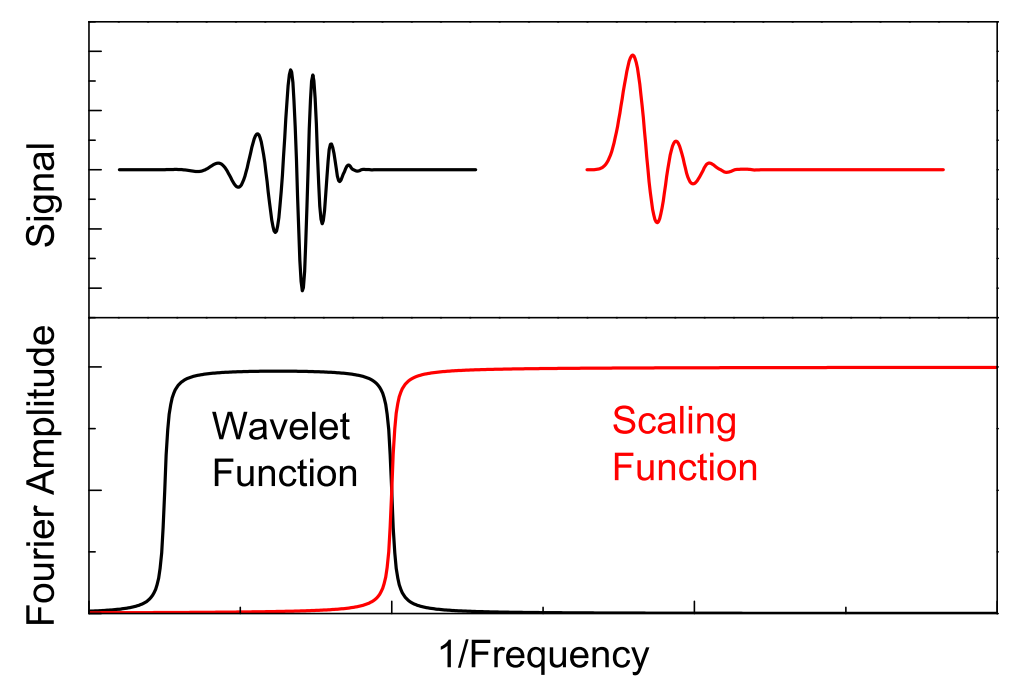

Figure 5.4: A wavelet and scaling function of the same scale and their representation in the Fourier domain. The wavelet function acts as a band-pass filter while the scaling function acts as a low pass filter. Together they cover all frequencies below the frequency band covered by the wavelet function.

to reference [132] if further details are required.

\section{Decomposition}

In section 5.2.1 I explained that in the frequency domain wavelets act as band pass filters. As a consequence, in order to encompass the whole frequency regime, we need an infinite number of wavelets. Mallat came up with a solution to this problem using quadrature mirror filters and scaling functions. For each wavelet used in the MPA, there is a mirror scaling function that acts as a low pass filter and encompasses all frequencies below the frequency band covered by the wavelet function (see figure 5.4). In reference [132], Mallat introduced the concept of scaling functions which are designed along with the wavelet function. Consequently, the scaling function is often referred to as the father wavelet.

Instead of decomposing the signal into an infinite number of wavelets, we use multiple resolutions of decomposition with wavelets and scaling functions. Let us first consider a signal with components at all scales for all positions. Using the lowest scale wavelet as a high pass filter and the mirror scaling function as a low pass filter, we can separate the signal into two parts (see figure 5.5); the discrete detail coefficients, $\mathrm{cD}$ (or just detail coefficients), which contain the high 
Figure 5.5: Diagram showing the first two levels of decomposition in the Mallat pyramidal algorithm. Each level of decomposition consists of separating the signal (or approximation coefficients) using a wavelet and scaling function based filter. The number of coefficients is also down-sampled after each stage of filtering to halve the number of coefficients without loss of information.

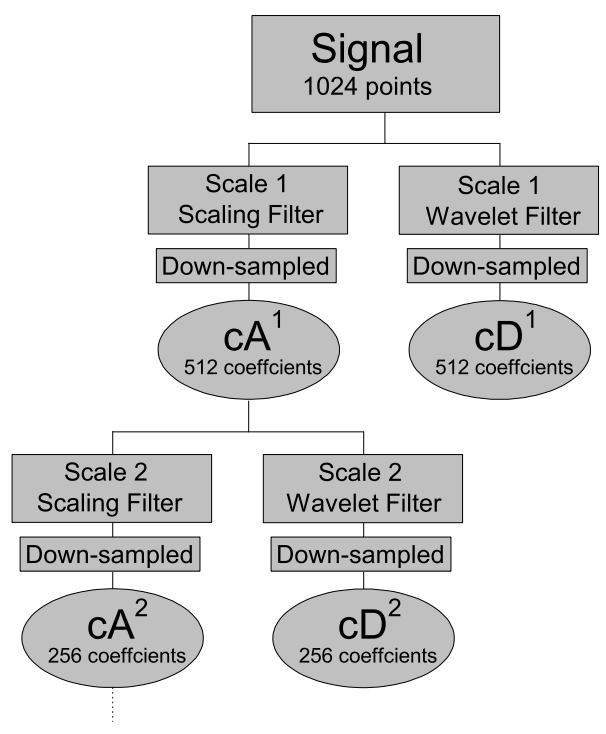

frequency information, and the discrete approximation coefficients, cA (or just approximation coefficients), which contain the low frequency information. The detail coefficients are the wavelet coefficients for the lowest scale wavelet and the approximation coefficients are the scaling coefficients for the lowest scale scaling function. Together the wavelet and scaling functions cover the full frequency domain and, hence, all information about the signal will be contained within the new set of coefficients.

The signal can be further decomposed by increasing the scale of the wavelet and scaling function, using dyadic scaling, and then repeating the process on the first set of approximation coefficients $\left(\mathrm{cA}^{1}\right)$. Each level of approximation coefficients, therefore, contains lower frequency information of the original signal while the higher frequency components are removed in the detail coefficients at different levels.

The wavelet and scaling coefficients also have the advantage that they can be down-sampled without any loss of information, such that there are half the number of wavelet (or scaling) coefficients as there are data points in the original signal or the input discrete coefficients. This can be particularly beneficial when decomposing the signal into many scale levels, as the total number of coefficients will be close to the number of data points, independent of the decomposition level. The decomposition can therefore be carried out until there is only a single coefficient left in the last discrete approximation. 
The decomposition of a RH6G SERS event using MPA is shown in figure 5.6 up to a decomposition level of 7 . The actual scaling and wavelet coefficients are not shown but have been reconstructed into detail and approximation spectra by taking the linear combination of the coefficients with the appropriate wavelet (or scaling) functions (discussed later in this section). After each level of decomposition, a frequency band is removed, defined by the wavelet function in the frequency domain, resulting in an approximation spectrum that consists of only the lowest frequency components of the signal.

\section{Thresholding}

Once the signal has been fully decomposed, we need a technique for modifying the wavelet and scaling coefficients before reconstructing a modified signal. The process of modifying coefficients is called thresholding and there are two main types of thresholding $[133,147]$ that are typically performed:

Hard thresholding All coefficients less than the threshold value are set to zero. All coefficients larger than the threshold remain the same.

Soft thresholding All coefficients less than the threshold value are set to zero. All coefficients larger than the threshold are reduced by the threshold value such that:

$$
W=\operatorname{sign}(W)(|W|-\lambda),
$$

where $\mathrm{W}$ is the coefficient and $\lambda$ is the threshold value.

Which type of thresholding is used depends on the type of signal modification that is required.

\section{Reconstruction}

Once the signal has been decomposed and modified with thresholding, we need a way to reconstruct it into a filtered signal. This is achieved by essentially reversing the decomposition process. The discrete detail and approximation coefficients (after thresholding) are first up-sampled and then passed through the inverse low and high pass filters to reconstruct the previous level of discrete approximation coefficients (see figure 5.7). This process is then repeated on the newly reconstructed approximation coefficients and the detail coefficients at the same level. Once all of the filtered coefficients have been reconstructed, we 


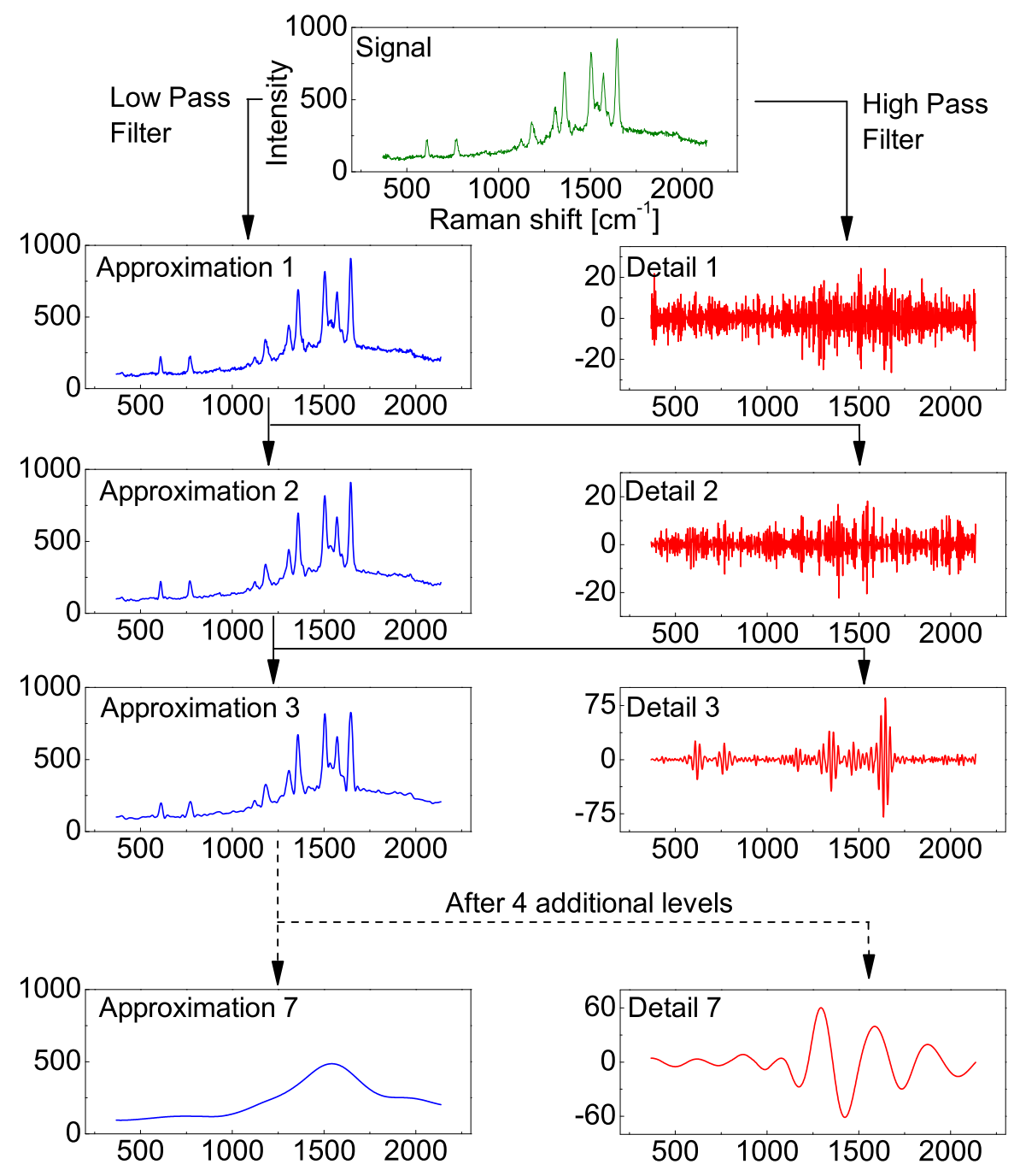

Figure 5.6: Diagram of the decomposition of a RH6G SERS signal down to 7 scale levels. Shown are the detail and approximation spectra instead of the actual coefficients. 


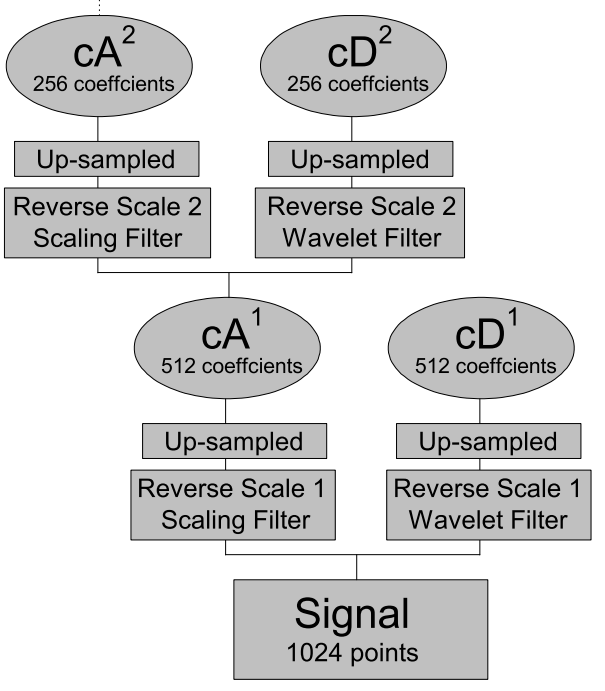

Figure 5.7: Diagram showing the first two levels of reconstruction in the Mallat pyramidal algorithm. Each level of reconstruction consists of up-sampling the detail and approximation coefficients and passing them through the appropriate reverse filters.

are left with a new modified signal. If no thresholding was performed, the new signal will be the same as the original one.

It is also possible to reconstruct approximation and detail spectra which are the real parts of the original signal (with the same number of data points) with frequency components defined by the scale of the wavelet. A detail spectrum at a certain scale is defined as the linear combination of all the detail coefficients at that scale, and the corresponding wavelets:

$$
D_{j}(t)=\sum_{k} c D_{j, k} \psi_{j, k}(t)
$$

where $c D_{j, k}$ is the detail (wavelet) coefficient with scale $\mathrm{j}$ and position $\mathrm{k}$. We can therefore write the original signal as a sum of detail spectra for all scales:

$$
S=\sum_{j, k} c D_{j, k} \psi_{j, k}(t)=\sum_{j} D_{j}(t)
$$

If we have detail spectra up to a certain scale $n$, and an approximation spectrum at scale $\mathrm{n}$, then:

$$
S=\sum_{j} D_{j}(t)=\sum_{j=1}^{n} D_{j}+\sum_{j=n+1}^{\infty} D_{j}=\sum_{j=1}^{n} D_{j}+A_{n}
$$

As a result, the sum of all of the detail spectra and the final approximation spectrum will be the same as the original signal if no thresholding is performed. 

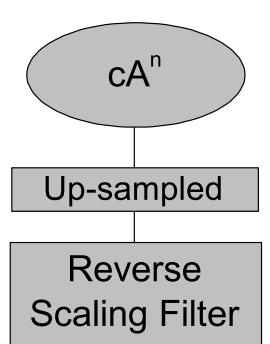

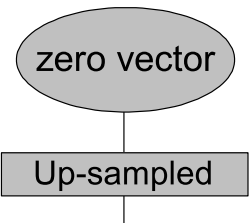

Reverse Wavelet Filter

Approximation Spectrum 1024 points

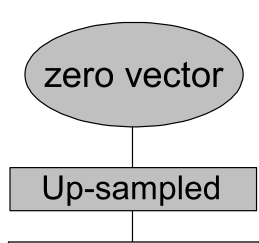

\section{Reverse Scaling Filter}

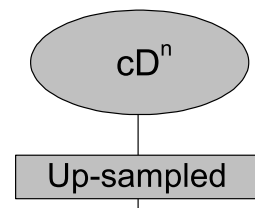

Reverse

Wavelet Filter

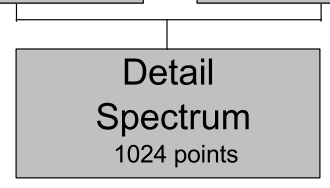

Figure 5.8: Diagram of the reconstruction of an approximation and detail spectrum of scale $n$. The approximation spectrum consists of filtering the approximation coefficients and a zero vector, while the detail spectrum consists of filtering the detail coefficients and a zero vector.

To create the approximation and detail spectra, we combine the coefficients with a zero vector as shown in figure 5.8. Once the coefficients are converted into detail and approximation spectra, the original signal can be reconstructed by taking the sum of any approximation spectrum and all detail spectra at and below the scale level of the approximation.

\subsection{Wavelet Transforms in Signal Processing}

There are many applications in signal processing that have utilised WTs including noise reduction [143,149-154], spike removal [151], and background subtraction $[142,143,154-157]$. All of these techniques are dependent on being able to separate a signal into frequency domains which have different physical origins. In this section I will give an overview of the three main frequency domains that distinguish the main signal features, at least in the case of Raman spectroscopy. I will also discuss the basic ways a signal can be modified using WTs.

\subsubsection{The Three Frequency Regimes}

It is not uncommon in signal processing to be working with spectra that contain three main characteristic sources, having particular frequency regimes and representing different features of the data. An example of a SERS spectrum is 
shown in figure 5.9, highlighting the contributions that are distinguishable in the frequency domain.

High frequency noise Noise is present in practically all forms of signal processing and there are many techniques for dealing with it. FTs are generally very good in this case, because noise is typically dominated by high frequency components and, hence, the finite window size is not a problem [158]. In DWTs most of the noise will be contained within the first few sets of detail coefficients.

Medium frequency signal peaks The most interesting part of the signal is typically in the form of peaks. For example, the Raman peaks in a SERS signal. These features are generally in the medium frequency range and, unlike noise which is present throughout the spectrum, signal peaks are most often "localised" at specific positions (Raman shifts in our case). It is because of this localisation that WTs are a much better option for analysing this component of the data compared with FTs which require a large number of sine functions to represent a single peak.

Low frequency backgrounds Backgrounds are present in many different types of signals and due to their "low frequency" nature, are very difficult to treat. Furthermore, unlike noise, the shape of the background can have a physical meaning. In SERS of resonant or pre-resonant molecules, for example, the background is primarily produced by SEF, and information about the fluorescence spectrum (and the underlying plasmon resonances that affect it) can be drawn from its shape and intensity [73]. However, FTs have a lot of difficulty dealing with these backgrounds as the frequency components can have periods that are similar to or larger than the spectral window. This is a case where the real strength of WTs as a processing tool is clear compared with the more conventional option of FTs.

When performing a DWT, different frequency regimes will be removed in the detail coefficients at each level. For a typical 1024 point Raman spectrum (a typical number of data points for spectra obtained with CCD-detectors), most of the noise will be removed in the first two levels, while most of the Raman peaks will be removed in the $3^{\text {rd }}$ to $5^{\text {th }}$ levels. At the $6^{\text {th }}$ or $7^{\text {th }}$ level of decomposition, the background will be the dominant contribution to the approximation spectrum. However, this is not strictly the case, especially when 


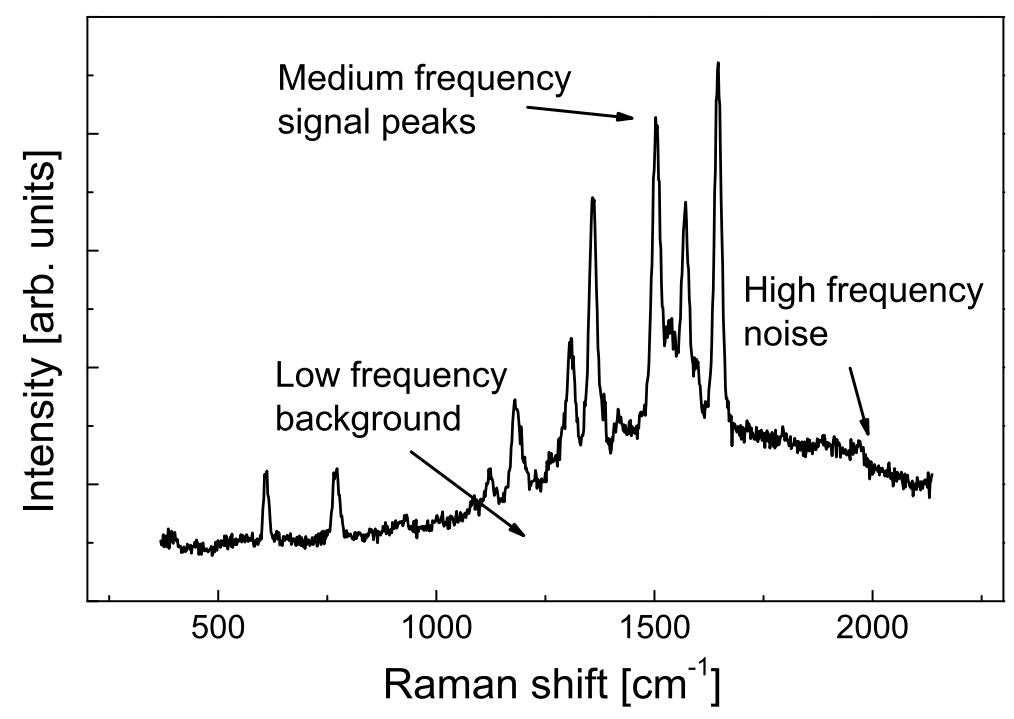

Figure 5.9: A SERS spectrum of RH6G demonstrating the three different contributions to the overall signal; high frequency noise, medium frequency Raman peaks and the low frequency background.

the Raman peaks are close together. This problem will be addressed later in the chapter.

\subsubsection{Signal Modification using Wavelet Transforms}

Let us consider an ideal case in which a signal is constructed by the three contributions listed above, each of which are contained within a region in the frequency domain and do not overlap (see figure 5.10). Using MPA, the signal can be decomposed such that each of the signal contributions will be contained within different sets of detail coefficients. Detail levels $1-3$ will encompass all of the high frequency noise whilst the $4^{\text {th }}-7^{\text {th }}$ levels will comprise of the signal peak components. The background components will therefore be completely contained in the $7^{\text {th }}$ level approximation coefficients.

The noise can be completely removed by simply setting all of the detail coefficients in the first three levels to zero and then reconstructing the signal as explained in section 5.2.3. Furthermore, the low frequency background can be removed by constructing the $7^{\text {th }}$ level approximation spectrum and subtracting it from the reconstructed signal. The remaining signal will therefore only consist of the signal peaks. 


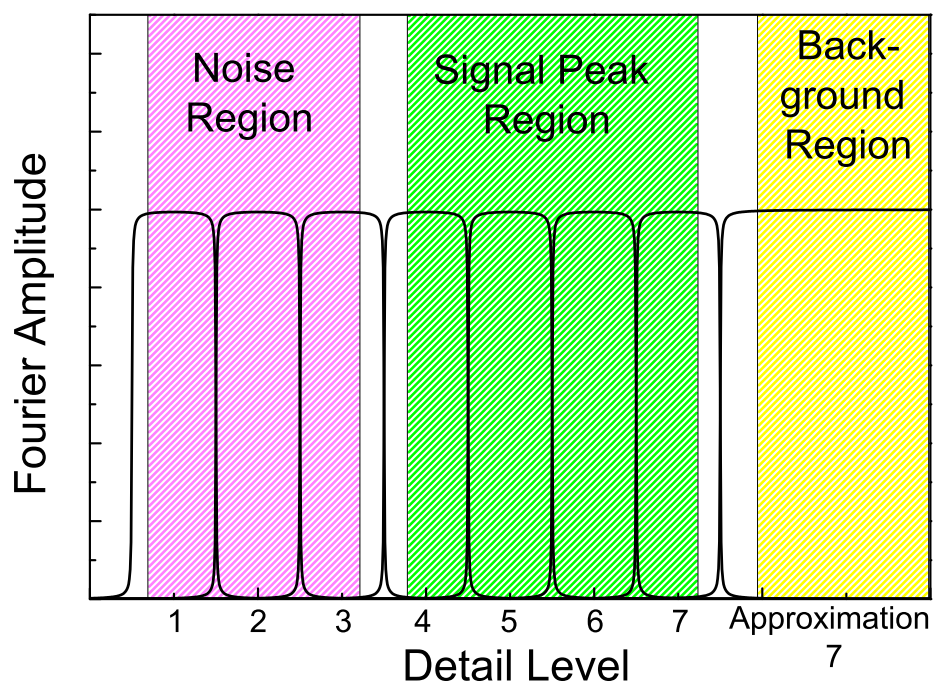

Figure 5.10: The frequency bands of the three different signal contributions when there is no overlap between them. Also shown is the frequency region for each level of detail. The noise is contained within the first three details levels, the signal peaks within the $4^{\text {th }}-7^{\text {th }}$ levels, leaving only the background in the $7^{\text {th }}$ approximation. This is adapted from figure 1 in reference [2].

However, most physical cases will have overlapping contributions, particularly the overlapping of the signal peaks and the noise or background. In the case of overlapping peaks and noise (in the frequency domain) it is common to use thresholding to filter the low scale detail coefficients instead of setting them all to zero. Because the signal peaks are typically larger than the amplitude of the noise, the coefficients corresponding to the peaks will typically be larger than the coefficients that correspond to noise. Figure 5.11 shows an example of a SERS spectrum of CV in which the first two detail level coefficients, which are completely dominated by the noise contribution, are set to zero but the third level coefficients, which have a contribution from the noise and Raman peaks, are filtered differently. In figure 5.11(a) there is no filtering of the coefficients and, as a result, there is a significant level of noise relative to the other two situations. Figure 5.11(b) shows hard thresholding of the coefficients such that the threshold value is above the noise coefficients but below the peak coefficients. There is significantly less noise in this case while leaving the peaks unmodified. However, in figure 5.11(c), where all of the coefficients have been set to zero, the peak resolution is significantly reduced and the peak intensities are slightly lower than in the previous two situations. As a result, hard thresholding with a 
Figure 5.11: A modified SERS specrtum of $C V$ in which the first two levels of detail coefficients have been set to zero, but the third level coefficients are (a) unmodified, (b) hard thresholded, and (c) set to zero. Each spectrum also contains an inset plot showing the third level detail coefficients with the appropriate thresholding.

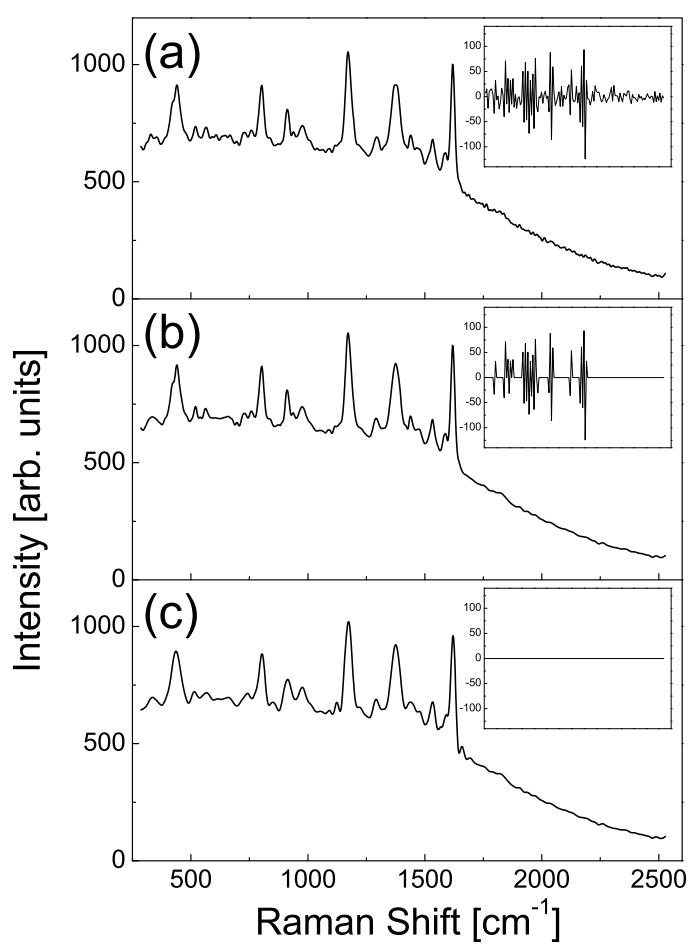

suitable threshold value gives the best results for de-noising without modifying the peak shapes.

An overlap of the peak and background contributions is much more difficult to treat as many of the coefficients at high detail levels will be affected by both contributions. Any modification of these coefficients could therefore alter the peaks and background significantly. Furthermore, assuming that the high level approximation spectrum is a good representation of the background will result in a background fit that is significantly modified by the presence of the peaks. There are many examples in signal processing, including SERS, that suffer from this short-coming. This is one of the problems that has lead us to develop an algorithm based on WTs for the removal of backgrounds from large numbers of SERS spectra. 


\subsection{The Iterative Algorithm for Background Re- moval and the COBRA}

In the previous section I described the types of signals that are often observed in spectroscopy and SERS in particular. In the case of SERS, the background signal is dominated by the SEF spectrum of the molecules while the signal peaks are created from their SERS spectrum. Both are enhanced by the plasmon resonance but not in the same way [76] (see section 3.2). As a result, being able to separate each contribution to the signal is very desirable. There are, however, three properties that make the separation of the background very difficult for these types of signals.

Firstly, dealing with large numbers of spectra requires an algorithm that minimises user input. For example, an algorithm that requires the user to define the threshold value for every spectrum would not be practical. For a few tens of spectra, the backgrounds can be subtracted by hand or using a commercially available background removal application (such as that available in Origin $\left.{ }^{\mathrm{TM}}[159]\right)$. However, a different method is required when treating thousands (or tens of thousands) of spectra, as is often the case when performing time series or spatial mappings in SERS.

Secondly, the algorithm must be able to reliably and efficiently perform the background subtraction on signals with extremely variable backgrounds. Furthermore, it must not introduce artifacts in the process. If the background is very similar between spectra, a relatively easy background subtraction for all spectra could be achieved. However, in areas such as single molecule SERS the background (and signal peaks) can vary significantly in both shape and intensity between events (due to the sensitivity of the signal to the local environment of the molecule). As a result, the frequency region of the background will be different for each event, in some cases there may even be an overlap between the two regions.

This brings us to the final property, the algorithm must be able to address the overlap of the frequency regions of the background and peaks. The frequency region of the noise and peak contributions are reasonably predictable in the case of SERS, as the peak width is not affected by the dispersion in the plasmon resonance. The relative intensities of the peaks will change due to the plasmon resonance favouring scattering of certain Raman modes, but this will only modify the amplitude of the wavelet coefficients, not the scale. The SEF 


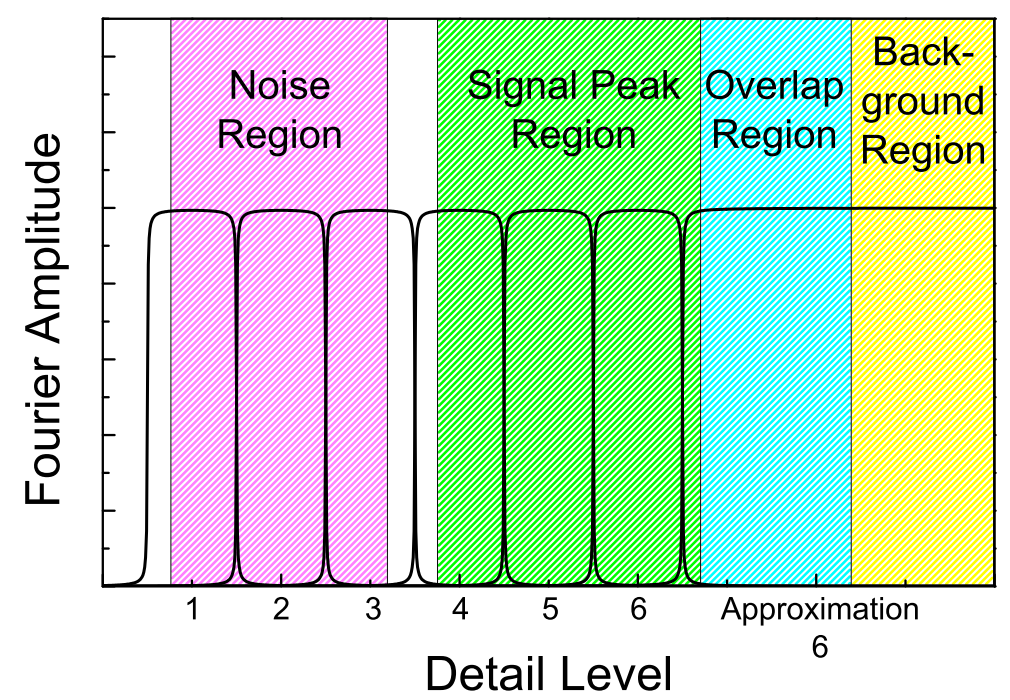

Figure 5.12: The noise, signal peak and background regions in the frequency domain for a high frequency background signal event. The background is completely contained within the $6^{\text {th }}$ level approximation coefficients, however, there is an overlap with the signal peak region which also extends into the $6^{\text {th }}$ level approximation coefficients.

spectrum on the other hand is continuous and the frequency is modified by the properties of the plasmon resonance. As a result, the frequency region of the background will often overlap with the frequency region of the peaks. In this situation, the background cannot be separated from the rest of the signal by a direct modification of the detail or approximation coefficients (as was discussed in section 5.3.2).

Any algorithm that will be suitable for our purposes must therefore be able to address these problems.

\subsubsection{The Iterative Algorithm}

There is a key advantage to using WTs to filter the signal: if the background can be removed for the highest frequency background signal, then it should be easily extendable to all events. The reason for this is described below.

Let us take the case of a set of spectra in which the frequency region of the noise and peaks are fixed but the background region can vary freely. The background and noise regions may also overlap with the peak region. This is a good representation of a SERS mapping in which a typical SERS analyte (RH6G for example) is being probed. Taking the spectrum with the background that has 

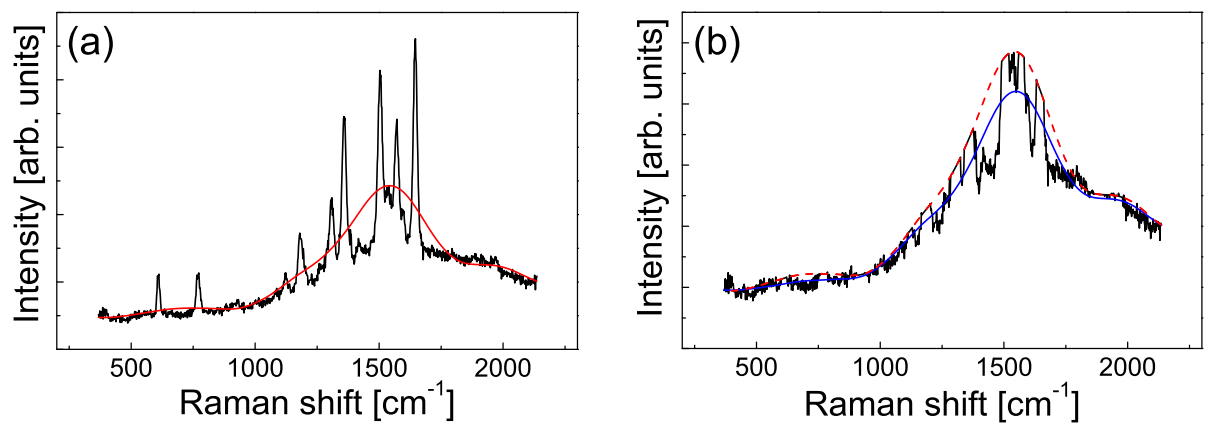

Figure 5.13: A RH6G SERS spectrum undergoing one iteration of modification using the $10^{\text {th }}$ Daubechies wavelet. (a) shows the original signal (black line) along with the $7^{\text {th }}$ level approximation spectrum (red line). (b) shows the signal after it has been modified (black line) as well as the original approximation spectrum (red dashed line) and the new approximation spectrum (blue line) resulting from the decomposition of the modified signal. This figure is adapted from figure 2 in reference [2].

the highest frequency coefficients, we decompose it so that all of the background and some of the peak contribution is contained within the approximation coefficients (see figure 5.12). The approximation spectrum is then constructed as described in section 5.2.3.

The next step is to remove the contribution of the signal peaks from the approximation spectrum. This is achieved by comparing the original signal to the reconstructed approximation spectrum and modifying the signal so that all points larger than the approximation spectrum are set equal to the approximation spectrum (see figure 5.13). We are then left with a new modified signal which still has signal peaks but they are significantly smaller than the signal peaks in the original signal.

Often there will be situations where the fit will be below the signal in regions that are purely background. Typically this will occur close to the boundaries, but there may be additional regions, near a large group of closely packed peaks for instance. In these situations it is useful to define regions that the algorithm can assume are purely background and will never be modified. How critical the background regions are will be investigated later in the chapter.

The decomposition is then repeated on the modified signal to the same scale and a new modified signal is created using the identical process. This is repeated until the final approximation spectrum converges (i.e. does not change after an additional signal modification). Each iteration of this process removes part of 


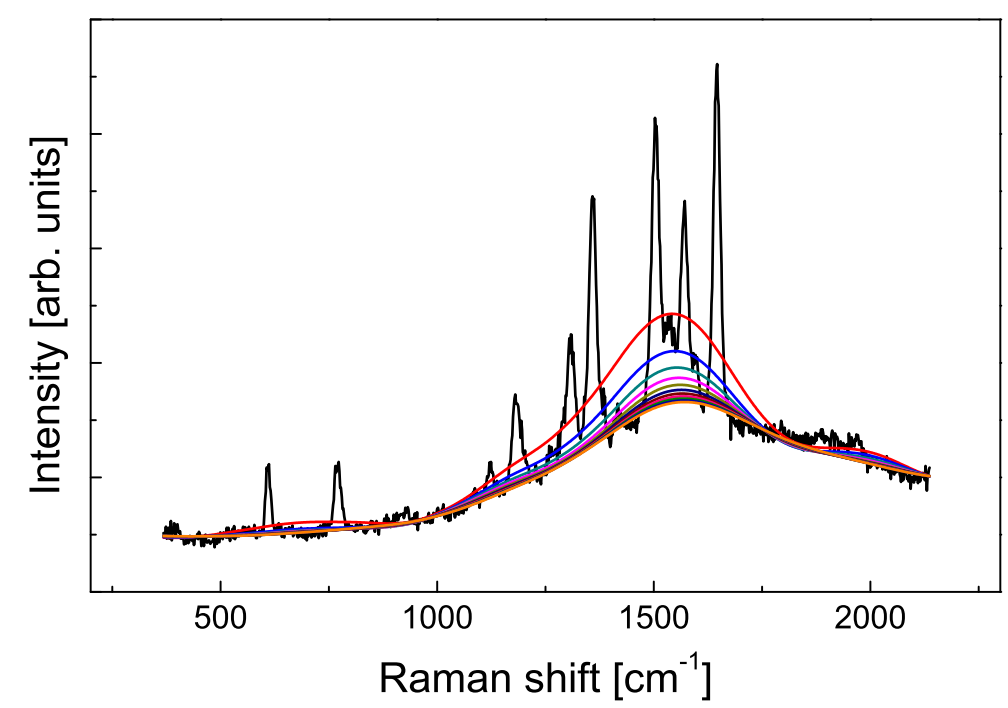

Figure 5.14: The convergence of the background fit for the RH6G spectrum shown in figure 5.13. Shown is the th level approximation spectrum after each modification of the signal. After 10 iterations, the approximation spectrum has converged on the background. This figure is adapted from figure 2 in reference [2].

the signal peaks from the signal until it finally converges on the background. An example spectrum of RH6G is shown in figure 5.14 along with the approximation spectrum after each iteration. By the $10^{\text {th }}$ iteration the approximation spectrum has converged on the background. It is this final approximation that we call the background fit. Subtracting the background fit from the original signal will therefore result in a background removed signal containing only noise and peaks.

Decomposing all of the signal spectra to the same detail level as we have for this case and repeating the iterative process of signal modification, will then result in a complete set of background removed spectra.

In order for this algorithm to be suitable for our purposes it must address the problems listed in the previous section. Firstly, can it deal with large numbers of spectra with very little user input? There are several parameters that need to be set for the background removal of all spectra:

Wavelet Type A mother wavelet that is best suited for the signal shape. This will depend on the shape of the signal peaks which will be similar for all signal events.

Decomposition Level Each spectrum needs to be decomposed to a level such that the background contribution is completely contained within the final 
set of approximation coefficients. By determining the appropriate level from a high frequency background event, decomposing all spectra to that same level should ensure that this is always the case.

Iteration Number The number of signal modifications that need to be performed in order for the background fit to converge. Typically the largest signal events will require the most iterations for convergence.

All of these parameters can be determined by performing the background removal on one or two events and then using the same parameters on all other spectra. Typically, taking the largest signal event and determining all three parameters is sufficient for the complete background removal of all events. However, there may be situations where the background frequency of the largest event is relatively low and a lower decomposition level will need to be used for some spectra.

The second problem of the signals with variable backgrounds is addressed by the decomposition level. As long as the parameters are set so that the highest frequency backgrounds can be removed, the background from all the other signal events can be removed using the same decomposition level, independent of how they vary relative to each other. Artifacts may however be introduced if the overlap regions become too large. For example, when the peaks are very closely packed and the background frequency is very high. The approximation coefficients will contain such a large contribution from the signal peaks that they may never be completely removed regardless of how many iterations are performed. Defining additional background regions between signal peaks will occasionally solve this problem (as I will show in an example later in this section).

Finally, the overlap of background and peak contributions in the frequency domain. The iterative algorithm addresses this problem by reducing the size of the signal peaks after each iteration. Eventually the peaks will be completely removed from the signal leaving only the background and noise.

\subsubsection{The COBRA}

For the complete de-noising and background removal of a single spectrum (or a set of spectra) we use several additional procedures, most of which are optional and situational. The accumulation of these procedures is brought together in a MATLAB ${ }^{\circledR}$-based background removal application, also called the COBRA, which was developed during this thesis. 


\section{The Bare Background Spectrum}

It is often useful to remove a bare background spectrum from the signal before performing the background fit. For example, in SERS there is often a contribution to the signal from water and impurities. It is therefore advantageous to subtract a spectrum of the solution without the metallic nano-particles from each of the signal events. The remaining background will then be due to fluorescence of the SERS molecules which may need to be accurately measured (as will be the case in the next chapter). This is however quite specific to SERS; it might not be necessary or even relevant in other situations.

\section{Polynomial Fit}

An initial low order polynomial subtraction can be performed as a pre-conditioning on the data before the iterative algorithm is performed. The objective of this step is not to accurately fit the background but to remove overall "upward" or "downward" trends in the background, which WTs may find difficult to deal with. The polynomial fit will also be performed iteratively as with the wavelet background fit (discussed earlier). The order of the polynomial and the number of iterations must therefore be defined in this stage.

\section{Background Regions}

As mentioned earlier, regions that are purely background can be defined in order to help with the iterative wavelet-based background fit in the next step. A region at each end of the signal will typically need to be defined independent on the shape of the signal. This is because the WT will struggle to converge on the signal within one wavelet width of the edge. It will therefore become more important to define this region at larger decomposition levels.

There may also be cases where additional background regions need to be defined. Typically these will become more important for signals that contain overlapping peaks as in the case of $\mathrm{RH} 6 \mathrm{G}$ which has a dense region of high energy modes.

\section{Background Removal}

Once the signal has been adjusted for the bare background and polynomial fit (both optional), and the background regions are defined, the iterative algorithm explained in section 5.4.1 is performed. The parameters defined in this stage 
are the same as those explained earlier; the wavelet type, decomposition level and number of iterations. This is the only step in the application that is not optional and for some cases the background removal can be accurately achieved without prior modification of the signal and without defining any background regions.

\section{De-noising}

WTs and FTs are both very powerful techniques for de-noising a signal. However, we chose to use FTs and a low pass filter for our de-noising stage in the application for several reasons:

- FTs are better representations of non-localised, high frequency signal features like noise whereas WTs are a better representation of localised signal features like the peaks.

- For WTs, thresholding needs to be used to get the best de-noising results (as was shown in section 5.3.2) as there will typically be a scale level that encompasses both noise and signal peak contributions. However, the best choice of threshold value is very case dependent and will not be the same for all signal events. This is not the case for FTs where using a low pass filter with a cut-off at the transition from the noise to signal peak frequency region (see figure 5.15) will give the best results and be approximately the same for all events.

- A fast FT is typically faster than a DWT decomposition using the MPA.

The de-noising is performed after the background has been removed to simplify the FT and make the choice of filter parameters easier. The parameters that need to be defined are the cut-off frequency and width (which is the size of the frequency region that the filter changes between 0 and 1). In this way, DWTs and FTs complement each other and are used for two completely different purposes in problems where they can show their distinct (and different) strengths.

\section{Filter All Signal Events}

Each of the steps listed above can be performed on any of the spectra, but the spectrum with the largest overall signal is used as a default. Once the parameters have been defined for the chosen spectrum, they are applied to all 


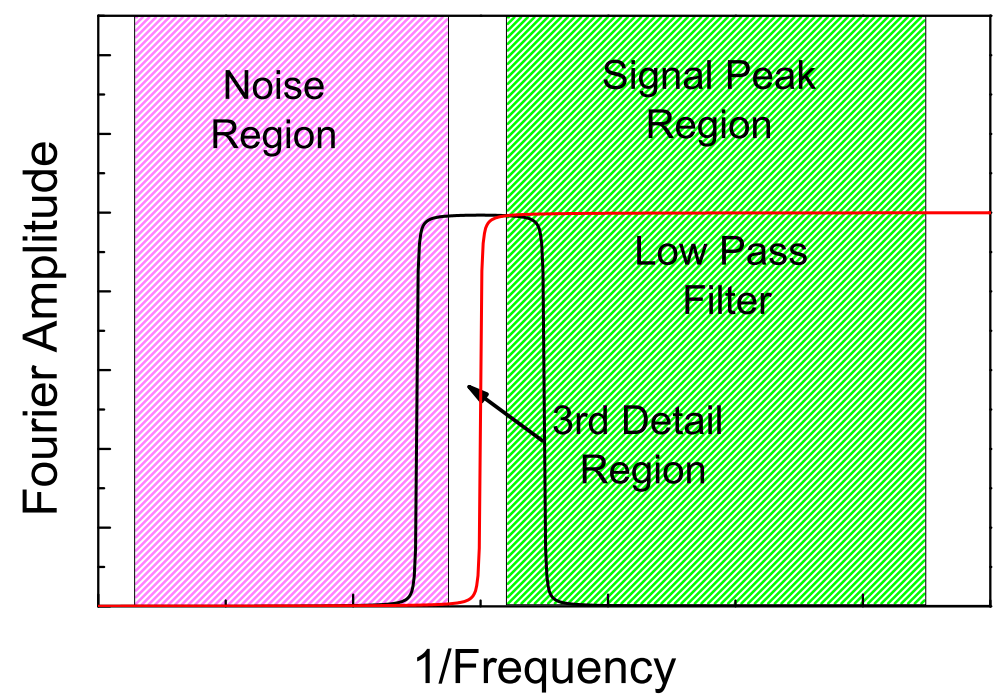

Figure 5.15: The noise and signal peak regions in the frequency domain. The third detail consists of a contribution from both signal peaks and noise and, as a result, thresholding is needed to properly de-noise the signal. The low pass filter on the other hand can be tailored such that all of the noise is cut out and only the signal peaks remain.

other spectra resulting in a complete set of background removed and de-noised spectra. At the end of this process, each of the spectra will be separated into background and filtered signals (the part of the signal above the background). The process of analysis (either linear decomposition of the Raman peaks in terms of reference spectra, or analysis of the background itself) can continue from there.

\subsubsection{A Simulated Signal}

In order to ascertain the accuracy of the background fit made with the COBRA, we need a signal in which we know the real background. This can be achieved by simulating a spectrum which consists of 1024 points (the same as a typical CCD readout) and has a background defined by a third order polynomial, peaks that have a Gaussian lineshape, and random noise. Figure 5.16 shows the simulated signal and the real background.

To test the accuracy of the iterative algorithm and the effect of defining background regions, the background fit is performed in six different ways. In each case we have chosen to use the Daubechies 10 wavelet and the iterative 


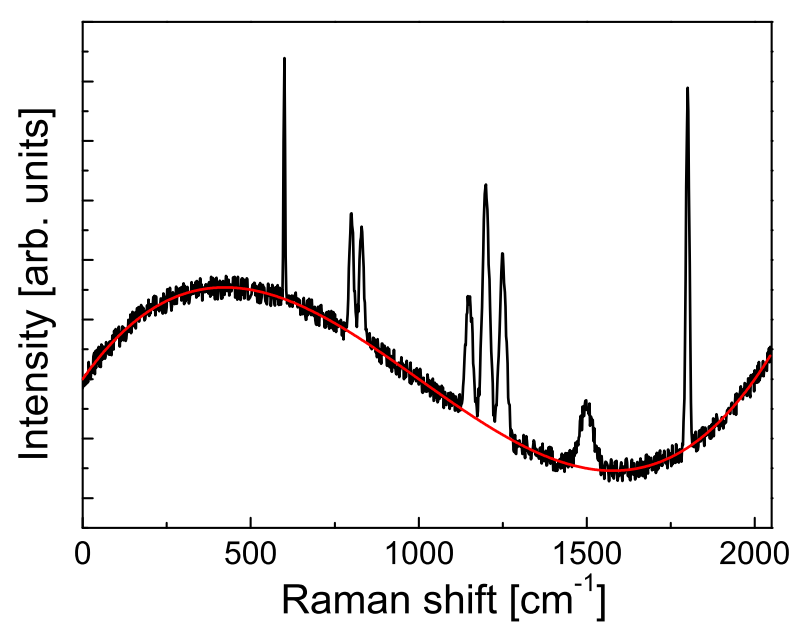

Figure 5.16: A simulated spectrum of 1024 data points, consisting of a third order polynomial background, Gaussian shaped signal peaks, and random noise. To test the capabilities of the COBRA we have included a relatively wide peak close to $\sim 1500 \mathrm{~cm}^{-1}$ and a collection of three closely packed peaks around $\sim 1200 \mathrm{~cm}^{-1}$. This figure is adapted from figure 3 in reference [2].

algorithm is performed for 10 iterations. This is typically enough to enable convergence of the background fit for most cases. Figure 5.17 shows the six different background fits, along with Table 5.2 which contains the parameters and the $\chi$ value which is defined as:

$$
\chi=\sqrt{\sum_{n=1}^{N}\left(y_{i}-f_{i}\right)^{2}},
$$

where $y_{i}-f_{i}$ is the difference between the plot and the fit at each point, and $\mathrm{N}$ is the total number of data points in each curve. The $\chi$ value is a measure of the accuracy of the fit.

Figures 5.17(a)-(d) show fits using the iterative algorithm, while figures 5.17 (e)-(f) have fits that use the approximation spectrum before any signal modification is performed. The best fit occurs using the iterative algorithm with a decomposition level of 6 , but with all of the background regions defined (see figure 5.17(b)). This is not surprising as only the peaks will be modified after each iteration. Often it is not possible, however, to define this many background regions. If we restrict ourselves to only defining the background at the boundaries, but keep the decomposition level the same (see figure 5.17(a)), the fit is greatly distorted close to the peaks; in particular the group of three peaks 


\begin{tabular}{|c|ccc|c|}
\hline Plot & \multicolumn{3}{|c|}{ Fit Parameters } & $\chi$ \\
& scale level & iterations & background regions & \\
\hline a & 6 & 10 & 2 & 435 \\
b & 6 & 10 & all & 120 \\
c & 7 & 10 & 2 & 426 \\
d & 7 & 10 & 3 & 279 \\
e & 7 & 0 & NB & 1111 \\
f & 8 & 0 & NB & 980 \\
\hline
\end{tabular}

Table 5.2: The fitting parameters used for the background fits in figure 5.17, as well as the $\chi$ values for the difference between the real background and the fit in each case.

at $\sim 1200 \mathrm{~cm}^{-1}$ and the wider peak at $\sim 1500 \mathrm{~cm}^{-1}$. Increasing the decomposition level to 7 (see figure 5.17(c)) significantly reduces the distortion, but there is still a region close to $\sim 1500 \mathrm{~cm}^{-1}$ where the fit drops below the signal. Defining a third background region on the right hand side of the $\sim 1500 \mathrm{~cm}^{-1}$ peak corrects this (see figure 5.17(d)). If we compare these fits with what is expected without the iterative process of signal modification (see figure 5.17(e) and(f)), we can see that the iterative algorithm outperforms the conventional choices. In particular, by comparing the fits in figures $5.17(\mathrm{c})$ and (e), we can see the advantage of the iterative algorithm. Both fits have a decomposition level of 7 in the DWT, but the former case goes through 10 iterations of signal modification while there is none in the latter. It is obvious in the second case that the signal peaks have a significant contribution to the fit. Increasing the decomposition level to 8 resulted in a significantly better fit for the non-iterated process (see figure $5.17(\mathrm{f})$ ) but this was not as good as with the iterative algorithm.

By looking at the $\chi$ values in Table 5.2 for plots $a$ and $c$, one might assume that the accuracy of the fit is independent of the decomposition level. But if we look at the fits in figures 5.17(a) and (c) it is obvious that the latter is a much better representation of the background. In the former case, the fit is very close to the real background but with slight distortions close to the peaks which will lead to incorrect estimates of the peak properties (the peak intensity, for example). The latter case, however, follows the actual background quite closely but with a significant distortion close to the $\sim 1500 \mathrm{~cm}^{-1}$ peak. This was corrected by defining a third background region to help in the convergence of the algorithm. 


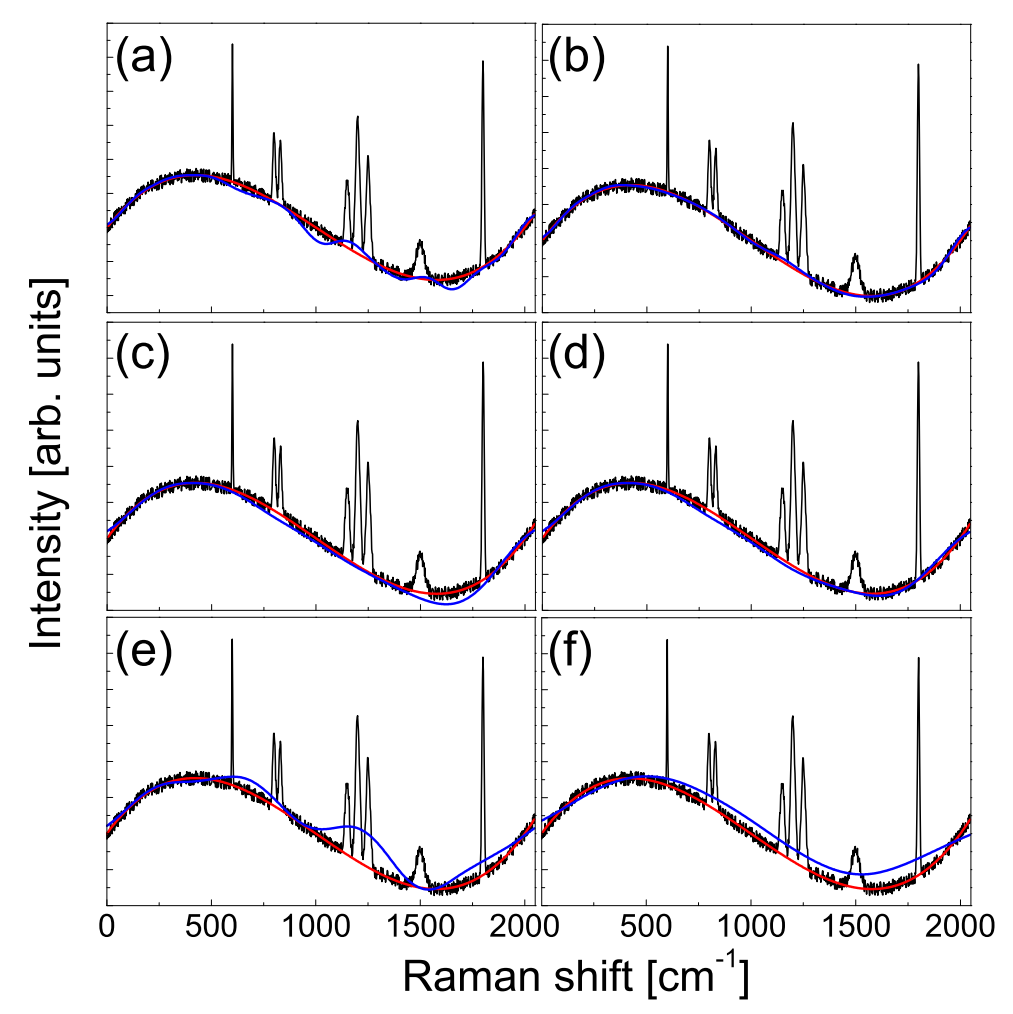

Figure 5.17: The simulated spectrum shown in figure 5.16 with six different types of wavelet based background fits with the fitting parameters defined in table 5.2. (a)-(d) are fitted using the iterative algorithm while (e) and (f) are fitted with the non-iterative approximation curve. In all cases the red curve represents the "real" background, while the blue lines are the fitted versions. See table 5.2 for complementary information on the different fits. This figure is adapted from figure 4 in reference [2]. 


\begin{tabular}{|l|l|l|}
\hline Fit Type & Parameter Name & Typical Value \\
\hline \multirow{2}{*}{ Polynomial } & Polynomial Order & 1 \\
& Iteration Number & 5 \\
\hline \multirow{3}{*}{ Background } & Wavelet Type & Daubechies 10 \\
& Decomposition level & $6-7$ \\
& Iteration Number & 10 \\
\hline
\end{tabular}

Table 5.3: Typical parameters for the polynomial and background fit (using the wavelet-based iterative algorithm)

\subsubsection{SERS Applications for the COBRA}

It remains now to show how the procedure described hitherto applies to real (SERS) data. We shall now present two different experimental results that are completely independent of each other but both of which require the separation of the SEF background from the SERS peaks. The parameters used for the polynomial and background fits (using the iterative algorithm) for a typical SERS spectrum taken with our experimental setup are given in table 5.3. There will however be slight differences depending on the case.

\section{Example 1: Background of Varying Intensities}

The first example looks at how the SERS peaks and underlying SEF intensities change as the SERS probe is moved closer to or further away from a SERSactive substrate by means of an electric field [160]. This example is relevant for the present thesis in the sense that it shows how background and signal can be decoupled, a fact that we are going to use in the single molecule studies. In this case, the background is relatively broad and its shape remains similar from one spectrum to the other; only its intensity (relative to the peaks) vary. What makes the separation of Raman peaks from the fluorescence important in this experiment is their different origins. The full details of this study and its physical interpretation can be found in reference [160]. We only focus here on the background removal process.

Using the COBRA it was possible to obtain an accurate fit to the background and subtract it from the original spectra as shown in figure 5.18. This was carried out automatically (without user intervention) on a large number of spectra (721 spectra as a function of time). The frequency region of the background signal is extremely similar between events and hence any spectrum could be chosen to define the fitting parameters. The background fit was per- 
formed to the $6^{\text {th }}$ decomposition level as the SERS peaks are relatively isolated. Furthermore, a background region at the boundaries of the signal was defined. Even though we could define additional background regions between each of the Raman modes, this was all that was needed to obtain an accurate background fit for all spectra. This was because there was very little overlap between the peak and background regions in the frequency domain.

In figure 5.18, the spectra with the largest and smallest overall intensity are chosen (along with the first measured spectrum). These two cases show the largest difference in fluorescence shapes and yet the background fit is extremely accurate for both. However, this example is not particularly challenging because the background is fairly predictable from one spectrum to the next and many other background removal tools could probably be used and obtain the same results.

Also provided in 5.18 are the background fits that would be predicted if there was no signal modification and only the first approximation 7 spectrum had been used. As we can see, the iterative algorithm is a much better approximation of the real background. In the non-iterative case, the $7^{\text {th }}$ approximation spectrum was plotted instead of the $6^{\text {th }}$ because the peaks greatly distort the fit in the latter case. This does not happen for the iterative plot in which the ability of the peaks to distort the fit is reduced during the iterative process.

\section{Example 2: Single-Molecule SERS Spectra}

There are other more complicated background subtraction situations faced in SERS experiments where there is not only a variation in the overall intensity but also in the spectral shape. This is the case in single-molecule SERS experiments of resonant or pre-resonant molecules.

When observing many molecules in SERS, the SEF background is typically broad and has an overall shape that does not change by a great deal when focusing the laser in a new location. However, due to the interest in single molecule measurements in recent years [9], we are often forced to treat spectra that can have rapidly varying backgrounds (in both intensity and shape). This is because the dispersion of the plasmon resonance is no longer washed out due to averaging over many molecules. The dispersion and strength of a single plasmon resonance is very dependent on the local conditions of the molecule being observed and, as a result, time series or spatial mappings of single molecule events will typically have very different backgrounds that will often overlap, 


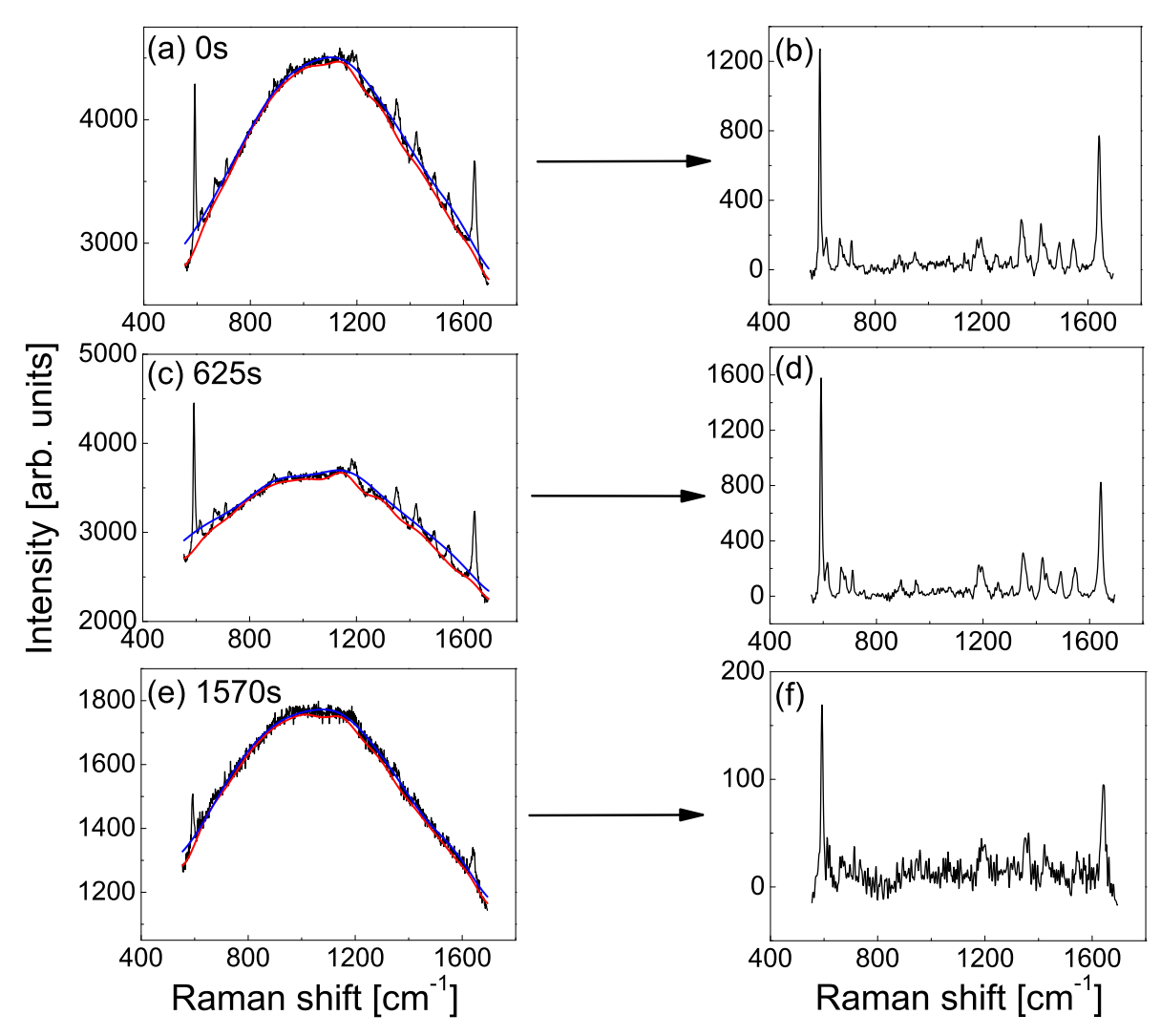

Figure 5.18: The original spectrum, background fits and filtered spectrum for 3 different times. Two background fits are performed: one with the iterative algorithm (red lines), and one using the approximation 7 spectrum of the unmodified signal (blue lines). The chosen spectra were taken at $t=0 \mathrm{~s}$, where the potential difference was positive; $t=625 \mathrm{~s}$, just as the potential was switched to negative and the Raman signal was at a maximum; and $t=1570 \mathrm{~s}$, when the potential was switched to positive and the Raman signal was at a minimum (see reference [160] for further details). 

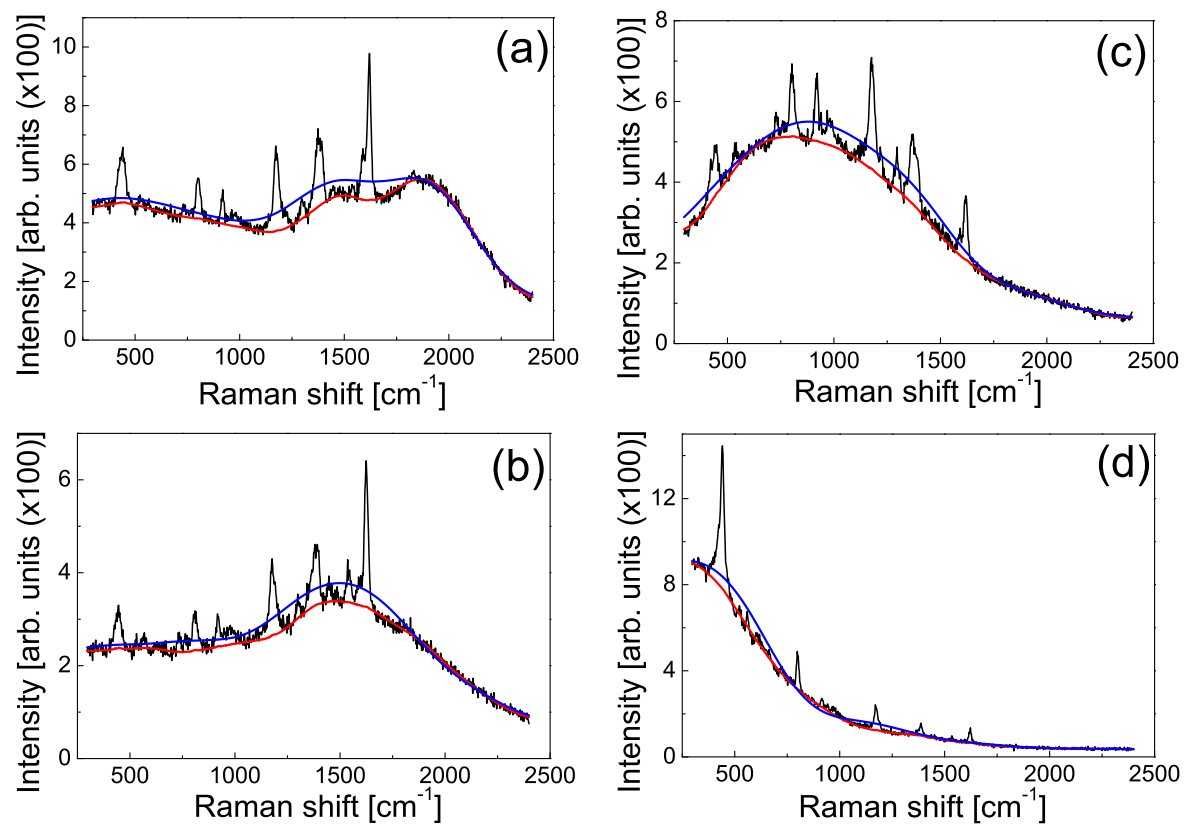

Figure 5.19: Four single molecule SERS events of $C V$ with extremely different SEF backgrounds due to a varying spectral position and width of the plasmon resonance. In each case, two background fits are performed: one using the iterative algorithm and a scaling level of 6 (red line), and one using the approximation 7 spectrum on the unmodified signal (blue line). A background region is defined at each end of the spectra, and between the $441 \mathrm{~cm}^{-1}$ and $803 \mathrm{~cm}^{-1}$ modes, and the iterative fit is performed with the "typical fitting parameters" listed in table 5.3. This figure is adapted from figure 6 in reference [2].

in the frequency domain, with the Raman peaks. The second example will therefore show measurements that look at how the fluorescence background and Stokes SERS intensities vary (relative to each other) for single molecule SERS events. The physical interpretation of these results will be given in the following chapter and only the background removal process will be explained. In this case, the COBRA needs to be able to analyse thousands of spectra without any additional input from the user.

In figure 5.19, four different single molecule events are shown, each with extremely different backgrounds and yet still fit with the same parameters. The frequency components of the background can now be significantly higher than in the previous example as we are observing individual plasmon resonances. As a result, the decomposition can only be performed to 6 levels while keeping the background contribution completely contained within the final approximation 


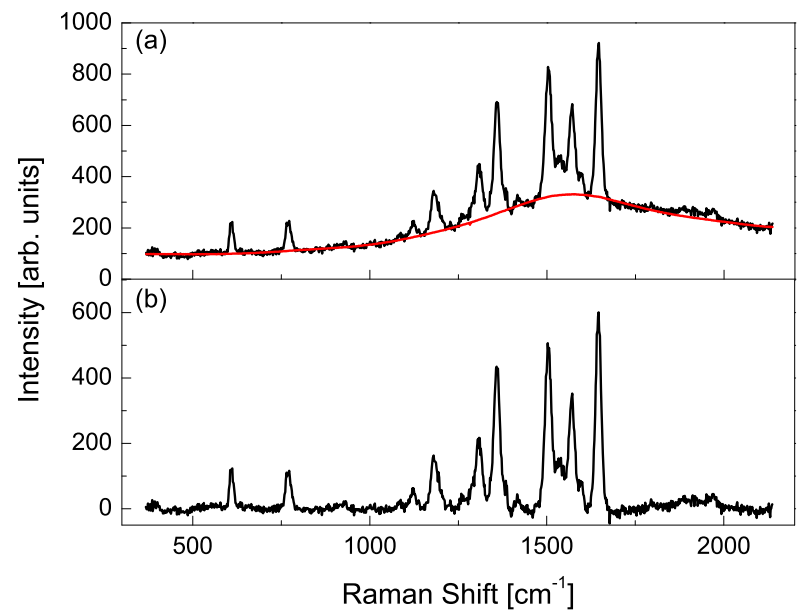

Figure 5.20: (a) A single molecule SERS spectrum of RH6G with a background fitting and (b) the signal after the background has been removed. A decomposition level of 7 and four background regions were used; one at each end of the spectrum, and one between $807 \mathrm{~cm}^{-1}$ and $1052 \mathrm{~cm}^{-1}$, and another between $1689 \mathrm{~cm}^{-1}$ and $1843 \mathrm{~cm}^{-1}$. This figure is adapted from figure 7 in reference [2].

spectrum. Furthermore, an additional background region (other than at the sides of the signal) between the $440 \mathrm{~cm}^{-1}$ and $800 \mathrm{~cm}^{-1}$ modes has to be defined in order for the background fit to converge accurately. This is not required for lower frequency backgrounds, like the ones of the previous example. The predicted background using the non-iterated approximation 7 spectrum has also been plotted in this case to show that the COBRA achieves much more accurate results.

Other measurements using RH6G instead of $\mathrm{CV}$, which has a dense region of modes from $\sim 1100 \mathrm{~cm}^{-1}$ to $\sim 1650 \mathrm{~cm}^{-1}$, have also been investigated and can also be fitted with a great deal of accuracy. Figure 5.20 shows a single molecule RH6G event with a background fitting. Due to the densely packed region of peaks, the decomposition level can not be performed any lower than 7 since the peaks would significantly modify the shape of the fit. As a consequence, additional background regions need to be defined on either side of this region. This example shows that artifacts introduced by closely packed peaks can be reduced by including additional background regions. 


\subsubsection{A Comparison with other Wavelet Based Algorithms}

The iterative algorithm addresses the problem of background removal for large numbers of spectra that contain largely varying backgrounds which often overlap in the frequency domain with the signal peaks. To the best of our knowledge, there are no other wavelet-based algorithms that address this issue entirely. There are, however, several examples of algorithms that partially address the issue.

For example, if there is no overlap in the peak and background frequency components then there are several publications $[156,157]$ that use the high scale approximation spectra as a background fit. This is possible because the signal peak contribution can be removed in the lower level detail coefficients leaving only the background contribution in the approximation coefficients. This can easily be extended to many spectra, even if there is a great deal of variation in the background between events. However, this was not the case for the SERS examples shown in this chapter. In these cases the background fit that would have been achieved using more conventional wavelet-based approaches was also plotted next to the background fit predicted using the iterative algorithm. It was obvious in all cases that the iterative algorithm achieved much better results.

If we only wish to treat a single spectrum then, even if there is an overlap between the frequency regions of the background and peaks, we can use thresholding [143] or the derivative spectrum [142] to remove the background. Both of these techniques are very case dependent however and cannot be generalised to many events.

\subsection{Conclusion}

We have shown that using an algorithm based on WTs and the MPA, we can remove the background from many signal events in which there is a large variation in the background shape and size. Furthermore, the iterative algorithm can accurately treat signals in which there is an overlap in the frequency domain of the peaks and the background. The basis of the iterative algorithm was to perform a signal decomposition using the MPA and then modify the signal based on the final approximation. This has the result that the signal peaks are partially removed without affecting the background. Once the process has been repeated a sufficient number of times, the signal peaks are completely removed and the final approximation spectrum is an accurate background fit. 
With the iterative algorithm at its core, an application (the COBRA) was built for the complete de-noising and background removal of large numbers of spectra. The de-noising was achieved by Fourier transforming the background removed signal and applying a low pass filter. This could also be done using WTs but FTs were preferred due to their flexibility and the fact that noise is better represented by a sine function than a wavelet.

We found that, depending on the distribution of peak sizes and their proximity to each other, we were restricted in the decomposition level that could be performed before the signal peaks introduced artifacts that could not be removed with the iteration number. However, by increasing the number of background regions, the background fit could still be extremely accurate even at relatively large decomposition levels. Furthermore, even when the signal peaks were isolated and narrow, a background region at each end of the signal was typically necessary in order for the fit to converge in that region.

In the next chapter, the algorithm will be applied to measuring the nonradiative enhancement factor. Some of the spectra that were collected for this study have already been shown in figures 5.19 and 5.20. However, the physical interpretation of the results and the reason why the separation of SEF and SERS is so important will be given in section 6.5.

The COBRA application can be downloaded from our lab website [130] along with a manual on how to use the program. 


\section{Chapter 6}

\section{Vibrational Pumping of Single Molecules and Non-Radiative Processes}

\subsection{Introduction}

After a detour in the previous chapter on the development of an analysis tool, we return to one of the pending questions regarding vibrational pumping and the meaning of the pumping cross-section. Prior to the start of my $\mathrm{PhD}$ research, it was believed that the pumping cross-section was the same as the radiative SERS cross-section which is proportional to the observed Stokes intensity. From this cross-section, it was possible to obtain a SERS enhancement factor (by taking the ratio of the SERS cross-section with the bare Raman cross-section of the same molecule), that could be of the order of $10^{12}[8,92]$ (when properly normalized). However, values this high were very difficult to justify with theory and were discounted as a problem with the technique.

The inherent difficulty in measuring SERS cross-sections comes from our inability to estimate the number of molecules contributing to the overall SERS signal. Vibrational pumping avoids this problem by taking the ratio of the Stokes and anti-Stokes intensity which are both linearly dependent on the number of molecules.

The detection of single molecule SERS has given us a tool for measuring the 
scattering intensity of a single molecule which can then be converted into a crosssection by comparing it with a reference molecule with a known cross-section (see appendix C). The largest cross-sections measured from single molecule events were found to correspond to SERS enhancement factors of $\sim 10^{10}-$ $10^{11}$, which is $1-2$ orders of magnitude smaller than what can be found with vibrational pumping. It is not obvious, however, whether vibrational pumping can be observed under single molecule conditions as we need to be able to detect anti-Stokes scattering of molecules with extremely small vibrational populations. The plasmon resonance producing the signal must therefore be large enough to enhance the anti-Stokes signal such that it is larger than the noise level.

In this chapter, I will demonstrate that it is indeed possible to observe vibrational pumping of single molecules, and that a comparison can be performed between the SERS and pumping cross-sections. I will also show that the pumping cross-section will typically get smaller as the SERS cross-section increases as a result of non-radiative effects and the asymmetry factor, which will provide strong evidence that the cross-section measured using vibrational pumping, is not the radiative SERS cross-section previously assumed. I will also include an investigation into the non-radiative enhancement factor, by way of simultaneous measurements of SEF and SERS, that will demonstrate that the total enhancement factor (non-radiative + radiative) is much larger than the radiative enhancement factor.

\subsection{Modification of Pumping Theory under Sin- gle Molecule Conditions}

In chapter 4, I gave an in depth review of vibrational pumping for large numbers of molecules. In this chapter I will demonstrate how the theory is modified when we move to a regime in which single molecules are measured. It was shown that we can measure the pumping cross-section of a single Raman active mode from the ratio of the anti-Stokes and Stokes intensities, at low temperatures, via:

$$
\rho=\frac{I_{a S}}{I_{S}}=A^{E} \frac{\tau \sigma_{\text {pump }} P_{L}}{\hbar \omega_{L}},
$$

where $\sigma_{\text {pump }}$ is the pumping cross-section and given by:

$$
\sigma_{\text {pump }}=\frac{\left\langle\sigma_{S}^{T} d \sigma_{a S}^{R} / d \Omega\right\rangle}{\left\langle\sigma_{a S}^{R} / d \Omega\right\rangle} .
$$


where $\sigma_{S}^{T}$ is the total Stokes cross-section (not differential) and $d \sigma_{a S}^{\mathrm{R}} / d \Omega$ is the radiative anti-Stokes differential cross-section. The asymmetry factor, $A^{E}$, in equation 6.1 was defined in section 4.2.1 as:

$$
A^{E}=\frac{\left\langle d \sigma_{a S}^{R} / d \Omega\right\rangle}{\left\langle d \sigma_{S}^{R} / d \Omega\right\rangle},
$$

It is important to distinguish the radiative cross-sections from the total crosssection because a fraction of the Stokes scattered photons will be absorbed by the metallic substrate and, as a result, will not be detected by the spectrometer. The triangular brackets correspond to an averaging of the variable inside the brackets over all molecules that are measured.

In the single molecule regime, the distribution of enhancement factors can be ignored as we are measuring the absolute cross-sections for a single molecule and not a spatial averaging of many. The anti-Stokes to Stokes intensity ratio therefore simplifies to:

$$
\rho=A \frac{\sigma_{\mathrm{pump}} \tau P_{L}}{\hbar \omega_{L}} .
$$

where the asymmetry factor, $A$, is now for a single molecule and not an ensemble average.

A key advantage of performing pumping measurements under single molecule conditions, is that the differential radiative SERS cross-section can be measured simultaneously with the pumping cross-section by comparing the Stokes intensity with a reference compound that has a known Raman cross-section (see appendix C). This is a common technique which has been utilized in the past [161] and the relationship between these two important cross-sections will be explored later in this chapter. Compared to measurements of large numbers of molecules, the pumping cross-section will be slightly larger as only those molecules with the largest enhancement factors will contribute to the signal. Both the pumping and SERS cross-sections will depend strongly on the local environment of the molecules being measured. Even a difference of a couple of nanometers in the location of a molecule close to a hot-spot can result in a substantial difference in the local field enhancement factor (see section 3.3.2). As a result, at large concentrations, there will be an extremely large number of molecules receiving relatively low enhancements but still contributing significantly to the Stokes intensity but not the anti-Stokes (see section 4.2.1). Consequently, the pumping cross-section is slightly underestimated and we can only measure a lower limit 
for high concentrations.

Equation 6.4 has the additional advantage that it is independent of the effects of photo-bleaching [102]. Because the anti-Stokes and Stokes intensities are modified equally by the lifetime of the molecule, taking the ratio of the intensities results in a quantity that is constant with integration time. This is a significant advantage as photo-bleaching is often a problem when considering single molecule SERS measurements and vibrational pumping, in particular, due to the relatively large power densities and enhancement factors required to observe the effect. It is important to note that this is different to the effect that photo-bleaching has on large numbers of molecules (see section 4.2.7).

In section 4.2.6 I discussed a technique that we developed for estimating the vibrational lifetime. A source of inaccuracy in the estimates was described as being due to inhomogeneous broadening [10] resulting from small shifts in the peak location for different molecules that have different local environments. By going to a single molecule regime, we have avoided this problem and the only source of error should be due to the dephasing lifetime (discussed in section 4.4).

In the case of many molecules, the asymmetry factor is spatially averaged along with the cross-sections (see equation 6.1) which has a significant impact on the estimate of the pumping cross-section. As explained in section 4.2.4, the asymmetry factor will typically depend on two effects; the plasmon resonance of the particles creating the enhancement factor, and the electronic resonance of the molecule being observed. Each of these will enhance the Stokes and anti-Stokes scattered photons of a single mode differently and, hence, create an asymmetry between their cross-sections. Because the dispersion (wavelength dependence) of the plasmon resonance is dominated by the properties of the metallic particles, the contribution to the asymmetry factor for each molecule arising from this effect will be different and depend on the local environment of that particular molecule. Figure 6.1 shows the dispersion of the plasmon resonance for a single molecule under two different excitation wavelengths (Note: this is an idealised case). When using a high energy excitation (the left side of the peak), the Stokes scattered photons receive a larger enhancement than the anti-Stokes scattered photons. Ignoring other effects, this will result in a asymmetry factor that is less than one, for this particular molecule. If we use a low energy excitation on the other hand, such that we are on the right side of the peak, anti-Stokes scattered photons will receive the larger enhancement and the asymmetry factor will be large than one. The exact dispersion of the plasmon 


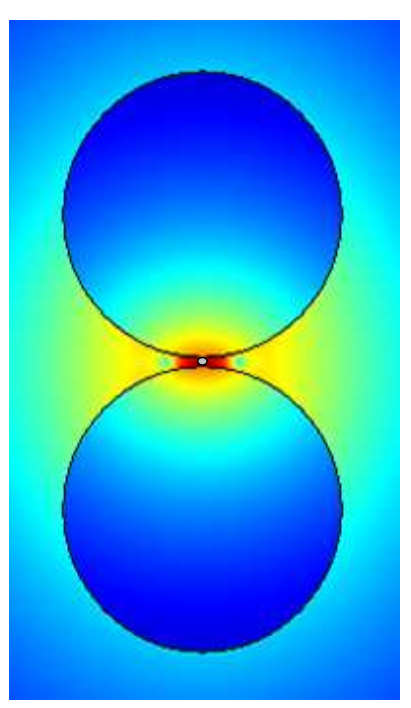

\section{Plasmon Resonance Dispersion}

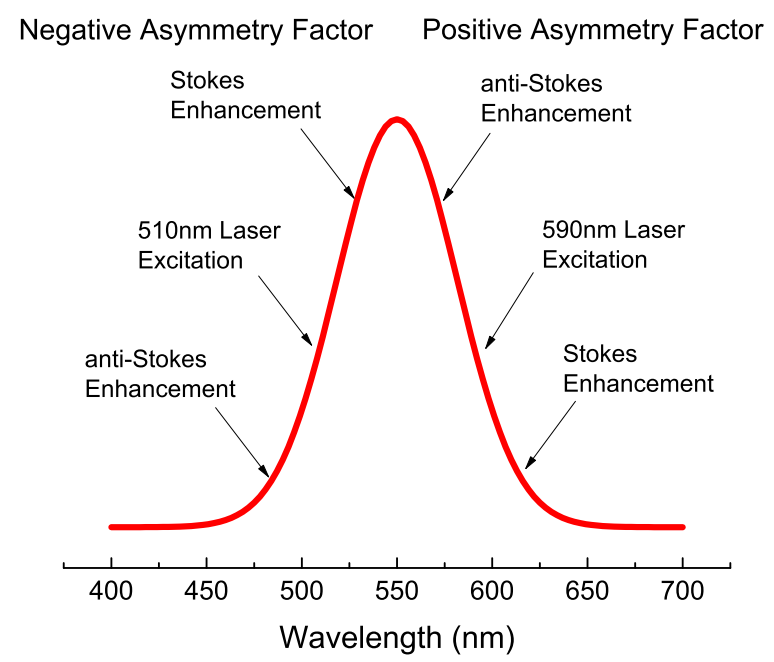

Figure 6.1: The dispersion of an idealized plasmon resonance in the gap region of a dimer (see chapter 3.3.2). At a high energy excitation (the left side of the peak) the Stokes photons receive a larger enhancement than the anti-Stokes photon which results in a small asymmetry factor. For a low energy excitation (the right side) the opposite happens and we have a large asymmetry factor.

resonance will depend greatly on where the molecule is located and hence the asymmetry factor arising from this effect will vary greatly. Furthermore, the dispersion of the plasmon resonance at a hot-spot can be extremely sharp and result in asymmetry factors that can range from 0.1 to 10 .

The electronic resonance of the molecule will similarly create an asymmetry as either the Stokes or anti-Stokes wavelength will be closer to the absorption resonance of an electronic transition. However, because this is a property of the molecule itself and not its environment, this asymmetry should be approximately constant for all molecules excited at the same wavelength. The physical origin of the asymmetry factor was discussed in section 4.2.4.

In the many molecule case it is obvious that the asymmetry from the electronic resonance will be the same as in the single molecule case, however, the asymmetry from the plasmon resonance will now be a spatial average over all molecules. One might expect that for a large enough number of molecules, the plasmon resonance contribution will eventually average to one. However, this is generally not the case, largely due to the dielectric function of the substrate 
(see section 3.1.3), and the distribution of particles sizes and separations. For $\mathrm{Ag}$, the plasmon resonances around $350 \mathrm{~nm}$ to $400 \mathrm{~nm}$ will be the strongest and the average dispersion in the plasmon resonances will likely reach a maximum somewhere in this range as long as there are sufficient hot-spots with resonances in this region. The key point is that due to the spatial averaging, the asymmetry resulting from the plasmon resonance will be much smaller in the many molecule case and, hence, when considering single molecule events, we must estimate $A$ on a case by case basis.

Later in this chapter I will investigate how much the asymmetry factor can vary for single molecules and how it affects our estimate of the pumping crosssection.

\subsection{Initial Observations of Single Molecule Vi- brational Pumping}

In this chapter I will give an overview of the experimental results for single molecule vibrational pumping which will eventually lead to a rigorous statistical investigation into the physical meaning behind the pumping cross-section and the effects of the asymmetry factor. However, understanding the early measurements and their puzzling results is necessary in order to appreciate the difficulty in the interpretation. Before I can discuss the early results, it is important that I give a brief introduction to single molecule SERS $[162,163]$.

\subsubsection{The Two Analyte SERS Technique}

The bi-analyte SERS (BiASERS) technique was pioneered here at Victoria University by Le Ru et al. [109] in which large numbers of SERS measurements were taken of solutions containing two SERS analytes with distinguishable Raman spectra [164]. In order to obtain the cross-sections necessary to observe single molecules, analytes were chosen that had an absorption resonance close to the laser excitation (resulting in a large Raman cross-section) and added to a solution of aggregated $\mathrm{Ag}$ colloids (see section 4.3.3). SERS spectra were then taken as a function of time, in the case of a liquid sample, or space, for a dry sample.

For a typical BiASERS experiment, we will observe three different types of spectra as shown in figure 6.2; events dominated by one analyte or the other (see Fig. 6.2(a) and (b)) and events that contain a mix of both (see figure 6.2(c)). 

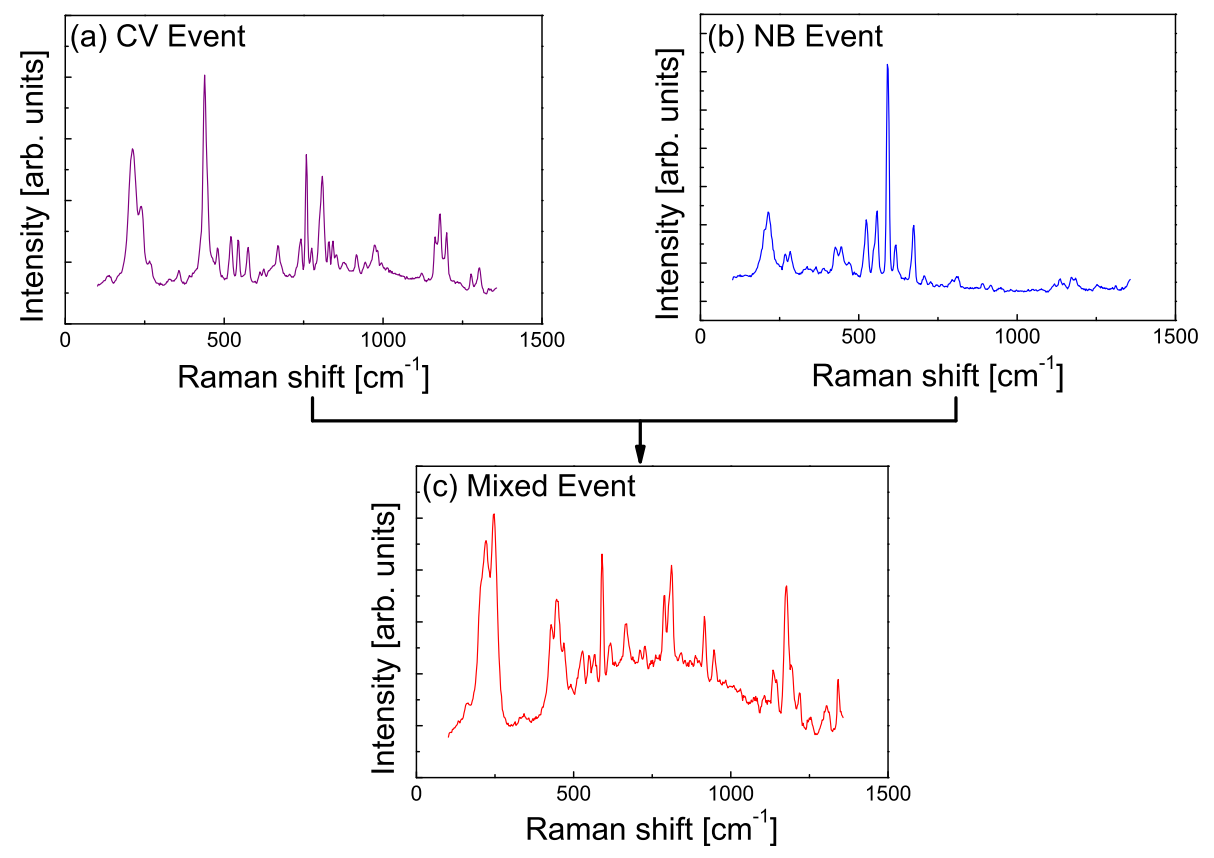

Figure 6.2: The three different types of spectra observed in BiASERS of $C V$ and NB; (a) events dominated by the CV Raman spectrum, (b) events dominated by the NB Raman spectrum and (c) events with a significant contribution from $C V$ and $N B$. Depending on the relative number of events of each type, it is possible to make conclusions about the single molecule nature of the experiment. 
Using principal component analysis [165] (or a linear decomposition) over a region small enough that the shape of the plasmon resonance is not important, it is possible to decompose all of the events into a linear combination of the Raman spectra for each analyte:

$$
S_{T}(i i)=c_{1}(i i) S_{1}+c_{2}(i i) S_{2},
$$

where $S_{1}$ and $S_{2}$ are the Raman spectra for analyte 1 and 2 respectively, $S_{T}$ is the total signal, and $i i$ is the event number. A histogram can then be made of the ratio $c_{1} /\left(c_{1}+c_{2}\right)$ for events with a sufficiently large intensity. A ratio of 0 will correspond to an event which is completely dominated by the second analyte, and a ratio of 1 will correspond to an event that is completely dominated by the first analyte. Any ratio between 0 and 1 will be a mixed event with traces of both molecules. To make the argument that we are in a single molecule regime, we need to have a histogram that has a large number of events with a ratio of 0 or 1 compared to mixed events.

If we have a sample where there are many colloidal aggregates within the scattering volume of the laser, then there will likely be several hot spots. The concentration must therefore be low enough that only a total of 1 or 2 molecules will be in hot-spots as shown in figure 6.3. In this example, the concentration is small enough that there is only one molecule within a hot spot and, as a result, the SERS spectrum for this event will be completely dominated by that molecule. There is a second molecule within a gap (where hot-spots form) but as it is outside of the scattering volume, it will not contribute to the SERS signal. If we take many events at this concentration, we would expect that most will be single molecule. However, if the concentration is increased, we will eventually reach a regime where there are typically many molecules in hot spots and few single molecule events.

In a dry sample, it is mainly the concentration that dictates the single molecule nature of the SERS experiment as there is no movement of the colloids within the scattering volume. A liquid sample on the other hand has colloids that move with Brownian motion in and out of the laser spot. If we go back to our example shown in figure 6.3 , it is possible at large enough integration times for the aggregate with the second molecule in the gap to move into the scattering volume. This will result in a mixed event instead of the single molecule event measured with a dry sample. For a liquid sample, our ability to detect single molecule events will therefore depend on both the concentration and integration 


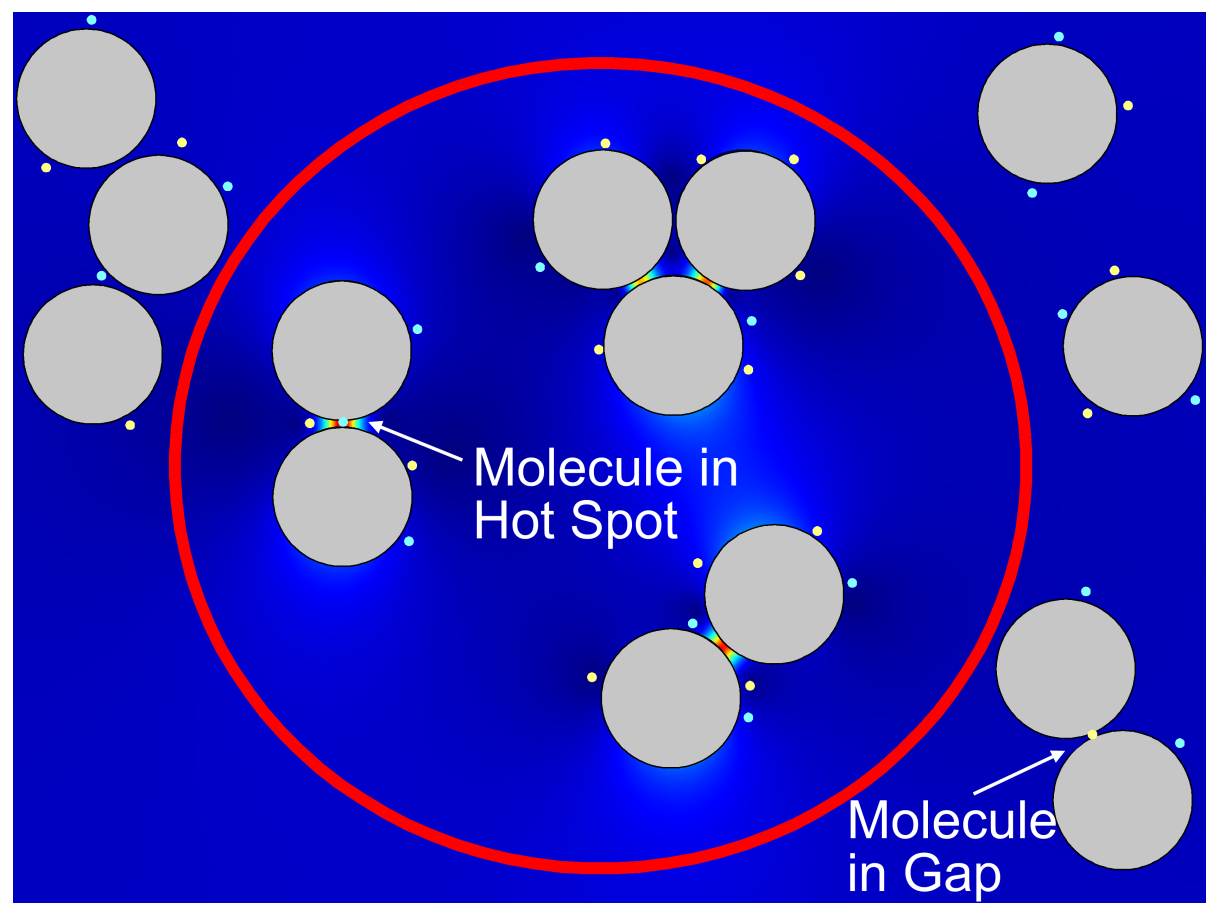

Figure 6.3: A diagram of a simplified BiASERS event in which there are three colloidal aggregates within the scattering volume (the red circle) and several aggregates outside. The small blue and yellow circles correspond to the two analytes being probed. The background colour is a log scale for the electric field intensity in which the blue regions are low intensity and the red regions are high. In this case, the concentration is low enough that there is only one molecule in a hot spot (region with extremely high field intensity). There is a second molecule within a gap outside the scattering volume. 


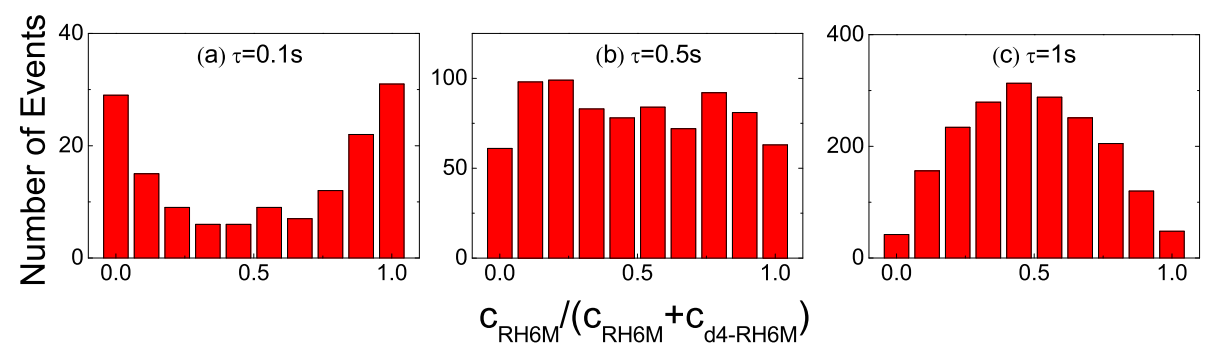

Figure 6.4: Histograms showing how the number of events with different ratios of $c_{1} /\left(c_{1}+c_{2}\right)$ varies with integration time for a liquid sample of deuterated RH6M(d4RH6M) and non-edited RH6M. This figure is adapted from figure 6 in reference [166].

time. Figure 6.4 shows how the histogram varies as a function of integration time for two Rhodamine $6 \mathrm{M}$ molecules where one has been isotopically edited, substituting some of the hydrogen nuclei for deuterium [166,167]. At an integration time of $0.1 \mathrm{~s}$, there are many events with a ratio of 0 or 1 compared to mixed events. As a result, we can assume any spectrum containing only the Raman spectrum of one analyte will be a single molecule event. For $0.5 \mathrm{~s}$, the histogram is reasonably flat and the single molecule nature of the single analyte events are very difficult to distinguish. Once the integration time has been increased to $1 \mathrm{~s}$, there are very few events that contain one analyte. The concentration of the dye was $1 \mathrm{nM}$.

Typically, liquid samples provide fewer problems when performing single molecule measurements as it is difficult to prepare dry samples with uniformly spaced colloidal aggregates. However, due to the low temperatures required for vibrational pumping, dry samples are needed. Using dry samples gives us the advantage that we can perform time resolved measurements of single molecule events which cannot be achieved with a liquid sample.

\subsubsection{Experimental Observations of Single Molecule Vi- brational Pumping}

In chapter 4 I discussed how vibrational pumping can be utilised for the estimation of pumping cross-sections. However, under many molecule conditions, it was extremely difficult to confirm experimentally that the cross-sections that were measured agreed with SERS cross-sections measured using a different technique. This problem stems from the fact that we do not know how many 
molecules are contributing to the SERS signal. Using BiASERS we can distinguish single molecule events and measure the cross-section using two techniques; by comparing the Stokes intensity to a reference molecule with a known cross-section (see appendix C), and using vibrational pumping.

For this experiment we chose to use a dry sample consisting of RH6G and $\mathrm{NB}$ to a final concentration of $1 \mathrm{nM}$ each and aggregated $\mathrm{Ag}$ colloids. The solution was dried for 5 minutes using the technique described in section 4.3.3. This resulted in a low density of colloidal aggregates such that there are only a few hot-spots created within the scattering area $(\sim 1.5 \mu \mathrm{m}$ in diameter). The sample was mounted on the LabRam system (see section 4.3.1) and cooled to a temperature of $77 \mathrm{~K}$. SERS spectra were taken using the $\times 50$ long working distance objective, a 300lines $/ \mathrm{mm}$ grating and an excitation of $1.7 \times 10^{8} \mathrm{~W} / \mathrm{m}^{2}$ at $633 \mathrm{~nm}$. Spatial mappings were performed with an integration time of $1 \mathrm{~s}$ for a total of $\sim 6000$ spectra.

The peaks that were monitored were the $610 \mathrm{~cm}^{-1}$ of RH6G and $595 \mathrm{~cm}^{-1}$ of NB. Figure 6.5 shows selected spectra with different levels of pumping; (a) Stokes scattering of both dyes but no anti-Stokes scattering, (b) anti-Stokes and Stokes scattering of both dyes, (c) and (e) Stokes scattering of one dye or the other, (d) and (f) Stokes and anti-Stokes scattering of one dye or the other. It is obvious that (a) and (b) cannot be single molecule events but at these concentrations (c) to (f) likely are. The fact that there is an anti-Stokes peak in (d) and (f) means that we have pumping of single molecules. The question then arises, why is there not an anti-Stokes signal in all of the events? It is obvious from figure 6.5 that even for large Stokes events, observing vibrational pumping through anti-Stokes scattering is not always possible. It is therefore not enough for the local field enhancement to be extremely large, we must also have a plasmon resonance that favours anti-Stokes scattering.

Figure 6.6 shows single molecule SERS spectra taken at a very low power densities $\left(\sim 1.7 \times 10^{6} \mathrm{~W} / \mathrm{m}^{2}\right)$ for four different plasmon resonance dispersions. The low power was necessary in order to obtain the best resolution of the plasmon resonance. The scattering enhancement favours the medium, high and low energy modes in figure 6.6(a), (b) and (c), respectively, whilst in (d) all of the modes are enhanced similarly. Figure 6.6(c) also contains a signal on the antiStokes side which can be confused with pumping but which is likely due to a thermal population in the lowest energy modes. It is obvious from these spectra that the plasmon resonance experienced by a single molecule can significantly favour a small range of vibrational modes. As a result, the asymmetry factor 

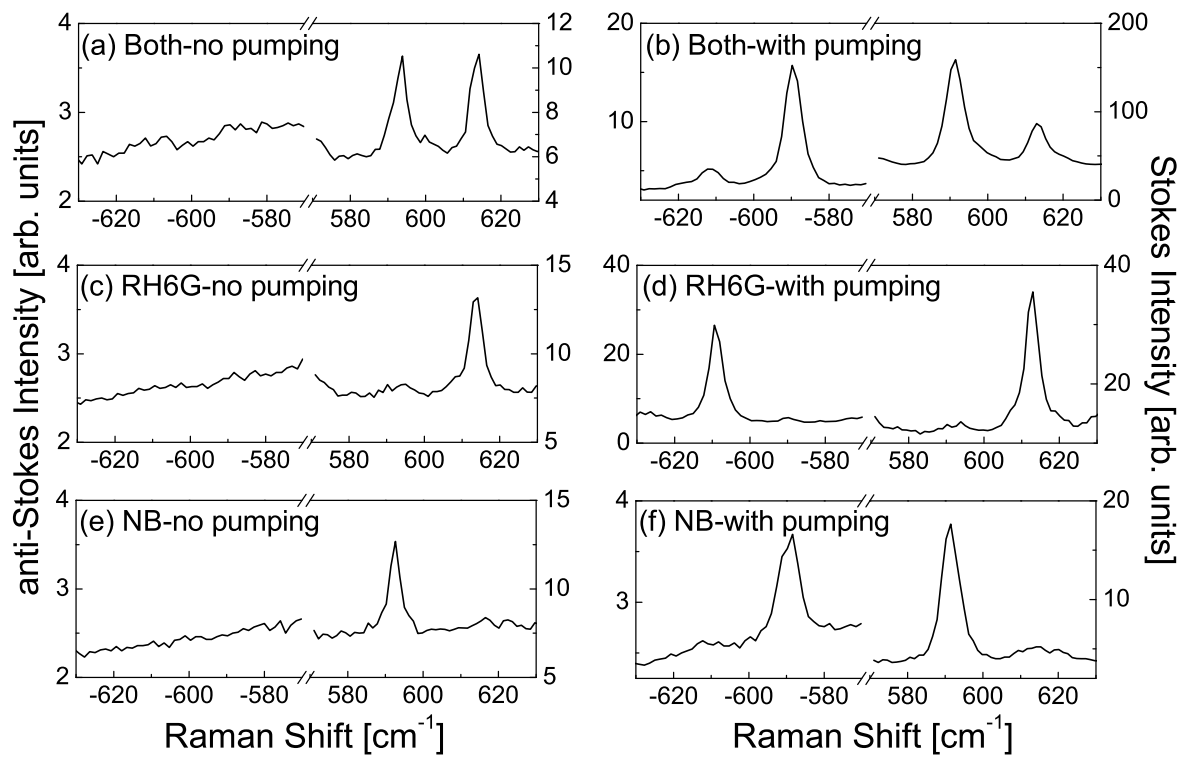

Figure 6.5: Selected BiASERS spectra taken at $77 \mathrm{~K}$ where anti-Stokes scattering will only be observable if vibrational pumping is present. (a) and (b) show mixed events where both dyes are present and pumping is observed in one case but not the other. (c) and (e) show single molecule events of one dye or the other but without pumping (no anti-Stokes scattering) while (d) and ( $f$ ) show single molecule events with pumping (both anti-Stokes and Stokes scattering is observed). 

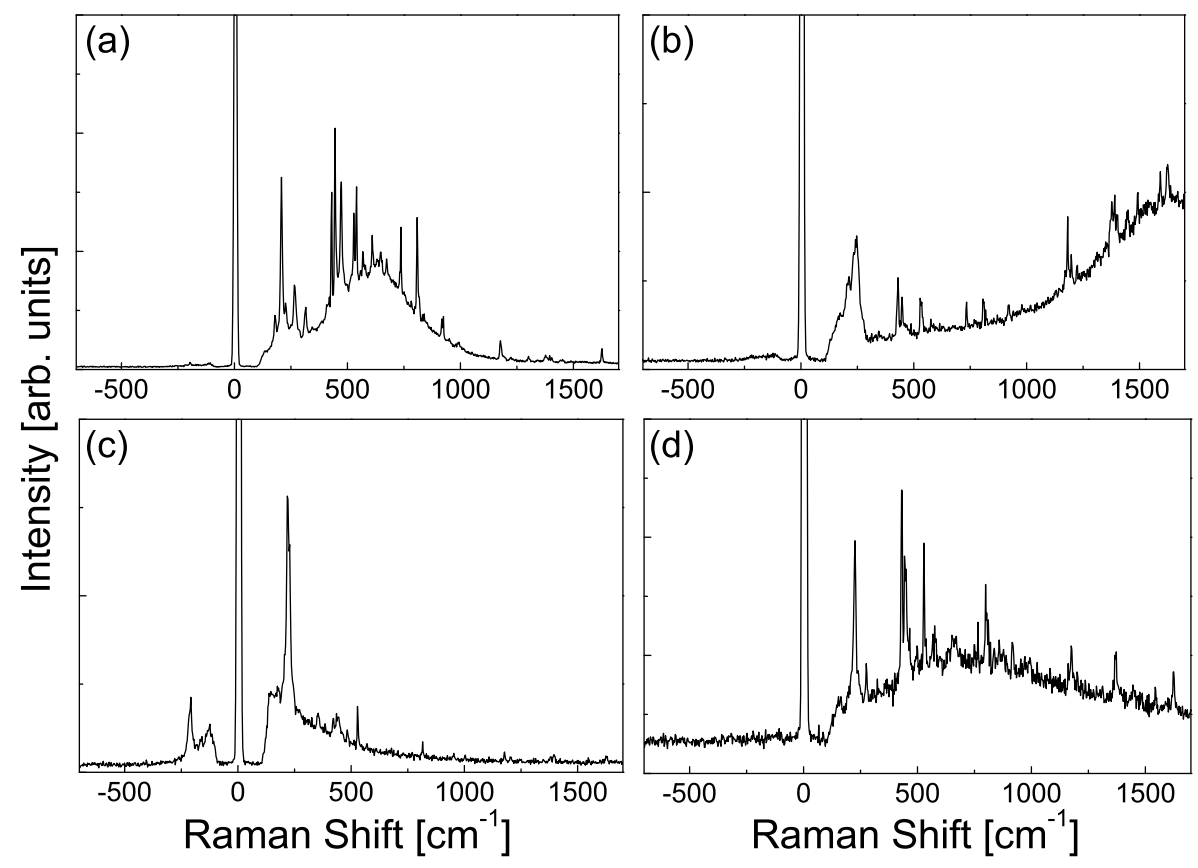

Figure 6.6: Four single molecule SERS spectra with different plasmon resonances which mostly enhance the (a) medium energy modes, (b) high energy modes, (c) low energy modes as well as anti-Stokes scattering, and (d) all of the modes. These spectra are taken at an extremely low power $\left(\sim 1.7 \times 10^{6} \mathrm{~W} / \mathrm{m}^{2}\right)$ in order to resolve the resonance. This figure is taken from figure 11 in reference [92].

can vary dramatically between events. There are also many spectra that contain no recognizable signal as there are no molecules close to hot-spots that are sufficiently strong, being excited.

An additional test to support the assumption that we are in fact observing single molecules is to perform time dependent measurements at a single location. Because the sample is dried on a substrate, there will not be any movement of the molecules and colloids from the scattering area except for small thermally activated movements or photo-bleaching. As a result, if we focus the laser at a position on the substrate where single molecule pumping is apparent and perform a time series mapping, the Stokes and anti-Stokes intensity should be approximately constant until, at some time, the molecule photo-bleaches and the signal completely disappears simultaneously on both sides. Figure 6.7 is an example of a NB pumping event where the molecule photo-bleaches sometime between 17 and $18 \mathrm{~s}$ after first being first observed. Ideally we would like to 
Figure 6.7: $\quad$ Time dependence spectra of the $595 \mathrm{~cm}^{-1}$ mode of $N B$ taken every $1 \mathrm{~s}$ observing both the anti-Stokes and Stokes signal under single molecule pumping conditions. The anti-Stokes and Stokes intensity remains approximately constant until the molecule photo-bleaches after $17 \mathrm{~s}$ at which time the peaks simultaneously disappear.

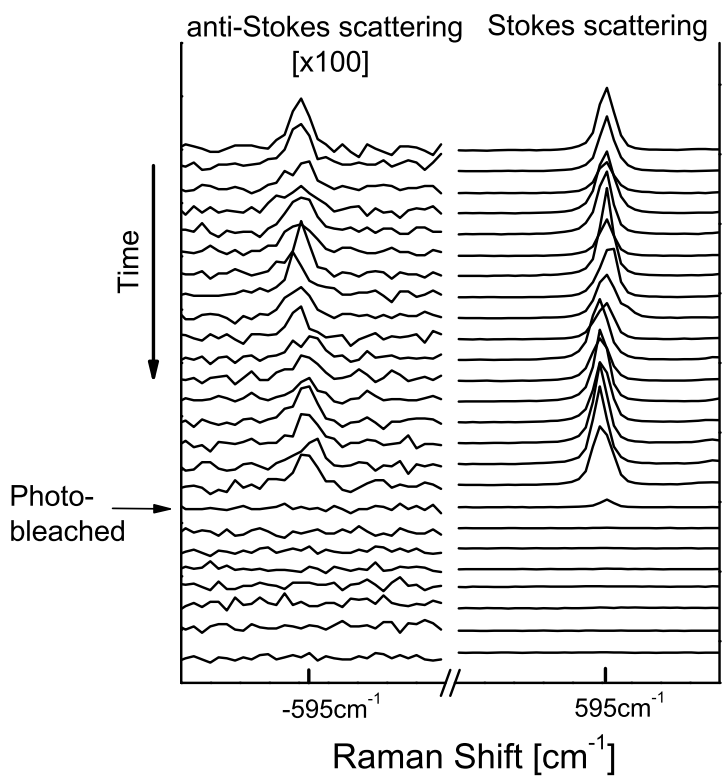

perform this test for each possible single molecule event. However, if we wish to take large numbers of single molecule events, so that a statistical analysis can be performed, this is not possible. Furthermore, simply observing the disappearance of the signal is not a confirmation of single molecule pumping as slight movements of the substrate can also explain these results. Nevertheless, if single molecules are being observed, these types of spectra are expected. It is our belief, that the BiASERS results coupled with the time dependent measurements are extremely strong evidence for the existence of single molecule pumping.

Once the single molecule pumping events had been identified, it was possible to calculate the SERS and pumping cross-section for each. The SERS cross-section was estimated by comparing the Stokes intensity with the Stokes intensity of Nitrogen taken under the same experimental conditions (see appendix C) whilst the pumping cross-section was estimated using equation 6.4 (with a $\tau$ of $1 \mathrm{ps}$ ). It was found that the pumping cross-section could be as much as two orders of magnitude larger than the Stokes cross-section. This is too large a discrepancy to explain with the asymmetry factor which could at most explain a difference of a factor of 10 with a factor of 5 being more likely. 


\subsection{A Statistical Analysis of the Pumping Cross- section}

It is apparent from the early measurements that the pumping cross-section is not the same as the SERS cross-section as has been assumed in the past $[4,92]$. However, because there were very few examples of single molecule pumping, it was unclear how the pumping cross-section varied and whether it was dependent on the SERS cross-section. Furthermore, it was not obvious how important the asymmetry factor was for the detection of single molecules. In order to address these issues, statistics from a large number of single molecule pumping events are necessary. However, several modifications had to be made to the sample preparation before reliable statistics could be obtained.

\subsubsection{Experimental Method}

In the previous section we used a solution consisting of RH6G and NB with a laser excitation at $633 \mathrm{~nm}$. However, at this wavelength, NB is very close to resonance conditions (absorption maximum at $628 \mathrm{~nm}$ [126]) whilst RH6G was significantly off-resonance (absorption maximum at $530 \mathrm{~nm}$ ). As a result, the signal from NB was typically much larger than for RH6G which meant that single molecule pumping events of RH6G were much fewer than NB. This is because the enhancements and asymmetry factors required to observe antiStokes scattering of RH6G were much more restricted. We therefore chose to use CV for our second dye which has a resonance at $590 \mathrm{~nm}$ and, hence, will have a much more comparable signal to NB at this excitation. The reason for our choice of $\mathrm{CV}$ for the single molecule measurements will become clearer in section 6.5. There is a drawback to using CV instead of RH6G as RH6G has a large peak very close to one of $\mathrm{NB}$ (the $610 \mathrm{~cm}^{-1}$ mode of RH6G and the $592 \mathrm{~cm}^{-1}$ mode of NB) whilst CV does not. The closest mode of CV to the $592 \mathrm{~cm}^{-1}$ of NB is the $802 \mathrm{~cm}^{-1}$ mode which is a difference of $\sim 200 \mathrm{~cm}^{-1}$. This is a large enough region for the dispersion in the plasmon resonance to create difficulties in the principle component analysis linear decomposition [109, 165].

We also found that our sample preparation procedure resulted in spatial mappings (consisting of $\sim 3000$ spectra) with only 4-5 single molecule pumping events due to the large distances between colloidal aggregates. We therefore adjusted the drying time from 5 minutes to 30 minutes. Observing the sample through the spectrometer camera confirmed that the colloidal aggregates were 

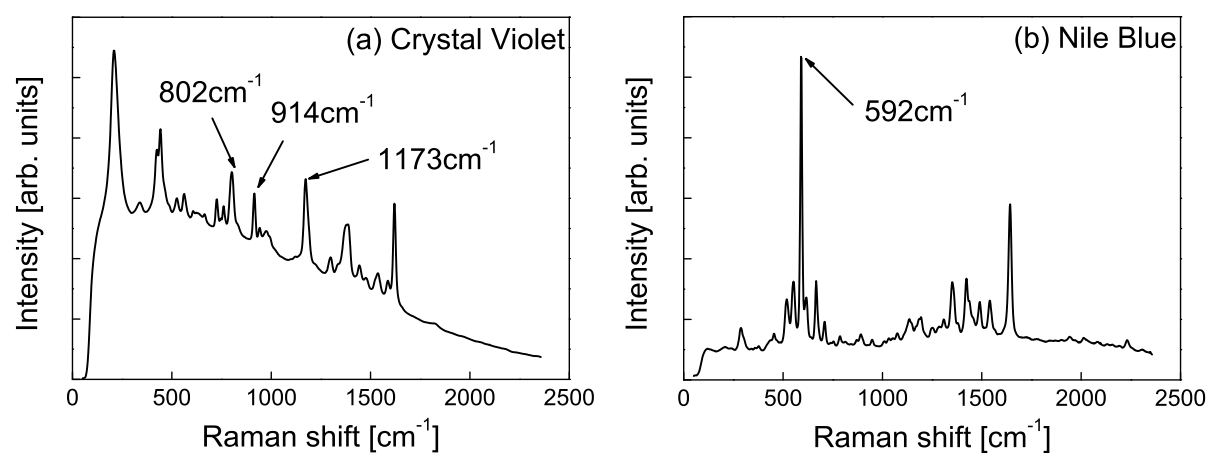

Figure 6.8: Typical SERS spectra for (a) $C V$ and (b) NB. The observed modes are the $802 \mathrm{~cm}^{-1}, 914 \mathrm{~cm}^{-1}$ and $1173 \mathrm{~cm}^{-1}$ peaks of $C V$ and the $592 \mathrm{~cm}^{-1}$ peak of $\mathrm{NB}$. This figure is taken from figure 1 in reference [3].

much closer but had not changed significantly in size. The final concentration of the dyes were $1 \mathrm{nM}$ each.

Using the same experimental set-up as in section 6.3, spatial mappings were performed with a spectrum taken every $2 \mu \mathrm{m} \times 2 \mu \mathrm{m}$ for a total of $6400 \mu \mathrm{m}^{2}$, an excitation power of $2.8 \times 10^{8} \mathrm{~W} / \mathrm{m}^{2}$ and a $0.2 \mathrm{~s}$ integration time.

\subsubsection{Single Molecule Events}

Careful consideration has to be given when deciding which peaks to measure. Typical SERS spectra for CV and NB are shown in figure 6.8. The peaks measured must be at a high enough energy that the vibrational population created through pumping is much larger than that created through thermal excitation at $77 \mathrm{~K}$. Furthermore, both the anti-Stokes and Stokes scattering of all the modes need to be contained within the spectral window of the 300 lines $/ \mathrm{mm}$ grating at $633 \mathrm{~nm}$ (between $-1300 \mathrm{~cm}^{-1}$ and $1300 \mathrm{~cm}^{-1}$ ) and the bare crosssection needs to be large enough that anti-Stokes scattering can be observed regularly. We therefore chose to monitor the $802 \mathrm{~cm}^{-1}, 914 \mathrm{~cm}^{-1}$ and $1173 \mathrm{~cm}^{-1}$ modes of $\mathrm{CV}$ and the $592 \mathrm{~cm}^{-1}$ mode of NB.

The adjustment to the drying time of the sample had the intended result that there were many more single molecule events for each spatial mapping $(\sim 100)$. However, as previously, not all single molecule events contained pumping and, in fact, some events contained anti-Stokes scattering of one dye but Stokes scattering of both dyes. Figure 6.9 contains a two dimensional contour plot 
of a single mapping in which red corresponds to a large, and blue to a small, overall scattering intensity. It is obvious that there are many regions of high enhancement, a few of which are large enough to observe vibrational pumping. The anti-Stokes spectrum at five different locations on the sample (two of which contain the same type of pumping) are also included, demonstrating the four different levels of pumping: (a) pumping of both dyes, (b) and (c) pumping of one dye or the other, and (d) no pumping of either dye.

It is not uncommon that an event with anti-Stokes scattering of a single dye could also have Stokes scattering of both (see figure 6.10). The reason for this lies in the origin of the anti-Stokes and Stokes signal under pumping conditions. In section 4.2.1 I explained that the anti-Stokes signal is proportional to $M_{\mathrm{Loc}}^{3}$ while the Stokes signal is dependent on $M_{\mathrm{Loc}}^{2}$. As a result, the anti-Stokes signal is much more sensitive to the local field enhancement than the Stokes signal. If we consider a scenario in which there are many hot-spots in the scattering area, with a molecule in each, but only one large enough to observe pumping (and with a suitable asymmetry factor), we would expect to see a SERS spectrum with a mixed signal on the Stokes side and only one dye on the anti-Stokes side. A situation like that in figure 6.9(d), where there is an extremely large Stokes signal but no anti-Stokes, can also be explained with many hot spots but with none large enough to observe pumping. Vibrational pumping, therefore, provides an additional tool for understanding the microscopic origin of the signal.

Taking a more quantitative example, let us consider a scenario where there are ten hot-spots within the scattering area of the laser with a molecule in each (five of each dye), but with one hot spot having a local field enhancement that is a factor of 5 larger than the others. If the molecule in this large hot-spot is NB, the Stokes signal of NB will be a factor of $3\left(=\left(5^{2}+9 \times 1^{2}\right) / 10\right)$ larger than that of CV (assuming that the bare cross-sections are the same) and the overall event will be perceived as being a mixed event. The anti-Stokes signal of $\mathrm{NB}$, however, will be a factor of $13\left(=\left(5^{3}+9 \times 1^{3}\right) / 10\right)$ larger than that of $\mathrm{CV}$. The single molecule nature of the event will therefore depend on which type of scattering is being observed.

In section 6.4.1 I mentioned the difficulties in using PCA (principal component analysis) for comparing peaks of $\mathrm{CV}$ and NB. To distinguish single molecule events, we therefore chose to perform PCA on the anti-Stokes side of the spectrum in the range $-850 \mathrm{~cm}^{-1}$ to $-550 \mathrm{~cm}^{-1}$ which encompasses the two closest modes of each molecule. Performing PCA on the anti-Stokes signal has the advantage that the background is relatively flat due to the lack of fluo- 


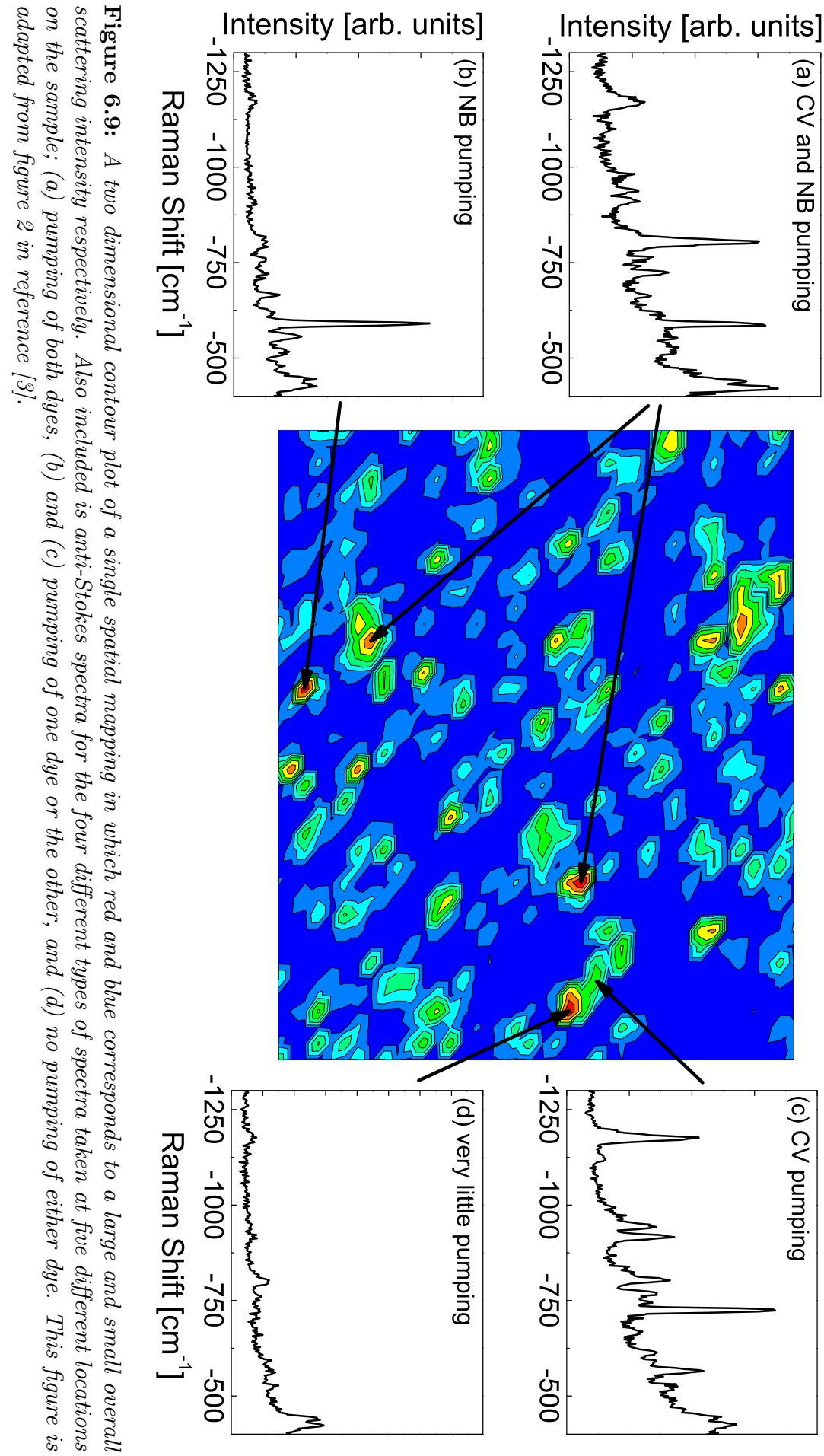




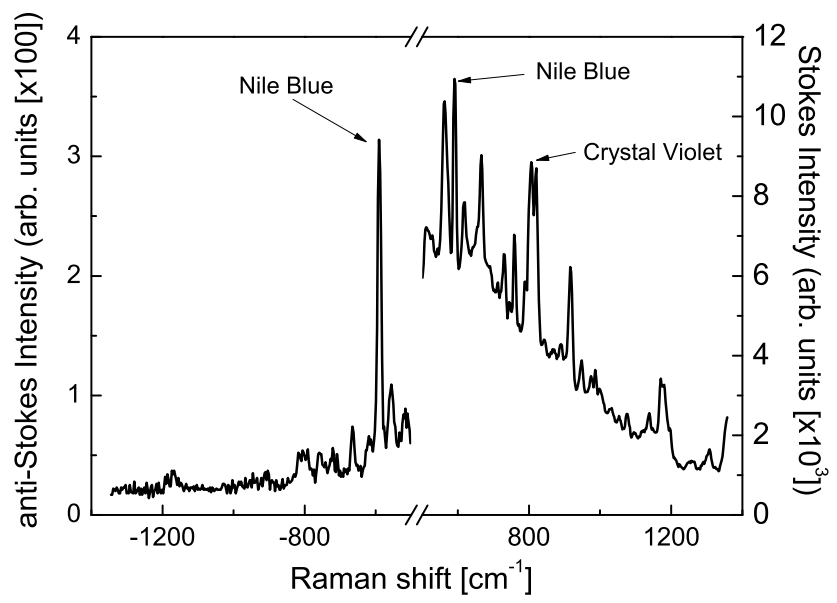

Figure 6.10: A SERS spectrum for an event in which only NB is contributing to the anti-Stokes signal by way of pumping, but $N B$ and $C V$ are contributing to the Stokes signal. This figure is taken from figure 1 in reference [3].

rescence at energies above the laser line. The single molecule events were then taken as those spectra where the intensity of the $-802 \mathrm{~cm}^{-1}$ mode of $\mathrm{CV}$ or the $-592 \mathrm{~cm}^{-1}$ mode of NB was much larger than the other. In addition, a lower limit was placed on the peak intensities (which depended on the mode) below which the events are discarded due to the low signal to noise ratio. This had the unforeseen result that many of the events that contained very large intensities for the $1173 \mathrm{~cm}^{-1}$ mode were ignored, as a large $1173 \mathrm{~cm}^{-1}$ anti-Stokes peak does not necessarily correspond to a large $802 \mathrm{~cm}^{-1}$ anti-Stokes peak due to the plasmon resonance. We therefore calculated the total intensity of the $1173 \mathrm{~cm}^{-1}$ and $592 \mathrm{~cm}^{-1}$ anti-Stokes peaks by integrating the intensity over the width of the modes and subtracting a linear background. The cross-sections were then measured when the $1173 \mathrm{~cm}^{-1}$ anti-Stokes intensity was much larger than the $592 \mathrm{~cm}^{-1}$ anti-Stokes intensity.

\subsubsection{The Pumping and SERS Cross-Sections}

In order to estimate the pumping cross-section, we need an accurate measurement of both the Stokes and anti-Stokes intensity. However, because we have only used the anti-Stokes signal to identify single molecule events, mixed Stokes events may be present in some cases resulting in a slight over-estimation of the 

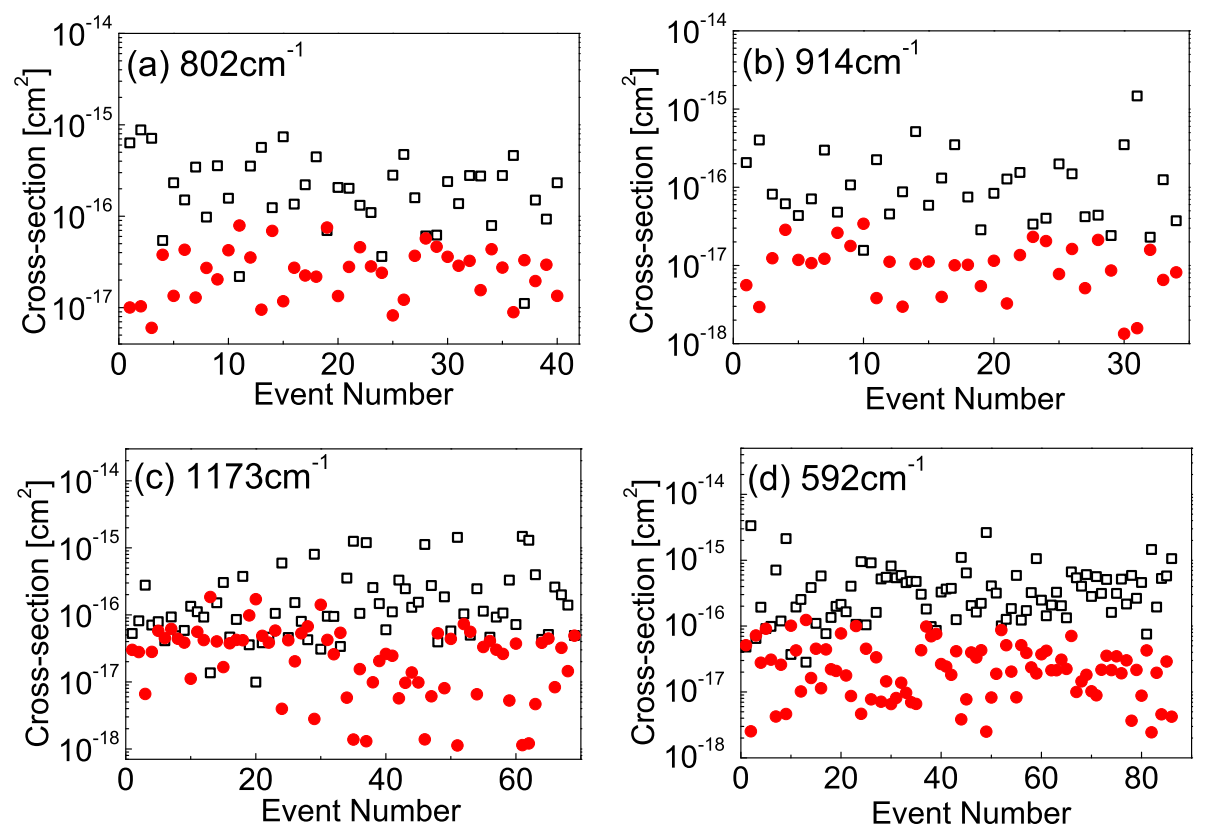

Figure 6.11: Pumping (hollow square points) and SERS cross-sections (solid circles) for the (a) $802 \mathrm{~cm}^{-1}$, (b) $914 \mathrm{~cm}^{-1}$ and (c) $1173 \mathrm{~cm}^{-1}$ modes of $C V$ and the (d) $592 \mathrm{~cm}^{-1}$ mode of $\mathrm{NB}$, as a function of event number. The cross-sections are plotted on a logarithmic scale. This figure is taken from figure 4 in reference [3].

SERS cross-section and under-estimation of the pumping cross-section.

Once the single molecule events have been identified (using the criteria explained in the previous section), the anti-Stokes and Stokes intensities of the modes listed in figure 6.8 were measured by performing an intensity integration with a linear fit to the background. At this point, it is useful to assume that the asymmetry factor is 1 for all cases. I will come back to the problem of the asymmetry factor later in this section. The pumping cross-section could then be measured from the ratio of the anti-Stokes to Stokes intensities using equation 6.4 with a $\tau$ of $1 \mathrm{ps}$. The differential SERS cross-section was estimated using the method explained in appendix $\mathrm{C}$ and converted to a total scattering cross-section as explained in appendix A.

The pumping and SERS cross-sections are plotted in figure 6.11 as a function of event number for the three peaks of CV and the single peak of NB. For all of the modes considered, there is an obvious difference between the two crosssections with the pumping cross-section typically being significantly larger than 

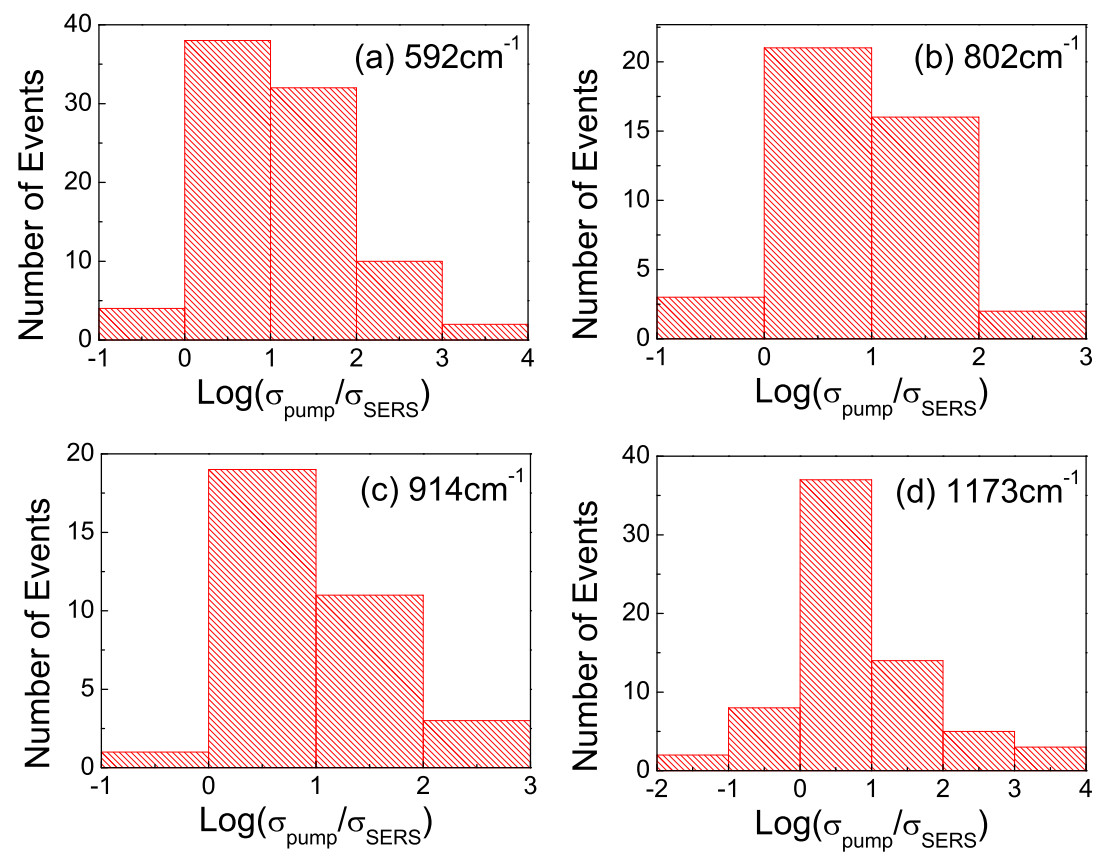

Figure 6.12: Histograms of the ratio of the pumping to SERS cross-section for the (a) $592 \mathrm{~cm}^{-1}$ mode of $\mathrm{NB}$, and the (b) $802 \mathrm{~cm}^{-1}$, (b) $914 \mathrm{~cm}^{-1}$ and (d) $1173 \mathrm{~cm}^{-1}$ modes of $C V$, with a logarithmic $x$-scale.

the SERS cross-section. The fluctuation in the cross-sections seems to be very similar as well, which is not surprising as both cross-sections depend on the local conditions experienced by the molecule. However, the fluctuations do not necessarily coincide. If the local field enhancement was the only effect causing the variation, then an event with a large SERS cross-section should also have a large pumping cross-section. This does not seem to be the case and, in fact, many of the large Stokes events have pumping cross-sections very similar to the SERS cross-section. We will come back to this problem later in this section.

To achieve a better understanding of how large the discrepancy between the two cross-sections is, it is useful to calculate the ratio between them and investigate how it varies. Figure 6.12 shows a series of histograms (with a log scale on the x-axis) of the ratio of the cross-sections for each of the measured modes. A large portion of the events have a pumping cross-section that is within one order of magnitude of the SERS cross-section. One might be inclined to justify 

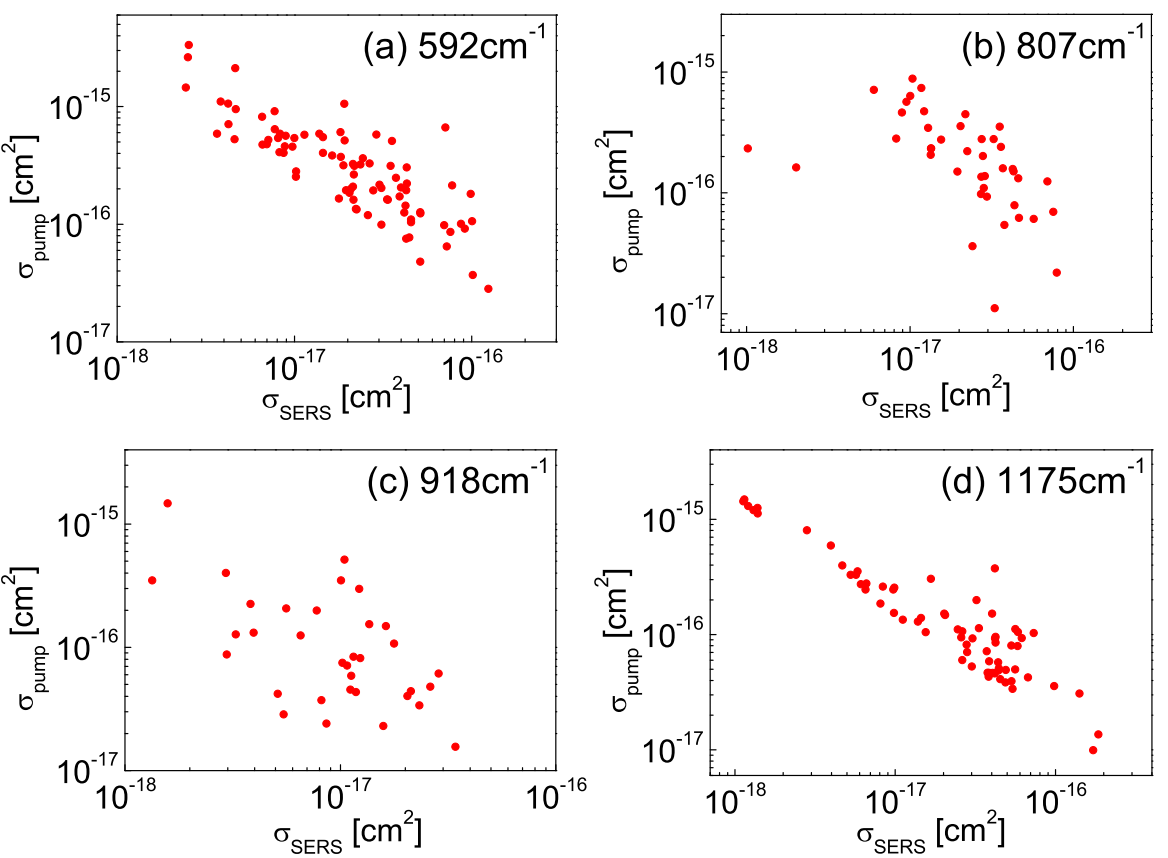

Figure 6.13: Plots of pumping cross-section as a function of SERS cross-section for the (a) $592 \mathrm{~cm}^{-1}$ mode of $\mathrm{NB}$, and the (b) $802 \mathrm{~cm}^{-1}$, (b) $914 \mathrm{~cm}^{-1}$ and (d) $1173 \mathrm{~cm}^{-1}$ modes of $C V$.

this discrepancy as a result of an under-estimation of the vibrational lifetime of the modes or perhaps an asymmetry factor that favours anti-Stokes scattering. However, there are also events with pumping cross-sections that are three or even four orders of magnitude larger than the SERS cross-section. If we try to justify this with the vibrational lifetime then a value of $100-1000 \mathrm{ps}$ is needed which is physically unreasonable. A lifetime of this length would be apparent in the line-width of the peaks, which would be closer to $\sim 0.1 \mathrm{~cm}^{-1}$ unless dephasing (see section 4.4) plays a significant role. Furthermore, modifying the vibrational lifetime will only have the effect that the average cross-sections will coincide, it will not explain the fluctuation as the lifetime will be the same for all SERS events.

Earlier in this section, I noted that even though the pumping and SERS cross-sections varied by a similar amount, they did not appear to coincide with each other. This is confirmed in figure 6.12. The pumping cross-section can be three, or even four, orders of magnitude larger than the SERS cross-section, 
whilst the individual fluctuation in each cross-section can vary by a factor of 10-100. The question then arises, how are the pumping and SERS cross-sections related? In figure $6.13 \mathrm{I}$ have plotted the cross-sections as a function of each other. One would expect, that as the SERS cross-section increases, the pumping cross-section should also increase due to the strength of the local field enhancement. This, however, does not seem to be the case. In fact, the opposite occurs, $\sigma_{\text {pump }}$ seems to decrease with an increasing $\sigma_{\text {SERS }}$. To explain this trend we must address the interpretation of the pumping cross-section and how is it affected by the plasmon resonance.

\section{Non-Radiative Effects}

In section 4.2.5, I explained that the pumping cross-section is affected by both radiative and non-radiative Stokes scattering such that:

$$
\sigma_{\text {pump }}=\frac{\left\langle\sigma_{S}^{T} d \sigma_{a S}^{R} / d \Omega\right\rangle}{\left\langle d \sigma_{a S}^{R} / d \Omega\right\rangle}
$$

For single molecule pumping, this becomes:

$$
\sigma_{\text {pump }}=\sigma_{S}^{T}=\sigma_{S}^{R}+\sigma_{S}^{N R} .
$$

Exactly which of the two Stokes cross-sections dominates the total cross-section will therefore depend on the local environment of the observed molecule. The origin of the enhancement for each cross-section is radically different, with the radiative cross-section depending only on the strength of the local field enhancement, whilst the non-radiative cross-section is modified by the distance of the molecule from the metallic surface (as explained in section 3.2). If the radiative cross-section was the dominant term, then we would expect that the measured SERS cross-section (which is the same as the radiative cross-section) would be the same as the pumping cross-section and fluctuate together (with minor differences due to the asymmetry factor and photo-bleaching). This was not the case, however, and in fact, $\sigma_{\text {pump }}$ tended to decrease as the SERS cross-section increased (see figure 6.13). If the non-radiative term dominated the total crosssection, then $\sigma_{\text {pump }}$ would only vary slightly between events as most of the molecules would be at a similar distance to the metal surface (due to the adsorption characteristics of the molecule). The downward trend in $\sigma_{\text {pump }}$ would then be a result of the restrictions in the possible asymmetry factors, as I will explain in the next section. However, at this point, a lot of this is speculation 
unless we can obtain direct measurements of the non-radiative enhancement factor and confirm that it is larger than the radiative enhancement factor. A large section of this chapter has therefore been dedicated to an investigation into the non-radiative enhancement factor (see section 6.5).

\section{The Asymmetry Factor}

In figure 6.13 we observed that the pumping and SERS cross-sections appear to be inversely related. This is partly due to the fact that we have assumed that $A=1$. In fact, what is plotted in figure 6.13 is $A \sigma_{\text {pump }}$ against $\sigma_{\text {SERS }}$. If $\sigma_{\text {pump }}$ was dominated by radiative Stokes scattering, the anti-Stokes intensity ratio would be given by:

$$
I_{a S}=\frac{\left(\sigma_{S}^{R} d \sigma_{a S}^{R} / d \Omega\right) \tau P_{L}^{2}}{\hbar \omega_{L}} .
$$

In section 3.2.1 I explained how the SERS signal receives an enhancement from the plasmon resonance at the incident photon wavelength and also at the scattered photon wavelength. Because the anti-Stokes intensity is proportional to both the anti-Stokes and Stokes radiative cross-sections, it is possible to have a plasmon resonance that favours Stokes scattering create the same anti-Stokes intensity as one that favours anti-Stokes scattering (see figure 6.14(a) and (b)). As a result, for sufficiently strong plasmon resonances, we would expect that pumping would be observable for a mode in which its anti-Stokes or Stokes peak is favoured by the plasmon resonance. However, in section 6.3 .2 we saw that it was possible to have events with extremely large single molecule Stokes signals with no evidence of vibrational pumping in the anti-Stokes signal.

If the non-radiative cross-section dominates the pumping cross-section, the anti-Stokes intensity will be given by:

$$
I_{a S}=\frac{\left(\sigma_{S}^{N R} d \sigma_{a S}^{R} / d \Omega\right) \tau P_{L}^{2}}{\hbar \omega_{L}} .
$$

In this case, the non-radiative cross-section no longer receives a local field enhancement at the scattered wavelength (see section 4.2.5) but instead experiences the non-radiative enhancement factor $M_{N R}\left(\omega_{S}\right)$. This has the effect that the anti-Stokes intensity is only dependent on the plasmon resonance at the anti-Stokes wavelength.

If the non-radiative Stokes scattering is the dominant component in the pumping cross-section, then the inverse relationship between $\sigma_{\text {pump }}$ and $\sigma_{S}^{R}$ can 
be explained purely from the asymmetry factor. Let us consider a scenario in which there is a limit to the strength of the plasmon resonance. This is not an unreasonable assumption, as there will be a limit to the size of the gap between the metallic particles (where the largest enhancements form) if a molecule is to be localized within the hot-spot. The dispersion of the plasmon resonance will vary from case to case which will result in a fluctuating asymmetry factor. The plasmon resonances that create the largest Stokes cross-section will be the ones that have a sharp resonance on the Stokes side of the spectrum (see figure 6.14(c)). As a consequence, the enhancement to the anti-Stokes signal will be relatively small and the asymmetry factor will be less than one. The pumping cross-section will therefore be underestimated when we assume $A=1$. The events with the largest anti-Stokes intensities will require a plasmon resonance that favours anti-Stokes scattering resulting in relatively low Stokes intensities (see figure 6.14(d)). The pumping cross-section in this case will therefore be overestimated. The overall effect is that the estimated pumping cross-section will tend to decrease as the Stokes intensity increases.

In addition, there may be a slight contribution due to photo-bleaching. While the pumping cross-section is independent of photo-bleaching, the SERS crosssection is not. The largest pumping events may therefore have SERS crosssections that have been under-estimated as the molecule will survive for a shorter time than the integration time.

The results of figure 6.13 can now be explained with a pumping cross-section that is dominated by non-radiative effects for all cases, and an asymmetry factor that is restricted by the SERS cross-section (as well as a slight contribution due to photo-bleaching). The smallest Stokes events, that show pumping, are likely created by relatively weak plasmon resonances with large asymmetry factors that enhance the anti-Stokes signal. Conversely, the largest Stokes events will likely have small asymmetry factors as a favourable plasmon resonance is required to reach such large intensities. Furthermore, because the non-radiative cross-section fluctuates a lot less than the radiative cross-section (explained in the next section), the asymmetry factor will be the main contribution to the variation of $\sigma_{\text {pump }}$ in figure 6.13 . 

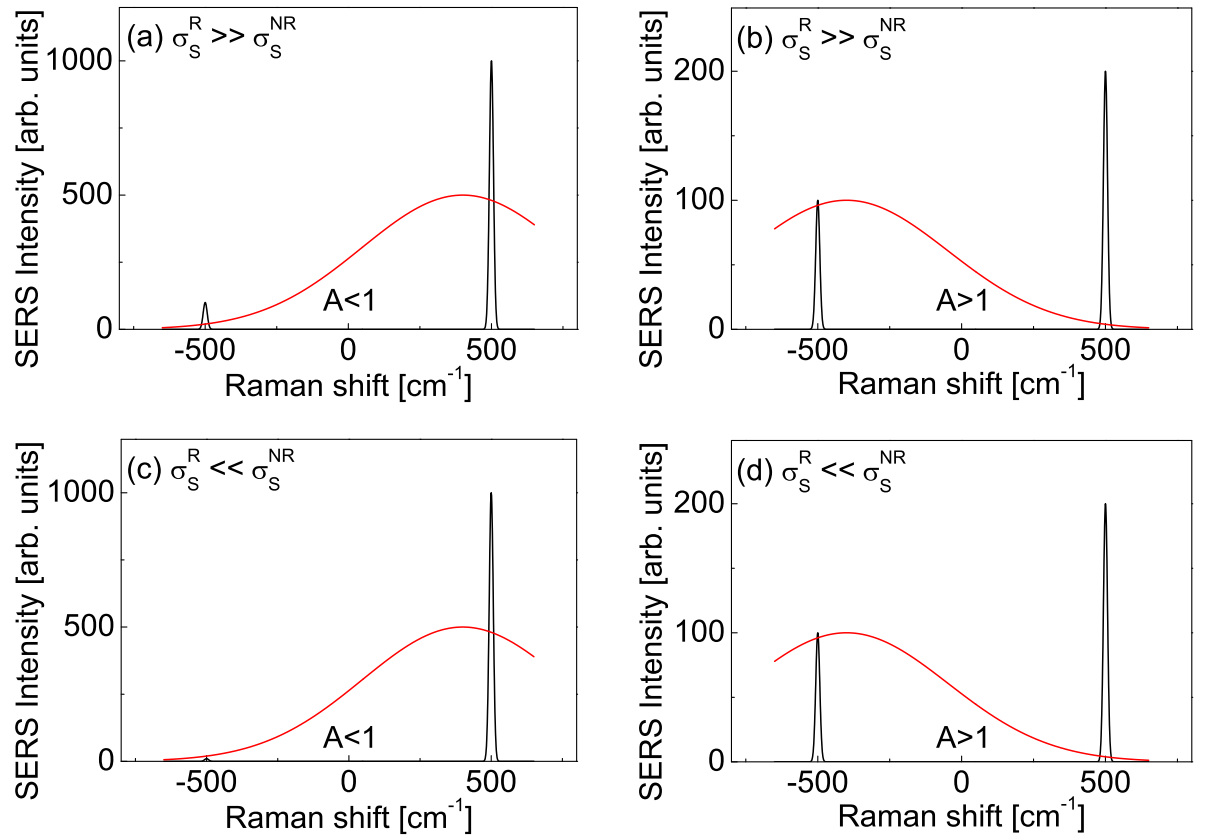

Figure 6.14: Simulated SERS spectra (black lines) for a single mode with both antiStokes and Stokes scattering under pumping conditions and different plasmon resonances (red lines). In (a) and (c), the plasmon resonance favours Stokes scattering resulting in a asymmetry factor of less than 1. In (b) and (c), the plasmon resonance favours anti-Stokes scattering resulting in an asymmetry factor larger than 1. In (a) and (b), the radiative cross-section dominates the pumping cross-section. In (c) and (d), the non-radiative cross-section dominates the pumping cross-section. 


\subsection{An Investigation into the Non-Radiative En- hancement Factor}

In the previous section I speculated that the pumping cross-section was dependent on the non-radiative cross-section, in typical single molecule experiments, and not the radiative cross-section as was previously believed. However, in order to confirm this hypothesis, we need a technique for which we can measure both the non-radiative and radiative enhancement factors simultaneously [168]. In section 3.2.1 I explained that the radiative Stokes cross-section is a modification of the bare Raman cross-section via:

$$
\sigma_{S}^{R}=M_{\mathrm{Loc}}\left(\omega_{L}\right) M_{\mathrm{Rad}}\left(\omega_{S}\right) \sigma_{\mathrm{Raman}}
$$

in terms of total scattering cross-sections. Similarly, the non-radiative Stokes cross-section is a modification of the bare Raman cross-section but with the non-radiative enhancement factor (see section 4.2.5) in the emission step:

$$
\sigma_{S}^{N R}=M_{\mathrm{Loc}}\left(\omega_{L}\right) M_{\mathrm{NR}}\left(\omega_{S}\right) \sigma_{\mathrm{Raman}}
$$

The difference being that the radiative enhancement factor, $M_{R a d}\left(\omega_{S}\right)$, depends on the local field strength at the Stokes shifted frequency, whilst the non-radiative enhancement factor, $M_{N R}\left(\omega_{S}\right)$, depends mostly on the distance of the molecule from the metal surface (see section 3.2.2). Because the only SERS processes that will be detected in the far field, and hence measured by an observer, are radiative, it is very difficult to measure the non-radiative enhancement factor. To the best of our knowledge, vibrational pumping is the first example in SERS where non-radiative effects may be directly observed. However, there is another area of research in which non-radiative effects play an important role: Surface Enhanced Fluorescence.

\subsubsection{SEF and SERS Comparisons}

In section 3.2 it was shown that the SERS and SEF enhancement factors could be given by:

$$
M_{\mathrm{SEF}}=M_{\mathrm{Loc}}\left(\omega_{L}\right) \frac{M_{\mathrm{Rad}}\left(\omega_{S}\right)}{Q^{0} M_{\mathrm{Tot}}}
$$

and 

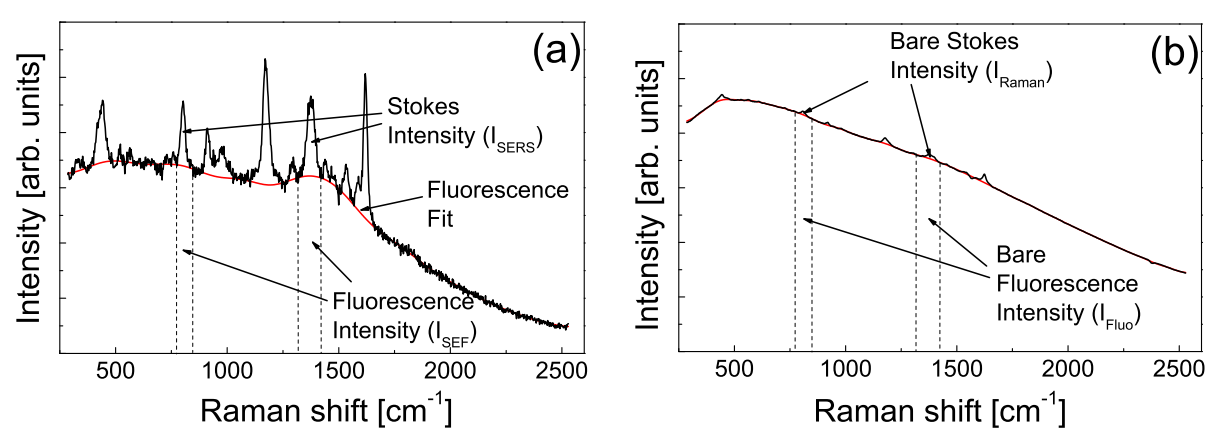

Figure 6.15: Spectra of $C V$ taken under (a) single molecule surface enhanced conditions, and (b) $100 \mu \mathrm{M}$ solution with no colloidal aggregates. Both spectra also contain a fluorescence fit (red line) performed with the wavelet based application discussed in chapter 5. The Raman intensity is shown in both cases and the fluorescence intensity is measured over the same regions. This figure is adapted from figure 1 in reference [168].

$$
M_{\mathrm{SERS}}=M_{\mathrm{Loc}}\left(\omega_{L}\right) M_{\mathrm{Rad}}\left(\omega_{S}\right) .
$$

Taking the ratio of these two equations we obtain:

$$
R=\frac{M_{\mathrm{SERS}}}{M_{\mathrm{SEF}}}=Q^{0} M_{\mathrm{Tot}}
$$

Hence, if we know the quantum yield of a molecule, we can estimate $M_{\text {Tot }}$ from the ratio of the SEF and SERS enhancement factors. In terms of signal intensities, we can rewrite equation 6.14 as:

$$
R=\frac{I_{\mathrm{SERS}}\left(\omega_{S}\right) / I_{\mathrm{Raman}}\left(\omega_{S}\right)}{I_{\mathrm{SEF}}\left(\omega_{S}\right) / I_{\text {Fluo }}\left(\omega_{S}\right)}=\frac{I_{\mathrm{SERS}}\left(\omega_{S}\right)}{I_{\mathrm{SEF}}\left(\omega_{S}\right)} \frac{I_{\mathrm{Fluo}}\left(\omega_{S}\right)}{I_{\mathrm{Raman}}\left(\omega_{S}\right)}=Q^{0} M_{\mathrm{Tot}} .
$$

Therefore, by measuring the Raman intensity of a vibrational mode, and the fluorescence intensity directly beneath it, under both surfaced-enhanced and unmodified conditions, we can estimate the value for $M_{\text {Tot }}$. An example is shown in figure 6.15 for $\mathrm{CV}$. It is important that the region over which the fluorescence intensity is measured is the same for both cases.

The surface enhanced intensities must be measured under single molecule conditions as the local field enhancement can vary substantially for different events. Furthermore, the concentration used for the unmodified events must be low enough that there is no significant interaction between the molecules. 
Equation 6.15 has the benefit that it is independent of photo-bleaching problems as $I_{\mathrm{SEF}}$ and $I_{\mathrm{SERS}}$ will increase linearly with the lifetime of the molecule. The ratio of these two intensities is therefore independent of the integration time. This is a very attractive prospect in any single molecule SERS application where the excitation intensities and local enhancements are large enough that photo-bleaching presents a significant problem [169].

\subsubsection{Experimental Method}

For this experiment, we chose to use CV as our dye, which has the following benefits:

- It is close to resonance when using a $633 \mathrm{~nm}$ excitation. This is beneficial in single molecule measurements when being near resonance allows us to regularly observe SERS events.

- $\mathrm{CV}$ has a low quantum yield. Because we are close to resonance, it is very difficult to measure unmodified Raman intensities due to the overwhelming fluorescence intensity for many common SERS probes (without utilizing more sophisticated techniques $[170,171])$. CV has the benefit that the bare fluorescence signal is relatively small, even close to resonance, due to its low quantum yield. As a result, the fluorescence intensity is small enough that accurate measurements of the Raman peaks can be made.

A liquid sample was prepared (see section 4.3.3) with CV dye at a final concentration of $1 \mathrm{nM}$, well within the single molecule regime for SERS measurements [109]. A sample consisting of $100 \mu \mathrm{M}$ of CV was also prepared so that an unmodified spectrum could be taken.

The spectra were taken using the experimental set-up described in section 6.3 but using a $\times 100$ immersion objective. To test for the effects of photo-bleaching, we used an incident power of $4.1 \mathrm{~mW}\left(1.34 \times 10^{10} \mathrm{~W} / \mathrm{m}^{2}\right.$ power density $), 434 \mu \mathrm{W}$ $\left(1.41 \times 10^{9} \mathrm{~W} / \mathrm{m}^{2}\right)$ and $35.7 \mu \mathrm{W}\left(1.16 \times 10^{8} \mathrm{~W} / \mathrm{m}^{2}\right)$ over a $0.625 \mu \mathrm{m}$ diameter laser spot. Each surface enhanced spectrum was integrated for $0.1 \mathrm{~s}$ for a total of 3000 spectra per time series. The sample was then centrifuged at 14, $500 \mathrm{rpm}$ for 10 minutes so that a sample without the colloids could be syphoned off. Spectra of this new sample were then taken and averaged to construct a background signal that could be subtracted from all of the single molecule events.

A spectrum of the $100 \mu \mathrm{M}$ sample was then taken with an integration time of $5 \mathrm{~s}$ and averaged 100 times, followed by a water spectrum under the same 
Figure 6.16: $A$ typical SERS spectrum showing the vibrational modes of $C V$. The Raman intensities were measured for the $441 \mathrm{~cm}^{-1}$, $803 \mathrm{~cm}^{-1}, \quad 1172 \mathrm{~cm}^{-1}$, $1386 \mathrm{~cm}^{-1}$ and $1621 \mathrm{~cm}^{-1}$ modes and the fluorescence intensities were measured from the area directly beneath each.

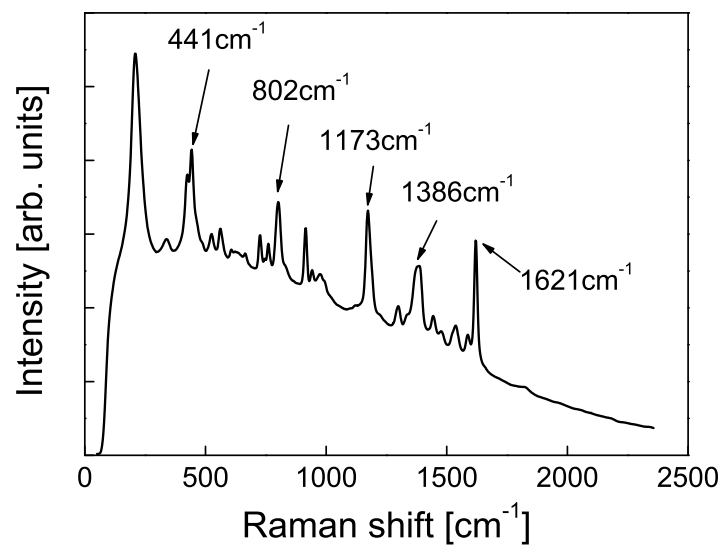

conditions so that the water background could be removed.

\subsubsection{Single Molecule Events}

A typical spectrum for $\mathrm{CV}$ is shown in figure 6.16. The peaks we chose to monitor were the $441 \mathrm{~cm}^{-1}, 803 \mathrm{~cm}^{-1}, 1172 \mathrm{~cm}^{-1}, 1386 \mathrm{~cm}^{-1}$ and $1621 \mathrm{~cm}^{-1}$ modes because they have significantly large cross-sections and can be distinguished in the unmodified spectrum.

In order to accurately measure the SERS and SEF intensities, we needed to be able to perform accurate fits to the fluorescence background for many spectra. A simple linear fit to the background under each peak could have been performed, but the low signal to noise ratio in a lot of cases, resulted in wildly varying results depending on where we set the peak region. Individually removing the background was also not possible due to the large number of events (in the thousands). The wavelet based background removal application described in chapter 5, however, is ideal for this situation as it can accurately fit fluctuating backgrounds for many spectra without any intervention from the user. The algorithm developed in chapter 5 was, therefore, somewhat serendipitous in that it seemed tailor-made for the this kind of problem.

Because we were measuring single molecule events, the SEF background could vary dramatically between spectra and contain frequency components that were relatively high (due to sharp plasmon resonances). As a result, the decomposition level could not be larger than 6 or the fit would not converge on the background for many of the events. Due to the low scaling level, an additional background region (other than one at each end of the spectrum) had 


\begin{tabular}{|c|cc|}
\hline Position $\left(\mathrm{cm}^{-1}\right)$ & From $\left(\mathrm{cm}^{-1}\right)$ & To $\left(\mathrm{cm}^{-1}\right)$ \\
\hline 441 & 373 & 502 \\
803 & 774 & 879 \\
1172 & 1094 & 1252 \\
1386 & 1317 & 1414 \\
1621 & 1567 & 1668 \\
\hline
\end{tabular}

Table 6.1: The peak location and regions over which the fluorescence and Raman intensities were integrated.

to be defined between the $441 \mathrm{~cm}^{-1}$ and $803 \mathrm{~cm}^{-1}$ modes. The other parameters defined for the fitting were the same as the typical ones listed in table 5.3. In the previous chapter, this example was used to show how accurate the algorithm was for variable backgrounds. Figure 5.19 shows four events with very different backgrounds, each fitted with the same parameters described above. In each case, the background removal application performs an extremely accurate fit without any perturbation due to the SERS peaks. Furthermore, the spectral location of the plasmon resonance does not appear to affect the accuracy of the fit.

Once the background was removed from all of the events, the peak regions (in terms of Raman shift) could then be defined (see table 6.1). The SERS intensity was calculated from the integrated intensity over the defined regions for the signal with the background removed (figure 6.17(b)). The SEF intensity was measured from the integrated intensity, over the same regions, between the background fit (figure 6.17(a)) and the background-only spectrum (the spectrum taken from the centrifuged sample). The ratio of the SERS and SEF intensity for each of the peaks and events was then calculated.

This process was then repeated for the $100 \mu \mathrm{M}$ spectrum to obtain a value for $I_{\text {Raman }}$ and $I_{\text {Fluo }}$ using the same fitting parameters used in the surfaceenhanced case, and the same peak regions defined in table 6.1. The ratio of the Raman and fluorescence intensities were calculated to be; 0.0054 for the $441 \mathrm{~cm}^{-1}$ mode, 0.0048 for the $803 \mathrm{~cm}^{-1}$ mode, 0.0081 for the $1172 \mathrm{~cm}^{-1}$ mode, 0.0104 for the $1386 \mathrm{~cm}^{-1}$ mode, and 0.0136 for the $1621 \mathrm{~cm}^{-1}$ mode. A value for $\mathrm{R}\left(=Q^{0} M_{\text {Tot }}\right)$ could then be measured for every event by taking the quotient of the SERS and SEF intensity ratio and the Raman and fluorescence intensity ratio.

Because we were calculating intensity ratios, it was important that we filtered out events that were particularly small and may cause singularities or 


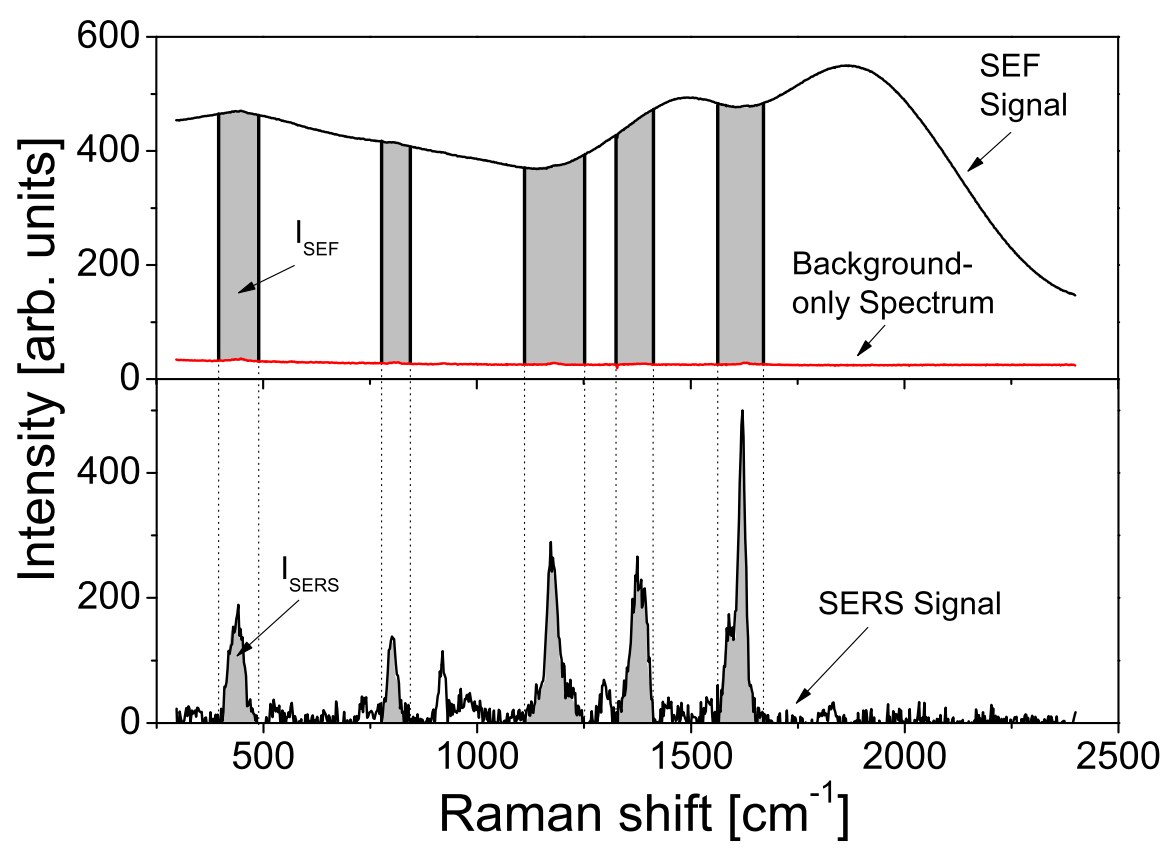

Figure 6.17: The (a) background and (b) SERS spectrum of a single molecule event. The SERS intensity is estimated from the integrated intensity of the SERS spectrum over the regions defined in table 6.1. The SEF intensity is estimated from the overall intensity between the SEF signal and the "background-only" spectrum, over the same regions. 
inaccuracies. Therefore, all intensities where the maximum SERS peak height was less than 200 counts/s were discarded. This was approximately double the noise level which had a maximum height of approximately 100 counts/s.

\subsubsection{The Quantum Yield of Crystal violet}

In order to estimate the value of $M_{\text {Tot }}$ from the value of $\mathrm{R}$, we had to measure the unmodified quantum yield, $Q^{0}$, of $\mathrm{CV}$ when dissolved in water (the solvent used for our experiments). The quantum yield is the fraction of the absorbed photons that are re-emitted as fluorescence. Hence, for an incident wavelength with a known absorption cross-section, $\sigma_{\mathrm{Abs}}\left(\omega_{L}\right)$, the quantum yield can be calculated from the ratio:

$$
Q^{0}=\frac{\sigma_{\text {Fluo }}}{\sigma_{\mathrm{Abs}}},
$$

where $\sigma_{\text {Fluo }}$ is the total fluorescence cross-section over all wavelengths. Hence, by measuring these two cross-sections we can estimate the quantum yield of the molecule.

\section{Measuring the Absorption Cross-Section}

The Beer-Lambert Law states that the absorbance and absorption cross-section are related via:

$$
A=-\log _{10}\left(\exp \left(-\sigma_{\mathrm{Abs}} l N\right)\right),
$$

where $l$ is the path length that the laser travels through the liquid sample, and $N$ is the number density of the absorber (CV in this case). Using a UV/vis spectrometer we obtained an absorbance of 0.07844 at $633 \mathrm{~nm}$ over a path length of $1 \mathrm{~cm}$ and a concentration of $10 \mu \mathrm{M}$. This corresponds to an absorption crosssection of $3 \times 10^{-17} \mathrm{~cm}^{2}$.

\section{Measuring the Total Fluorescence Cross-Section}

The fluorescence cross-section is measured the same way that we measure a single molecule SERS cross-section (see appendix C), by comparing the overall fluorescence intensity with the Raman intensity of a molecule with a known cross-section (2B2MP in this case) measured under the same experimental condition. 


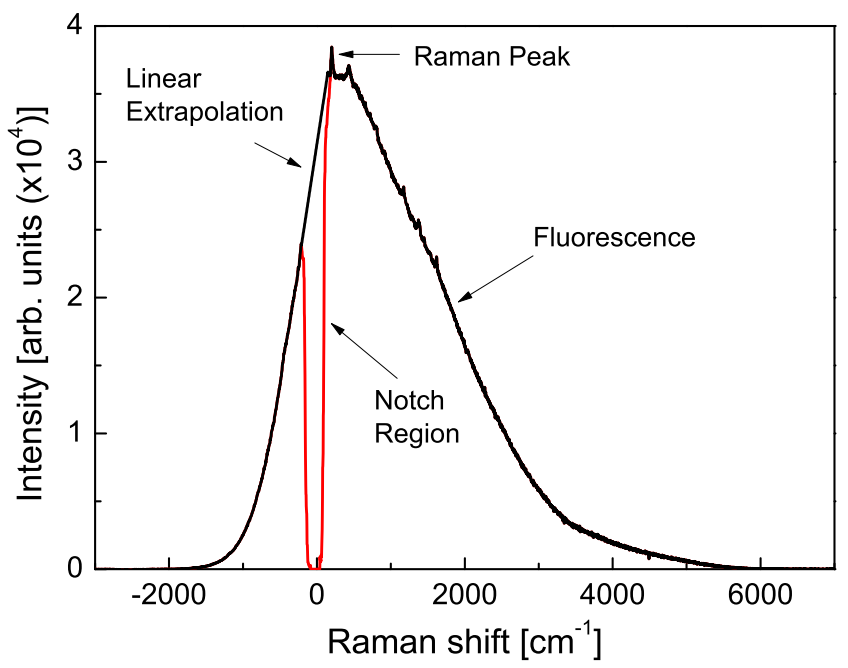

Figure 6.18: The fluorescence spectrum of a $10 \mu M$ sample of $C V$ taken over a range of $-3000 \mathrm{~cm}^{-1}$ to $7000 \mathrm{~cm}^{-1}$ for a $633 \mathrm{~nm}$ excitation. A linear extrapolation was performed on either side of the notch filter region so that we could estimate the intensity there.

Using an excitation wavelength of $633 \mathrm{~nm}$, we took a spectrum of a sample of $10 \mu \mathrm{M} \mathrm{CV}$ ranging from $-3000 \mathrm{~cm}^{-1}$ to $7000 \mathrm{~cm}^{-1}$ (large enough to obtain the full fluorescence spectrum) with an integration time of $25 \mathrm{~s}$. We also took a water spectrum under the same conditions and subtracted it from the fluorescence signal. Due to the notch filter, there was a region around the laser frequency where the fluorescence was "cut-out". We therefore, performed a linear extrapolation from either side of the notch filter region as shown in figure 6.18, and measured the total integrated intensity over the region from $-2000 \mathrm{~cm}^{-1}$ to $6000 \mathrm{~cm}^{-1}$. The intensity was then converted into a differential scattering cross-section (see appendix C) and then to a total fluorescence cross-section of $1.588 \times 10^{-21} \mathrm{~cm}^{2}$ (see appendix A). Using equation 6.16 and the fluorescence and absorption cross-sections, we estimated the unmodified quantum yield to be $Q^{0} \approx 5.3 \times 10^{-5}$, which is in close agreement with the value $Q^{0}=5.6 \times 10^{-5}$ obtained in reference [172].

Due to the extremely low quantum yield of CV, our assumption that $Q^{0}>>$ $1 / M_{\text {Tot }}$ may no longer be valid for the derivation of the modified quantum yield in equation 3.14. However, for a molecule with an extremely small quantum yield, we can rewrite equation 2.17 as: 


$$
Q^{0}=\frac{\Gamma_{\mathrm{Rad}}}{\Gamma_{\mathrm{NR}}^{0}},
$$

which results in a modified quantum yield of:

$$
Q^{M}=\frac{M_{\mathrm{Rad}} \Gamma_{\mathrm{Rad}}}{M_{\mathrm{Tot}} \Gamma_{\mathrm{Rad}}+\Gamma_{\mathrm{Rad}} / Q^{0}}=\frac{M_{\mathrm{Rad}}}{M_{\mathrm{Tot}}+1 / Q^{0}},
$$

and a fluorescence enhancement factor of:

$$
M_{\text {Fluo }}=M_{\text {Loc }}\left(\omega_{L}\right) \frac{M_{\text {Rad }}\left(\omega_{S}\right)}{Q^{0} M_{\text {Tot }}+1} .
$$

The ratio of the SEF and SERS enhancement factor will therefore be:

$$
R=Q^{0} M_{\text {Tot }}+1 .
$$

Comparing this with equation 6.14 we can see that $M_{\text {Tot }}$ will be overestimated by a factor $1 / Q^{0}$ or $1.9 \times 10^{4}$ for $\mathrm{CV}$.

\subsubsection{A Comparison between $M_{\text {Tot }}$ and $M_{\text {Rad }}$}

Using the quantum yield measured in the previous section, we can calculate a value of $M_{\text {Tot }}$ for each peak and event. Furthermore, we can measure a value for the SERS enhancement factor, $M_{\mathrm{SERS}}=M_{\mathrm{Loc}}\left(\omega_{L}\right) M_{\mathrm{Rad}}\left(\omega_{S}\right)$, from the ratio of the SERS and Raman intensities (after each intensity has been converted into units of counts per second per molecule), under the same excitation power.

Because $M_{\text {Tot }}$ is the total enhancement of all decay processes from the first electronic state, the value should be independent of the peak used to measure it. Table 6.2 shows the average $M_{\text {Tot }}$ measured for each peak at each incident power. As expected, $M_{\text {Tot }}$ does not vary significantly with most of the values being within $20 \%$ of the average, which is not unexpected for the experimental inaccuracies inherent in these measurements. There could be a slight contribution due to surface selection rules $[9,173,174]$ affecting the SERS intensities for modes with different symmetries. However, under resonant conditions, the Raman mode tensors for all of the vibrational modes are aligned. There could also be a slight error due to the fluorescence fitting process. From this point, the quoted values for $M_{\text {Tot }}$ will be the average over the five measured peaks.

Table 6.2 also shows that, there is no noticeable effect on $M_{\text {Tot }}$ due to photobleaching as it is independent of the incident power. This is not surprising as we are looking at ratios in intensities which are linearly dependent on the lifetime 


\begin{tabular}{|c|c|c|c|c|c|c|}
\hline \multirow[t]{2}{*}{ Power } & \multicolumn{5}{|c|}{$\left\langle M_{\text {Tot }}\right\rangle\left(\times 10^{5}\right)$} & \multirow{2}{*}{$\left\langle M_{\text {Tot }}^{\text {Ave }}\right\rangle\left(\times 10^{5}\right)$} \\
\hline & 441 & 803 & 1172 & 1386 & 1621 & \\
\hline $4.10 \mathrm{~mW}$ & 4.96 & 4.36 & 3.80 & 4.06 & 3.91 & 4.21 \\
\hline $434 \mu \mathrm{W}$ & 5.22 & 4.78 & 3.84 & 4.23 & 4.08 & 4.43 \\
\hline $35.7 \mu \mathrm{W}$ & 5.66 & 4.75 & 3.79 & 3.85 & 3.74 & 4.36 \\
\hline
\end{tabular}

Table 6.2: The average $M_{\text {Tot }}$ for each of the peaks measured and powers of $4.1 \mathrm{~mW}$, $434 \mu \mathrm{W}$ and $35.7 \mu \mathrm{W}$. Also included is the average over all peaks.

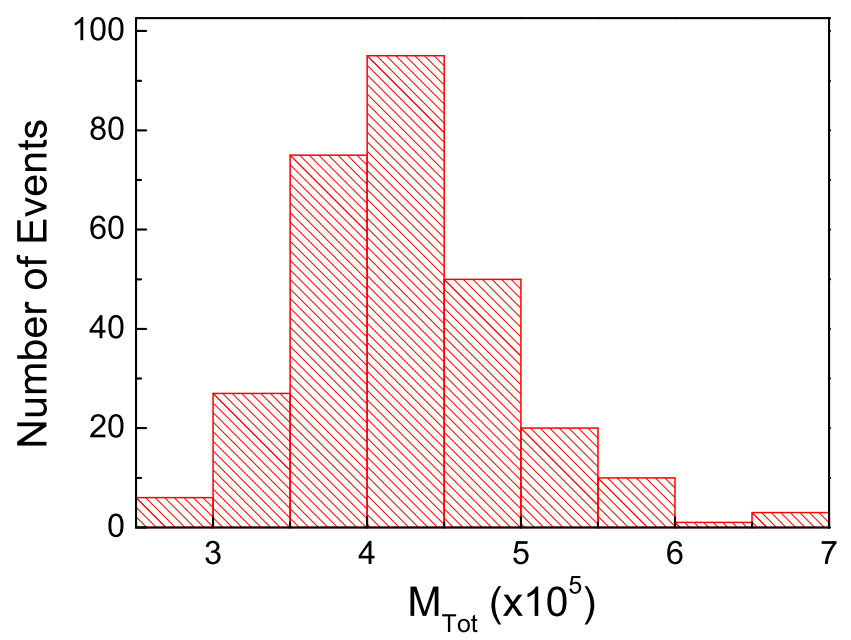

Figure 6.19: A histogram of the peak average total enhancement factor for all events. The average $M_{\text {Tot }}$ is $4.25 \times 10^{5}$ and the standard deviation is $0.69 \times 10^{5}$. This figure is taken from figure 2 in reference [168]

of the molecule.

A histogram showing how $M_{\text {Tot }}$ varies over all events is shown in figure 6.19. The average is $4.25 \times 10^{5}$ with a standard deviation of $0.69 \times 10^{5}$. Consequently, there is very little fluctuation in the value of $M_{\text {Tot }}$. Because $M_{\text {Rad }}$ is dependent on the strength of the plasmon resonance (which will vary with event), these results suggest that $M_{\text {Tot }}$ is being dominated by non-radiative effects which are approximately constant (for molecules similarly adsorbed on the colloids).

In section 6.5.4 I showed that the measured value of $M_{\text {Tot }}$ would be overestimated by a factor of approximately $2 \times 10^{4}$. The real $M_{\text {Tot }}$ will therefore have an average that is closer to $4 \times 10^{5}$. However, this is only a difference of $5 \%$ which is insignificant compared to the overall variation in $M_{\text {Tot }}$.

To within an order of magnitude, we can assume that $M_{\mathrm{SERS}} \approx M_{\mathrm{Rad}}^{2}$ since $M_{\mathrm{Loc}} \approx M_{\mathrm{Rad}}$ to within an order of magnitude (see section 3.2.1). We can 


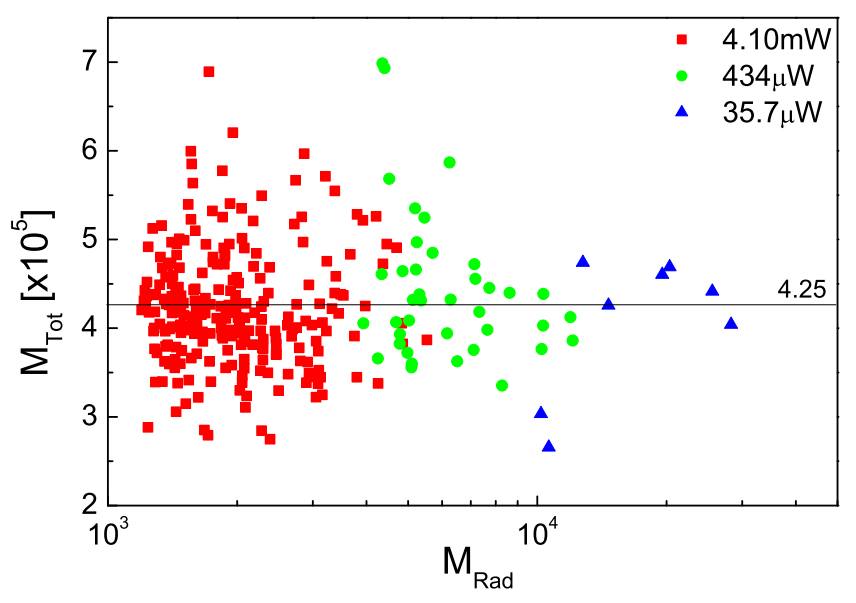

Figure 6.20: Plot of $M_{\text {Tot }}$ as a function of $M_{\mathrm{Rad}}$ for an incident laser power of $4.1 \mathrm{~mW}, 434 \mu \mathrm{W}$ and $35.7 \mu \mathrm{W}$, for all of the single molecule events.

therefore convert our SERS enhancement factors, which we estimated from the ratio of the SERS and Raman intensities, into $M_{\text {Rad }}$ by taking the square root. Figure 6.20 shows $M_{\text {Tot }}$ plotted as a function of $M_{\text {Rad }}$ for all events and incident powers. The fluctuation in $M_{\text {Tot }}$ is independent of both the power and $M_{\text {Rad }}$. This is not surprising considering the results in table 6.2 and figure 6.19.

As the incident power is decreased, the average lifetime of the molecules will increase. Consequently, the molecules in the largest hot-spots will survive for a longer period of time (approaching the integration time of the spectrometer), and a larger $M_{\text {SERS }}$ will be measured. It is important to note at this point that the measured value of $M_{\text {SERS }}$ is not quite the actual SERS enhancement experienced by the molecule, but one that is modified like so:

$$
M_{\mathrm{SERS}}^{\text {measured }}=M_{\mathrm{SERS}}^{\text {actual }} \times \frac{\tau}{T},
$$

where $\tau$ is the time that the molecule survives in the hot-spot, and $T$ is the integration time. This will cause a slight under-estimation of the measured value for $M_{\text {Rad }}$, but at the lowest power, we do not believe that the discrepancy between the actual and measured values are more than a factor of two and not enough to make the total and radiative enhancement factors comparable.

The events with the largest radiative enhancement factors are still more than an order of magnitude smaller than the average $M_{\text {Tot }}$. Hence, non-radiative effects must dominate for all of the measured cases and $M_{\text {Tot }} \approx M_{\mathrm{NR}}$. The 
non-radiative enhancement factor must therefore be $\sim 4 \times 10^{5}$, for this sample.

From time-resolved measurements, we know that CV has a lifetime in the first electronic state of $\sim 10 \mathrm{~ns}$ in solvents with a low viscosity (like water) [98]. Under the conditions used in this experiment, the lifetime will be modified by a factor of $1 / M_{\text {Tot }}$, resulting in a molecule that will decay within $\sim 20 \mathrm{fs}$ of absorbing an incident photon. This method has therefore provided us with an insight into the ultra-fast processes on the metal surfaces, which would otherwise be inaccessible with more conventional approaches.

It is also possible to obtain an estimate of the distance between the molecule and the metal surface with equation 3.11. Using the dielectric function of $\mathrm{Ag}$ (see section 3.1.3) and the dielectric constant of water, we found that the distance must be around $2 \AA$. At this distance, however, the approximations used to derive equation 3.11 may become unrealistic and the actual distance may be slightly larger due to non-local effects [76]. A more accurate estimate of $6-7 \AA ̊$ A was made using the non-local correction predicted by the model in reference [175]. This value is more in accord with expectations.

\subsubsection{A Return to the Single Molecule Pumping Cross- Section}

In this section, we performed measurements that proved that the non-radiative enhancement factor was significantly larger than the radiative enhancement factor. The pumping cross-section measured in section 6.4 must therefore be the same as the non-radiative cross-section defined in equation 6.11 . This is not surprising considering the anomalous relationship between the pumping and SERS cross-sections observed in section 6.4.3. There will be a slight modification to $M_{\mathrm{NR}}$ since we performed the measurements on a dry sample in the pumping experiment, but it will not be significant, and non-radiative effects will still dominate the total enhancement factor.

In order to use vibrational pumping for the measurement of the radiative SERS cross-section, a local enhancement of the order of $\sim 10^{6}$ is required. This corresponds to a SERS enhancement factor of $\sim 10^{12}$ which, to the best of my knowledge, has never been observed. 


\subsection{Conclusion}

Using the BiASERS technique, we have shown that, for resonant molecules with sufficiently large cross-sections, it is possible to observe the effects of vibrational pumping at the single molecule level. We have also shown that there is a discrepancy between the pumping and SERS cross-sections that cannot be explained purely from the asymmetry factor or the vibrational lifetime.

We performed a statistical analysis on many single molecule pumping events and plotted the pumping cross-section as a function of the radiative SERS crosssection. It was found that the cross-sections were approximately inversely proportional to each other. We hypothesised that there were two main effects that were causing this:

- The pumping cross-section being dominated by the non-radiative enhancement factor. As a result, the pumping cross-section would be relatively constant and independent of the radiative enhancement factor at the Stokes scattering wavelength. This, however, does not explain the negative slope.

- The restriction on the asymmetry factor. At small SERS cross-sections, we need a large asymmetry factor that favours anti-Stokes scattering in order to observe the peak above the noise. The resulting pumping crosssection will therefore be overestimated when assuming $A=1$. For large SERS cross-sections, a plasmon resonance that favours Stokes scattering (small asymmetry factor) is required to reach such large intensities. The pumping cross-section will therefore be underestimated for these events. There may also have been a slight contribution due to photo-bleaching.

The overall effect is to create an inverse relationship between the pumping and SERS cross-section.

To confirm that the non-radiative enhancement factor was larger than the radiative enhancement factor, we performed single molecules observations and measured both SERS and SEF simultaneously. By comparing their relative intensities, we were able to estimate the total enhancement factor and the radiative enhancement factor. We found that the total enhancement factor was approximately constant for all events and was always more than an order of magnitude larger than the radiative enhancement factor. Therefore, the non-radiative enhancement factor must be the dominant term in the total enhancement factor. 
For the last 10 years, the problem of large SERS cross-sections being predicted with vibrational pumping has puzzled many researchers. Observations of single molecule vibrational pumping shed some light on the problem by proving that the SERS and pumping cross-section are, in fact, not the same. But it was not until measurements of the non-radiative enhancement factor were performed, that the discrepancy could be explained by the non-radiative Stokes cross-section being significantly larger than the radiative Stokes cross-section for many SERS events. As a result, the pumping cross-section, which depends on the total enhancement factor (radiative + non-radiative), will typically be much larger than the SERS cross-section, which is only dependent on the radiative enhancement factor. Both can differ by factors of $10^{2}$ or $10^{3}$ under typical SERS conditions. There may also be an additional contribution due to fluorescence pumping. 


\section{Chapter 7}

\section{Conclusions}

This chapter will summarise the main results of chapters 4-6. Furthermore, possibilities for future research that could strengthen our understanding of the effects described in this thesis will be discussed.

\section{Chapter 4: Vibrational Pumping}

In chapter 4 I investigated two aspects of vibrational pumping: the accurate estimate of vibrational lifetimes, and an application of vibrational pumping using two lasers for observing the dispersion in the plasmon resonance distribution.

By measuring the temperature dependence of the peak width or peak positions, it was possible to estimate the average frequency of the vibrational modes that the observed state was coupled to. If the average frequency was less than the frequency of the mode under question, we assumed that the relaxation lifetime was shorter than the dephasing lifetime and the main contribution to the peak width. The peak width could then be used to estimate the pumping crosssection. It was observed that the high energy modes were typically coupled to modes of less energy. Furthermore, the low energy modes were often coupled to vibrations of larger frequency which meant that their peak widths were dominated by dephasing. Medium frequency vibrations could be either dephasing or population relaxation dominated depending on the case. However, due to the complicated interaction between vibrational modes, it is not possible to assume that all high energy states are coupled to vibrations of less energy.

Two-laser pumping was a novel technique that was developed for the mea- 
surement of the dispersion of the plasmon resonance distribution. Localised surface plasmons will create large electric fields in the region between the metallic nano-particles called hot-spots (as discussed in chapter 3). As the excitation frequency is varied, the location of the largest hot-spots will change. As a consequence, the molecules that are observed at one wavelength may be different to molecules observed at another wavelength. Two-laser pumping allows us to observe the ability of one laser to couple to molecules that have been excited through vibrational pumping by a second laser of a different frequency. In essence, the results provide information on how the distribution of hot-spots overlap between the two excitation wavelengths. There were several complexities and limitations associated with this technique however:

- a cryostat is required to reach vibrational pumping temperatures along with a beam splitter to combine the two laser beams;

- to obtain a detailed map of the dispersion in the plasmon resonance distribution, a large number of pumping frequencies are needed;

- the pumping laser must have a lower frequency than the probe laser to ensure that the signal is not drowned out by fluorescence. This means that only half the resonance can be mapped.

Nevertheless, two-laser pumping does have the advantage that the response of the system does not have to be calibrated, as the scattering wavelength remains constant and only the population excitation wavelength varies. Furthermore, it is the only technique, that we know of, where the population created by one laser can be observed by a second one using a mechanism that does not involve the thermal population. Until further developments are done though, it will remain a "curiosity" within the broader umbrella of SERS.

\section{Chapter 5: Wavelet Transforms and Background Removal}

In chapter $5 \mathrm{I}$ introduced the concept of wavelet transforms and how they can be effectively utilised for background removal. The advantage of wavelet transforms over techniques such as Fourier transforms, is the fact that they are well suited for low frequency signal features. I proposed an algorithm that iteratively performs discrete wavelet transforms. After each iteration, the original 
signal was modified depending on the approximation spectrum obtained with the discrete wavelet transform. The iterative algorithm can be used to separate the background from signals in which there is an overlap in the frequency range of the signal peaks and the background contributions. This algorithm had the advantage that it could be applied to many signal events without modifying the parameters of the background fit.

Using the iterative algorithm, an application (the COBRA) was built for the complete de-noising and background removal of large numbers of spectra. While the de-noising could have been carried out using wavelet transforms, we chose to use Fourier transforms with a low pass filter as it is much more flexible and quicker than discrete wavelet transforms for this specific purpose. Furthermore, unlike the signal background, noise is better represented by a basis of trigonometric functions, rather than a wavelet.

To analyse the effectiveness of the application, several SERS examples were investigated: a simulated spectrum; a series of SERS spectra in which the background varied in intensity but not frequency; and a series of single molecule SERS events in which the background varied in both frequency and overall intensity. In each case, the iterative algorithm performed significantly better than the basic discrete wavelet transform fits that are often used in the literature. In addition, the most effective choice of decomposition level depended on the distribution in the signal peak sizes and their proximity to each other. The effect of defining background regions was also investigated and found to be very beneficial in obtaining accurate background fits, especially when the background frequency was relatively large.

This algorithm turned out to be crucial for the analysis of non-radiative effects on single molecules.

\section{Chapter 6: Vibrational Pumping of Single Molecu- les and Non-Radiative Processes}

Finally, chapter 6 looked at the results of single molecule vibrational pumping measurements and the interpretation of the pumping cross-section. Using the BiASERS technique, vibrational pumping of single resonant molecules were observed. In addition, both the pumping and SERS cross-section were estimated for each pumping event, and it was found that the pumping cross-section was typically much larger than the SERS cross-section. Furthermore, the discrep- 
ancy was so large, for some cases, that it could not be justified purely by the dispersion in the plasmon resonance and the vibrational lifetime. It was also observed that the pumping cross-section decreased as the SERS cross-section increased. A hypothesis was proposed that this effect was due to a combination of non-radiative Stokes processes dominating the population excitation, along with the plasmon resonance favouring anti-Stokes or Stokes scattering to different degrees.

By measuring the contribution to the overall signal from fluorescence and Raman scattering, for single molecule events, it was possible to estimate the total and radiative enhancement factors. While the radiative enhancement factor fluctuated due to the local environment of the molecules observed, the total enhancement factor remained approximately constant. Furthermore, the total enhancement factor was always more than an order of magnitude larger than the radiative enhancement factor. The non-radiative enhancement factor must therefore be larger than the radiative enhancement factor and vary only slightly between molecules. This is not surprising as the non-radiative enhancement factor is dependent on the distance between the molecule and the metal surface, and not on the local field strength. For molecules adsorbed onto the surface of metallic nano-particles, the molecule to metal spacing should vary very little.

Finally we are able to make some conclusions about the interpretation of the pumping cross-section. The original reports of vibrational pumping always assumed that the pumping cross-section was the same as the SERS cross-section. However, the measurements performed in this chapter showed that this is not the case. While the SERS cross-section is only effected by the radiative enhancement factor, the pumping cross-section is determined by the total enhancement factor which we can confidently state is dominated by non-radiative processes for molecules directly adsorbed onto a metal surface. Consequently, the pumping cross-section will always be larger than the SERS cross-section unless extremely favourable circumstances are present; i.e. the local field is extremely large (close to the theoretical limit), and the surface plasmon resonance favours Stokes scattering.

\section{Possibilities for Future Research}

While most of the original motivations for this thesis have been addressed, there are several avenues that could be investigated in the future that will add to our 
understanding of the physics involved in this research. These include:

- Obtaining detailed information on the coupling between vibrational modes for common SERS analytes. This will require high resolution temperature dependent measurements of each of the main vibrational modes in order to detect small changes in the peak width and position. Consequently, the measurements can require a significant time commitment.

- Obtain detailed mappings of the dispersion in the plasmon resonance distribution using two-laser pumping. This will require a large number of pumping laser lines. A tunable laser is therefore very appealing for these kinds of experiments. The accuracy of the technique could also be assessed using substrates that have electromagnetic properties that are well understood, Klarite [176] for example.

- There are several modifications that could be made to the COBRA application that would make it more effective. For example: an option to restrict the region in which the background fit is made, an option to use wavelet transforms for the de-noising instead of Fourier transforms, and a more effective mechanism for searching through spectra. Furthermore, for our purposes it may be convenient to have a peak fitter for the vibrational modes once the background has been removed.

- Ideally we would like to be able to measure the pumping and SERS crosssection at the same time that we measure the total and radiative enhancement factors. This will allow us to determine how accurately we can measure the pumping cross-section from the total enhancement factor and how much of a role the asymmetry factor plays. However, there are several difficulties associated with this kind of experiment. Firstly, a dry sample needs to be used for the pumping measurements. This is a problem because bare Raman scattering and fluorescence is measured at room temperature with a liquid sample. The inter-molecule interactions will, therefore, be different for the dry and liquid samples. Furthermore, the quantum yield is modified by temperature. This could be resolved if the bare measurements were made with dry samples and low temperatures, but large numbers of scattering molecules are required to observe the Raman signal and this is not possible with a dry sample.

- It would be interesting to measure the contribution to the anti-Stokes intensity from fluorescence pumping. A possible way of doing this would be 
with two-laser pumping in which one laser is tuned to the resonance of the molecule and the other is set at a wavelength where the molecule is not resonant. Two-laser pumping is then performed in two ways: using the resonant excitation as the pump, and using the non-resonant excitation as the pump. If there is no fluorescence pumping, the pump power dependence of the anti-Stokes intensity (relative to the probe) will be the same for both cases. However, if there is fluorescence pumping, using the resonant excitation as a pump will result in a larger slope in the anti-Stokes dependence to the pump power. There is one problem associated with this experiment: in one of the two cases, the probe excitation is going to be at a shorter frequency than the pump. Fluorescence due to the pump may therefore drown out the anti-Stokes signal of the probe. Nevertheless, this effect may be minimised by having a resonant excitation at a lower frequency than the non-resonant excitation. The amount of fluorescence at the non-resonant excitation will therefore be relatively small. 


\section{Appendix A}

\section{Cross-Sections}

The term cross-section is often used in nuclear and particle physics and is related to the probability that two particles will interact. Classically we can express the cross-section as a hypothetical area in which $100 \%$ of the incident particles interact with the target particle (see figure A.1). However, for most cases, this area is much smaller than the area of the actual particle.

For scattering phenomena (like Raman and fluorescence) we often discuss the differential cross-section which corresponds to the probability of observing a scattered particle within a certain solid angle. In terms of the incident power density, $S_{\text {Inc }}$ (in units $\mathrm{W} / \mathrm{m}^{2}$ ) and the differential scattered power, $d P_{\text {Sca }}(\Omega) / d \Omega$, (in units $\mathrm{W} /$ srad $)$, the differential cross-section is given by:

$$
\frac{d \sigma_{\mathrm{Sca}}(\Omega)}{d \Omega}=\left[\frac{d P_{\mathrm{Sca}}(\Omega)}{d \Omega}\right] / S_{\text {Inc }} .
$$

which is in units of $\left[\mathrm{m}^{2} / \mathrm{srad}\right]$. The quoted values for the differential cross-section will often be orientationally averaged. The overall scattering cross-section, for all scattering angles, will be given by:

$$
\sigma_{\mathrm{Sca}}=\int \frac{d \sigma_{\mathrm{Sca}}(\Omega}{d \Omega} d \Omega .
$$

In the case of Raman scattering, it can be shown that the total Raman crosssection is related to the orientationally averaged differential cross-section via [177]:

$$
\sigma_{\mathrm{Sca}}=\frac{8 \pi}{3} \frac{1+2 \rho}{1+\rho} \frac{d \sigma_{\mathrm{Sca}}}{d \Omega},
$$

where $\rho$ is the depolarization ratio. This also holds under SERS conditions. 


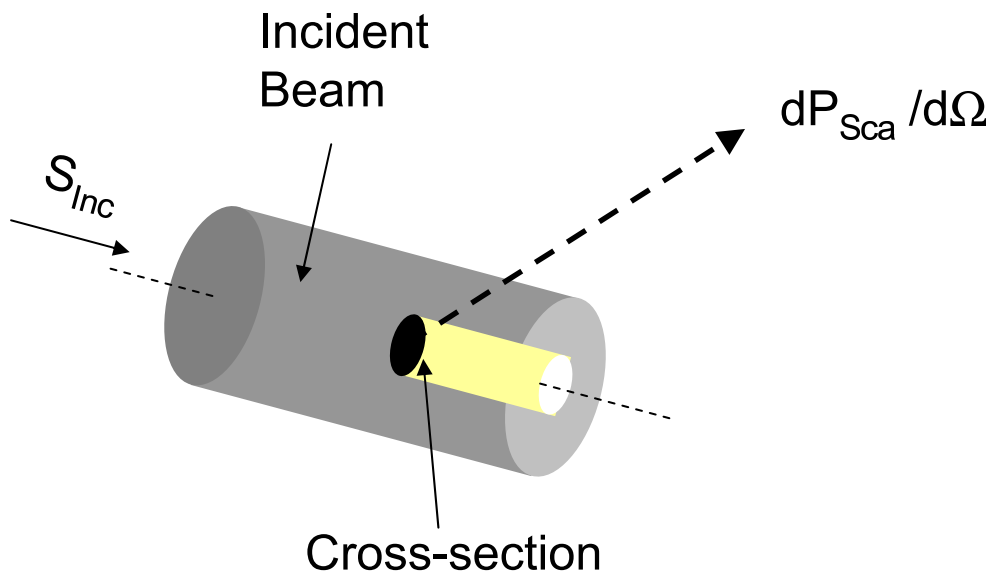

Figure A.1: A schematic of the classical interpretation of the cross-section. Included is the incident power density, $S_{\text {Inc }}$, and the differential scattered power, $d P_{\mathrm{Sca}} / d \Omega$, which is only valid for scattering interactions.

Other processes, such as absorption, do not result in a scattered particle and hence we cannot define a differential cross-section. In these cases we are only concerned with the total cross-section, which is the ratio of the energy per unit time absorbed from the incident beam, $P_{\mathrm{Abs}}$, and the incident power density:

$$
\sigma=\frac{P_{\mathrm{Abs}}}{S_{\mathrm{Inc}}}
$$




\section{Appendix B}

\section{Measuring the Scattering Volume of an Objective}

In many experiments, it is important to know the volume of the laser beam that we are collecting the scattered light from (see figure B.1). If we consider a single scattering particle at the focus of the laser, the intensity of scattered light will vary with a Gaussian distribution (see figure B.2) as the particle is moved in the plane perpendicular to the incident light, and with a Lorentzian in the direction parallel to the incident light. For a laser that is focused along the z-direction, the scattering region is equivalent to a cylinder with a constant intensity at all positions. The cylinder will have an effective width, $w_{\mathrm{Eff}}$, which will correspond to the full width at half maximum (FWHM) of the Gaussian within the $\mathrm{x}-\mathrm{y}$ plane. Furthermore, the cylinder will have an effective height, $h_{\text {Eff }}$, which will correspond to the width of the rectangle in figure B.3. The scattering volume will therefore be given by:

$$
V_{\mathrm{Sca}}=\frac{\pi w_{\mathrm{Eff}}^{2}}{4} \times h_{\mathrm{Eff}}
$$

To determine the effective width of the beam, the laser is focused onto a sample with a stable Raman signal, often silicon. The pin-hole width (aperture size) is then reduced and the change in the intensity of a Raman peak is monitored. For a square pin-hole aperture of side length $2 L$, the total scattered intensity is given by [161]: 


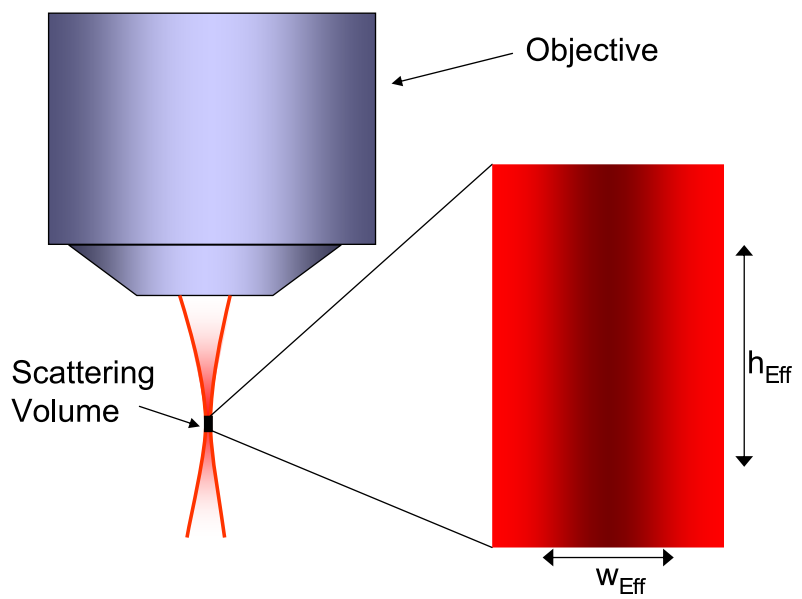

Figure B.1: A close-up of the scattering volume of the objective.

$$
\begin{aligned}
I(L)= & \frac{I_{\mathrm{Max}}}{4}\left[\operatorname{erf}\left(\frac{\sqrt{2}}{W_{0}}\left(x_{c}+L\right)\right)-\operatorname{erf}\left(\frac{\sqrt{2}}{W_{0}}\left(x_{c}-L\right)\right)\right] \\
& \times\left[\operatorname{erf}\left(\frac{\sqrt{2}}{W_{0}}\left(y_{c}+L\right)\right)-\operatorname{erf}\left(\frac{\sqrt{2}}{W_{0}}\left(y_{c}-L\right)\right)\right],
\end{aligned}
$$

where, $I_{\mathrm{Max}}$ is the intensity at the centre of the beam, $\left(x_{c}, y_{c}\right)$ are the coordinates of the centre of the beam, $W_{0}$ is the FWHM of the Gaussian beam, and $\operatorname{erf}(x)$ is the error function defined by:

$$
\operatorname{erf}(x)=\frac{2}{\sqrt{\pi}} \int_{0}^{x} e^{-t^{2}} d t .
$$

By fitting the function in equation B.2 to the variation in the Raman intensity with aperture size, we can estimate the FWHM of the Gaussian, $W_{0}$. The effective width of the beam, however, is magnified by the factor, $X$, which depends on the objective and collecting optics, so that $w_{\mathrm{Eff}}=W_{0} / X$. It is also possible to estimate the effective width by scanning the laser spot over the edge of a finely cut substrate (edge profiling). However, this is only accurate for objectives with small magnifications.

The effective height can be measured in a very similar way. The objective is moved closer to and further away from the sample and the Raman intensity is monitored. The variation in the Raman intensity will follow a response function 


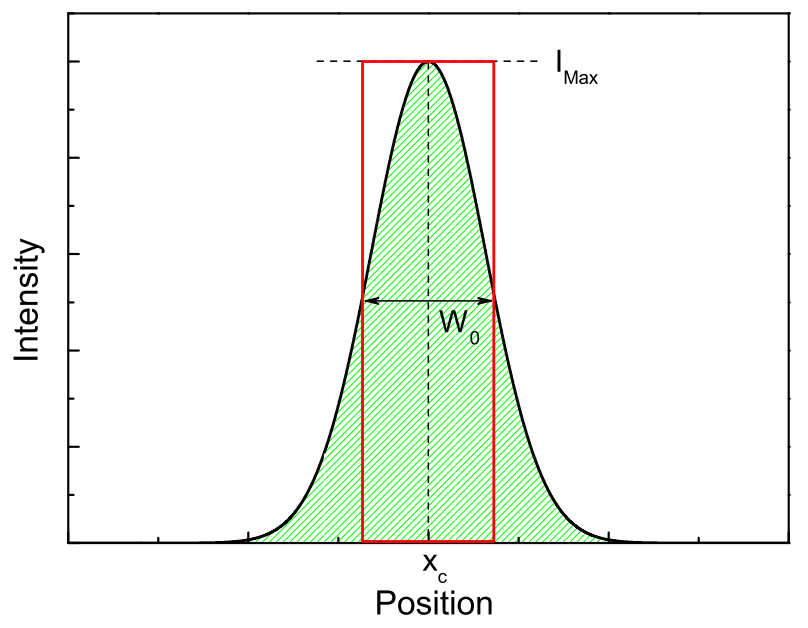

Figure B.2: A plot of the variation in the intensity with position for one dimension perpendicular to the incident light. The intensity follows a Gaussian function with a maximum intensity, $I_{\mathrm{Max}}$, at the centre of the beam, $x_{c}$. The effective width is then related to the width of the rectangle which has the same area as the Gaussian and height, $I_{\mathrm{Max}}$. This is the same as the FWHM of the Gaussian.

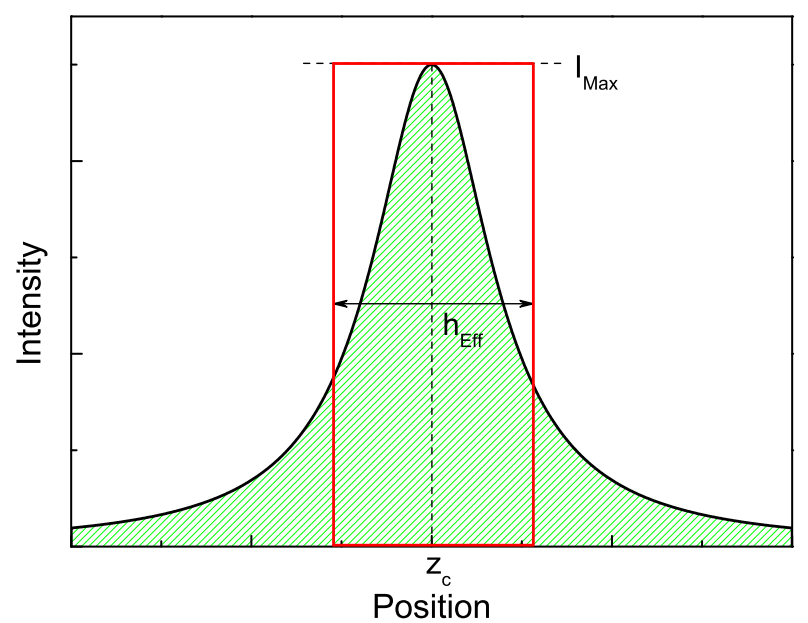

Figure B.3: A plot of the variation in the intensity with position for the dimension parallel to the incident light. The intensity follows a Lorentzian function with a maximum intensity, $I_{\mathrm{Max}}$, at the centre of the beam, $z_{c}$. The effective height is then given by the width of the rectangle which has the same area as the Lorentzian and height, $I_{\mathrm{Max}}$. 
(that is very close to a Lorentzian) such that [161]:

$$
h_{\mathrm{Eff}}=\frac{1}{I_{\mathrm{Max}}} \int_{-\infty}^{\infty} I(z) d z,
$$

where $I_{\text {Max }}$ is the intensity at the centre of the Lorentzian. The integral (the area under the Lorentzian) can be calculated by taking the sum of the intensities as a function of $\mathrm{z}$. 


\section{Appendix C}

\section{Measuring the SERS cross-section via a reference}

The cross-section is one of the most important properties of a molecule for SERS and yet one of the most difficult to measure. This is because the local field enhancement factor varies significantly between molecules. It is therefore very difficult to predict how many molecules actually contribute to the SERS signal. However, if the number of molecules is known, as is the case in single molecule SERS, the SERS cross-section can be estimated by comparing the SERS intensity with the Raman intensity of a molecule that has a known crosssection.

The average differential Stokes cross-section is related to the scattered intensity, $I_{S}$, and the incident power density, $P_{\text {Inc }}$ by (see section 2.3.3):

$$
I_{S}=N \frac{d \sigma_{S}}{d \Omega} P_{\text {Inc }}
$$

This is true in both surface-enhanced and normal Raman scattering. However, in the case of SERS the cross-section is an average over many local field enhancements, while in Raman scattering the cross-section is only averaged over the different orientations of the molecule (with respect to the incident light). If the experimental set-up is not changed between measurements, the ratio of the SERS intensity of one molecule and the Raman intensity of another molecule will be given by: 


$$
\frac{I_{\text {SERS }}}{I_{\text {Raman }}}=\frac{N_{\text {SERS }} d \sigma_{\text {SERS }} / d \Omega}{N_{\text {Raman }} d \sigma_{\text {Raman }} / d \Omega} .
$$

If the scattering volume, $V_{\text {Sca }}$, of the objective is known along with the molecular density, $n$, of the reference molecule (the molecule that we measure the normal Raman intensity of), we can rewrite this equation as:

$$
\frac{I_{\text {SERS }}}{I_{\text {Raman }}}=\frac{N_{\text {SERS }} d \sigma_{\text {SERS }} / d \Omega}{n V_{\text {Sca }} d \sigma_{\text {Raman }} / d \Omega} .
$$

If the Raman cross-section of one of the modes of the reference molecule is known, then we can estimate the SERS cross-section using this equation as long as we know the number of molecules contributing to the SERS signal. However, this is often not the case (which is why vibrational pumping was originally so attractive). Nevertheless, SERS cross-sections can be estimated under single molecule conditions $\left(N_{\mathrm{SERS}}=1\right)$ using this technique.

The choice of reference molecule will depend on the type of measurement that is performed. If dry samples are measured in air, the reference molecule will need to be a gas. In our experiments we take a Raman spectrum of air and measure the intensity of the $2330 \mathrm{~cm}^{-1}$ mode of nitrogen, which has a known cross-section of $16.4 \times 10^{-32} \mathrm{~cm}^{2} / \mathrm{sr}$ at $633 \mathrm{~nm}$ [177]. The number density of nitrogen in air can be easily estimated from the pressure and the fact that $78 \%$ (by volume) of air is made up of nitrogen. For an excitation wavelength, $\lambda$, other than $633 \mathrm{~nm}$, the cross-section will be modified by a factor of $\left(633 \times 10^{-9} \mathrm{~m}\right)^{4} / \lambda^{4}$.

For a liquid sample and an immersion objective, we typically use 2B2MP (2-bromo-2-methylpropane) as our reference molecule. At $633 \mathrm{~nm}$, the $516 \mathrm{~cm}^{-1}$ mode has a cross-section of $5.54 \times 10^{-30} \mathrm{~cm}^{2} / \mathrm{sr}[9,177]$. The cross-section can be modified for other excitation wavelengths in the same way as nitrogen. Typically we will use a concentration of $8.76 \mathrm{M}$ which can easily be converted to a number density. 


\section{Appendix D}

\section{Published Papers}

Below are the headers of the papers that have been published over the course of my $\mathrm{PhD}$ research.

\section{Polarization-dependent effects in surface-enhanced Raman scattering (SERS)}

P. G. Etchegoin, C. Galloway and E. C. Le Ru

Received 13th March 2006, Accepted 27th April 2006

First published as an Advance Article on the web 10th May 2006

DOI: $10.1039 / \mathbf{b 6 0 3 7 2 5 a}$

A few key examples of polarization effects in surface-enhanced Raman scattering (SERS) are highlighted and discussed. It is argued that the polarization of the local field, which is felt by an analyte molecule in a location of high electromagnetic field enhancement (hot-spot), can be very different from that of the incident exciting beam. The polarization dependence of the SERS signal

is, therefore, mostly dictated by the coupling of the laser to the plasmons rather than by the symmetry of the Raman tensor of the analyte. This sets serious restrictions for the interpretation of both single-molecule SERS polarization studies and for the use of circularly polarized light in techniques like surface-enhanced Raman optical activity.

Figure D.1: Physical Chemistry Chemical Physics, 8, 2624 (2006). Contribution: I performed the FemLab simulations and some of the GMT simulations for this paper. 
On the connection between optical absorption/extinction and SERS enhancements

E. C. Le Ru,* C. Galloway and P. G. Etchegoin

Received 12th April 2006, Accepted 30th May 2006

First published as an Advance Article on the web 8th June 2006

DOI: $10.1039 / \mathbf{b 6 0 5 2 9 2 d}$

Several aspects of the connection between the absorption/extinction spectra and the enhancement in surface enhanced Raman scattering (SERS) are analyzed and discussed. It is shown that in many standard situations the spatial distribution of the resonance plays a role for the difference between extinction/absorption and SERS enhancement and that the connection between both can be very indirect and, in many cases, misleading. This clarifies several misconceptions often found in the literature.

Figure D.2: Physical Chemistry Chemical Physics, 8, 3083 (2006). Contribution: I performed the FemLab simulations and some of the GMT simulations for this paper.

Vibrational pumping in surface enhanced Raman scattering (SERS)

R. C. Maher, ${ }^{* a}$ C. M. Galloway, ${ }^{b}$ E. C. Le Ru, ${ }^{b}$ L. F. Cohen ${ }^{a}$ and

P. G. Etchegoin $* b$

Received 7 th February 2008

First published as an Advance Article on the web 13th March 2008

DOI: $10.1039 / b 707870 f$

In this tutorial review, the underlying principles of vibrational pumping in surface enhanced

Raman scattering (SERS) are summarized and explained within the framework of their historical

development. Some state-of-the-art results in the field are also presented, with the aim of giving

an overview on what has been established at this stage, as well as hinting at areas where future

developments might take place.

Figure D.3: Chemical Society Reviews, 37,965 (2008). Contribution: I Performed the two laser pumping experiments and analysis that was used in this paper. 


\title{
Single-molecule vibrational pumping in SERS
}

\author{
C. M. Galloway, E. C. Le Ru* and P. G. Etchegoin*
}

Received 6th March 2009, Accepted 28th May 2009

First published as an Advance Article on the web 29th June 2009

DOI: $10.1039 / \mathrm{b} 904638 \mathrm{k}$

Single-molecule vibrational pumping in surface-enhanced Raman scattering (SERS) is demonstrated rigorously using the bi-analyte SERS method at low temperatures. These experiments reveal a systematic difference between the radiative SERS cross section estimated from the Stokes intensity and that obtained by pumping itself (from the anti-Stokes-to-Stokes ratio), the latter being always larger. This difference can only be reliably demonstrated in the single-molecule SERS regime, for it is otherwise affected by complications of the averaging (over the enhancement distribution) of the signals of several molecules. The findings in this paper highlight the limitations of the pumping cross-section, which cannot (in general) be taken as a reliable measure of the SERS cross-section itself. We provide a discussion of the main possible explanations for the systematic difference of the two estimates.

Figure D.4: Physical Chemistry Chemical Physics, 11 7372 (2009). Contribution: I Performed all of the experiments along with the analysis and wrote the first draft.

Ultrafast Nonradiative Decay Rates on Metallic Surfaces by Comparing Surface-Enhanced Raman and Fluorescence Signals of Single Molecules

C. M. Galloway, P. G. Etchegoin,** and E.C. Le Ru ${ }^{\dagger}$

The MacDiarmid Institute for Advanced Materials and Nanotechnology, School of Chemical and Physical Sciences, Victoria University of Wellington, PO Box 600, Wellington, New Zealand

(Received 30 June 2009; published 5 August 2009)

By the simultaneous observation of surface-enhanced Raman scattering and surface-enhanced fluores cence signals from a single molecule, we can measure and quantify the modification of the total decay rate of emitters in very close proximity to metals, even down to adsorbed molecules. This modified decay rate is shown to be largely dominated by its nonradiative component, which would be extremely difficult to estimate with conventional approaches. The method provides an indirect measurement of ultrafast ( $\sim 25 \mathrm{fs})$ mechanisms, which would be impossible to gain with time-resolved spectroscopy of a single molecule.

Figure D.5: Physical Review Letters, 103, 063003 (2009). Contribution: I Performed all of the experiments along with most of the analysis. 


\section{An Iterative Algorithm for Background Removal in Spectroscopy by Wavelet Transforms}

C. M. GALloWAY,* E. C. LE RU, and P. G. ETCHEGOIN

The MacDiarmid Institute for Advanced Materials and Nanotechnology, School of Chemical and Physical Sciences, Victoria University of The MacDiarmid Institute for Advanced Materials
Wellington, PO Box 600 Wellington, New Zealand

Wavelet transforms are an extremely powerful tool when it comes to processing signals that have very "low frequency" components or nonperiodic events. Our particular interest here is in the ability of wavelet transforms to remove backgrounds of spectroscopic signals. We will discuss the case of surface-enhanced Raman spectroscopy (SERS) for illustration, but the situation it depicts is widespread throughout myriad different types of spectroscopies (IR, NMR, etc.). We outline a purposebuilt algorithm that we have developed to perform an iterative wavelet transform. In this algorithm, the effect of the signal peaks above the background is reduced after each iteration until the fit converges close to the real background. Experimental examples of two different SERS applications are given: one involving broad backgrounds (that do not vary much among spectra), and another that involves single molecule SERS (SM-SERS) measurements with narrower (and varying) backgrounds. In both cases, we will show that wavelet transforms can be used to fit the background with a great deal of accuracy, thus providing the framework for automatic background removal of large sets of data (typically obtained in time-series or spatial mappings). A MATLAB based application that utilizes the iterative algorithm developed here is freely available to download from http://www.victoria.ac.nz/raman/publis/codes/cobra.aspx. Index Headings: Wavelet transform; Background subtraction; Raman spectroscopy; Data processing; Surface-enhanced Raman spectroscopy; SERS.

backgrounds (from impurities or additional compounds). If the background is very similar from point to point in the mapping, a relatively easy background subtraction for all of them could be achieved before the linear decomposition in terms of the reference spectra is performed. However, if the background changes randomly from point to point, a more reliable and efficient background subtraction routine is needed: one that will not introduce artifacts in the process of subtracting the background and that can be carried out for hundreds (sometimes thousands, or tens of thousands) of spectra. A few tens of spectra can be analyzed by hand, but it is unlikely that tens of thousands of spectra (in a Raman map or a time series, for example) can be analyzed in this way. SERS spectra (and single molecule (SM) SERS in particular, to which this (application is applica is parictible to (1) event in either mappings or time seies (in pre-resonant dye molecules, these backgrounds can be attributed to surface-enhanced fluorescence, ${ }^{2}$ whose spectral profile is modified by the underlying plasmon resonance dispersion. ${ }^{3}$ The combination of large numbers of spectra and

Figure D.6: Applied Spectroscopy, 63, (2009). Contribution: I Performed all of the coding for the application and the analysis of its effectiveness. I also wrote the paper and made modifications based on the referees comments. 


\section{Bibliography}

[1] R. C. Maher. Surface Enhanced Raman Scattering. PhD thesis, University of London, Imperial College, 2007.

[2] C. M. Galloway, E. C. Le Ru, and P. G. Etchegoin. An Iterative Algorithm for Background Removal in Spectroscopy by Wavelet Transforms. Applied Spectroscopy, 63(12), 2009. to be released December 2009.

[3] C. M. Galloway, E. C. Le Ru, and P. G. Etchegoin. Single-Molecule Vibrational Pumping in SERS. Physical Chemistry Chemical Physics, 11: $7372,2009$.

[4] K. Kneipp, Y. Wang, H. Kneipp, I. Itzkan, R. R. Dasari, and M. S. Feld. Population Pumping of Excited Vibrational States by Spontaneous Surface-Enhanced Raman Scattering. Physical Review Letters, 76(14): 2444, 1996.

[5] R. C. Maher, L. F. Cohen, J. C. Gallop, E. C. Le Ru, and P. G. Etchegoin. Temperature-Dependent Anti-Stokes/Stokes Ratios under Surface-Enhanced Raman Scattering Conditions. J. Phys. Chem. B, 110: 6797-6803, 2006.

[6] R. C. Maher, L. F. Cohen, E. C. Le Ru, and P. G. Etchegoin. A study of local heating of molecules under Surface Enhanced Raman Scattering (SERS) conditions using the anti-Stokes/Stokes ratio. Faraday Discussions, 132:77, 2006.

[7] R. C. Maher, L. F. Cohen, E. C. Le Ru, and P. G. Etchegoin. On the Experimental Estimation of Surface Enhanced Raman Scattering (SERS) Cross Sections by Vibrational Pumping. J. Phys. Chem. B, 110:1946919478, 2006. 
[8] R. C. Maher, P. G. Etchegoin, E. C. Le Ru, and L. F. Cohen. A Conclusive Demonstration of Vibrational Pumping under Surface Enhanced Raman Scattering Conditions. J. Phys. Chem. B, 110:11757-11760, 2006.

[9] P. G. Etchegoin and E. C. Le Ru. Principles of Surface-Enhanced Raman Spectroscopy and Related Plasmonic Effects. Elsevier, Amsterdam, 2009.

[10] W. Demtröder. Laser Spectroscopy. Springer, Berlin, 2003.

[11] H. Haken, H. C. Wolf, and W. D. Brewer. Molecular Physics and Elements of Quantum Chemistry: Introduction to Experiments and Theory. Springer-Verlag, Berlin, 2004.

[12] F. Nogueira, A. Castro, and M. A. L. Marques. A Tutorial on Density Functional Theory, volume 620 of Lecture Notes in Physics. Springer Berlin / Heidelberg, Berlin, 2003. 218-256 pp.

[13] R. M. Dreizler and E. K. U. Gross. Density Functional Theory: An Approach to the Quantum Many-Body Problem. Springer-Verlag, Berlin, 1990.

[14] J. Neugebauer, M. Reiher, C. Kind, and B. A. Heiss. Quantum chemical calculation of vibrational spectra of large molecules - Raman and IR spectra for Buckminsterfullerene. J. Computational Chem., 23:895-910, 2002.

[15] V. Kozich, J. Dreyer, S. Ashihara, W. Werncke, and T. Elsaesser. Modeselective $\mathrm{OH}$ stretching relaxation in a hydrogen bond studied by ultrafast vibrational spectroscopy. The Journal of Chemical Physics, 125:074504, 2006.

[16] L. Rayleigh. On the scattering of light by small particles. Philosophical Magazine, page 274, 1871.

[17] C. V. Raman and K. S. Krishnan. A New Type of Secondary Radiation. Nature, 121:501, 1928.

[18] G. Glockler. The Raman Effect. Reviews of Modern Physics, 15(2):111, 1943.

[19] D. A. Long. The Raman Effect: A Unified Treatment of the Theory of Raman Scattering by Molecules. John Wileys \& Sons, 2002. 
[20] B. Schrader and D. S. Moore. Laser-Based Molecular Spectroscopy for Chemical Analysis-Raman Scattering Processes. Pure and Applied Chemistry, 69(7):1451, 1997.

[21] J. D. Jackson. Classical Electrodynamics. Wiley, New York, 3 edition, 1998.

[22] H. Watanabe, N. Hayazawa, Y. Inouye, and S. Kawata. DFT Vibrational Calculations of Rhodamine 6G Adsorbed on Silver: Analysis of Tip-Enhanced Raman Spectroscopy. Journal of Physical Chemistry B, 109:5012, 2005.

[23] J. G. Fujimoto and T. K. Yee. Diagrammatic Analysis of Third Order Nonlinear Optical Processes. Journal of Quantum Electronics, 19(5):861, 1983.

[24] C. K. Johnson and S. A. Soper. Nonlinear Surface-Enhanced Spectroscopy of Silver Colloids and Pyridine: Hyper-Raman and Second-Harmonic Scattering. The Journal of Physical Chemistry, 93(21):7281, 1989.

[25] T. Itoh, Y. Ozaki, H. Yoshikawa, T. Ihama, and H. Masuhara. HyperRayleigh scattering and hyper-Raman scattering of dye-adsorbed silver nanoparticles induced by a focused continuous-wave near-infrared laser. Appl. Phys. Lett., 88:084102, 2006.

[26] B. Valeur. Molecular Fluorescence. Principles and Applications. WileyVCH, Weinheim, 2002.

[27] R. M. Dickson, A. B. Cubitt, R. Y. Tsien, and W. E. Moerner. On/off blinking and switching behavior of single molecules of green fluorescent protein. Nature (London), 388:355-358, 1997.

[28] J. K. Trautman, J. J. Macklin, L. E. Brus, and E. Betzig. Near-field spectroscopy of single molecules at room temperature. Nature (London), 369:40-2, 1994.

[29] J. Widengren, Ãœ. Mets, and R. Rigler. Fluorescence spectroscopy of triplet states in solution: a theoretical and experimental study. J. Phys. Chem., 99(36):13368-13379, 1995.

[30] J. Widengren and R. Rigler. Mechanisms of photobleaching investigated by fluorescence correlation spectroscopy. Bioimaging, 4(3):149-157, 1996. 
[31] M. G. Albrecht and J. A. Creighton. Anomalously Intense Raman Spectra of Pyridine at a Silver Electrode. Journal of the American Chemical Society, 99(15):5215, 1977.

[32] M. Fleischmann, P. J. Hendra, and A. J. McQuillan. Raman Spectra of Pyridine Adsorbed at a Silver Electrode. Chemical Physics Letters, 26(2): 163, 1974.

[33] R. Aroca. Surface Enhanced Vibrational Spectroscopy. Wiley, Chichester, 2006.

[34] C. L. Haynes, A. D. McFarland, and R. P. Van Duyne. Surface Enhanced Raman Spectroscopy. Analytical Chemistry, 77:339A-346A, 2005.

[35] D. L. Jeanmaire and R. P. Van Duyne. Surface Raman Spectroelectrochemistry. Journal of Electroanalytical Chemistry, 84:1, 1977.

[36] H. Metiu. Surface enhanced spectroscopy. Progress in Surface Science, 17:153, 1984 .

[37] M. Moskovits. Surface-enhanced spectroscopy. Reviews of Modern Physics, 57(3):783, 1985.

[38] A. Otto. Light Scattering in Solids IV, volume 54. Springer-Verlag, Berlin, 1984.

[39] D. Pines. Collective Energy Losses in Solids. Reviews of Modern Physics, 28(3):184, 1956.

[40] S. A. Maiera and H. A. Atwater. Plasmonics: Localization and guiding of electromagnetic energy in metal/dielectric structures. Applied Physics Reviews, 98:011101, 2005.

[41] L. Novotny and B. Hecht. Principles of Nano-Optics. Cambridge University Press, Cambridge,, 2006.

[42] A. V. Zayats, I. I. Smolyaninov, and A. A. Maradudin. Nano-optics of surface plasmon polaritons. Physics Reports, 408:131, 2005.

[43] R. H. Ritchie. Plasma Losses by Fast Electrons in Thin Films. Physical Review, 106(5):874, 1957. 
[44] C. J. Powell and J. B. Swan. Origin of the Characteristic Electron Energy Losses in Aluminium. Physical Review, 115(4):869, 1959.

[45] E. A. Stern and R. A. Ferrell. Surface Plasma Oscillations of a Degenerate Electron Gas. Physical Review, 120(1):130, 1960.

[46] R. M. Jarvis, H. E. Johnson, E. Olembe, A. Panneerselvam, M. A. Malik, M. Afzaal, P. OBrien, and R. Goodacre. Towards Quantitatively Reproducible Substrates for SERS. Analytical Chemistry, 133:1449, 2008.

[47] E.C. Le Ru, M. Dalley, and P.G. Etchegoin. Plasmon resonances of silver colloids studied by surface enhanced Raman spectroscopy. Current Applied Physics, 6:411, 2006.

[48] M. Meyer, E. C. Le Ru, and P. G. Etchegoin. Self-Limiting Aggregation Leads to Long-Lived Metastable Clusters in Colloidal Solutions. The Journal of Physical Chemistry B, 110:6040, 2006.

[49] L. Rodríguez-Lorenzo, R. A. Álvarez-Puebla, I. Pastoriza-Santos, S. Mazzucco, O. Stéphan, M. Kociak, L. M. Liz-Marzán, and F. J. G. de Abajo. Zeptomol Detection Through Controlled Ultrasensitive Surface-Enhanced Raman Scattering. Journal of the American Chemical Society, 131:4616, 2009.

[50] R. Rojas and F. Claro. Theory of surface enhanced Raman scattering in colloids. The Journal of Chemical Physics, 98(2):998, 1993.

[51] Z. B. Wang, B. S. Luk'yanchuk, W. Guo, S. P. Edwardson, D. J. Whitehead, L. Li, Z. Liu, and K. G. Watkins. The influences of particle number on hot spots in strongly coupled metal nanoparticles chain. The Journal of Chemical Physics, 128:094705, 2008.

[52] L. Aigouy, V. Mathet, and P. Beauvillain. Electromagnetic Field distribution on a rough gold thin film: An experimental study as a function of the gold thickness. Optics Communications, 262(2):263, 2006.

[53] S. Buil, J. Aubineau, J. Laverdant, and X. Qulin. Local field intensity enhancements on gold semicontinuous films investigated with an aperture nearfield optical microscope in collection mode. Journal of Applied Physics, 100:063530, 2006. 
[54] W. Zhang, B. S. Yeo, T. Schmid, and R. Zenobi. Single Molecule TipEnhanced Raman Spectroscopy with Silver Tips. The Journal of Physical Chemistry C, 111(4):1733, 2007.

[55] C. L. Du, Y. M. You, J. Kasim, C. P. Wong H. M. Fan Z. H. Ni, T. Yu, and Z. X. Shen. Confocal White Light Reflection Imaging for Characterization of Metal Nanostructures. Optics Communications, 281:5360, 2008.

[56] S. Mahajan, M. Abdelsalam, Y. Suguwara, S. Cintra, A. Russel, J. Baumberg, and P. Bartlett. Tuning plasmons on nano-structured substrates for NIR-SERS. Phys. Chem. Chem. Phys., 9:104, 2007.

[57] T. Rindzevicius, Y. Alaverdyan, B. Sepulveda, T. Pakizeh, M. Kall, R. Hillenbrand, J. Aizpurua, and F. J. G. de Abajo. Nanohole Plasmons in Optically Thin Gold Films. The Journal of Physical Chemistry C, 111: $1207,2007$.

[58] C. Noguez. Surface Plasmons on Metal Nanoparticles: The Influence of Shape and Physical Environment. Journal of Physical Chemistry C, 111: $3806,2007$.

[59] E. C. Le Ru, C. Galloway, and P. G. Etchegoin. On the connection between optical absorption/extinction and SERS enhancements. Physical Chemistry Chemical Physics, 8:3083, 2006.

[60] P. B. Johnson and R. W. Christy. Optical Constants of the Noble Metals. Physical Review B, 6(12):4370, 1972.

[61] E. D. Palik, editor. Hanbook of Optical Constants of Solids. Academic Press, 1998

[62] P. G. Etchegoin, E. C. Le Ru, and M. Meyer. An Analytical Model for the Optical Properties of Gold. The Journal of Chemical Physics, 125: 164705, 2006.

[63] G. W. Ford. Electromagnetic Interactions of Molecule with Metal Surfaces. Physics Reports, 113:195, 1984.

[64] M. Futamata and Y. Maruyama. Electromagnetic and chemical interaction between Ag nanoparticles and adsorbed rhodamine molecules in surface-enhanced Raman scattering. Anal. bioanal. chem., 388:89, 2007. 
[65] Q. Zhou, G. Zhao, Y. Chao, Y. Li, Y. Wu, and J. Zheng. Charge-Transfer Induced Surface-Enhanced Raman Scattering in Silver Nanoparticle Assemblies. J. Phys. Chem. C, 111(5):1951, 2007.

[66] K. Yoshida, T. Itoh, V. Biju, M. Ishikawa, and Y. Ozaki. Experimental evaluation of the twofold electromagnetic enhancement theory of surfaceenhanced resonance Raman scattering. Physical Review B, 79:085419, 2009.

[67] T. Itoh, K. Yoshida, V. Biju, Y. Kikkawa, M. Ishikawa, and Y. Ozaki. Second enhancement in surface-enhanced resonance Raman scattering revealed by an analysis of anti-Stokes and Stokes Raman spectra. Physical Review B, 76:085405, 2007.

[68] E.C. Le Ru and P.G. Etchegoin. Rigorous justification of the $|E|^{4}$ enhancement factor in Surface Enhanced Raman Spectroscopy. Chemical Physics Letters, 423(1-3):63, 2006.

[69] P. Anger, P. Bharadwaj, and L. Novotny. Enhancement and Quenching of Single-Molecule Fluorescence. Physical Review Letters, 96:113002, 2006.

[70] E. Dulkeith, A. C. Morteani, T. Niedereichholz, , T. A. Klar, J Feldmann, S. A. Levi, F. C. J. M. van Veggel, D. N. Reinhoudt, M. Möller, and D. I. Gittins. Fluorescence Quenching of Dye Molecules near Gold Nanoparticles: Radiative and Nonradiative Effects. Physical Review Letters, 89: 203002, 2002.

[71] S. Kühn, U. Håkanson, L. Rogobete, and V. Sandoghdar. Enhancement of Single-Molecule Fluorescence Using a Gold Nanoparticle as an Optical Nanoantenna. Physical Review Letters, 97:017402, 2006.

[72] E. C. Le Ru, J. Grand, N. Félidj, J. Aubard, G. Lévi, A. Hohenau, J. R. Krenn, E. Blackie, and P. G. Etchegoin. Experimental Verification of the SERS Electromagnetic Model beyond the - E- ${ }^{4}$ Approximation: Polarization Effects. The Journal of Physical Chemistry C, 112:8117, 2008.

[73] E. C. Le Ru, P. G. Etchegoin, N. Félidj, J. Grand, G. Lévi, and J. Aubard. Mechanisms of Spectral Profile Modification in Surface-Enhanced Fluorescence. The Journal of Physical Chemistry C, 111:16076, 2007.

[74] P. Bharadwaj and L. Novotny. Spectral Dependence of Single Molecule Fluorescence Enhancement. Optics Express, 15(21):14266, 2007. 
[75] R. Carminati, J.-J. Greffet, C. Henkel, and J. M. Vigoureux. Radiative and non-radiative decay of a single molecule close to a metallic nanoparticle. Optics Communications, 261:368, 2006.

[76] P. Johansson, H. Xu, and M. Kall. Surface-enhanced Raman scattering and fluorescence near metal nanoparticles. Physical Review B, 72:035427, 2005.

[77] H. Xu, X. Wang, M. P. Persson, H.Q. Xu, M. Kall, and P. Johansson. Unified Treatment of Fluorescence and Raman Scattering Processes near Metal Surfaces. Physical Review Letters, 93:243002, 2004.

[78] M. Ringler, A. Schwemer, M. Wunderlich, A. Nichtl, K. Kürzinger, T. A. Klar, and J. Feldmann. Shaping Emission Spectra of Fluorescent Molecules with Single Plasmonic Nanoresonators. Physical Review Letters, 100:203002, 2008.

[79] A. Wokaun, J. P. Gordon, and P. F. Liao. Radiation Damping in SurfaceEnhanced Raman Scattering. Physical Review Letters, 48(14):957, 1982.

[80] M. Meier and A. Wokaun. Enhanced Fields on Large Metal Particles: Dynamic Depolarization. Optical Letters, 8:581, 1983.

[81] J. M. Gérardy and M. Ausloos. Absorption spectrum of clusters of spheres from the general solution of Maxwell's equations. II. Optical properties of aggregated metal spheres. Physical Review B, 25(6):4204, 1982.

[82] http://www.comsol.com/.

[83] T. Itoh, K. Hashimoto, and Y. Ozakia. Polarization dependences of surface plasmon bands and surface-enhanced Raman bands of single Ag nanoparticles. Applied Physics Letters, 83(11):2274, 2003.

[84] T. Shegai, Z. Li, T. Dadosh, Z. Zhang, H. Xu, and G. Haran. Managing light polarization via plasmonmolecule interactions within an asymmetric metal nanoparticle trimer. Proceedings of the National Academy of Sciences of the United States of America, 105(43):16448, 2008.

[85] P. G. Etchegoin, C. Galloway, and E. C. Le Ru. Polarization-dependent effects in surface-enhanced Raman scattering (SERS). Physical Chemistry Chemical Physics, 8:2624, 2006. 
[86] E. C. Le Ru, P. G. Etchegoin, and M. Meyer. Enhancement factor distribution around a single surface-enhanced Raman scattering hot spot and its relation to single molecule detection. The Journal of Chemical Physics, 125:204701, 2006.

[87] P. G. Etchegoin, M. Meyer, and E. C. Le Ru. Statistics of single molecule SERS signals: is there a Poisson distribution of intensities? Physical Chemistry Chemical Physics, 9:1, 2007.

[88] E. C. Le Ru and P. G. Etchegoin. Vibrational pumping and heating under SERS conditions: fact or myth? Faraday Discussions, 132:63, 2006.

[89] A. G. Brolo, A. C. Sanderson, and A. P. Smith. Ratio of the surfaceenhanced anti-Stokes scattering to the surface-enhanced Stokes-Raman scattering for molecules adsorbed on a silver electrode. Physical Review B, 69:045424, 2004 .

[90] T. L. Haslett, L. Tay, and M. Moskovits. Can surface-enhanced Raman scattering serve as a channel for strong optical pumping? Journal of Chemical Physics, 113(4):1641, 2000.

[91] P. V. Teredesai, A. K. Sood, A. Govindaraj, and C. N. R. Rao. Surface enhanced resonance Raman scattering from radial and tangential modes of semiconducting single wall carbon nanotubes. Appl. Surf. Sci., 182(3): 196, 2001.

[92] R. C. Maher, C. M. Galloway, E. C. Le Ru, L. F. Cohen, and P. G. Etchegoin. Vibrational pumping in surface enhanced Raman scattering (SERS). Chemical Society Reviews, 37:965, 2008.

[93] R. C. Maher, J. Hou, L. F. Cohen, E. C. Le Ru, J. M. Hadfield, J. E. Harvey, P. G. Etchegoin, F. M. Liu, M. Green, R. J. C. Brown, and M. J. T. Milton. Resonance contributions to anti-Stokes/Stokes ratios under surface enhanced Raman scattering conditions. The Journal of Chemical Physics, 123:084702, 2005.

[94] A. Laubereau and W. Kaiser. Vibrational dynamics of liquids and solids investigated by picosecond light pulses. Review of Modern Physics, 50: 607, 1978.

[95] A. Lagendijk. Ultrashort Processes in Condensed Matter. Springer, 1993. 197-238 pp. 
[96] F. W. Wise, M. J. Rosker, and C. L. Tang. Oscillatory femtosecond relaxation of photoexcited organic molecules. J. Chem. Phys., 88(5):2827, 1987.

[97] Q. Zhong, Z. Wang, Y. Sun, Q. Zhu, and F. Kong. Vibrational relaxation of dye molecules in solution studied by femtosecond time-resolved stimulated emission pumping fluorescence depletion. Chemical Physics Letters, 248:277, 1996.

[98] Y. Nagasawa, Y. Ando, D. Kataoka, H. Matsuda, H. Miyasaka, and T. Okada. Ultrafast Excited State Deactivation of Triphenylmethane Dyes. The Journal of Physical Chemistry A, 106(10):2024, 2002.

[99] Q. Hong, I. A. D. Pexton, G. Porter, and D. R. Klug. Dephasing of Excited-State Wave Packets in an Oxazine Dye. J. Phys. Chem., 97: 12561-12565, 1993.

[100] R. M. Shelby, C. B. Harris, and P. A. Cornelius. The origin of vibrational dephasing of polyatomic molecules in condensed phases. J. Chem. Phys., $70(1): 34,1979$.

[101] P. G. Etchegoin and E. C. Le Ru. Resolving single molecules in SERS within the inhomogeneous broadening of Raman peaks. Journal of American Chemical Society, 2009. Yet to be submitted.

[102] P. G. Etchegoin, E. C. Le Ru, R. C. Maher, and L. F. Cohen. Enhancement factor averaging and the photostability of probes in SERS vibrational pumping. Physical Chemistry Chemical Physics, 9(35):1463, 2007.

[103] http://www.horiba.com/fr/scientific/products/ramanspectroscopy/accessories/labspec-software/.

[104] P. C. Lee and D. Meisel. Absorption and Surface-Enhanced Raman of Dyes on Silver and Gold Sols. The Journal of Chemical Physics, 86:3391, 1982.

[105] J. J. Turner. Handbook of Vibrational Spectroscopy, volume 1. John Wiley \& Sons, New York, 2002.

[106] M. J. Clouter, H. Kiefte, and R. K. Jain. Pure vibrational Raman spectra of simple liquids: $\mathrm{O}_{2}, \mathrm{~N}_{2}, \mathrm{CO}, \mathrm{CH}_{4}, \mathrm{CF}_{4}$. The Journal of Chemical Physics, 73(2):673, 1980. 
[107] C. Manzanares and G. E. Ewing. Vibrational relaxation of small molecules in the liquid phase:Liquid nitrogen doped with $\mathrm{H}_{2}$ or $\mathrm{D}_{2}$. Journal of Chemical Physics, 69(6):2803, 1978.

[108] S. Marks, P. A. Cornelius, and C. B. Harris. A critical test of vibrational dephasing theories in solids using spontaneous Raman scattering in isotopically mixed crystals. J. Chem. Phys., 73(7):3069, 1980.

[109] E. C. Le Ru, M. Meyer, and P. G. Etchegoin. Proof of Single-Molecule Sensitivity in Surface Enhanced Raman Scattering (SERS) by Means of a Two-Analyte Technique. Journal of Physical Chemistry B, 110:1944, 2006.

[110] J. Sarkar, J. Chowdhury, P. Pal, and G. B. Talapatr. Ab initio, DFT vibrational calculations and SERRS study of Rhodamine 123 adsorbed on colloidal silver particles. Vibrational Spectroscopy, 41(1):90, 2006.

[111] C. L. Brooks III, M. Karplus, and B. M. Pettitt. Advances in Chemical Physics. John Wiley \& Sons, 1988.

[112] S. Naumov, S. Kapoor, S Thomas, S. Venkateswaran, and T. Mukherjee. SERS of benzotriazole on Ag colloid: surface structure characterization using the DFT approach. Journal of Molecular Structure:THEOCHEM, $685: 127,2004$.

[113] O. Madelung. Introduction to Solid State Theory. Springer-Verlag, Berlin, 1978.

[114] G. Deinzer, M. Schmitt, A. P. Mayer, and D. Strauch. Intrinsic lifetimes and anharmonic frequency shifts of long-wavelength optical phonons in polar crystals. Physical Review B, 69:014304, 2004.

[115] J. Serrano, M. Cardona, T. M. Ritter, B. A. Weinstein, A. Rubio, and C. T. Lin. Pressure and temperature dependence of the Raman phonons in isotopic $\gamma$-CuI. Physical Review B, 66:245202, 2002.

[116] N. M. Gasanly. Effect of crystal disorder on linewidth of the Raman modes in $\mathrm{GaS}_{1-x} \mathrm{Se}_{x}$ layered mixed crystals. Crystal Research and Technology, 38(11):962, 2003. 
[117] R. Kroon, R. Sprik, and A. Lagendijk. Picosecond vibrational relaxation modulated by critical slowing down in ferroelectric $\mathrm{K}_{4} \mathrm{Fe}(\mathrm{CN})_{6} 3 \mathrm{H}_{2} 0$. Physical Review B, 42(5):2785, 1990.

[118] D. Hsu and J. L. Skinner. Nonperturbative theory of temperaturedependent optical dephasing in crystals. I. Accoustic or optical phonons. The Journal of Chemical Physics, 81(12):5471, 1984.

[119] D. Hsu and J. L. Skinner. On the thermal broadening of zero-phonon impurity lines in absorption and fluorescence spectra. The Journal of Chemical Physics, 81(4):1604, 1984.

[120] D. Hsu and J. L. Skinner. Nonperturbative theory of temperaturedependent optical dephasing in crystals. IV. Microscopic model for pseudolocal phonons. The Journal of Chemical Physics, 87(1):54, 1987.

[121] B. B. Laird and J. L. Skinner. $\mathrm{T}_{2}$ can be greater than $2 \mathrm{~T}_{1}$, even at finite temperature. The Journal of Chemical Physics, 94(6):4405, 1991.

[122] K. E. Jones and A. H. Zewail. Advances in Laser Chemistry. Springer, New York, 1978.

[123] G. J. Small. Comment on frequency shift and transverse relaxation of optical transitions in organic solids. Chemical Physics Letter, 57(4):501, 1978.

[124] C. B. Harris, R. M. Shelby, and P. A. Cornelius. Effects of Energy Exchange of Vibrational Dephasing Times in Raman Scattering. Physical Review Letters, 38(24):1415, 1977.

[125] J. Vgh. Alternative form for the pseudo-Voigt peak shape. Review of Scientific Instruments, 76:056107, 2005.

[126] H. Du, R. A. Fuh, J. Li, A. Corkan, and J. S. Lindsey. PhotochemCAD: A Computer-Aided Design and Research Tool in Photochemistry. Photochemistry and Photobiology, 68(2):141, 1998.

[127] J. A. Dieringer, A. D. McFarland, N. C. Shah, D. A. Stuart, A. V. Whitney, C. R. Yonzon, M. A. Young, X. Zhang, and R. P. Van Duyne. Surface enhanced Raman spectroscopy: new materials, concepts, characterization tools, and applications. Faraday Discussions, 132:9, 2006. 
[128] I. Baltog, M. Baibarac, and S. Lefrant. Single-beam pumped Coherent anti-Stokes Raman scattering on carbon nanotubes thin films excited through surface plasmons I. Physica E, 40(7):2380, 2007.

[129] J.K. Brasseur, P.A. Roos, K.S. Repasky, and J.L. Carlsten. Coherent Anti-Stokes Emission in a Continuous Wave Raman Laser in $\mathrm{H}_{2}$. Journal of the Optical Society of America B: Optical Physics, 17(7):1223, 2000.

[130] http://www.victoria.ac.nz/raman/publis/codes/codes.aspx.

[131] I. Daubechies. Orthonormal bases of compactly supported wavelets. Communications of Pure and Applied Mathematics, 41:909, 1988.

[132] S. G. Mallat. A Theory for Multiresolution Signal Decomposition: The Wavelet Representation. IEEE Transactions on Pattern Analysis and Machine Intelligence, 11(7):674, 1989.

[133] K. B. Alsberg, A. M. Woodward, and D. B. Kell. An introduction to wavelet transforms for chemometricians: A time-frequency approach. Chemometrics and Intelligent Laboratory Systems, 37:215, 1997.

[134] F. Ehrentreich. Wavelet transform applications in analytical chemistry. Analytical and Bioanalytical Chemistry, 372:115, 2002.

[135] K. Jetter, U. Depczynski, K. Molt, and A. Niemller. Principles and applications of wavelet transformation to chemometrics. Analytica Chimica Acta, 420:169, 2000.

[136] X. G. Shao, A. K. Leung, and F. T. Chau. Wavelet: A New Trend in Chemistry. Accounts of Chemical Research, 36:276, 2003.

[137] B. Walczak and D.L. Massart. Wavelets - something for analytical chemistry? Trends in Analytical Chemistry, 16(8):451, 1997.

[138] E. Dinc and D. Baleanu. Multidetermination of thiamine $\mathrm{HCl}$ and pyridoxine $\mathrm{HCl}$ in their mixture using continuous daubechies and biorthogonal wavelet analysis. Talanta, 59:707, 2003.

[139] X. Shao, C. Pang, and Q. Su. A novel method to calculate the approximate derivative photoacoustic spectrum using continuous wavelet transform. Fresenius' Journal of Analytical Chemistry, 367:525, 2000. 
[140] X. Shao, L. Shao, and G. Zhao. Extraction of extended X-ray absorption fine structure information from the experimental data using the wavelet transform. Analytical Communications, 35:135, 1998.

[141] W. Cai, L. Wang, Z. Pan, J. Zuo, C. Xu, and X. Shao. Application of the wavelet transform method in quantitative analysis of Raman spectra. Journal of Raman Spectroscopy, 32:207, 2001.

[142] Y. Hu, T. Jiang, A. Shen, W. Li, X. Wang, and J. Hu. A background elimination method based on wavelet transform for Raman spectra. Chemometrics and Intelligent Laboratory Systems, 85:94, 2007.

[143] P. M. Ramos and I. Ruisanchez. Noise and background removal in Raman spectra of ancient pigments using wavelet transform. Journal of Raman Spectroscopy, 36:848, 2005.

[144] C. K. Chui. An Introduction to Wavelets. Academic Press, 1992.

[145] I. Daubechies. Ten Lectures on Wavelets. Society for Industrial and Applied Mathematics, 1992.

[146] A. K. Leung, F. Chau, and J. Gao. A review on applications of wavelet transform techniques in chemical analysis:1989-1997. Chemometrics and Intelligent Laboratory Systems, 43:165, 1998.

[147] M. Misiti, Y. Misiti, G. Oppenheim, and J Poggi. Wavelet Toolbox ${ }^{T M} 4$ Users Guide.

[148] I. Daubechies. The Wavelet Transform, Time-Frequency Localization and Signal Analysis. IEEE Transactions on Information Theory, 36(5):961, 1990.

[149] V. J. Barclay, R. F. Bonner, and I. P. Hamilton. Application of Wavelet Transforms to Experimental Spectra: Smoothing, Denoising, and Data Set Compression. Analytical Chemistry, 69:78, 1997.

[150] C. Cai and P. B. Harrington. Different Discrete Wavelet Transforms Applied to Denoising Analytical Data. Journal of Chemical Information and Computer Sciences, 38:1161, 1998.

[151] F. Ehrentreich and L. Summchen. Spike Removal and Denoising of Raman Spectra by Wavelet Transform Methods. Analytical Chemistry, 73:4364, 2001. 
[152] B. Walczak and D.L. Massart. Noise suppression and signal compression using the wavelet packet transform. Chemometrics and Intelligent Laboratory Systems, 36:81, 1997.

[153] C. Perrin, B. Walczak, and D. Luc Massart. The Use of Wavelets for Signal Denoising in Capillary Electrophoresis. Analytical Chemistry, 73: 4903, 2001.

[154] D. Chen, X. Shao, B. Hu, and Q. Su. A Background and noise elimination method for quantitative calibration of near infrared spectra. Analytica Chimica Acta, 511:37, 2001.

[155] C. Ma and X. Shao. Continuous Wavelet Transform Applied to Removing the Fluctuating Background in Near-Infrared Spectra. Journal of Chemical Information and Computer Sciences, 44:907, 2004.

[156] L. Shao and P. R. Griffiths. Automatic Baseline Correction by Wavelet Transform for Quantitative Open-Path Fourier Transform Infrared Spectroscopy. Environmental Science and Technology, 41:7054, 2007.

[157] H. Tan and S. D. Brown. Wavelet Analysis applied to removing nonconstant varying spectroscopic background in multivariate calibration. Journal of Chemometrics, 16:228, 2002.

[158] E. L. Kosarevf and E. Pantos. Optimal smoothing of noisy data by fast Fourier transform. Journal of Physics E: Scientific Instruments, 16:537, 1983.

[159] http://www.originlab.com/.

[160] P. D. Lacharmoise, E. C. Le Ru, and P. G. Etchegoin. Guiding Molecules with Electrostatic Forces in Surface Enhanced Raman Spectroscopy. ACS Nano, 3(1):66, 2008.

[161] E. C. Le Ru, E. Blackie, M. Meyer, and P. G. Etchegoin. Surface Enhanced Raman Scattering Enhancement Factors: A Comprehensive Study. The Journal of Physical Chemistry C, 111:13794, 2007.

[162] K. Kneipp, Y. Wang, H. Kneipp, L. T. Perelman, I. Itzkan, R. R. Dasari, and M. S. Feld. Single Molecule Detection Using Surface-Enhanced Raman Scattering (SERS). Physical Reviews Letters, 78(9):1667, 1997. 
[163] P. G. Etchegoin and E. C. Le Ru. A perspective on single molecule SERS: current status and future challenges. Physical Chemistry Chemical Physics, 10:6079, 2008.

[164] P. J. G. Goulet and R. F. Aroca. Distinguishing Individual Vibrational Fingerprints: Single-Molecule Surface-Enhanced Resonance Raman Scattering from One-to-One Binary Mixtures in Langmuir-Blodgett Monolayers. Analytical Chemisty, 79:2728, 2007.

[165] P. G. Etchegoin, M. Meyer, E. Blackie, and E. C. Le Ru. Statistics of single molecule SERS signals: Fluctuation analysis with multiple analyte techniques. Analytical Chemistry, 79:8411, 2007.

[166] E. Blackie, E. C. Le Ru, M. Meyer, M. Timmer, B. Burkett, P. Northcote, and P. G. Etchegoin. Bi-analyte SERS with isotopically edited dyes. Physical Chemistry Chemical Physics, 10:4147, 2008.

[167] J. A. Dieringer, R. B. Lettan II, K. A. Scheidt, and R. P. Van Duyne. A Frequency Domain Existence Proof of Single-Molecule Surface-Enhanced Raman Spectroscopy. Journal of the American Chemical Society, 129(51): $16249,2007$.

[168] C. M. Galloway, P. G. Etchegoin, and E. C. Le Ru. Ultrafast Nonradiative Decay Rates on Metallic Surfaces by Comparing Surface-Enhanced Raman and Fluorescence Signals of Single Molecules. Physical Review Letters, 103 (6):063003, 2009.

[169] P. G. Etchegoin, P. D. Lacharmoise, and E. C. Le Ru. Influence of Photostability on Single-Molecule Surface Enhanced Raman Scattering Enhancement Factors. Analytical Chemistry, 81:682, 2009.

[170] S. Shim, C. M. Stuart, and R. A. Mathies. Resonance Raman CrossSections and Vibronic Analysis of Rhodamine 6G from Broadband Stimulated Raman Spectroscopy. ChemPhysChem, 9:697, 2008.

[171] P. Matousek, M. Towrie, and A. W. Parker. Fluorescence background suppression in Raman spectroscopy using combined Kerr gated and shifted excitation Raman difference techniques. Journal of Raman Spectroscopy, $33: 238,2002$. 
[172] M. S. Baptista and G. L. Indig. Effect of BSA Binding on Photophysical and Photochemical Properties of Triarylmethane Dyes. The Journal of Physical Chemistry B, 102(23):4678, 1998.

[173] E. C. Le Ru, M. Meyer, Blackie E., and P. G. Etchegoin. Advanced aspects of electromagnetic SERS enhancement factors at a hot spot. Journal of Raman Spectroscopy, 39:1127, 2008.

[174] M. Moskovits. Surface selection rules. J. Chem. Phys., 77(9):4408, 1982.

[175] I. A. Larkin, M. I. Stockman, M. M. Achermann, and V. I. Klimov. Dipolar emitters at nanoscale proximity of metal surfaces: Giant enhancement of relaxation in microscopic theory. Physical Review B, 69(12):121403, 2004.

[176] http://www.d3diagnostics.com/en/10452.aspx.

[177] H. W. Schroetter and H. W. Kloeckne. Raman Spectroscopy of Gases and Liquids. Springer, Berlin, 1979. 